\title{
Sexual selection, social information and male mate choice in a promiscuous fish
}

\author{
by
}

\author{
Heather L. Auld
}

A thesis submitted to the Faculty of Graduate and Postdoctoral Affairs in partial fulfillment of the requirements for the degree of

Doctor of Philosophy

in

Biology

Carleton University

Ottawa, Ontario

(C) 2016, Heather Auld 


\section{Abstract}

Compared to female mate choice, relatively little is known about male mate choice and the selective pressures that shape it. My thesis focuses on the influence of available and inexpensive social information on male mating behaviour, including mate choice. To gain insights into the influence of the social environment on male mating behaviour, I carried out seven experimental studies using a promiscuous fish, the Trinidadian guppy (Poecilia reticulata), as a model species. I used guppies from the low-predation, Upper Aripo River in Trinidad. In dichotomous choice tests, females in this population demonstrated a strong mating preference for large males and a weaker, but significant, preference for males with greater amounts of colour ornamentation. In the absence of sexual rivals, male guppies exhibited an overall preference for large females, but with consistent and repeatable inter-individual variation in both mating effort and mate choice. When conspecific males were present, male guppies paid attention to their social environment and, when given the opportunity, copied the mate choice of a nearby sexual rival. In theory, males should adjust their mating behaviour so as to minimize the potential costs of being copied. As expected, males decreased their preference for an initially preferred female in the presence of a larger, more competitive sexual rival. They also decreased their overall mating effort and used less conspicuous, but also less successful, sneak mating attempts in the presence of an audience of one or two rivals. Since the social environment can influence male mating behaviour and thus mating success, males should strategically associate with sexual rivals in a manner that will enhance their own reproductive success. Here, males preferred to socially associate with conspecific males who they had seen actively performing courtship displays towards a female compared to 
those who did not. Males who perform courtship displays may be seen as desirable social partners, as they are attractive to females and(or) may be providing social information regarding the perceived quality and(or) sexual receptivity of nearby females. Overall, my thesis highlights the important influence of the social environment on the evolution of male mating behaviour and social partner choice. 


\section{Preface}

\section{Co-authorship Statement for Chapters 3, 4, 5, 6, 7 and 8}

Of the seven data chapters in my thesis, Chapters $3,4,5,6$, and 7 have been published in peer-reviewed journals and Chapter 8 is currently under review by a peer-reviewed journal.

For Chapter 3, my supervisor, Dr. Jean-Guy Godin, and I collaboratively developed the experimental question, design, and analysis. Ryan Pusiak and I collected the data. I performed the data analysis and wrote the first draft of the manuscript. Both co-authors contributed helpful comments and editorial suggestions, which greatly improved the manuscript.

For Chapter 4, Dr. Jean-Guy Godin and I collaboratively developed the experimental question, design, and analysis. We both contributed to data collection and Dr. Godin wrote the first draft of the manuscript. I contributed to the data analysis and writing of the manuscript.

For Chapters 5 and 6, Dr. Jean-Guy Godin and I collaboratively developed the experimental questions, designs, and analyses. We both contributed to data collection. I performed the data analyses and wrote the first draft of the published manuscript, which combines the results of these two chapters. Dr. Godin contributed helpful comments and editorial suggestions, which greatly improved the manuscript. 
For Chapter 7, Dr. Jean-Guy Godin, Dr. Indar Ramnarine and I collaboratively developed the experimental question, design, and analysis. Dr. Godin and I contributed to data collection, and Dr. Ramnarine provided logistic support and facilities in Trinidad. I performed the data analysis and wrote the first draft of the manuscript. Both co-authors contributed helpful comments and editorial suggestions, which greatly improved the manuscript.

For Chapter 8, Dr. Jean-Guy Godin and I collaboratively developed the experimental question, design, and analysis. Sarah Jeswiet and I collected the data. I performed the data analysis and wrote the first draft of the manuscript. Both co-authors contributed helpful comments and editorial suggestions, which greatly improved the manuscript.

I have received permission from all co-authors to include our collaborative journal papers and submitted manuscript in my doctoral thesis. Moreover, I have permission from each journal publisher to reproduce these published papers in my thesis.

To cite this thesis as a whole document or any of the unpublished chapters I recommend the following citation:

Auld, H.L. 2016. Sexual selection, social information and male mating behaviour in a promiscuous fish. Ph.D. Thesis, Carleton University, Ottawa, Ontario, Canada 
The following citations are the published manuscripts reproduced herein and the submitted manuscript currently under peer review at the time of thesis submission:

\section{Chapter 3:}

Auld, H.L., Pusiak, R.J.P. and Godin, J.-G.J. 2016. Independent mating preferences for male body size and coloration in female Trinidadian guppies. Ethology, 122: 597 - 608.

\section{Chapter 4:}

Godin, J.-G.J. and Auld, H.L. 2013. Covariation and repeatability of male mating effort and mating preferences in a promiscuous fish. Ecology and Evolution, 3: 2020 - 2029.

\section{Chapter 5:}

Auld, H.L. and Godin, J.-G.J. 2015. Sexual voyeurs and copiers: social copying and the audience effect on male mate choice in the guppy. Behavioral Ecology and Sociobiology, 69: $1795-1807$.

\section{Chapter 6:}

Auld, H.L. and Godin, J.-G.J. 2015. Sexual voyeurs and copiers: social copying and the audience effect on male mate choice in the guppy. Behavioral Ecology and Sociobiology, 69(11): $1795-1807$. 


\section{Chapter 7:}

Auld, H.L. and Godin, J.-G.J. Male mate choice in the Trinidadian guppy is influenced by the phenotype of sexual rivals. Behavioral Ecology [provisionally accepted with minor revisions: MS\# BEHECO-2016-0299]

\section{Chapter 8:}

Auld, H.L., Jeswiet, S.B. and Godin, J.-G.J. 2015. Do male Trinidadian guppies adjust their alternative mating tactics in the presence of a rival male audience? Behavioral Ecology and Sociobiology, 69: 1191 - 1199. 


\section{Acknowledgments}

This thesis would not be possible without the generous support and guidance from my doctoral research supervisor Prof. Jean-Guy Godin. I thank him for his numerous contributions to both my thesis and my development as a scientist. The research described in my thesis was funded by Discovery Grants from the Natural Sciences and Engineering Research Council of Canada awarded to my supervisor, Jean-Guy Godin.

Thank you to Carleton University for the support they have provided during my studies here. I would like to specifically thank the staff and faculty in the Department of Biology for their assistance, encouragement and support over the past years. Thank you to Prof. Sue Bertram and Prof. Howard Rundle for serving on my thesis committee. I would also like to thank the editors, especially Prof. Andrea Pilastro, and anonymous reviewers who provided helpful comments on manuscripts published from this thesis.

Thank you to Kharran Deonaransingh, Prof. Grant Brown, Chris Elvidge, Raj Mahajibir, Brent Daniel, and Prof. Indar Ramnarine for helping with fish collection in Trinidad. I am also thankful for the logistical support provided by Prof. Indar Ramnarine at the University of the West Indies, without which this research would not have been possible. I would like to thank the Director of Fisheries, Ministry of Agriculture, Land and Marine Resources of Trinidad and Tobago for permission to collect fish from the Upper Aripo River in Trinidad.

I am very grateful for all the assistance with fish care from my lab mates both past and present, including Stacey Lee-Jenkins, Patrick Barks, Anne-Christine Auge, Sophie Potter, Ryan Pusiak, Sarah Jeswiet, Pierre-Luc Brackenridge, Adam Densmore, Alisa Miller, Valentina Scarponi, Deepro Chowdhury, Geoffrey Mazué, and many 
undergraduate students that helped out in the lab. I would also like to thank my 'half lab mates' Richard Webster, Sarah Harrison and Amy Villarreal. An extra thank you to Ryan Pusiak for assistance with fish photograph analysis and data collection and to Sarah Jeswiet, Alisa Miller and Chris Elvidge for assistance with data collection in Trinidad. The completion of this thesis would not have been possible without the support of my parents over the years and the opportunities they provided me in childhood, which led to my love and appreciation of the natural world. I thank my sister, Robyn, and brother, Cameron, for their encouragement over the years. I also thank my good friends Jennifer Duncan, Erin Maszczakiewicz, Dave Naipaul and Caleb Lewis. Thank you to the many people who have inspired and encouraged me. 


\section{Table of Contents}

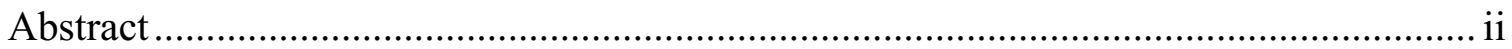

Preface

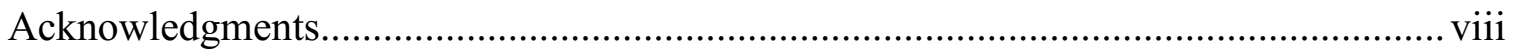

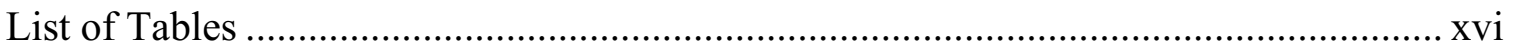

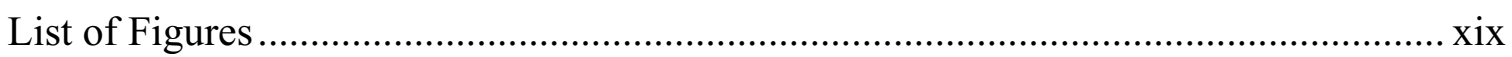

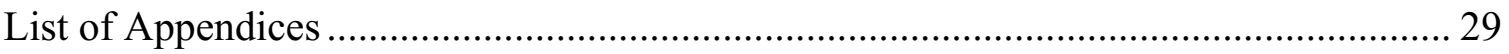

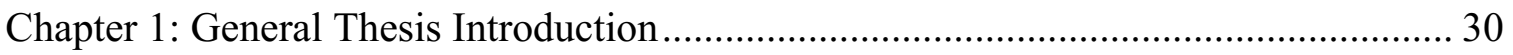

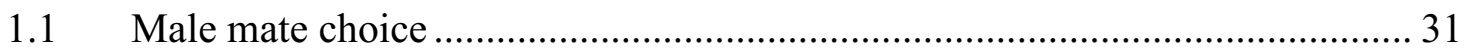

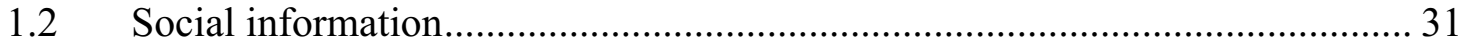

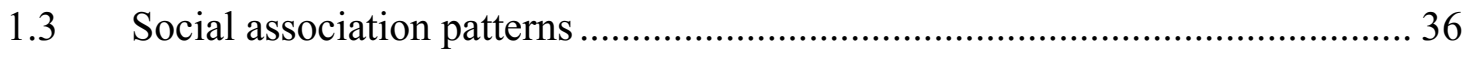

$1.4 \quad$ General objectives and organization of the thesis........................................ 37

Chapter 2: General Methods …………………………........................................... 40

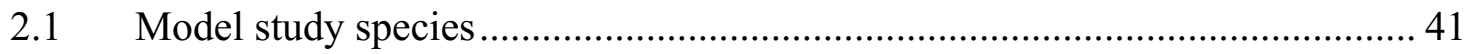

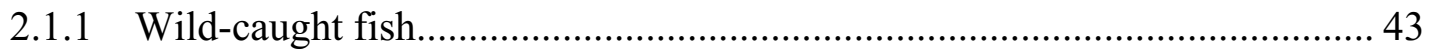

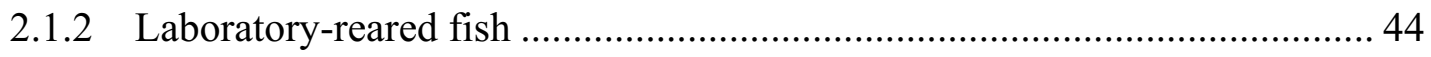

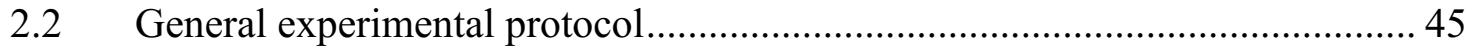

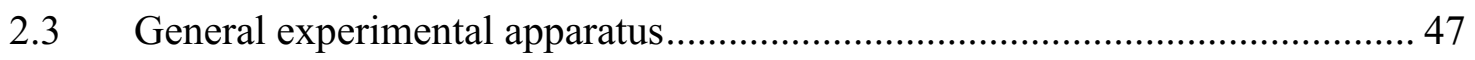

2.4 Measurement of fish body length and ornamentation....................................... 48

Chapter 3: Independent Mating Preferences for Male Body Size and Ornamentation in

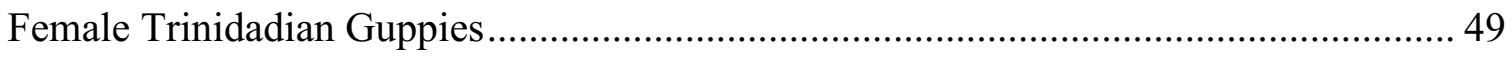




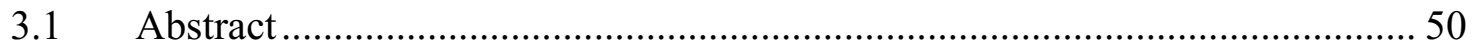

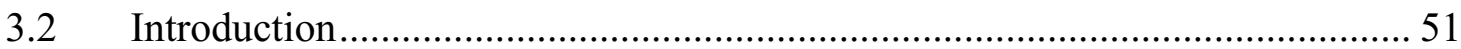

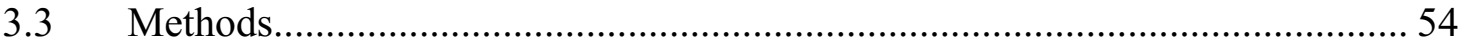

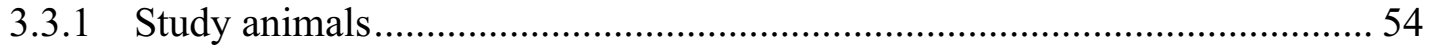

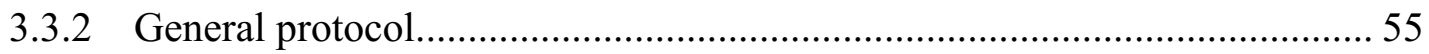

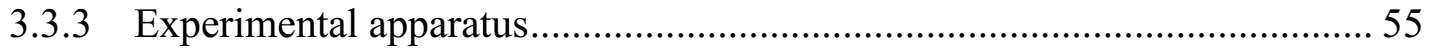

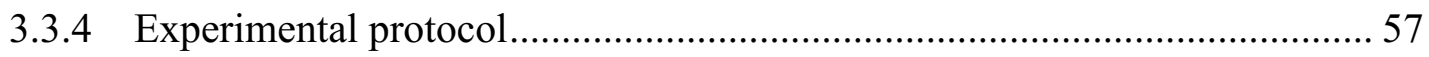

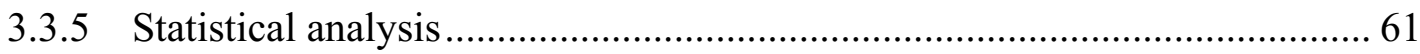

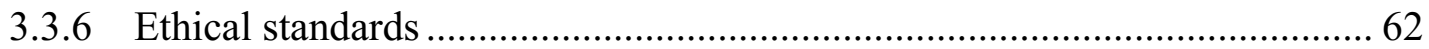

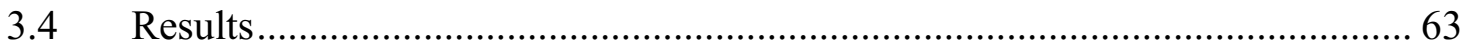

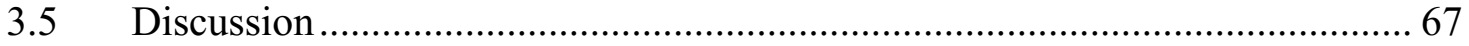

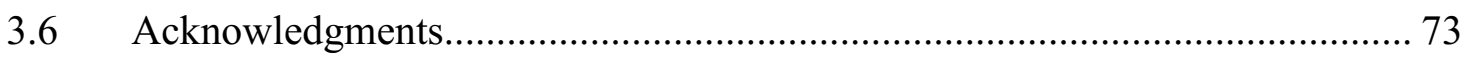

Chapter 4: Covariation and Repeatability of Male Mating Effort and Mating Preferences

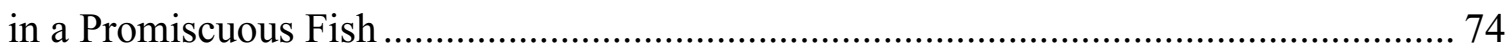

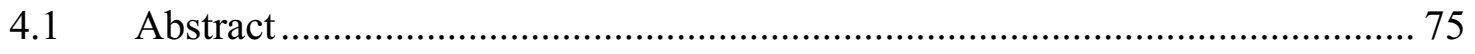

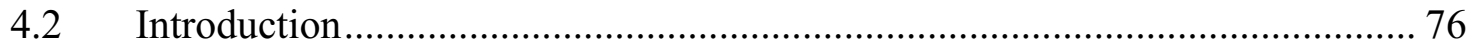

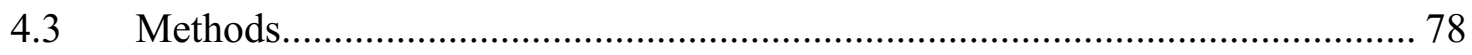

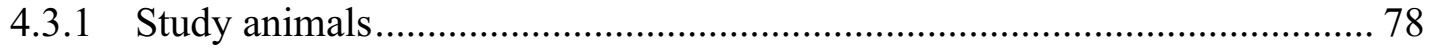

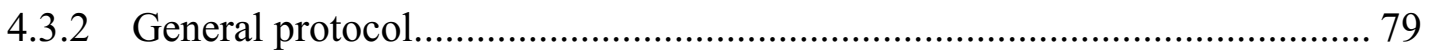

4.3.3 Experimental apparatus.......................................................................... 79

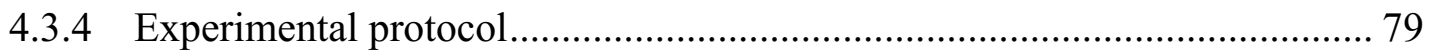

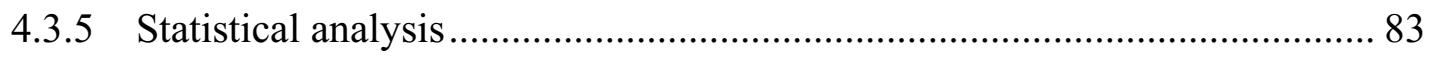

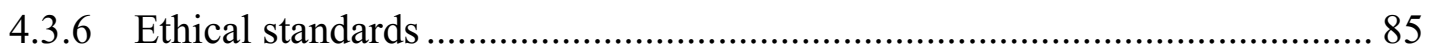




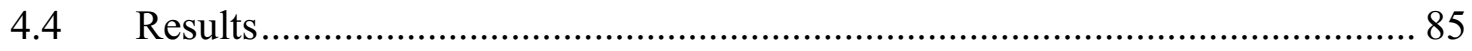

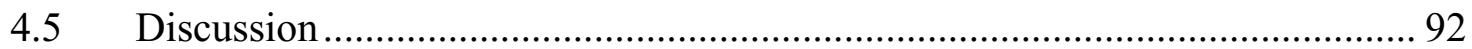

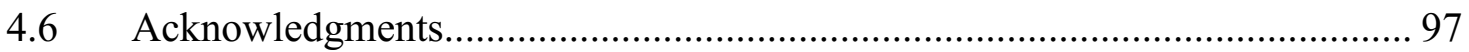

Chapter 5: Male Guppies Use Social Information and Copy the Mate Choice of Sexual

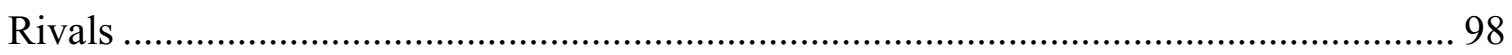

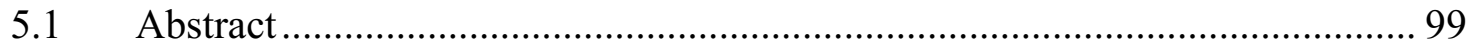

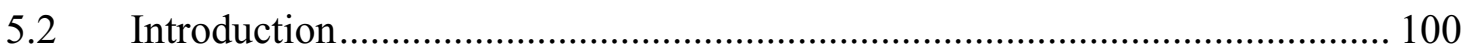

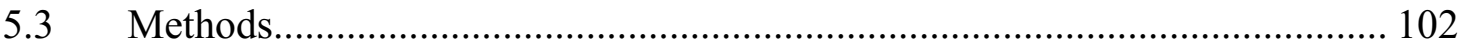

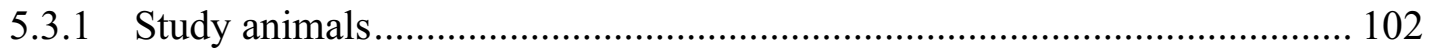

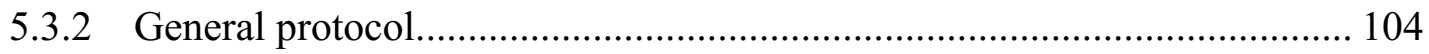

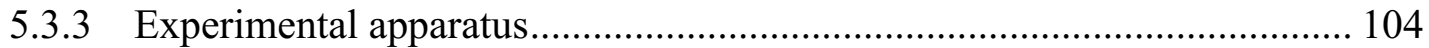

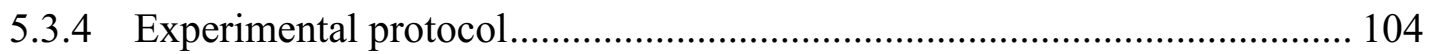

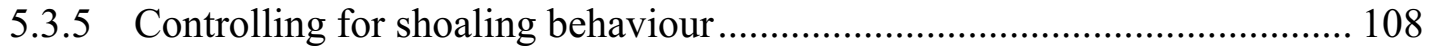

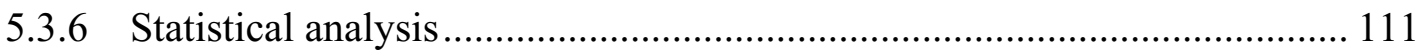

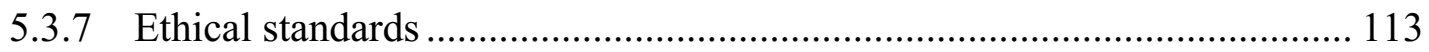

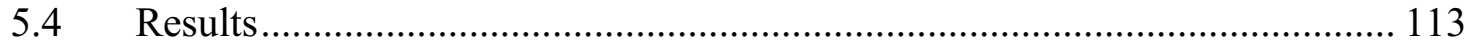

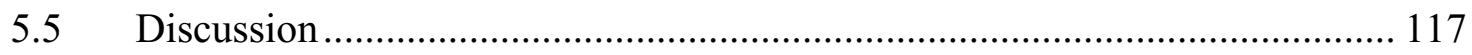

5.6 Acknowledgments....................................................................................... 120

Chapter 6: Do Male Trinidadian Guppies Adjust their Mate Choice in the Presence of a

Rival Male Audience?............................................................................................... 121

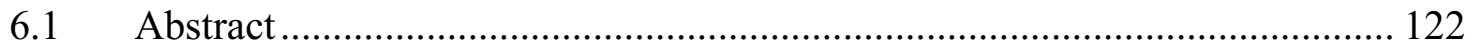

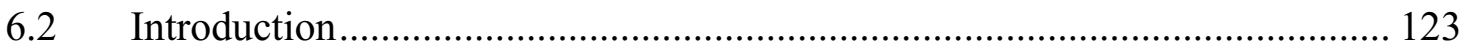

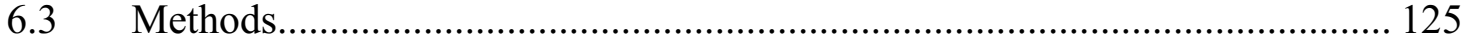




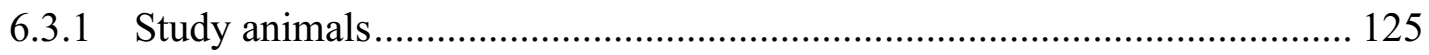

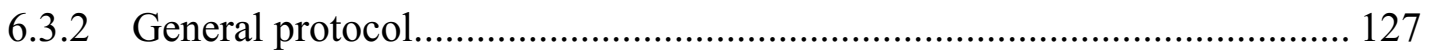

6.3.3 Experimental apparatus........................................................................... 127

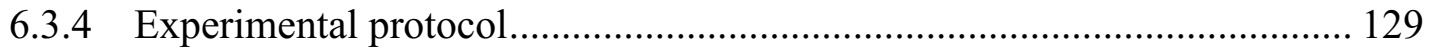

6.3.5 Control treatment for potential disturbance effect of an audience............... 131

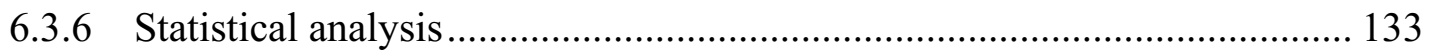

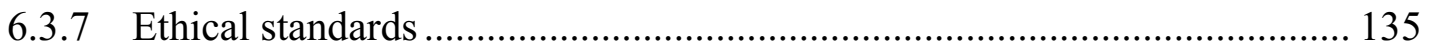

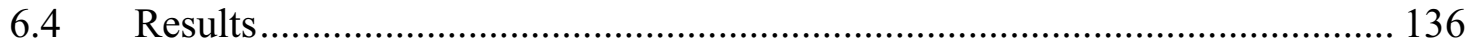

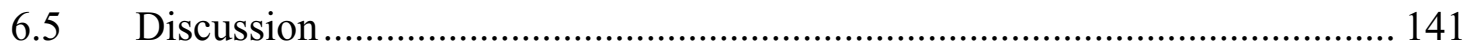

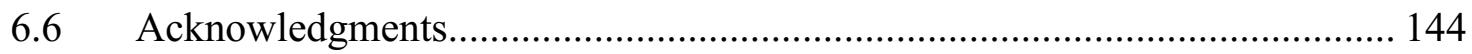

Chapter 7: Male Mate Choice is Influenced by the Phenotype of Sexual Rivals ........... 145

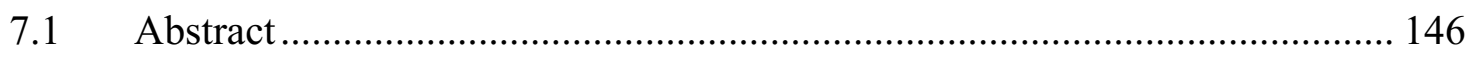

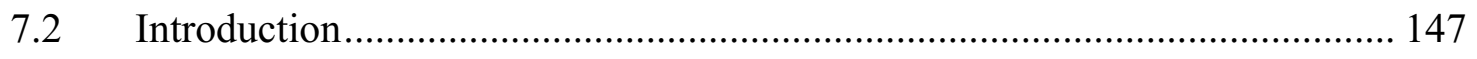

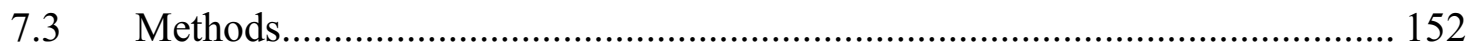

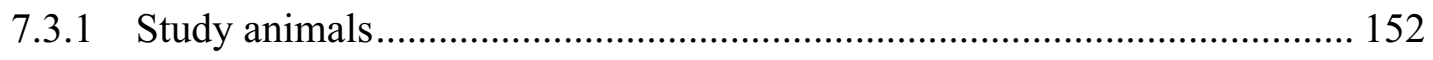

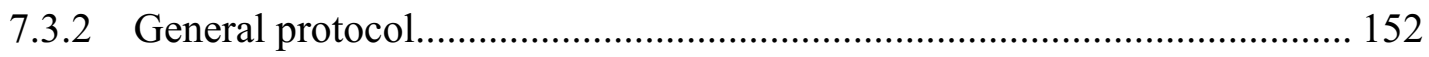

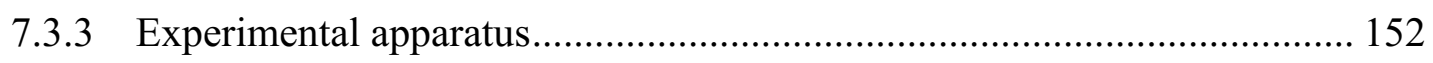

7.3.4 Experimental protocol........................................................................... 152

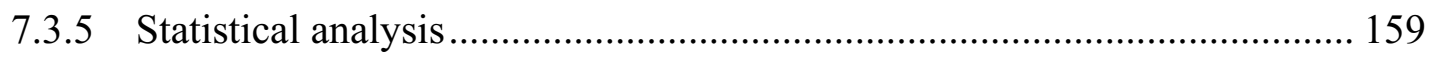

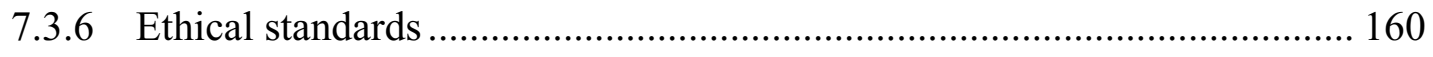

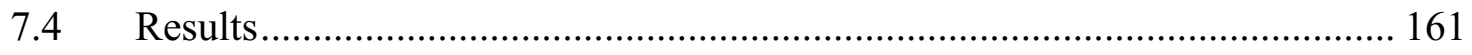

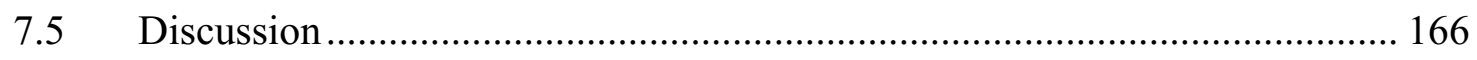

7.6 Acknowledgments.................................................................................. 172 
Chapter 8: Do Male Trinidadian Guppies Adjust their Alternative Mating Tactics in the Presence of a Rival Male Audience?

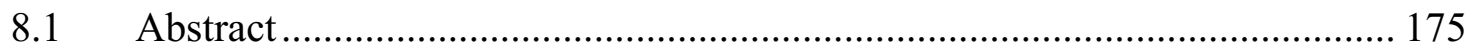

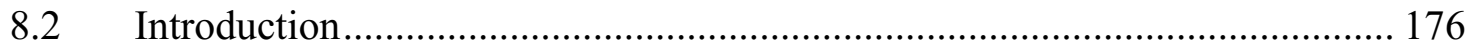

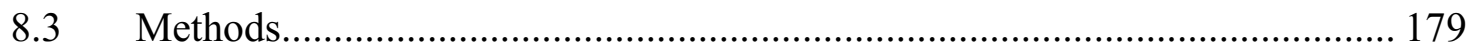

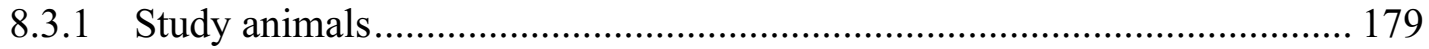

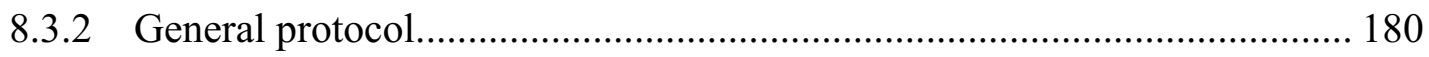

8.3.3 Experimental apparatus..................................................................... 180

8.3.4 Experimental protocol....................................................................... 180

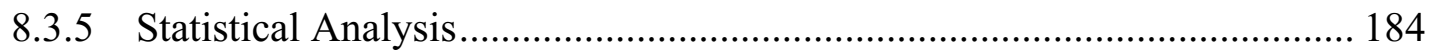

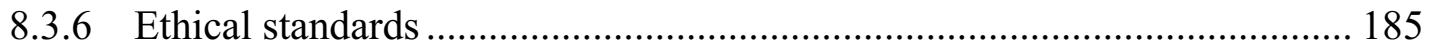

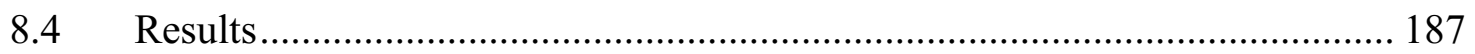

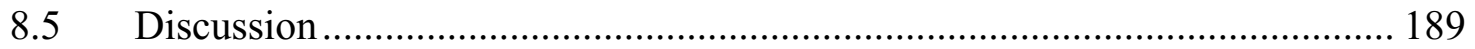

8.6 Acknowledgments.......................................................................... 194

Chapter 9: Courtship Behaviour Attracts Male Sexual Rivals in the Guppy ................ 195

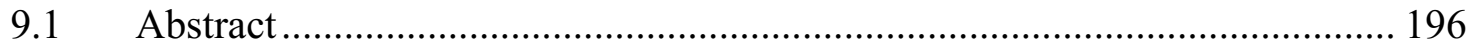

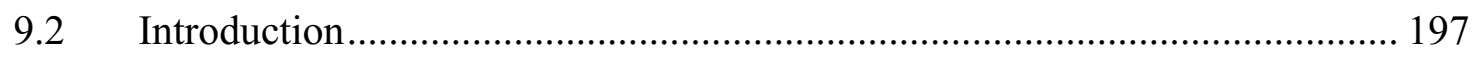

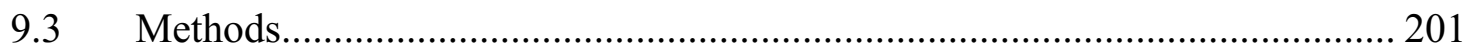

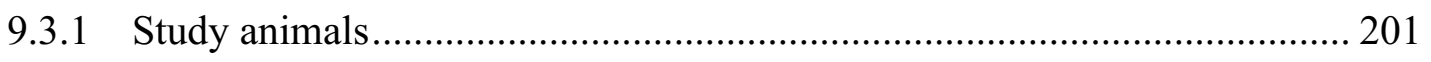

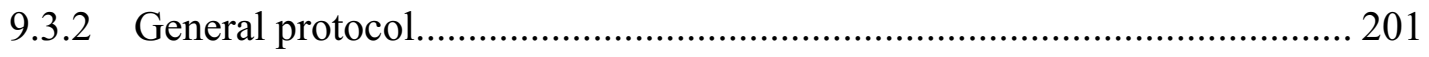

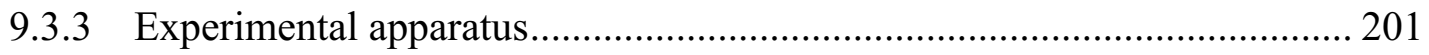

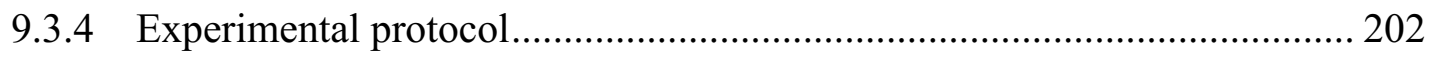

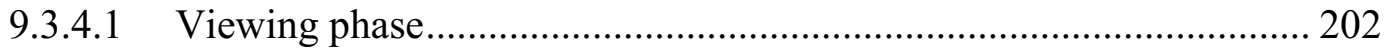


9.3.4.2 Social-partner choice phase

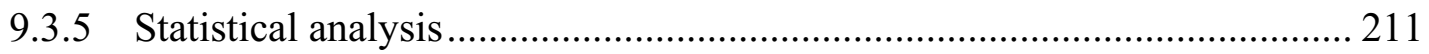

9.3.6 Ethical standards ............................................................................... 212

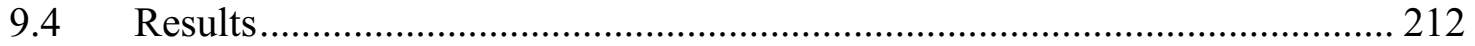

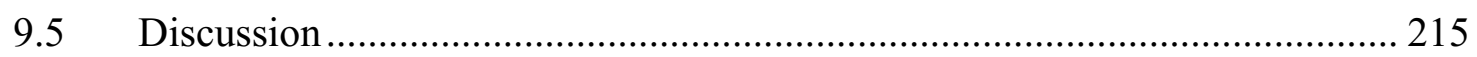

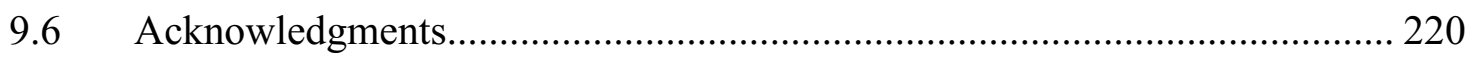

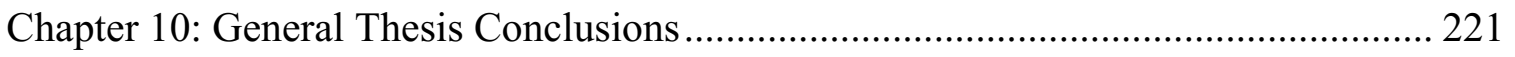

10.1 Salient findings and conclusions...................................................... 222

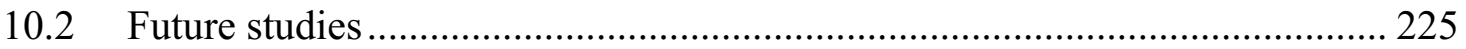

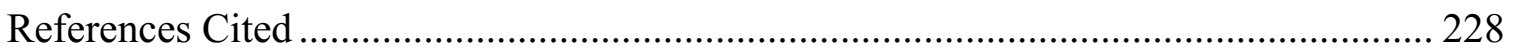

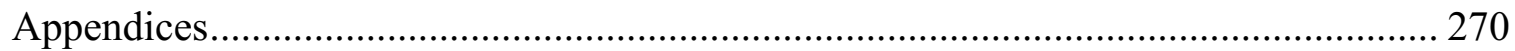




\section{List of Tables}

Table 3-1 Mean \pm SE total body length $(\mathrm{mm})$ of focal test females and total body length and body colour score (proportion of left side of body covered with black and orange colours) of the paired stimulus males categorized as relatively more attractive [(MA); either larger or more colour ornamented] and less attractive [(LA); either smaller or less colour ornamented] used in each experimental treatment. The body length and colour scores of paired stimulus males in each treatment were separately compared using the paired $t$-test. $N$ denotes the number of independent replicated trials in each treatment, and the numbers in brackets are the range. Colour scores were highly repeatable (Pearson's $r$

$=0.94, P<0.001, d f=148)$.

Table 4-1 Mean \pm SE (range) standard body lengths of the focal males and paired stimulus females used on repeated consecutive days (paired trials 1 and 2), and the mean \pm SE absolute and relative differences in the standard body length of the paired stimulus females used in individual behavioural trial. Individual focal males were tested repeatedly in paired trials 1 and 2 81

Table 5-1 Mean \pm SE (range) standard body length $(\mathrm{mm})$ and body ornamentation score (\% of male body covered by orange and black pigmentation) of guppies used in the two treatments of the mate-choice copying experiment. Results of ANOVA tests ( $F$-values) and $t$-tests for homogeneity of body length and colour score among male types, and results of paired $t$-tests comparing the body length of paired stimulus females within and between treatments, are shown. 
Table 5-2 Mean \pm SE (range) standard body length $(\mathrm{mm})$ of male guppies used in the shoaling control treatment of the mate-choice copying experiment. The result of an ANOVA test for homogeneity of body length among male types is shown.

Table 6-1 Mean \pm SE (range) standard body length $(\mathrm{mm})$ and body ornamentation score ( $\%$ of male body covered by orange and black pigmentation) of guppies used in the three experimental treatments of the audience-effect experiment. Results of the ANOVA ( $F$ values), Kruskal-Wallis ( $\chi$-values), and $t$-tests for homogeneity of body length and colour score among male types, and results of $t$-tests comparing the body length of paired stimulus females between and within treatments, are shown. The Kruskal-Wallis test was used when residuals were not normally distributed................................................... 126

Table 6-2 Mean $\pm \mathrm{SE}$ (range) standard body length ( $\mathrm{mm}$ ) of focal male guppies, audience zebrafish and paired stimulus female guppies used in the disturbance-effect control treatment. Results of paired $t$-tests comparing the body lengths of the focal male and audience zebrafish and the paired stimulus females, respectively, are shown 132

Table 7-1 Mean \pm SE (range) standard body length $(\mathrm{mm})$ and body ornamentation score (\% of male body covered by orange and black) of guppies used in each treatment separately. Results of paired $t$-tests for homogeneity of body length and colour score among male types, and results of paired $t$-tests comparing the body length of paired stimulus females within and between treatments, are shown. 
Table 8-1 Mean $\pm \mathrm{SE}$ (range) standard body length $(\mathrm{mm})$ and body ornamentation score ( $\%$ of male body covered by orange pigmentation) of guppies used in the three audience treatments. Results of ANOVA tests ( $F$-values), Kruskal-Wallis ( $\chi$-values) and $t$-tests for homogeneity of body length and colour score between focal and audience males are indicated. Kruskal-Wallis tests were used when residuals did not meet assumptions of normality. 186

Table 9-1 Mean \pm SE (range) standard body length $(\mathrm{mm})$ and body ornamentation score (proportion of male body covered by orange and black pigmentation) of guppies used in the three treatments. Results of the ANOVA ( $F$-values), Kruskal-Wallis ( $\chi$-values), and $t$ tests for homogeneity of body length and colour score among male types, and results of $t$ tests comparing the body length of stimulus females between treatments, are shown. The Kruskal-Wallis test was used when residuals were not normally distributed. 210 


\section{List of Figures}

Figure 3-1 Schematic top view of the experimental apparatus (not to scale). The test aquarium ( $40 \times 20 \times 25 \mathrm{~cm} ; \mathrm{L} \times \mathrm{W} \times \mathrm{H})$ held the focal female and the end compartments (6 $\times 20 \times 22 \mathrm{~cm}$ held the stimulus males (SM1 and SM2)). The end compartments held one stimulus male each. One stimulus male was either larger or more colour ornamented than the other stimulus male. The stippled circle represents a removable clear Plexiglas cylinder ( $7 \mathrm{~cm}$ diam.), in which the focal female could temporarily be placed. The dashed lines demarcate $10-\mathrm{cm}$ mating preference zones.

Figure 3-2 Mean \pm SE female preference (= proportion of total association time spent near) for $(a)$ the larger or more colour ornamented male in Treatments 1 and 2, respectively, and (b) the more colour ornamented male in Treatment 2 with a greater proportion of his body covered with orange and black pigmentation, separately. The horizontal stippled lines at proportion 0.5 denote no choice (i.e. chance level)

Figure 3-3 The proportion of time focal females spent in the preference zone of the (a) larger or (b) more colour ornamented of the paired stimulus males as a function of female body length. In Treatment 2 (panel $b$ ), male ornamentation is the proportion of their body covered in orange and black pigmentation. Non-transformed data and linear regression lines are shown.

Figure 4-1 Mating effort and mating preference of focal males for the larger female in each of the repeated trials. Mating effort is depicted as the mean $\pm \mathrm{SE}$ percent of total 
sexual acts directed by focal males towards the larger female, and mating preference as the mean $\pm \mathrm{SE}$ percent time focal males spent associating with the larger female. The horizontal line denotes random choice between the paired stimulus females. The asterisk above individual histogram bars indicates a significant $(P<0.0001$; Wilcoxon signedrank test) difference between the observed mean score and that expected from chance (i.e. 50\%) for that particular trial. The $P$-value above each pair of histogram bars (paired trials 1 and 2) was obtained using LMM models testing for any effect of trial number on the behavioural scores of males in each experiment.

Figure 4-2 Male mating effort expressed as the mean \pm SE frequency (number/40 min) of each of the different component sexual acts, and their total sum, directed by focal males towards each of the paired stimulus females over the course of the repeated paired trials. $\mathrm{AP}=$ approach, $\mathrm{SD}=$ courtship sigmoid display, $\mathrm{GN}=$ gonopodial $\operatorname{nip}, \mathrm{CA}=$ copulation attempt. $* * * P<0.0001$, obtained using the Wilcoxon signed-ranks test.... 88

Figure 4-3 Relationships between the mating preference of focal males, based on percent association time with the larger female, and their mating effort (panels A and B) and courtship effort (panels C and D) for paired repeated trials 1 and 2. The best-fit lines were obtained using simple linear regression analysis. The correlation coefficient $\left(r_{s}\right)$ and $P$ values shown were obtained using the Spearman rank correlation analysis. 90

Figure 4-4 Relationships between the mating effort scores of focal males, based on the percent of sexual acts directed towards the larger female (panel A), and their mating 
preference scores, based on percent association time with the larger female (panel B), during trial 1 and their scores one day later in repeated trial 2. The best-fit lines, and associated $r^{2}$ and $P$ values, were obtained using simple linear regression analysis. Repeatability $(R)$ estimates were obtained using the linear mixed-effects model with the restricted maximum-likelihood (REML) method described in Nakagawa and Schielzeth (2010)

Figure 5-1 Schematic top view of the experimental apparatus used in the mate-choice copying experiment. The central compartment $(40 \times 20 \times 25 \mathrm{~cm} ; \mathrm{L} \times \mathrm{W} \times \mathrm{H})$ held the focal male (F) and the end compartments $(15 \times 20 \times 22 \mathrm{~cm})$ held the model males (M) and (or) stimulus females, depending on the treatment. The circle represents a removable clear Plexiglas cylinder ( $7 \mathrm{~cm}$ diam.), in which the focal male could temporarily be placed. The thick dark line in front of one of the end compartments denotes an opaque Plexiglas screen. Dotted lines represent removable clear Plexiglas partitions that allowed the end compartments to be either divided in half or left open. 105

Figure 5-2 Mating preferences of focal males for the female that was initially not preferred in Preference Test 1 after they had observed that same female in either the absence (Control treatment; no copying opportunity) or the presence of a conspecific male model behind a clear Plexiglas partition (Copying treatment; copying opportunity) in Preference Test 2. Mating preferences are shown using three measures: $(a)$ mean $( \pm$ SE) proportion of time that focal males spent associating with the initially non-preferred female in Preference Test 1 (open bar) and in Preference Test 2 (filled bar); (b) mean ( \pm 
SE) preference difference score between Preference Test 2 and Preference Test 1 (a positive value denotes an increase in the focal male's preference for the initially nonpreferred female in Preference Test 2); and (c) percentage of males that reversed their initial preference (observed in Preference Test 1) in Preference Test 2. Significant $P$ values are shown for paired comparisons; n.s. denotes not significant $(P>0.05) \ldots \ldots . . .114$

Figure 5-3 Comparison of the mating behaviour of focal males in Preference Test 1 compared to their mating behaviour in Preference Test 2 in either the absence of any copying opportunity or when a copying opportunity was present. Mating behaviour is shown as $(a)$ the mean $\pm \mathrm{SE}$ amount of time that the focal males spent associating with either of the stimulus females in Preference Test 1 and Preference Test 2, (b) the mean \pm SE change in the amount of time that focal males spent associating with either stimulus female in Preference Test 2 relative to the amount of time they spent with either stimulus female in Preference Test 1 , and (c) the mean \pm SE amount of time that focal males spent associating with the initially non-preferred female (open bars) and the initially preferred female (grey bars) in Preference Test 1 and Test 2 for each treatment separately. 116

Figure 6-1 Schematic top view of the experimental apparatus. The central compartment held the focal male (F) and the 'audience' fish (constrained in a clear cylinder) and the end compartments held the stimulus females. In panel (b), each circle represents a removable clear Plexiglas cylinder in Preference Test 2; one cylinder (most central - not shown) temporarily held the focal male and the other cylinder was either empty or held 
the 'audience' fish, depending on the treatment. The dashed lines in the test aquarium demarcate the $10-\mathrm{cm}$ preference zones

Figure 6-2 Comparison of the mating preferences of focal males for the particular female initially preferred in Preference Test 1 compared to their preferences for the same female in the paired Preference Test 2 when not being observed by an audience conspecific male (no audience) or being observed by either an audience one conspecific male, two conspecific males, or one heterospecific zebrafish (control for a general disturbance effect). The vertical dashed line separates the results obtained from the three treatments of Experiment 2 from the control (zebrafish audience) treatment. The mating preferences of focal males are shown using three measures: $(a)$ mean $( \pm \mathrm{SE})$ proportion of time that focal males spent associating with the initially-preferred female in Preference Test 1 (open bar) and in Preference Test 2 (filled bar); (b) mean ( \pm SE) preference difference score between Preference Test 2 and Preference Test 1 (a negative value denotes a decrease in the focal male's preference for the initially-preferred female in Preference Test 2); and (c) the percentage of males that reversed their initial preference (observed in Preference Test 1$)$ in Preference Test 2 . Only significant comparisons $(P<0.05)$ are shown in panel (a). In the other two panels, histogram bars with dissimilar letters are significantly different $(P<0.05)$ from each other. The multiple paired comparisons in panels $\mathrm{b}$ and c were carried out using paired $t$-tests and the $G$-test, respectively, with the FDR for multiple comparisons controlled for 138 
Figure 6-3 Comparison of the mating behaviour of focal males in Preference Test 1 compared to their mating behaviour in Preference Test 2 when either not being observed by an audience conspecific male (no male; Treatment 1) or being observed by either an audience one conspecific male (one male; Treatment 2), two conspecific males (two males; Treatment 3), or one heterospecific zebrafish (control for a general disturbance effect). The vertical dashed line separates the results obtained from the three guppy male audience treatments from the control (zebrafish audience) treatment. The mating behaviour of focal males is shown as $(a)$ the mean $\pm \mathrm{SE}$ amount of time that the focal males spent associating with either of the stimulus females in Preference Test 1 and Preference Test 2, $(b)$ the mean $\pm \mathrm{SE}$ change in the amount of time that focal males spent associating with either stimulus female in Preference Test 2 relative to the amount of time he spent with either stimulus female in Preference Test 1, and (c) the mean \pm SE amount of time that focal males spent associating with the initially non-preferred female (open bars) and the initially preferred female (grey bars) in Preference Test 1 and 2 for each treatment separately. Histogram bars with dissimilar letters are significantly different $(P$ $<0.05)$ from each other. The multiple paired comparisons in panel $(a)$ were carried out using the Tukey Test. 140

Figure 7-1 Schematic top view of the experimental apparatuses used. The central compartment (40 x $20 \times 25 \mathrm{~cm}$; L x W x H) held the focal male (F) and the audience male (depending on the preference test) and the end compartments $(6 \times 20 \times 22 \mathrm{~cm})$ held the stimulus females. In panel $(b)$, the central compartment concurrently held the free- 
swimming focal male and the 'audience' male (constrained in a clear Plexiglas cylinder, $7 \mathrm{~cm}$ diam.) during Preference Test 2

Figure 7-2 Comparison of the initial mating preference of focal males for one of the two stimulus females presented in the absence of an audience male (Preference Test 1) compared to their preference for the same female in the presence of an eavesdropping audience male (Preference Test 2), who was either smaller and less colour ornamented (Treatment 1), smaller and more ornamented (Treatment 2), larger and less ornamented (Treatment 3) or larger and more ornamented (Treatment 4) than the focal male. The mating preferences of focal males are shown using two measures: $(a)$ mean $\pm \mathrm{SE}$ proportion of time that focal males spent associating with the initially-preferred female in Preference Test 1 (open bar) and Preference Test 2 (filled bar); (b) mean ( \pm SE) preference difference score between Preference Test 2 and Preference Test 1 (a negative value denotes a decrease in the focal male's preference for the initially-preferred female in Preference Test 2 calculated from linear model fitted values). Histogram bars with dissimilar letters are significantly different $(P<0.05)$ from each other 164

Figure 7-3 Relationship between the initial mating preference of focal males for a particular female (in Preference Test 1) and the change in their mating preference for the initially-preferred female in the presence of an audience male (Preference Test 2) who was either smaller and less colour ornamented (Treatment 1), smaller and more ornamented (Treatment 2), larger and less ornamented (Treatment 3) or larger and more ornamented (Treatment 4) than themselves. The arcsine-transformed partial residuals 
from the linear model are plotted. In each panel, the best-fit regression line and the correlation coefficient (with confidence intervals) of the relationship are shown.

Figure 8-1 Schematic top view of the experimental apparatus. In Test 1, the test aquarium ( $40 \times 20 \times 25 \mathrm{~cm} ; \mathrm{L} \times \mathrm{W} \times \mathrm{H}$ ) held the focal male and stimulus female. The circle represents a removable clear Plexiglas cylinder (7 cm diam.), in which the focal male could temporarily be placed. In Test 2 , the aquarium held the focal male, the stimulus female and the 'audience' fish (constrained in a separate clear cylinder (not shown) in the centre of the aquarium throughout the test). The audience cylinder was either empty or held one or two conspecific audience males, depending on the treatment.

Figure 8-2 Comparison of the mating effort of focal males between two paired 20-min tests across three audience treatments: (i) not being observed by a conspecific audience male ( 0 audience male present; Treatment 1$)$, or (ii) being observed by either an audience of one conspecific male (Treatment 2) or (iii) two conspecific males (Treatment 3). The mating behaviour of focal males is shown as the mean $( \pm \mathrm{SE})$ change in the frequencies of (a) courtship displays and (b) sneak copulation attempts, and (c) the proportion of total mating attempts that are courtship displays between the paired tests. Only statistically significant paired comparisons across treatments (using permutational MANOVAs) are shown. 188 
Figure 9 -1 Schematic top view of the experimental apparatus used in the viewing phase of the experiment. The larger viewing aquarium $(40 \times 20 \times 25 \mathrm{~cm}$; L x W x H), which held the focal male, and the five smaller compartments $(6 \times 20 \times 25 \mathrm{~cm})$, which held the stimulus fish, were placed in a warm water bath $(100 \times 100 \times 30 \mathrm{~cm})$ as shown. The dashed lines represent 10 -cm viewing zones directly in line with the end compartments containing the stimulus males, and the thick solid lines represent removable opaque Plexiglas screens. The central small compartment held a stimulus female or male

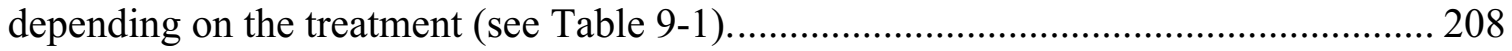

Figure 9-2 Schematic top view of the experimental apparatus used in the partner-choice phase of the experiment. The central compartment $(40 \times 20 \times 25 \mathrm{~cm} ; \mathrm{L} \times \mathrm{W} \times \mathrm{H})$ held the focal male and the end compartments $(6 \times 20 \times 22 \mathrm{~cm})$ held the 'winner' and 'loser' demonstrator stimulus males. The dashed lines represent 10-cm (social association) preference zones, and the thick solid lines represent removable opaque Plexiglas screens. The dotted circle denotes the removable clear Plexiglas cylinder $(7 \mathrm{~cm} \mathrm{diam}$.) that temporarily held the focal male. 209

Figure 9-3 Mean \pm SE proportion of total association time the focal male spent near the winner stimulus male in the three treatments during (a) the viewing phase and (b) the social partner-choice phase of the experiment. The horizontal stippled lines at proportion 0.5 denote no choice (i.e. chance level). Histogram bars with dissimilar letters are significantly different $(P<0.05)$ from each other. In the male control treatment, the stimulus female in the central compartment of the viewing phase was replaced by a 
stimulus male. The number of replicate trials (with no side bias) for each treatment is shown inside inside the histogram bars. 


\section{List of Appendices}

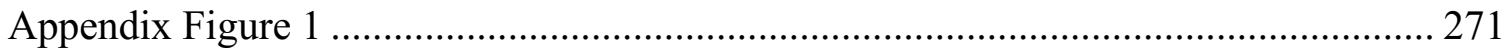

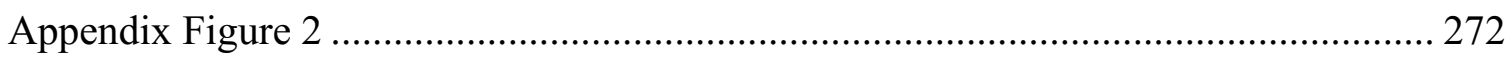

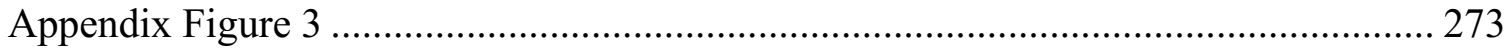




\section{Chapter 1:}

\section{General Thesis Introduction}




\subsection{Male mate choice}

Males have traditionally been regarded as being indiscriminate when mating with females (Clutton-Brock and Vincent 1991; Andersson 1994; Clutton-Brock 2007; Edward and Chapman 2011). However, accumulating evidence suggests that mate choice by males is relatively common in nature (reviewed in Amundsen 2000; Bonduriansky 2001; CluttonBrock 2007; Edward and Chapman 2011). Despite recent advances (reviewed in Amundsen 2000; Bonduriansky 2001; Clutton-Brock 2007; Edward and Chapman 2011), our understanding of the evolution and maintenance of male mate choice and its importance in driving evolutionary change remains limited compared with that for female mate choice. Theoretically, male mate choice can evolve under a wide range of conditions (Bonduriansky 2001; Wedell et al. 2002; Servedio and Lande 2006; CluttonBrock 2007; Edward and Chapman 2011; South et al. 2012). Conditions favouring its evolution include costly and/or limited sperm production, sperm competition among males, female preference for males exhibiting high courtship effort, and variation in female quality, male mating effort and costs of mate choice. For male mate choice to evolve, choosy males must accrue benefits that offset the costs of choice (Parker 1983; Edward and Chapman 2011). Therefore, male preference for a female trait is only likely to evolve when that trait confers a reproductive advantage, such as greater fecundity. Male preference for arbitrary female traits is unlikely to evolve.

\subsection{Social information}

In complex and dynamic environments, such as social environments, genetically transmitted information, such as heritable mate choice preferences, alone are often not 
sufficient to ensure optimal behavioural decisions resulting in maximum fitness (Danchin et al. 2004). Animals can supplement genetic information by obtaining external information about environmental parameters either directly via trial and error tactics or via information produced by others. Trial and error sampling can be a more accurate source of information, but may also be more time consuming, risky and more energetically costly than acquiring information through observing the behaviours and(or) decisions of others (Danchin et al. 2004). In addition, social animals can also obtain information from observing and(or) communicating with others (Danchin et al. 2004). There are two main ways information can move through a social network. It can be transmitted from one individual to others through direct communication via signals from sender to receiver(s) or it can be obtained indirectly by observing the interactions and behavioural decisions of others. This transfer of information is not limited to two individuals and can occur anytime within the context of a social network, as long as several animals are within signalling and receiving range of each other (McGregor and Peake 2000).

There are several types of information that have been recognised based on the manner in which the information is acquired, either through direct signalling, through observation of others, or what sort of information the cue is providing. Perhaps the most well studied and obvious form of information transfer is direct communication via signals from one individual to others. Signals can be visual, acoustic, tactile, chemical and electrical in nature (Bradbury and Vehrencamp 1998).

Information obtained indirectly by an individual through observation of others that require similar resources is referred to as inadvertent social information (ISI) (Danchin et 
al. 2007). For example, ISI might reveal the location of a suitable foraging location. If that information is also indicative of the quality of an environmental parameter (e.g. food density), it is referred to as public information (PI) (Danchin et al. 2004; Valone 2007). PI can influence the behaviour of individuals in many species across a wide range of behavioural contexts (Danchin et al. 2004; Kendal et al. 2005).

It is well known that animals use ISI and PI within communication networks with respect to the abundance and quality of food, the presence of predators and quality of mates, for example (Danchin et al. 2004; McGregor 2005). Mate choice has traditionally been viewed as an interaction, and exchange of information, between just two individuals, but recent studies have highlighted the role of social context in mating behaviour (Abrahams 1993; Amundsen 2000; Johnstone 2001; Earley and Dugatkin 2005; Godin et al. 2005; Nordell and Valone 1998; Pruett-Jones 1992; Kirkpatrick and Dugatkin 1994; Westneat et al. 2000; White 2004). Investigations of the acquisition and use of social information by individual animals in the context of mating has led to the characterization of a number of social phenomena, such as social eavesdropping, mate choice copying and the audience effect. These social phenomena are described below.

Social eavesdropping occurs when a bystander gathers information on other individuals by attending to their signalling interactions with conspecifics (Peake 2005). Eavesdropping can be beneficial for the bystander as it provides a low-cost, low-risk alternative to gathering the same information through direct interactions with the individuals being eavesdropped on (Dabelsteen 2005). Alternatively, it may not always be beneficial for the individuals being eavesdropped upon to have others extract information about the outcome of their interaction (Dabelsteen 2005). For example, 
female fighting fish, Betta splendens, that have witnessed a male-male aggressive interaction will bias their mate choice toward the winner of the interaction, whereas a female that did not witness the interaction will show no bias towards either the winner or the loser (Herb et al. 2003). In this case, it would be disadvantageous for the loser of the interaction to have that information made public, as it would decrease his chances of obtaining a mating.

Copying involves repeating the behaviour the observer has seen others exhibit. Individuals can copy a foraging patch, a nesting location or the mate of another individual. Mate choice copying is a phenomenon in which one sex, usually female, biases mating toward individuals that they have previously observed in successful sexual encounters with a conspecific (Dugatkin 1992; Galef and White 1998; Witte et al. 2015). Consequently, once an individual obtains one mating it can significantly increase the probability of that individual receiving more matings compared to one who did not. Mate choice copying has been studied most commonly in highly social species, such as lekking birds (e.g. Galef and White 1998) and polygynous fishes (e.g. Dugatkin et al. 2002; Godin et al. 2005; Alonzo 2008; Godin and Hair 2009), and in females due to its potential effects on sexual selection (e.g. Wade and Pruett-Jones 1990; Kirkpatrick and Dugatkin 1994; Alonzo 2008). To date, male mate choice copying has remained largely unexplored. The few studies that have investigated male mate choice copying have focused on poeciliid fishes (Schlupp and Ryan 1994; Schlupp and Ryan 1997; Witte and Ryan 2002), with the exception of the sex-role reversed deep-snouted pipefish, Syngnathus typhle (Widemo 2005). Most studies of the effects of social factors on mating behaviour have focused on either 'social eavesdropping' or 'mate choice copying' in 
females, with both phenomena being documented across a wide range of taxa including insects (e.g. Mery et al. 2009, but see Auld et al. 2009), birds (e.g. Mennill et al. 2002; Naguib et al. 2004; White and Galef 1999), and fishes (e.g. Dugatkin 1992; Godin et al. 2005; Godin and Hair 2009). Because most of this research focuses on female choice, we still have a very limited understanding of the potential roles that both these social phenomena may play in the mating decisions of males.

Another related social network phenomenon is the potential effect of a noninteractive bystander on the behaviour of the main signaller. This phenomenon is referred to as the 'audience effect' and is defined as changes in the behaviour of the signaller during an interaction between individuals caused by the mere presence of an audience nearby (Matos and Schlupp 2005). An audience is defined as one or more individuals that affect the behaviour of the signaller when they are present and detected but do not participate in the interaction (Matos and Schlupp 2005). The audience effect is specific to communication networks as, by definition, it can only occur in situations involving a minimum of three individuals: two individuals engaged in a signalling event and one individual making up the audience (Matos and Schlupp 2005). Recently, studies have described this effect in several vertebrate species, including fishes (Matos and McGregor 2002; Dzieweczynski and Rowland 2004; Dzieweczynski et al. 2005, 2006, 2009; Hibler and Houde 2006; Plath et al. 2008a, b, c; Plath and Schlupp 2008; Padur et al. 2009; Plath et al. 2009; Ziege et al. 2008, 2009), mammals (Pollick et al. 2005; Le Roux et al. 2008) and birds (Evans and Marler 1994; Striedter et al. 2003). Until recently, most studies of the audience effect have focused mainly on food calling and predator warning behaviours. However, more recent work has also demonstrated evidence of an audience 
effect in a sexual context (e.g. Dzieweczynski and Rowland 2004, Wong 2004, Plath et al. 2008a, b, 2009, Ziege et al. 2008).

This growing body of evidence for the influence of social information on male mate choice may also contribute to our understanding of social association patterns among males in a population.

\subsection{Social association patterns}

The potential fitness costs and benefits associated with social phenomena such as mate choice copying and the audience effect are directly linked to the individuals involved in social interactions. It is well known that social animals often form groups or aggregations via non-random associations with others (Krause and Ruxton 2002). Social association patterns are important because they affect the social information that an individual has access to and social information can vary in quality and availability (Danchin et al. 2004;

Dall et al. 2005). Because of this, individuals may compete for access to optimal information or a position within a social network that allows them access to accurate information. For example, a given individual would likely receive greater benefits from copying the location of a food patch exploited by a healthy, well-nourished individual than the food patch of an individual that appears unhealthy, as the nutritional state or 'success' of the individual foraging at a given food patch may be assessed as inadvertent public information that indicates the quality of the patch.

Knowledge of social association patterns and communication networks contributes to our understanding of the evolution of social living and the dynamics of social groups. Although there has been growing interest in social networks in the past few years and 
social association patterns have been studied in both males and females with respect to both predator avoidance and foraging opportunities (see Krause and Ruxton 2002; Croft et al. 2008), there has been very little work done on how the sexual context may affect social association patterns among males (e.g. Oh and Badyaev 2010; Gasparini et al. 2013; Auge et al. 2016). Importantly, acquisition and use of social information by individuals within the contexts of social eavesdropping, mate choice copying and audience effects has potential implications for social-partner choice among males and thus the composition of animal social networks.

\subsection{General objectives and organization of the thesis}

While there has been considerable work done on social factors influencing female mate choice, to date there is comparatively little research on the potential acquisition and use of social information by males when making mating decisions. Research on public information transfer and its consequences for male mating behaviour can help us better understand how information travels through social networks and the underlying mechanisms of social association patterns. Although we know that social associations are commonly not random (Krause and Ruxton 2002; Croft et al. 2008), we currently know very little about the mechanisms underlying their formation and maintenance. For example, size-assortative shoaling, or group behaviour, is thought to be primarily driven by predator risk (Krause and Ruxton 2002); however, fishes in low-predation sites also exhibit size-assortative shoaling, which suggests that there are other driving forces shaping the structure and dynamics of social networks (Krause and Ruxton 2002). Identifying the potential impact and flow of information among individuals within a 
sexual context can help us further identify and understand the dynamics of communication networks within populations. Investigating how social associations are formed within a sexual context will contribute to a deeper understanding of the structure of social and communication networks in general.

My thesis research addresses the following inter-related questions using the Trinidadian guppy (Poecilia reticulata), originating from a low-predation natural population, as a model study species:

1) Do female guppies from my study population demonstrate typically strong mating preferences for males who are larger and(or) more colour ornamented?

2) In the absence of nearby conspecifics, do male guppies demonstrate consistent and repeatable mating preferences for larger and presumably more reproductively valuable females?

3) When inexpensive social information regarding the mating preference of a sexual rival is available, do audience male guppies use this information and copy the apparent mate choice of that sexual rival?

4) To avoid any sexual competition associated with having their mate choice copied, do male guppies alter their initial mate choice in the presence of a sexual rival (i.e. an audience), and does any audience effect increase in magnitude with an increasing number of audience males present?

5) Does the phenotype, and presumably the sexual attractiveness and 
competitiveness, of a nearby audience male affect the mating preference of focal males?

6) Does the presence of a nearby audience sexual rival influence the courtship and mating effort of male guppies?

7) Do male guppies non-randomly associate socially with other males based on public information gleaned from previously observing the courtship behaviour (and thus potential sexual attractiveness) of the latter males towards females?

I sequentially addressed these questions using manipulative experiments, which are described in the following chapters. 
Chapter 2:

\section{General Methods}




\subsection{Model study species}

As stated at the end of Chapter 1, I used the Trinidadian guppy as my model study species. The guppy is a live bearing, internally fertilising, freshwater fish species native to Trinidad and Tobago and certain adjacent areas in Venezuela (Houde 1997), which has long been an important model system for the study of the evolution of behaviour (Houde 1997; Magurran 2005). The species is sexually dimorphic, with adult males being on average more colour ornamented and smaller than adult females, which are uniformly drab olive (Appendix Figure 1). Males possess individual unique body ornamentation patterns, comprised of patches of orange/yellow, black (melanin) and iridescent colours (Houde 1997; Appendix Figure 2). All fish used for this thesis originated from the Upper

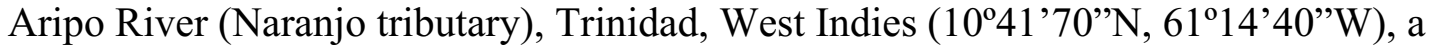
low-predation population (Magurran 2005; Appendix Figure 3).

The guppy is an ideal species to study the role of social information in mate choice, social partner choice and sexual selection for the following reasons. Guppies exhibit a non-resource based, promiscuous mating system wherein both males and females exert mate choice. Male guppies (Dosen and Montgomerie 2004a; Herdman et al. 2004a,b), like males in many species (Andersson 1994; Bonduriansky 2001; CluttonBrock 2007; Edward and Chapman 2011), tend to prefer larger, more fecund females as mates. Preferentially mating with large and fecund females can potentially confer greater reproductive success to males that may offset the costs of mate choice (Parker 1983; Servedio and Lande 2006; Edward and Chapman 2012), subject to the constraints of sperm competition and cryptic female choice (Wedell et al. 2002). Male guppies also prefer to mate with sexually receptive females (Guevara-Fiore et al. 2009). 
In natural guppy populations, males and females co-occur in mixed-sex shoals (Houde 1997) wherein there are opportunities for both males and females to use social information extracted from the mating behaviour of other nearby individuals. Males devote most of their time sexually pursuing females and females spend the majority of their time foraging (Houde 1997). Non-pregnant, sexually-receptive females are generally rare (Houde 1997) and the operational sex ratio is thus commonly male biased (Houde 1997; Pettersson et al. 2004). In addition, most adult females are multiply mated in natural populations (Kelly et al. 1999; Neff et al. 2008) and are able to store sperm for up to six months after insemination (Houde 1997). This, coupled with its promiscuous mating system and vigorous sexual behaviour, results in a high level of sexual and sperm competition in this species (Matthews et al. 1997; Pilastro and Bisazza 1999; Matthews and Magurran 2000; Neff et al. 2008). Consequently, the costs to males of locating receptive and(or) high-quality mates are likely high and social information obtained from sexual interactions between conspecifics could potentially be used to reduce such costs. Male guppies are sensitive to perceived ambient changes in their risk of sperm competition (Dosen and Montgomerie 2004b; Jeswiet et al. 2011b, 2012), and alter their mating behaviour in the presence of one potential sexual competitor (Makowicz et al. 2010b; Jeswiet et al. 2011b, 2012; Bierbach et al. 2013; Auld et al. 2015; Chapter 6). Adult male guppies are plastic in their mating behaviour and are individually capable of exhibiting two alternative mating tactics (Houde 1997; Chapter 8), which are the principle components of their mating effort (Godin and Auld 2013; Chapter 4). They can achieve copulations and potential fertilizations either by soliciting a female using courtship displays or circumventing pre-copulatory female choice through sneak 
(coerced) copulations (Houde 1997). When courting a female, a male performs sigmoid courtship displays by bending his body into an ' $\mathrm{S}$ ' shape and quivering in front of the female; courtship can lead to consensual mating (Houde 1997). A sneak copulation (or gonopodial thrusting) attempt involves a male approaching a female from behind or laterally, rapidly darting towards her with his gonopodium extended anteriorly, and attempting to insert the tip of the gonopodium into the female's gonopore to transfer sperm (Houde 1997). Females typically avoid sneak-copulating males (Houde 1997) and are able to exert some control over fertilization post-copulation via cryptic female choice (Pilastro et al. 2004). On average, courtship displays in male guppies are significantly longer in duration, lead to more sperm being inseminated and achieve greater insemination success than sneak mating attempts (Pilastro and Bisazza 1999). Previous studies have demonstrated plasticity in the use of alternative mating tactics by male Trinidadian guppies in different contexts, such as predation (Godin 1995), varying ambient light levels (Chapman et al. 2009), varying food availability and parasite infection (Kolluru and Grether 2005; Kolluru et al. 2009).

\subsubsection{Wild-caught fish}

I used free-ranging adult guppies, collected haphazardly by hand seining from the Upper Aripo River (Naranjo tributary), for the experiments described in Chapters $4-8$. The fish were transported in containers of aerated river water to a laboratory at the University of the West Indies, St. Augustine ( $1 \mathrm{~h}$ transit time), where they were held in mixed-sex aquaria filled with filtered aged tap water $\left(24-26^{\circ} \mathrm{C}\right)$ and illuminated overhead with fluorescent lighting and diffused natural lighting. I fed them ad libitum 2-3 times daily 
with commercial flake food (Nutrafin ${ }^{\mathrm{TM}}$ ) and live brine shrimp nauplii (Artemia franciscana). Whilst in holding aquaria, the fish were treated with antifungal (Fungus Guard $^{\mathrm{TM}}$, Pimafix, Melafix) and antiparasitic (Life Bearer ${ }^{\mathrm{TM}}$ ) medicine, and were thus free of any fungus or ectoparasites prior to experimentation.

For the experiments described in Chapters 4 - 8, I followed Dosen and Montgomerie (2004) and used gravid (pregnant) females, who are generally unresponsive to male courtship and copulation attempts (Houde 1997), as stimulus fish to ensure that male mate choice would not be confounded by female sexual responses to male sexual activity and to minimize variation in male behaviour caused by any differences in female reproductive state. Even when females are not sexually receptive, Trinidadian male guppies sexually pursue, court and attempt to mate with gravid females in both the wild and the laboratory (Houde 1997; Guevara-Fiore et al. 2009; Jeswiet and Godin 2011; Godin and Auld 2013) and can successfully inseminate unreceptive gravid females through forced sneak copulations (Pilastro and Bisazza 1999; Evans et al. 2003a) and sire offspring (Kelly et al. 1999; Herdman et al. 2004; Neff at al. 2008).

The day before mate choice trials, males were isolated from females to allow them sufficient time to replenish their sperm reserves and to ensure that all test males were similarly sexually motivated at the onset of the behavioural trials.

\subsubsection{Laboratory-reared fish}

Because female guppies are most strongly receptive to male courtship when they are virgins or for a brief period after parturition (Houde 1997) and prior mating experience can affect mate choice (Pitcher et al. 2003), I appropriately used laboratory-born and - 
reared virgin females for the experiments described in Chapters 3 and 9. Study subjects were second- to fourth-generation descendants of adult guppies collected haphazardly by hand seining from the Upper Aripo River (Naranjo tributary) and held in mixed-sex breeding aquaria in a laboratory at Carleton University. To ensure that test females were virgin, and thus sexually receptive, and to prevent prior mating, newborn fish were removed from mixed-sex breeding aquaria and placed in juvenile rearing aquaria and monitored daily for signs of sexual differentiation, as noted by the ontogenetic appearance of the gonopodium (the sperm intromittant organ) and(or) body ornamentation in males (at approximately 4 weeks of age). Upon such identification, sexually mature males were removed from their juvenile rearing aquarium and placed in separate male-only glass home aquaria. The positions of male-only and female-only home aquaria on staging shelves were alternated to ensure that both males and females could view each other and thus acquire prior visual contact with the opposite sex. All aquaria were filled with filtered aged tap water $\left(24-26^{\circ} \mathrm{C}\right)$ and illuminated overhead with fluorescent lighting on a $13 \mathrm{~h} \mathrm{~L}: 11 \mathrm{~h}$ D cycle. Fish were fed ad libitum 2-3 times daily with commercial flake food (Nutrafin ${ }^{\mathrm{TM}}$ ) and live brine shrimp nauplii (Artemia franciscana).

\subsection{General experimental protocol}

Because guppies can become familiar with each other after 12 days of association (Griffiths and Magurran 1997) and social familiarity can potentially affect mate choice in both the absence (Hughes et al. 1999; Kelley et al. 1999) and presence of an audience (Bierbach et al. 2011a), for all experiments described herein I selected males and females 
in any given mate-choice trial from different holding aquaria as they were presumably unfamiliar with each other prior to testing. Paired stimulus females used in experiments described in Chapters 4 - 7 were gravid and matched as closely as possible for their degree of abdominal distension (assessed by eye), an indicator of stage of pregnancy (Houde 1997).

For experiments in Chapters 3, 5, 6, 7 and 9, I used dichotomous choice tests to test for a focal fish's preference to associate with either of two stimulus fish presented simultaneously (see individual chapters for specific details). I recorded the time that the focal fish spent in the preference zone near (within $10 \mathrm{~cm}$ ) each stimulus fish, and facing $\left(0 \pm 90^{\circ}\right)$ the stimulus fish, as a proxy measure of his mating or social partner preference, depending on the experiment. Such association time in dichotomous-choice test is a known reliable predictor of mating preference in the guppy (Dugatkin and Godin 1992; Jeswiet and Godin 2011) and other species (Witte 1996b, Lehtonen and Lindström 1998; Walling et al. 2010), as well as social partner choice in the guppy (Lachlan et al. 2008). To control for the possibility that the focal fish might prefer a certain side of the test aquarium rather than a particular stimulus fish (i.e. a side bias), I switched the positions of the two end compartments that held the stimulus fish at the end of the first half of a 20min choice test (i.e. Preference test 1), following which the second half of the test (i.e. Preference test 2) was carried out; see individual chapters for specific details. To calculate an overall mating or social preference score for each test fish (depending on the experiment), I summed the amount of time that the fish spent associating with each stimulus fish in both preference tests. I also summed the total time that each focal fish 
spent on each side of the test aquarium over both preference tests, irrespective of the identity of the stimulus fish.

A side bias was defined as a focal fish spending more than $80 \%$ of his or her total association time in the same preference zone (i.e. same side of aquarium), even after the positions of the end compartments containing the stimulus fish had been switched (cf. Schlupp and Ryan 1997; Dosen and Montgomerie 2004a). A trial was discarded from further analysis if the focal fish exhibited a side bias.

\subsection{General experimental apparatus}

In all experiments, test aquaria contained a layer of natural river gravel on the bottom, and were filled with aerated aged tap water $\left(15 \mathrm{~cm}\right.$ depth, $\left.24-26^{\circ} \mathrm{C}\right)$ and illuminated by overhead fluorescent lights and diffused natural light (except for the experiments described in Chapters 3 and 9, where only fluorescent lights illuminated the aquaria). For experiments involving a dichotomous choice test (Chapters 3, 5, 6, 7, 9), vertical lines drawn on the front and back walls of central test aquaria demarcated a $10-\mathrm{cm}$ wide mating or social preference zone near each of the end compartments. The back wall of the test aquaria and three sides of the stimulus compartments were covered externally with tan paper to reduce external disturbances and to provide a uniform background. In Chapters 4 and 8, there were no end compartments present and so all three sides of the test aquaria were covered with tan paper. In all experiments, the behaviour of focal fish was observed from behind a blind. 


\subsection{Measurement of fish body length and ornamentation}

At the end of each behavioural trial for all experiments, all fish were lightly anaesthetized with MS-222 and their left side photographed next to a ruler using a digital camera. From the photographs, I measured the standard body length and area of the left side of the body (excluding the fins) for all fish, and the body ornamentation pattern of all males using Image $J$ software (http://rsbweb.nih.gov/ij/). I quantified the areas of black and orange pigmentation, which are two sexually-selected male traits in the guppy (reviewed in Houde 1997; see also Chapter 3), on the left side of each male's body, excluding the fins. A male's body colour score was expressed as the relative area of total body colour ([black area + orange area]/total body area) to control for variation in male body size. 


\section{Chapter 3:}

\section{Independent Mating Preferences for Male Body Size and Ornamentation in Female Trinidadian Guppies}

This chapter is published as: Auld, H.L., Pusiak, R.J.P. and Godin, J.-G.J. 2016.

Independent mating preferences for male body size and coloration in female Trinidadian guppies. Ethology, 122: 597-608. It is reproduced here with permission from WileyBlackwell Publishing ${ }^{\circ}$. 


\subsection{Abstract}

Although females in numerous species generally prefer males with larger, brighter and more elaborate sexual traits, there is nonetheless considerable intra- and inter-population variation in mating preferences amongst females that requires explanation. Such variation exists in the Trinidadian guppy, Poecilia reticulata, an important model organism for the study of sexual selection and mate choice. While female guppies tend to prefer more ornamented males as mates, particularly those with greater amounts of orange coloration, there remains variation both in male traits and female mating preferences within and between populations. Male body size is another trait that is sexually selected through female mate choice in some species, but has not been examined extensively in the guppy despite known intra-and inter-population variation in this trait among adult males and its importance for survivorship in this species. In the current study, I used a dichotomouschoice test to quantify the mating preferences of female guppies, originating from a lowpredation population in Trinidad, for two male traits, body length and area of the body covered with orange and black pigmentation, independently of each other. I expected strong female mating preferences for both male body length and ornamentation in this population, given relaxation from predation and presumably relatively low cost of choice. Females indeed exhibited a strong preference for larger males as expected, but surprisingly a weaker (but nonetheless significant) preference for orange and black ornamentation. Interestingly, larger females demonstrated stronger preferences for larger males than did smaller females, which could potentially lead to size-assortative mating in nature. My results thus contribute further to the understanding of both intra- and interpopulation variation in mating preferences in the Trinidadian guppy. 


\subsection{Introduction}

When choosing amongst potential mates, females often use multiple physical and (or) behavioural traits in males, which may be honest indicators of overall male quality (Jennions and Petrie 1997; Candolin 2003). In populations wherein males experience high levels of competition for mating opportunities, such as those with male-biased operational sex ratios or promiscuous mating systems, males tend to exhibit elaborate or exaggerated secondary sexual traits despite their associated costs (e.g. increased energetic expenditure, predation risk) (Andersson 1994; Andersson and Simmons 2006). Although females across a wide range of taxa exhibit an overall general preference for males who possess highly elaborate physical and (or) behavioural traits, such as bright colours (e.g. Kemp 2007; Hill 2006), large body size (e.g. Partridge et al. 1987; Brown 1990; Gilburn et al. 1992; Pyron 2003) and courtship displays (e.g. Wagner et al. 2000), there is nonetheless considerable variation in the strength and direction of female mating preferences both within and between populations that require explanation (Jennions and Petrie 1997). Understanding how variation in fitness-related traits, including behaviour, that are under selection is maintained in the wild has been an important and enduring challenge (Fisher 1930).

Observed variation in female mating preferences can be the result of differences between individuals in genotype and gene expression (Brooks and Endler 2001; Pitcher et al. 2008; Cummings 2015), body condition (Syriatowicz and Brooks 2004; Hunt et al. 2005), age (Kodric-Brown and Nicoletto 2001a), reproductive state (Lynch et al. 2005) and (or) behavioural plasticity that occur either across the life span of a female or as the result of females optimizing mate choice in dynamic environments (Vakirtzis 2011). 
Although genetic variation in female mate preferences between populations has been linked to variation in ecological and environmental factors, including predation (Breden and Stoner 1987; Godin and Briggs 1996), food availability (Grether 2000) and water turbidity (Heuschele et al. 2009), there remains unexplained variation between populations that is not accounted for by any of these factors individually. Additionally, in species with mutual mate choice, mating preferences can be constrained by the mating preferences of the opposite sex, which can lead to positive size-assortative mating for example (e.g. Olsson 1993; Baldauf et al. 2009; Holveck and Riebel 2010; Jiang et al. 2013; Ingram et al. 2015). Because the ecological selective factors that are known to shape mating preferences can vary geographically (Endler 1995), population-specific mating preferences can evolve over time (Houde and Endler 1990; Endler and Houde 1995) and potentially result in speciation (Rundle and Boughman 2010).

Previous studies on the guppy have shown female preference for multiple secondary sexual traits in males, including tail size (e.g. Bischoff et al. 1985), colour spots, particularly orange and black pigmentation (reviewed in Houde 1997; Magurran 2005), body size (e.g. Reynolds and Gross 1992; Brooks and Endler 2001; Watt et al. 2001), male courtship display rate (e.g. Kodric-Brown 1993; Kodric-Brown and Nicoletto 2001b) and personality (Godin and Dugatkin 1996), depending on the population. Because natural populations of guppies in Trinidad are subject to different ecological factors, such as predation (Endler 1995; Magurran 2005), and environmental factors, such as water turbidity, canopy cover and available nutrients (Endler 1995; Grether et al. 2005), which can affect male sexual traits and female mating preferences for those traits, population differences in female mating preferences are to be expected. 
Indeed, while female guppies tend to prefer males with greater ornamentation in most populations studied to date (reviewed in Houde 1997; Magurran 2005), individuals are not unanimous in their mate choice (Endler and Houde 1995; Houde and Hankes 1997; Brooks and Endler 2001), which vary both between (e.g. Houde 1988a,b; Endler and Houde 1995; Houde and Endler 1990; Brooks 2002) and within populations (e.g. Godin and Dugatkin 1995).

Compared with male body ornamentation as a sexually-selected trait, female mate choice based on male body size has not been examined extensively in the Trinidadian guppy and results from the few existing studies vary. More specifically, although female mate preference for larger males over smaller ones has been demonstrated in guppies from different populations in Trinidad (Reynolds and Gross 1992; Magellan et al. 2005) or from unknown source populations (Watt et al. 2001; Zajitschek et al. 2006), Schwartz and Hendry (2007) did not detect female preferences for either male body size or ornamentation in any of the low-predation Trinidadian populations they studied. Moreover, potentially important interactions between male body ornamentation and body size in shaping female mating preferences within populations remain largely unexplored. Although multiple female preferences for male traits can evolve independently from each other in the guppy (Brooks 2002), little work has been done to compare the relative strength of female preferences for independent male traits. Female mating preferences (Houde 1988a,b; Brooks and Endler 2001), male colour pattern (Houde 1992) and male body size (Karino and Haijima 2001) are all variable and heritable in the guppy and thus responsive to selection (Endler 1980; Houde and Endler 1990; Houde 1994; Reznick et al. 1997). 
Therefore, in the current study, I tested virgin female guppies descended from adults collected in a low-predation population in Trinidad for their mating preferences based on male body length and colour ornamentation, independently of each other. Female guppies that have evolved under weak predation pressure should express relatively strong mating preferences (Endler and Houde 1995), including preferences for larger and more ornamented males (Endler and Houde 1995; Magellan et al. 2005), as the costs associated with active mate choice under relaxed predation should be less than in high-predation environments (Godin and Briggs 1996). I thus predicted that females originating from this low-predation population would exhibit, on average, mating preferences for larger males over smaller ones and for more ornamented males over less ornamented ones when given a choice. I quantitatively assessed and compared the relative strength of female preferences for male body length and colour ornamentation. Because female mating preference is likely to vary with female quality (Cotton et al. 2006), and female fecundity (i.e. quality) is positively correlated with body length in adult female guppies (Herdman et al. 2004), I also characterized the relationships between the body length of individual females and the strength of their mating preferences based on male body length and ornamentation separately, which are presently unknown.

\subsection{Methods}

\subsubsection{Study animals}

I used laboratory-reared Trinidadian guppies as a study species. See Chapter 2 for a rationale. 


\subsubsection{General protocol}

See Chapter 2 for a description of the common general experimental protocol.

\subsubsection{Experimental apparatus}

The experimental apparatus (Figure 3-1) consisted of a clear Plexiglas test aquarium (40 x 20 x $25 \mathrm{~cm}$; L x W x H) flanked by a clear Plexiglas container $(15 \times 20$ x $25 \mathrm{~cm}$; hereafter termed compartment) at either end. The central aquarium served as the matechoice arena and held the focal female, and the end compartments each contained a stimulus male. Since chemical cues can also influence mate choice in animals (Candolin 2003), placing the stimulus males in the end compartments outside the central test aquarium and separated from the test female prevented any exchange of chemical cues between them and ensured that females could only use visual cues when assessing the stimulus males as potential mates. The experimental apparatus was illuminated overhead with a single Sun-Glo ${ }^{\mathrm{TM}}$ broad-spectrum fluorescent tube $\left(\mathrm{T} 8,4200^{\circ} \mathrm{K}\right.$, R.C. Hagen Inc., Montréal) that generated a light irradiance of $8 \mu \mathrm{M} / \mathrm{m}^{2} / \mathrm{s}(400-700 \mathrm{~nm}$, measured with a Apoge MQ-200 quantum radiometer) at the water surface. This irradiance level falls within the range of ambient light irradiances observed in early-mid morning and mid-late afternoon on upland forest streams in Trinidad (Endler 1991; Grether et al. 2001), times of day when guppy courtship activity is maximal (Endler 1987). The maximum spectral sensitivity of the guppy retina ranges between $400-600 \mathrm{~nm}$ (Endler 1991), which overlaps with most of the irradiance spectrum of the fluorescent tube illuminating the test aquarium. See Chapter 2 for more information on general test aquaria conditions. 


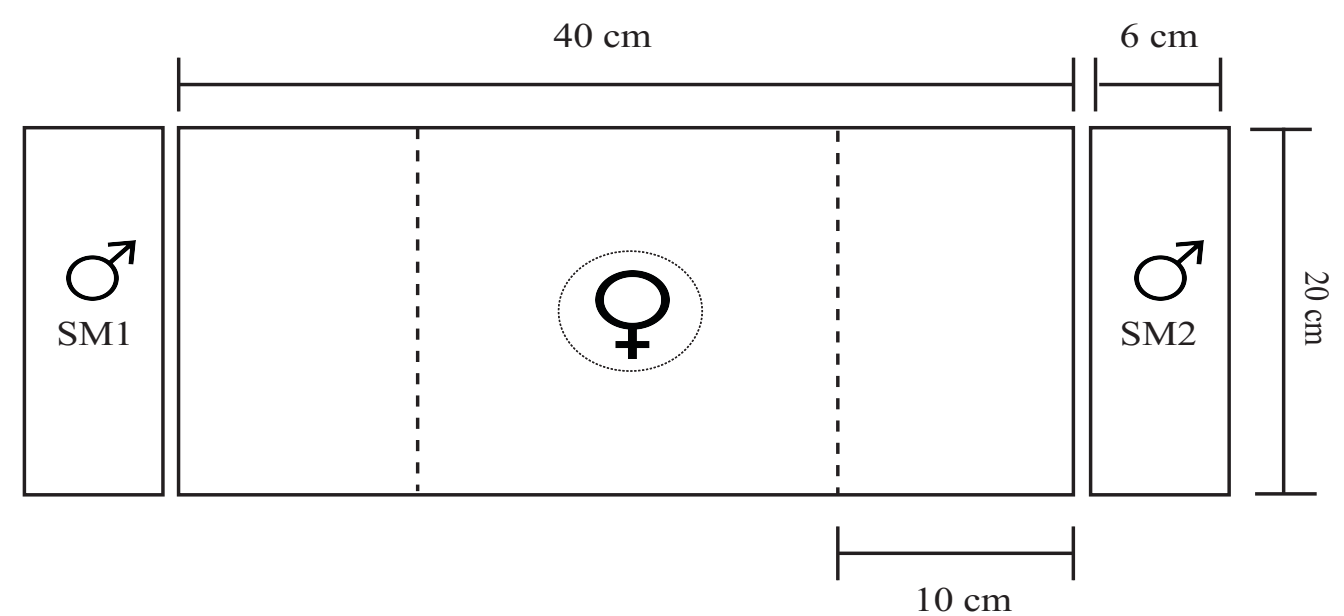

Figure 3-1 Schematic top view of the experimental apparatus (not to scale). The test aquarium ( $40 \times 20 \times 25 \mathrm{~cm}$; $\mathrm{L} \mathrm{x} \mathrm{W} \mathrm{x} \mathrm{H)} \mathrm{held} \mathrm{the} \mathrm{focal} \mathrm{female} \mathrm{and} \mathrm{the} \mathrm{end} \mathrm{compartments}$ (6 $\times 20 \times 22 \mathrm{~cm}$ held the stimulus males (SM1 and SM2)). The end compartments held one stimulus male each. One stimulus male was either larger or more colour ornamented than the other stimulus male. The stippled circle represents a removable clear Plexiglas cylinder ( $7 \mathrm{~cm}$ diam.), in which the focal female could temporarily be placed. The dashed lines demarcate $10-\mathrm{cm}$ mating preference zones. 


\subsubsection{Experimental protocol}

I quantified the mating preference of individual focal females for either of two stimulus males using a dichotomous-choice paradigm, commonly used for studying mate choice (cf. Dugatkin and Godin 1992; Kodric-Brown and Nicoletto 2001b; Jeswiet and Godin 2011). For any given mate-choice trial, a focal female was given a choice between two stimulus males that differed in their predicted sexual attractiveness to varying degree (see below for methodology). I used the time that a focal female spent associating with either stimulus male as a proxy of her mating preference. Such association time is a known reliable predictor of mating preference in both female (Dugatkin and Godin 1992; Kodric-Brown 1993) and male (Jeswiet and Godin 2011) guppies, as well as in other species (e.g. Witte 2006b; Lehtonen and Lindström 2008; Walling et al. 2010). The experiment comprised two treatments, which decoupled female mating preferences for male body length and male body ornamentation. In Treatment 1 , the mating preference of focal females $(N=32)$ for males based on body length was tested by presenting individual females with two stimulus males that were matched for body ornamentation (orange and black pigmentation), but differed significantly in body length (Table 3-1). In Treatment 2 , focal females $(N=29)$ were presented with two stimulus males that were matched for body length, but differed significantly in body ornamentation (Table 3-2). When selecting paired stimulus males for each trial, male body ornamentation (proportion of their body covered with orange and black pigmentation) and body length were initially determined as closely as possible by eye and with a metric ruler, respectively. Both the body length and ornamentation of individual males were later quantified more accurately from their digital photographs (see Chapter 2 for more details 
of the method used; Tables 3-1 and 3-2). In the Upper Aripo River (Naranjo tributary) population, male body length and colour score are only weakly correlated (Pearson's $r=$ $0.152, N=672)$, as are body areas covered by orange and black pigmentation $(r=0.126$, $N=575$; Auge et al. 2016). 
Table 3-1 Mean \pm SE total body length $(\mathrm{mm})$ of focal test females and total body length and body colour score (proportion of left side of body covered with black and orange colours) of the paired stimulus males categorized as relatively more attractive [(MA); either larger or more colour ornamented] and less attractive [(LA); either smaller or less colour ornamented] used in each experimental treatment. The body length and colour scores of paired stimulus males in each treatment were separately compared using the paired $t$ test. $N$ denotes the number of independent replicated trials in each treatment, and the numbers in brackets are the range. Colour scores were highly repeatable (Pearson's $r=0.94, P<0.001, d f=148)$.

\begin{tabular}{|c|c|c|c|c|c|c|}
\hline & Trait & Focal female & MA male & LA male & $\begin{array}{l}\text { Difference } \\
|\mathrm{MA}-\mathrm{LA}|\end{array}$ & Paired $t$-test \\
\hline \multirow[t]{3}{*}{$\begin{array}{l}\text { Treatment } 1 \\
\quad(N=32)\end{array}$} & Body length & $\begin{array}{c}23.4 \pm 0.5 \\
(18.1-30.2)\end{array}$ & $\begin{array}{c}18.7 \pm 0.2 \\
(16.3-20.2)\end{array}$ & $\begin{array}{c}15.6 \pm 0.1 \\
(14.2-17.3)\end{array}$ & $3.12 \pm 0.19$ & $t_{31}=16.58, P<0.001$ \\
\hline & Orange colour & - & $\begin{array}{c}1.77 \pm 0.18 \\
(0.25-3.48)\end{array}$ & $\begin{array}{c}1.84 \pm 0.21 \\
(0.27-5.35)\end{array}$ & $0.07 \pm 0.25$ & $t_{31}=0.29, P=0.77$ \\
\hline & Black colour & - & $\begin{array}{l}1.99 \pm 0.22 \\
(0.24-6.0)\end{array}$ & $\begin{array}{c}2.14 \pm 0.23 \\
(0-7.04)\end{array}$ & $0.15 \pm 0.32$ & $t_{31}=0.45, P=0.65$ \\
\hline \multirow[t]{3}{*}{$\begin{array}{l}\text { Treatment } 2 \\
(N=29)\end{array}$} & Body length & $\begin{array}{c}21.7 \pm 0.4 \\
(18.5-25.4)\end{array}$ & $\begin{array}{c}16.2 \pm 0.2 \\
(15.0-18.9)\end{array}$ & $\begin{array}{c}16.2 \pm 0.2 \\
(14.5-18.7)\end{array}$ & $0.006 \pm 0.18$ & $t_{28}=0.29, P=0.98$ \\
\hline & Orange colour & - & $\begin{array}{c}3.29 \pm 0.28 \\
(1.46-9.30)\end{array}$ & $\begin{array}{c}1.44 \pm 0.21 \\
(0-3.50)\end{array}$ & $1.86 \pm 0.38$ & $t_{28}=4.83, P<0.001$ \\
\hline & Black colour & - & $\begin{array}{c}2.62 \pm 0.23 \\
(0.26-6.39)\end{array}$ & $\begin{array}{c}1.09 \pm 0.17 \\
(0-3.37)\end{array}$ & $1.54 \pm 0.22$ & $t_{28}=6.92, P<0.001$ \\
\hline
\end{tabular}


For each mate-choice trial, a female (mean \pm SE standard length $(\mathrm{SL})=23.4 \pm 0.5$ $\mathrm{mm}$, range $=18.1-30.2 \mathrm{~mm}, N=86)$ was dip-netted haphazardly from an all-female holding aquarium, measured for standard length (from the tip of the lower lip to the end of the caudal peduncle) using a metric ruler, and then gently placed into the central test aquarium. Two stimulus males were then selected from an all-male holding aquarium and each one placed in one or the other of the end compartments, determined at random.

Before testing, all fish were given a 30-min acclimatization period during which the focal female was able to swim freely about the central test aquarium. Visual contact between the stimulus males and the test female during this period was prevented by opaque Plexiglas screens placed between the end compartments and the central test aquarium (Figure 3-1). At the end of the acclimatization period, the female was gently dip-netted and placed into a clear Plexiglas cylinder $(7 \mathrm{~cm}$ diam.) located in the centre of the test aquarium and the opaque screens were slowly removed, allowing the focal female to view both stimulus males for $10 \mathrm{~min}$. After this viewing period, the central cylinder was slowly raised, allowing the female to swim freely about the test aquarium and to choose between the two stimulus males. Once the female resumed swimming normally, I carried out an initial 10-min preference test (Preference test 1), during which the amount time that the focal female spent in the associated $10-\mathrm{cm}$ preference zone and facing $( \pm$ $90^{\circ}$ ) a particular stimulus male (i.e. association time) was recorded using stopwatches as a proxy of her preference for that male. At the end of Preference test 1, the opaque screens were gently replaced, again preventing visual contact between the test female and the stimulus males, and the test female temporarily returned to the clear Plexiglas cylinder in the centre of the test aquarium. 
To control for the possibility that the test female might prefer a certain side of the test aquarium rather than a particular male (i.e. a side bias), I switched the positions of the two end compartments that held the stimulus males at the end of Preference test 1. The fish were then given an additional 2-min re-acclimation period, following which the opaque screens were slowly removed and the female released from the central cylinder. Once the female resumed swimming, a second 10-min preference test (Preference test 2) was carried out, as described above for Preference test 1 . To calculate an overall mating preference score for each test female, I summed the amount of time that the female spent associating with each male in both preference tests. I also summed the total time that each female spent on each side of the test aquarium over both preference tests, irrespective of the identity of the stimulus male. In twenty-four such trials (out of an initial total of 86 trials), focal females demonstrated a side bias and were discarded. An additional trial was discarded when, upon more accurate measurement, I discovered that one of the stimulus males was less than $14 \mathrm{~mm} \mathrm{SL}$ and not likely sexually mature. The 61 remaining trials ( $N$ $=61$ focal females) were used for the analysis. I used different focal females and stimulus males for each trial.

\subsubsection{Statistical analysis}

All statistical analyses were carried out using R statistical software (R Development Core Team 2012).

I first ascertained whether focal females expressed non-random mating preferences. I specifically tested the a priori prediction that females would spend more time associating with the male categorized as the larger or more colour ornamented of the two stimulus males than expected by chance using a two-tailed, one-sample $t$-test for both 
Treatments 1 and 2 separately. To estimate effect sizes, I calculated Cohen's $d$ and associated $95 \%$ confidence intervals from the $t$-values using the R package 'MBESS' (Kelley 2007a,b; Kelley and Lai 2012). Second, for each treatment separately, I used linear regressions to ascertain whether $(i)$ the strength of the mating preference of focal females for the larger or more ornamented male and (ii) their sexual motivation (as as measured by total time spent in the preference zone of either stimulus male) in each trial covaried with their individual body length. To estimate effect sizes, I calculated Cohen's $d$ and associated confidence intervals from the regression coefficients (Pearson's $r$ ) obtained using the R package ‘compute.es' (Del Re 2013). Residuals from the models were examined for normality using Q-Q plots. If they were not normally distributed, then the proportional data were arcsine transformed and(or) the continuous variables were log transformed.

\subsubsection{Ethical standards}

This study was approved by the institutional Animal Care Committee at Carleton University (protocol 13281), and thus conforms to the requirements of the Canadian Council on Animal Care and the laws of Canada regarding animal use and care and adheres to the guidelines for the use of animals in research of the Animal Behavior Society. 


\subsection{Results}

As predicted, focal females exhibited on average significant mating preferences for the larger of the two stimulus males (who were matched for colour score) in Treatment 1 (one sample $t$-test, $t_{31}=8.25, P<0.001, d=1.46$ [CI: 0.95, 1.95]; Figure 3-2a) and for the more ornamented of the two stimulus males (who were matched for body length) in Treatment $2\left(t_{28}=2.39, P=0.02, d=0.44\right.$ [CI: 0.06, 0.82]; Figure 3-2a), when compared to that expected by chance. Comparing these non-overlapping effect sizes, the strength of female mating preferences based on male body length is significantly greater ( $>3$ fold) than that for male colour ornamentation. In Treatment 2 , there was no difference in the observed significant preference of females for the more ornamented of the paired stimulus males (who were matched for body length) based on their orange coloration (one-sample $t$-test, $t_{28}=3.43, P=0.002, d=0.64$ [CI: $\left.0.23,1.03\right]$; Figure $3-2 b$ ) of the males compared to their black coloration $\left(t_{28}=2.09, P=0.05, d=0.38\right.$ [CI: 0.007, 0.76]; Figure 3-2b), as revealed by the overlapping confidence intervals of their respective effect size.

Interestingly, the strength of female preference for the larger of the two stimulus males tended to increase, although marginally not significantly so, with the body length of individual focal females in Treatment 1 (linear regression, $t_{30}=1.88, P=0.07, d=$ 0.69 [CI: $-0.09,1.46]$; Figure 3-3a). In contrast, preference for the more ornamented of the paired stimulus males did not vary with focal female body length in Treatment 2 (linear regression, $t_{27}=-1.22, P=0.24, d=-0.47$ [CI: $-1.27,0.32$ ]; Figure $3-3 b$ ). Overall sexual motivation of focal females, as measured by total time spent in the preference zone of either stimulus male, either did not vary significantly (linear regression, 
Treatment $2, t_{27}=-0.84, P=0.41, d=-0.32$ [CI: $\left.-1.11,0.46\right]$ ) or marginally decreased (Treatment $1, t_{30}=-2.08, P=0.05, d=-0.76$ [CI: $\left.-1.54,0.03\right]$ ) with female body length. 


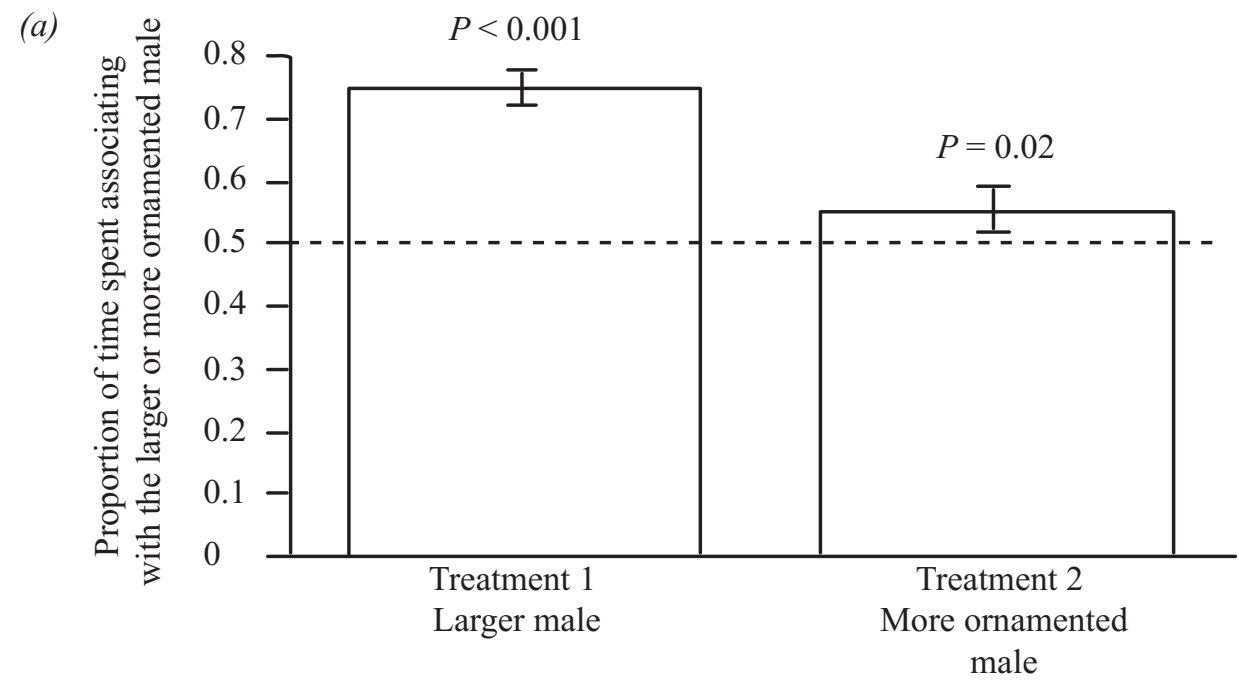

(b)

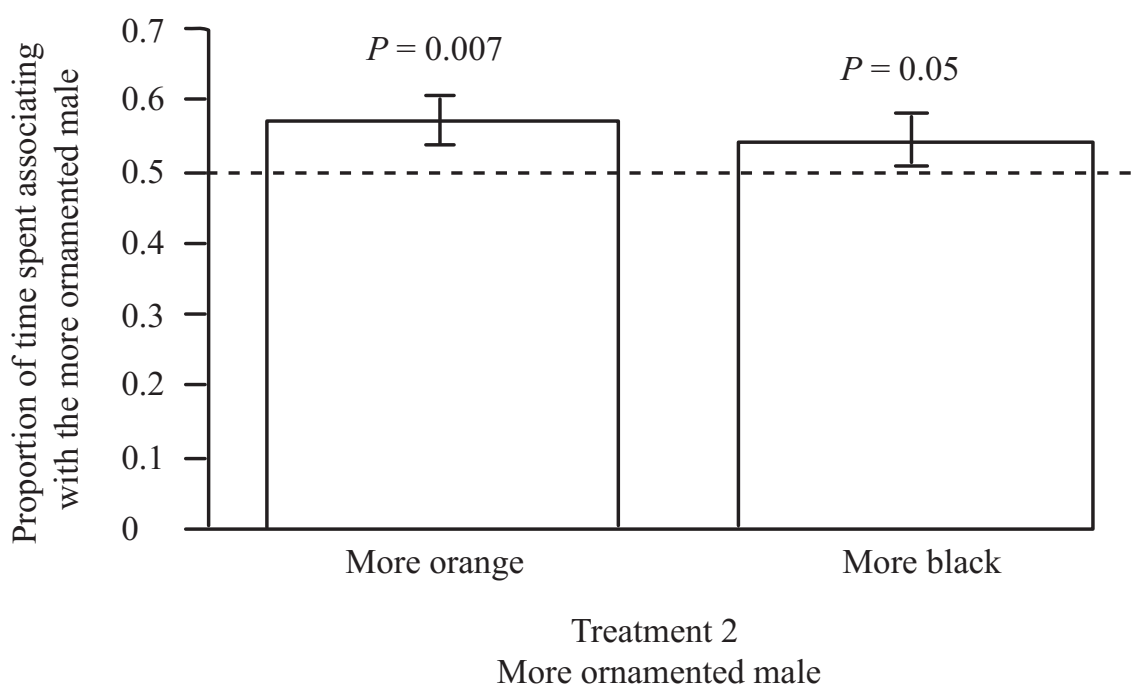

Figure 3-2 Mean \pm SE female preference (= proportion of total association time spent near) for (a) the larger or more colour ornamented male in Treatments 1 and 2, respectively, and (b) the more colour ornamented male in Treatment 2 with a greater proportion of his body covered with orange and black pigmentation, separately. The horizontal stippled lines at proportion 0.5 denote no choice (i.e. chance level). 


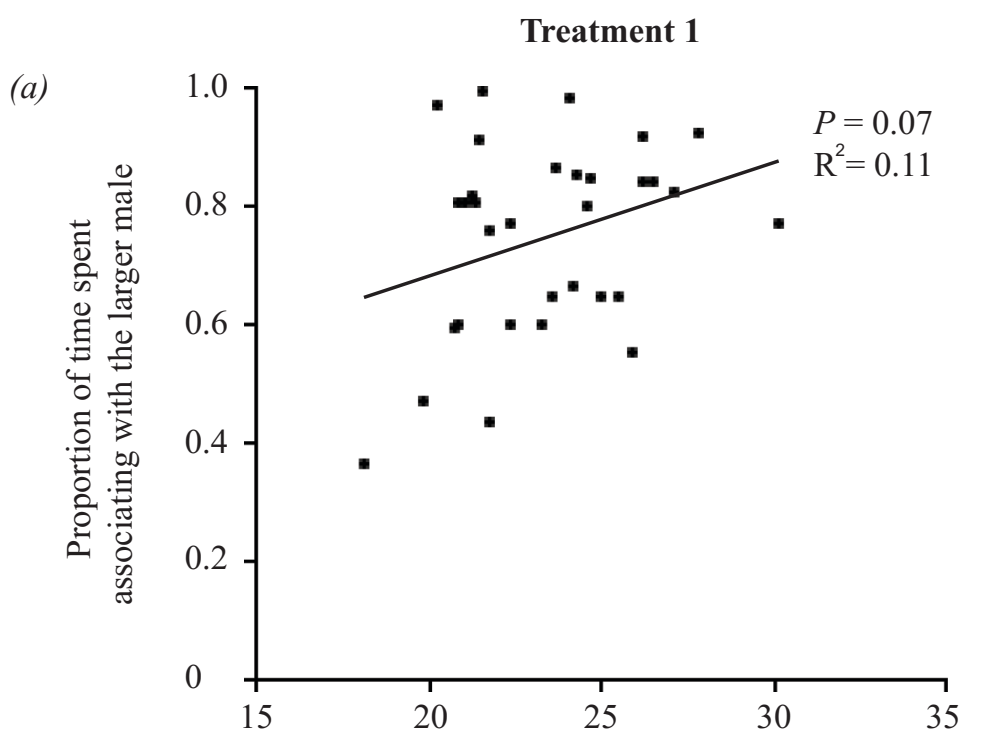

(b)

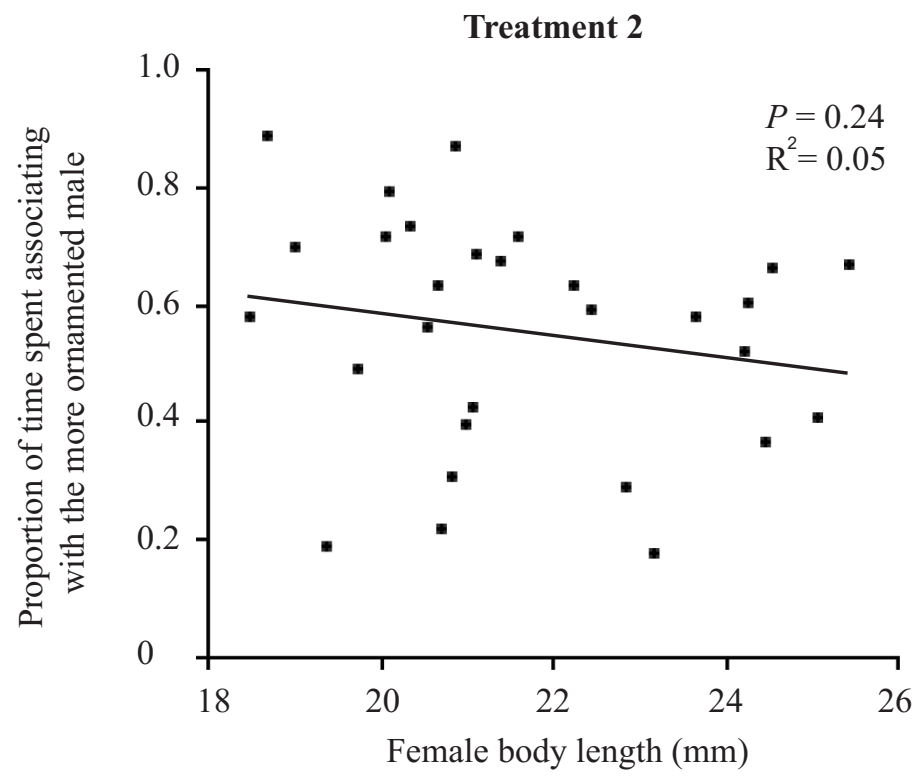

Figure 3-3 The proportion of time focal females spent in the preference zone of the (a) larger or (b) more colour ornamented of the paired stimulus males as a function of female body length. In Treatment 2 (panel $b$ ), male ornamentation is the proportion of their body covered in orange and black pigmentation. Non-transformed data and linear regression lines are shown. 


\subsection{Discussion}

As predicted, when provided with the opportunity to choose between two stimulus males that differed in either body length or ornamentation, virgin female guppies descended from a low-predation population in Trinidad preferred on average larger over smaller males when the males were matched for body ornamentation, and also exhibited a significant, but weaker, preference for more ornamented males (based on orange and black colour) when paired stimulus males were size matched.

Female preference for more ornamented males has previously been observed for several guppy populations in Trinidad (reviewed in Houde and Endler 1990; Endler and Houde 1995; Houde 1997); my results corroborate this general preference in the Upper Aripo (Naranjo tributary) guppy population. In comparison, there are relatively few experimental studies of female mating preferences based on male body size in the guppy, with the majority of them revealing female preferences for larger males. More specifically, female guppies descended from a high-predation population in Trinidad (Reynolds and Gross 1992) and feral female guppies descendant from unknown Trinidadian source populations (Watt et al. 2001; Zajitschek et al. 2006) have been shown to prefer larger over smaller males as mates. The latter findings are corroborated by my current results and those of Magellan et al. (2005), based on guppies originating from the low-predation Upper Aripo population by comparison. It is unknown whether the guppies used by Magellan et al. (2005) originated from the same tributary (Naranjo tributary) of the Upper Aripo River as my fish. Mating with larger males can provide indirect fitness benefits to females via more successful offspring (Reynolds and Gross 1992; Watt et al. 2001). Larger males produce larger offspring, given that body size is 
heritable in the guppy (Karino and Haijima 2001). Large body size in offspring confers greater survivorship (Reynolds and Gross 1992; Watt et al. 2001) and potentially a competitive mating advantage to males, as larger male guppies have larger testes with greater sperm stores (Skinner and Watt 2007) and greater burst acceleration and swimming speed (Odell et al. 2003) than smaller males, which might facilitate the pursuit and sneak copulation of females and escape from predators.

Contrary to my findings, Schwartz and Hendry (2007) did not detect any female mating preference for any measured male trait, including male body size and orange and black ornamentation, in the same Trinidadian guppy population as ours (Upper Aripo River, Naranjo tributary). Differences in results between their study and mine might be attributable to differences in experimental design, measure of mating preference and sample size. Schwartz and Hendry used a no-choice design where females were allowed to physically interact with males, which permits the use of both visual and chemical cues in mate assessment, whereas test females in the current study (using a dichotomouschoice design) were only able to view the stimulus males and were unable to use chemical cues. Doughtery and Shuker (2015) suggest that choice tests elicit stronger mate preferences than do no-choice tests for two reasons. First, females may be better able to compare between males when they are presented simultaneously, as is the case in dichotomous-choice tests, compared to when males are presented sequentially, as in nochoice tests. Second, there is a greater cost associated with rejecting one option without being certain that another option is available, as would more likely be the case in nochoice tests. Choice tests, as used in my current study, are ecologically relevant for the guppy because females can assess multiple males concurrently as the sexes generally co- 
occur in mixed-sex shoals (Houde 1997; Auge et al. 2016) and females often encounter, and are pursued sexually by, two or more males simultaneously in nature (Jeswiet et al. 2011b). Additionally, Schwartz and Hendry (2007) scored female mating preference using their behavioural responses to male courtship displays (i.e. gliding, successful copulation), whereas I used the proportion of time that a female spent in the preference zone of each stimulus male as a proxy of her preference for that male. The latter proxy measure is known to be another reliable predictor of mate choice in female guppies (Dugatkin and Godin 1992; Kodric-Brown 1993). Lastly, sample size in the Schwartz and Hendry (2007) study was much smaller than in the current study and thus low statistical power might also explain their negative results (i.e. no female mating preference for any male trait).

Despite originating from a low-predation population where females are expected to exhibit strong preferences for orange in particular (Houde and Endler 1990; Endler and Houde 1995), females in my current study surprisingly did not exhibit as strong a preference as expected for males with relatively more orange or black pigmentation on their bodies. Weaker (but nonetheless significant) female mating preference for more ornamented males in my study population might be owing to relatively low amounts of orange and black ornamentation in wild males in this population (Auge et al. 2016; see also Tables 4-1 and 4-2) relative to other low-predation guppy populations in Trinidad (e.g. Houde and Endler 1990; Endler and Houde 1995; Houde 1997), which could make it more difficult for females to reliably discriminate amongst males based on their body colour patterns. Alternatively, Magurran and Seghers (1994) and Magurran (1998) have argued that female guppies in low-order, less productive streams in Trinidad (typically 
low-predation populations) need to spend more time foraging and it does not pay them to be as choosy in their choice of mates compared to females in high-order (typically highpredation populations) streams; such weaker choosiness could have constrained sexual selection for increasingly more ornamented males in the low-predation Upper Aripo (Naranjo tributary) population. Indeed, the extent of orange ornamentation in males within a population generally covaries positively with the strength of female preference for orange colour among Trinidadian guppy populations (Houde and Endler 1990; but see Houde and Hankes (1997) for an exception). However, the above Magurran and Seghers argument of reduced female mate choosiness in low-predation populations is not supported by the strong female mating preference for larger males in Upper Aripo River guppies which I report here.

In the current study, I purposefully used virgin females, as they are strongly receptive to male sexual advances (Houde 1997). Because more sexually experienced female guppies tend to be choosier than their less experienced counterparts (Pitcher et al. 2003), it is possible that non-virgin females would have demonstrated a stronger preference for males based on their ornamentation and(or) a stronger preference for larger males. My experimental design did not test for an interaction between male body size and male body ornamentation on female mating preferences. Other possible sources of variation in female mating preferences in the guppy include prior exposure to male phenotypes (Rosenqvist and Houde 1997; Pitcher et al. 2003), social familiarity (Hughes et al. 1999; Kelley et al. 1999; Zajitschek et al. 2006), and parasitism (Houde and Torio 1992). It is unlikely that prior exposure to male phenotypes, social familiarity or parasitism would have affected my results. All females in my experiment had prior visual 
exposure to males from their population. Males and females were reared in separated tanks on different laboratory shelves and thus would not have been familiar with each other prior to testing. All parental source fish were treated with anti-ectoparasite medication (LifeBearer ${ }^{\circledR}$ ) upon collection in the field and subsequently they and their offspring showed no signs of ectoparasitic infection in the laboratory.

All females used in my experiment were similar in health and prior social experience, but varied in standard length. Body length is generally correlated with age (Kodric-Brown and Nicoletto 2001a) and is a predictor of fecundity (Herdman et al. 2004) and thus overall female mate quality in the guppy. Cotton et al. (2006) suggested that female preference is likely to vary with female quality; my results support this proposition. More specifically, large non-gravid females, who are presumably of higher reproductive value to males than smaller females (Herdman et al. 2004), showed a stronger preference for larger males than did smaller females in the current study, but did not show a similar increase in their strength of preference for more ornamented males. Because male guppies prefer larger females as mates (Dosen and Montgomery 2004; Herdman et al. 2004; Jeswiet and Godin 2011; Jeswiet et al. 2012; Chapter 4), smaller females may gain less of a benefit from discriminating strongly between males based on body size compared with larger females. This discrepancy in benefit potentially accrued from discriminating between males based on body size can select against preferences for larger males in small females, which in turn can lead to the evolution of assortative mating (Cotton et al. 2006).

The absence of a relationship between female body length and female preference for more ornamented males in the current study could be the result of a weak preference 
for ornamented males and(or) little benefit to females in mating with more ornamented males (Pilastro et al. 2008) compared to the benefits gained by mating with larger males (Reynolds and Gross 1992; Watt et al. 2001; Cotton et al. 2006), irrespective of body ornamentation, in my study population. In comparison, the observed positive relationship between female body length and strength of mating preference for larger males may be owing to a positive covariation between body length and body condition in females, such that larger females are capable of exhibiting greater mating effort than smaller females (Cotton et al. 2006). However, contrary to this proposition, female guppies in the current study tended to spend less time associating with either stimulus male with increasing body length when given a choice between two stimulus males that differed in body size. My finding of a significant positive relationship between the body length of focal females and the strength of their mating preference based on male body length therefore cannot be explained by any increase in their sexual motivation or overall mating effort with increasing body length, as would also be predicted by life history theory (Stearns 1992). Alternatively, this observed increase in mating preference strength based on male body size with increasing body length could be linked to proximal changes in either the visual system and(or) cognitive abilities (Burns and Rodd 2008; Kotrschal et al. 2013) of female guppies with increasing age and associated body length.

In conclusion, I showed that laboratory-born virgin female guppies originating from a low-predation population in Trinidad exhibited inherent mating preferences for males based independently on their body length and colour ornamentation. I report for the first time, to my knowledge, that the mating preference of female guppies based on male body size was unexpectedly much stronger than that based on male ornamentation, and 
that larger females expressed stronger mating preferences for larger males than did smaller females, which could potentially lead to size-assortative mating in nature and important consequences for sexual selection and evolution (Jiang et al. 2013). My results thus contribute further to the understanding of both intra- and inter-population variation in mating preferences in the Trinidadian guppy, a long-standing important model species for the study of sexual selection and evolution. The current study should encourage further investigations into size-assortative mating and potential interactions between male body length and ornamentation on female mate choice in this model species and others.

\subsection{Acknowledgments}

I thank A.-C. Auge, D. Chowdhury and A.M. Miller for assistance with fish care, and two anonymous reviewers for constructive comments on the manuscript. 


\section{Chapter 4:}

\section{Covariation and Repeatability of Male Mating Effort and Mating Preferences in a Promiscuous Fish}

This chapter is published as: Godin, J.-G.J. and Auld, H. L. 2013. Covariation and repeatability of male mating effort and mating preferences in a promiscuous fish. Ecology and Evolution, 3: 2020-2029. It is reproduced here with permission from WileyBlackwell Publishing ${ }^{\mathcal{O}}$. 


\subsection{Abstract}

Although mate choice by males occurs in nature, our understanding of its importance in driving evolutionary change remains limited compared with that for female mate choice. Recent theoretical models have shown that the evolution of male mate choice is more likely to occur when individual variation in male mating effort and mating preferences exist and positively covary within populations. However, relatively little is known about the nature of such variation and its maintenance within natural populations. Here, using the Trinidadian guppy (Poecilia reticulata) as a model study system, I report that mating effort and mating preferences in males, based on female body length (a strong correlate of fecundity), positively covary and are significantly variable between subjects. Individual males are thus consistent, but not unanimous, in their mate choice. Both individual mating effort (including courtship effort) and mating preference were significantly repeatable. These novel findings support the assumptions and predictions of recent evolutionary models of male mate choice, and are consistent with the presence of additive genetic variation for male mate choice based on female size in my study population and thus with the opportunity for selection and further evolution of large female body size through male mate choice. 


\subsection{Introduction}

An individual's mate choice results from the interaction between its mating effort (investments in the sexual pursuit and attraction of prospective mates and in mating) and mating preference (differential ranking or choosing of prospective mates; Jennions and Petrie 1997; Edward and Chapman 2011). More specifically, increased investment in mating effort by a male increases his ability to attract multiple females, but consequently reduces his capacity to mate with those females, thus selecting for male mate choice (Edward and Chapman 2011). Males may therefore exert pre-mating choice by allocating more mating effort (including courtship) towards, and by accepting or rejecting, certain females over others (Edward and Chapman 2011).

Because of fluctuating selection and varying benefits and costs of mating behaviour in different environments, phenotypic plasticity in mating effort and mating preference are expected to be common and potentially beneficial for males (Qvarnström, 2001; Bretman et al. 2011a; Edward and Chapman 2011). If any male experiences greater competition for mates as a consequence of his preferring the same females as other males in the population, then males with either weak or alternative preferences will be at a sexually competitive advantage (Servedio and Lande 2006). Such male-male competition does not favour the evolution of a single shared male preference, but rather selects for individual variation in mating preferences (Servedio and Lande 2006; Edward and Chapman 2011). Evolution of mate choice is therefore more likely when individual variation in mating effort and mating preferences exist within populations (Servedio and Lande 2006; Rowell and Servedio 2009; Edward and Chapman 2011; South et al. 2012). Such variation can have major consequences for sexual selection and is of fundamental 
importance to the evolution of mate choice (Jennions and Petrie 1997; Widemo and Sæther 1999; Servedio and Lande 2006; Rowell and Servedio 2009; Edward and Chapman 2011; South et al. 2012). Therefore, understanding variation in mating effort and preference is critical for understanding both sexual selection and how diversity arises in nature (Jennions and Petrie 1997; Widemo and Sæther 1999). Within-population variation in female mating preferences has been documented to some extent, and its implications for the evolution of choice in females and elaborate sexual traits in males are widely recognized (e.g. Andersson 1994; Bakker and Pomiankowski 1995; Jennions and Petrie 1997; Widemo and Sæther 1999; Clutton-Brock 2007). This is not yet the case for male mate choice, but see Bell-Venner et al. (2008) for example.

A commonly-used measure of the variance structure of any phenotypic trait in a population is its repeatability, which represents the proportion of the total variation in the trait that can be attributable to differences between individuals (Boake 1989; Widemo and Sæther 1999). Repeatability is a measure of the within-individual consistency of the trait over time and is obtained from repeated measures on the same individuals. In quantitative genetics, repeatability of a trait is often used as an upper-bound estimate of its broad-sense heritability (i.e. fraction of total phenotypic variance that is genetic in basis) and thus its responsiveness to selection (Lynch and Walsh 1998). Notwithstanding its limitations (Jennions and Petrie 1997; Widemo and Sæther 1999; Dohm 2002), measuring the repeatability of mating preferences is a first step towards understanding how much preferences vary within a population (Widemo and Sæther 1999). Relatively little information is available on the repeatability of mating preferences expressed by 
males (Bell et al. 2009) and almost nothing is known about their heritability (Bakker and Pomiankowski 1995; Jennions and Petrie 1997; Chenoweth and Blows 2006).

To improve our relatively limited understanding of variation in male mate choice within natural populations, I characterize here both within- and between-subject variation in mating effort and mating preference directed towards females based on body size, and provide repeatability estimates for them, in wild-caught male Trinidadian guppies (Poecilia reticulata). Because a positive relationship between mating effort and mating preference for a particular female trait among males (Servedio and Lande 2006; Rowell and Servedio 2009; Edward and Chapman 2011), and female preference for males exhibiting high courtship effort (South et al. 2012), can in theory favour the evolution of male mate choice, I additionally tested for this relationship in male guppies. I used female body length as the target trait for male choice because it is a reliable proxy of female quality (highly correlated with fertility or fecundity) in the guppy (Reznick and Endler 1982; Kelly et al. 1999; Herdman et al. 2004; Ojanguren and Magurran 2004) and in many other species (Clutton-Brock 1988; Edward and Chapman 2011).

\subsection{Methods}

\subsubsection{Study animals}

I used wild-caught Trinidadian guppies as a study species. Paired stimulus females used in the experiment described below were gravid and matched as closely as possible for their degree of abdominal distension (assessed by eye), an indicator of stage of pregnancy (Houde 1997); see Chapter 2 for rationale. 


\subsubsection{General protocol}

Please see Chapter 2 for a description of the common general experimental protocol.

\subsubsection{Experimental apparatus}

The open-field test apparatus consisted of a glass aquarium (40 x $20 \times 25 \mathrm{~cm}$; L x W x H). See Chapter 2 for more information on test aquaria conditions.

\subsubsection{Experimental protocol}

To test for their mating effort and mating preference, I presented focal males with a simultaneous choice between two free-swimming stimulus females that were gravid, sexually non-receptive and differing in body length, with which they could interact physically, in an open-field apparatus to reflect the natural mate-encounter conditions experienced by wild Trinidadian guppies (cf. Houde 1997; Guevara-Fiore et al. 2010b; Jeswiet et al. 2011a). Both the test male and the stimulus females had full access to each other and thus to multiple stimuli (visual, chemical, tactile). With full access to sensory information, I assumed that males would to be able to accurately assess the differences in body size and reproductive state of the paired females (cf. Herdman et al. 2004; Hoysak and Godin 2007; Guevara-Fiore et al. 2010a,b).

I repeatedly recorded the mating effort and mating preference of individual focal males for either of two stimulus females differing in body length on each of two consecutive days (i.e. paired trials 1 and 2), with the repeated trials $23.5-24.5 \mathrm{~h}$ (hereafter, 1 day) apart, as follows. Observations on the focal male in trial 2 were made blind of his behaviour in trial 1 . On the day of a trial, two gravid females were matched 
by eye as closely as possible for abdominal distension (and thus reproductive state; Houde 1987) and measured for body length without anesthesia (chosen to be different in length) using a metric scale. Each focal male was presented with different stimulus females in the paired repeated trials to avoid the possibility of male recognition of a particular female. The body lengths of the stimulus females used in trials 1 and $2(N=80$, respectively) were very similar on average (Table 4-1). In any given trial, the paired stimulus females were chosen to similarly differ in body length on average by $4.8 \mathrm{~mm}$ (= $23.7 \%)$ in trial 1 and $4.8 \mathrm{~mm}(=24.0 \%)$ in trial $2\left(t\right.$-test, $t_{78}=0.206, P=0.837$; Table 41), to facilitate male mate choice based on female body size (cf. Dosen and Montgomerie 2004a; Herdman et al. 2004; Jeswiet and Godin 2011). 
Table 4-1 Mean \pm SE (range) standard body lengths of the focal males and paired stimulus females used on repeated consecutive days (paired trials 1 and 2), and the mean \pm SE absolute and relative differences in the standard body length of the paired stimulus females used in individual behavioural trial. Individual focal males were tested repeatedly in paired trials 1 and 2 .

\begin{tabular}{cccccc}
\hline \multirow{2}{*}{$\begin{array}{c}\text { Paired } \\
\text { trials }\end{array}$} & $\begin{array}{c}\text { Focal male } \\
(\mathrm{mm})\end{array}$ & $\begin{array}{c}\text { Larger female } \\
(\mathrm{mm})\end{array}$ & $\begin{array}{c}\text { Smaller female } \\
(\mathrm{mm})\end{array}$ & \multicolumn{2}{c}{$\begin{array}{c}\text { Difference between paired } \\
\text { females }\end{array}$} \\
\hline 1 & & & & $\mathrm{~mm}$ & $\%$ \\
& $18.4 \pm 0.3$ & $25.2 \pm 0.3$ & $20.4 \pm 0.3$ & $4.8 \pm 0.1$ & $23.7 \pm 0.9$ \\
& $(17.1-21.0)$ & $(22.0-30.0)$ & $(18.0-24.5)$ & $(3.0-6.5)$ & $(13.0-35.1)$ \\
2 & & & & & \\
& Ditto & $25.1 \pm 0.3$ & $20.3 \pm 0.3$ & $4.8 \pm 0.1$ & $24.0 \pm 0.9$ \\
& & $(22.5-30.5)$ & $(17.5-25.5)$ & $(2.5-7.5)$ & $(11.1-42.9)$ \\
\hline
\end{tabular}


Prior to the onset of a trial, the test aquarium was temporarily divided in half with a clear, perforated plastic partition. A focal test male was placed on one side of this partition and two stimulus females differing in body length were placed on the other side. All fish were left undisturbed for 30 min to allow them to acclimatize to the aquarium and to view and smell each other across the partition. After this period, the partition was raised and the behaviour of the fish was recorded live for $20 \mathrm{~min}$. I changed the water in the test aquarium with fresh aged water after every completed trial. I similarly tested a total of 40 males individually.

Following Herdman et al. (2004) and Jeswiet and Godin (2011), I recorded the following male sexual behaviours (cf. Houde 1997) directed towards either stimulus female during each of the paired trials: $(i)$ "approach", an unambiguous directed movement of the male towards a female, (ii) "association time", time during which a male actively follows within three body lengths a female with his head oriented towards her, (iii) "gonopodial nip", mouth-nipping behaviour by a male directed at a female's gonopore, $(i v)$ "sigmoid display", a courtship display directed at a female, which involves the male arching his body into an S-shape and quivering, and (v) "copulation attempt", scored as a male approaching a female from the side or behind and rapidly thrusting his gonopodium forward towards her genital opening. At the end of each pair of repeated trials, the standard body length of the focal male was measured (Table 4-1), and the focal male and stimulus females were placed in separate holding aquaria and not re-used.

I quantified the mating preference of each focal male in a given trial as the percentage of total association time spent near the larger female $(=[$ association time with large female / sum of association times with small and large females] x 100), and male 
mating effort as the percentage of total sexual acts (excluding association time) directed at the larger female. Male association time with a particular female is a reliable predictor of mate choice in the guppy (Jeswiet and Godin 2011), as well as for other fishes (e.g. Walling et al. 2010). Because each of the sexual acts measured potentially contributes to male mating success in guppies (Houde 1997), they thus collectively represent a male's pre-copulatory mating effort (cf. Edward and Chapman 2011). Additionally, a male was categorically classified as "preferring" a particular female if he spent $>50 \%$ of his total association time near her and concurrently directed $>50 \%$ of his sexual acts (mating effort) towards her in a given trial (cf. Godin and Dugatkin 1995; Jeswiet et al. 2011a, 2012).

\subsubsection{Statistical analysis}

All statistical analyses were carried out in the $R$ statistical software environment $(R$ Development Core Team 2012) and all tests are two-tailed, unless specified otherwise. Not all data were normal in distribution. Therefore, to improve normality and homoscedasticity, percentage (proportion) data were arcsine transformed and data on counts were $\log _{10}$ transformed prior to analysis. The two main dependent behavioural variables of interest here (i.e. male mating effort, male mating preference) were normally distributed (Shapiro tests, all $P>0.492$ ) and homoscedastic (Levene tests, all $P>0.298$ ) following transformation.

I first tested the null hypothesis of no difference in the mating preference and mating effort of focal males for either of the paired stimulus females by comparing separately their mating preference (percent of association time with the larger female) 
and mating effort (percent of total sexual acts directed towards the larger female) scores, and the number of males categorized as preferring the larger female, against that expected by chance using the Wilcoxon signed-rank test and Binomial test, respectively. I then separately compared the frequencies of each of the recorded sexual acts directed by males towards the larger and smaller females using the Wilcoxon signed-rank test. I used linear mixed-effects models (LMMs) with the restricted maximum-likelihood (REML) method to test for any effects of trial number (trial 1 vs. trial 2), male body length and difference in the length of paired stimulus females on the mating preference and mating effort scores of focal males separately, controlling for male identity as a random variable in the models (Crawley 2007; Field et al. 2012).

Second, to characterize the relationship between male mating preference and mating effort, I correlated $(i)$ the mating preference scores of individual males against their total mating effort (= total sexual acts) scores and (ii) mating preference scores against courtship effort scores (= percent of courtship displays exhibited towards the larger female) for each of the paired repeated trials separately using the Spearman rank correlation analysis. For this analysis, I necessarily excluded from the data set those individual behavioural trials ( $N=9$ out of 80 trials) in which the focal male did not court either stimulus females, but otherwise exhibited all the other sexual acts towards them.

Lastly, I separately calculated the repeatability of male mating preference and mating effort scores between paired trials 1 and 2 using linear mixed-effects models (LMMs) with the restricted maximum-likelihood (REML) method in the 'rptR' package ('rpt.remlLMM' function; Nakagawa and Schielzeth 2010) developed in R software (R Development Core Team 2012), given that the data were Gaussian. In the models, fish 
identity was assigned as a grouping random factor (Nakagawa and Schielzeth 2010). For each estimated repeatability $(R)$ coefficient, I provided the associated calculated standard error (SE) and 95\% confidence interval (CI). To ascertain whether the mating preference or mating effort score of a focal male in trial 1 would predict his preference or mating effort score, respectively, one day later in trial 2, I regressed separately (using simple linear regression analysis) each of these two behavioural measures obtained for trial 1 against that for paired trial 2 .

\subsubsection{Ethical standards}

This study was approved by the institutional Animal Care Committee at Carleton University (protocol\# B10-33), and thus adheres to the animal use and care guidelines of the Canadian Council on Animal Care and the laws of Canada.

\subsection{Results}

All focal males used were sexually active and directed mating effort towards both paired stimulus females. As expected, the stimulus females used did not exhibit any obvious sexual behaviour towards the focal males in the current study (because they were gravid and thus unreceptive to male sexual solicitations; cf. Houde 1997).

On average, males exhibited significantly greater mating effort towards, and spent more time associating with, the larger female than expected by chance in both paired trials (Wilcoxon signed-rank test, all $P<0.0001$, Figure $4-1$ ). Therefore, significantly more males than expected by chance ( 33 out of 40 in Trial 1, and 32 of 40 in Trial 2) categorically preferred the larger of the two stimulus females (one-tailed Binomial test, 
both $P<0.0001)$. Considering the constituent components of mating effort, males exhibited significantly more approaches, sigmoid courtship displays, gonopodial nips and copulation attempts towards the larger of the paired stimulus females (Wilcoxon signedrank test, all $P<0.0001$, Figure 4-2). 


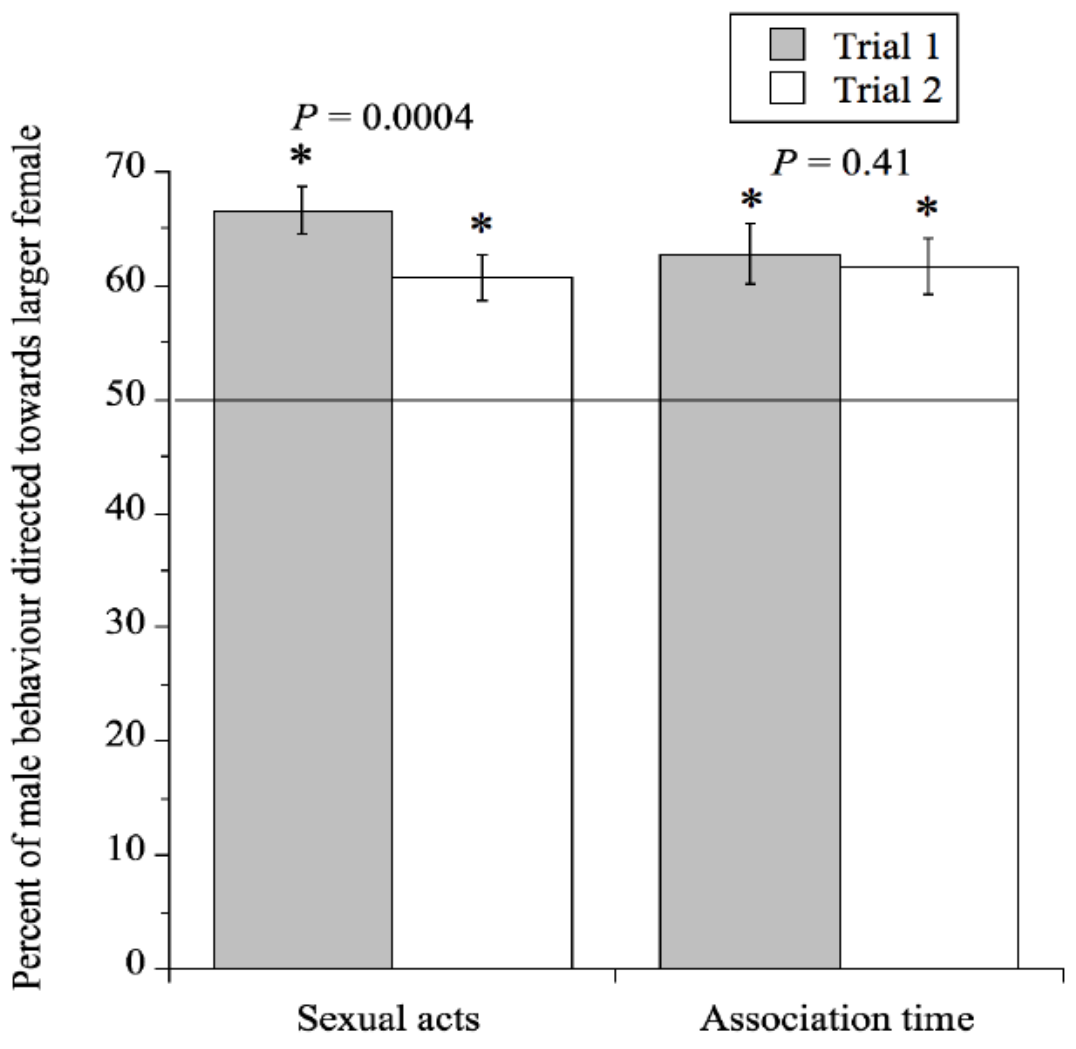

Figure 4-1 Mating effort and mating preference of focal males for the larger female in each of the repeated trials. Mating effort is depicted as the mean \pm SE percent of total sexual acts directed by focal males towards the larger female, and mating preference as the mean $\pm \mathrm{SE}$ percent time focal males spent associating with the larger female. The horizontal line denotes random choice between the paired stimulus females. The asterisk above individual histogram bars indicates a significant $(P<0.0001$; Wilcoxon signedrank test) difference between the observed mean score and that expected from chance (i.e. 50\%) for that particular trial. The $P$-value above each pair of histogram bars (paired trials 1 and 2) was obtained using LMM models testing for any effect of trial number on the behavioural scores of males in each experiment. 


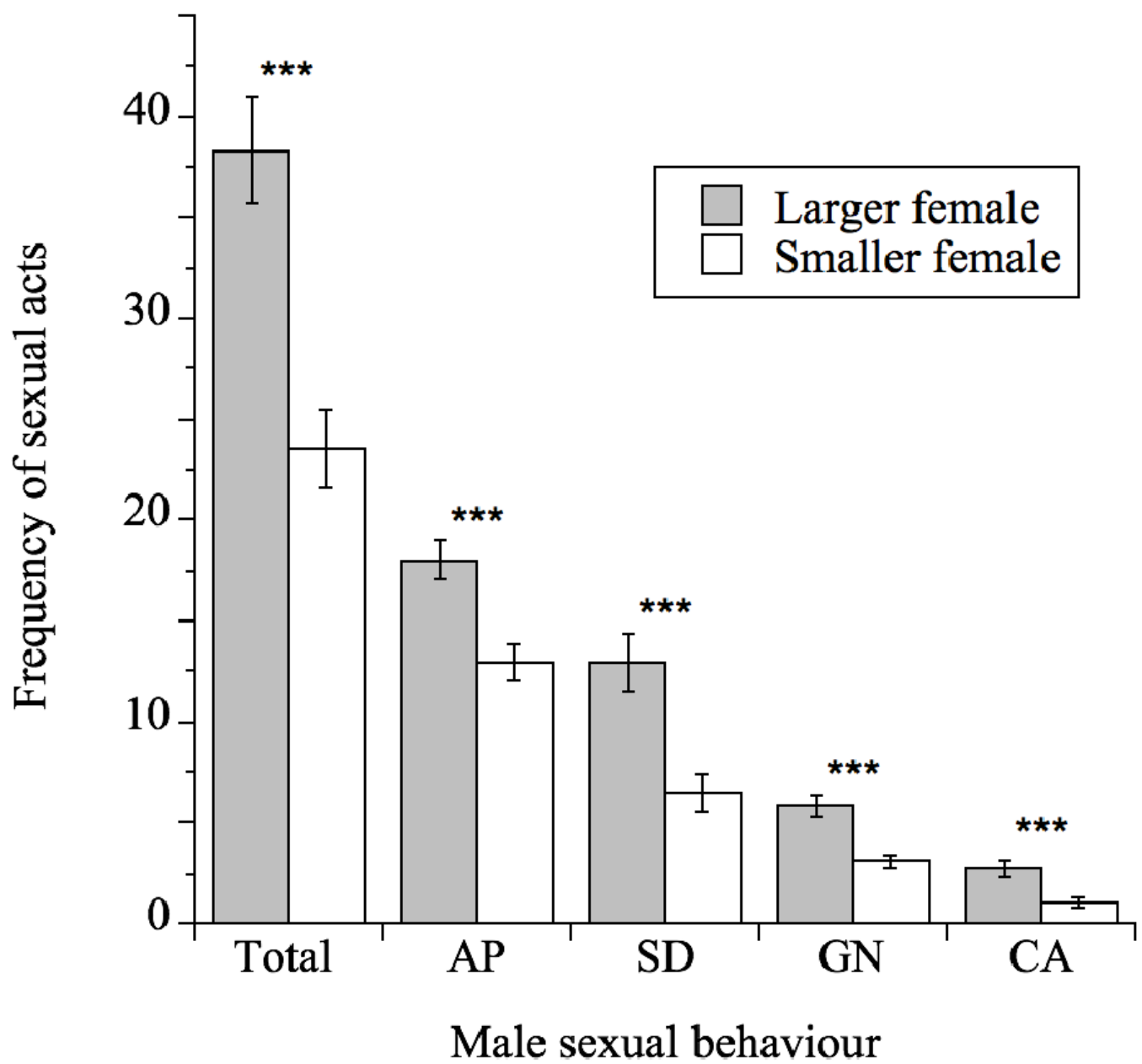

Figure 4-2 Male mating effort expressed as the mean \pm SE frequency (number/40 min) of each of the different component sexual acts, and their total sum, directed by focal males towards each of the paired stimulus females over the course of the repeated paired trials. $\mathrm{AP}=$ approach, $\mathrm{SD}=$ courtship sigmoid display, $\mathrm{GN}=$ gonopodial nip, $\mathrm{CA}=$ copulation attempt. $* * * P<0.0001$, obtained using the Wilcoxon signed-ranks test. 
Although males preferred larger females on average, the scores for two measured proxy components of their mate choice (mating preference and mating effort) varied widely among individuals and were significantly positively correlated with each other in both repeated trials (Figure 4-3A, B). Similarly, a significant positive relationship between mating preference and courtship effort (a component of mating effort) was also observed in both repeated trials (Figure 4-3C, D). Neither mating preference or mating effort scores of individual males were influenced by their body length (linear mixedeffects models (LMMs), $t_{38}=1.28, P=0.209 ; t_{38}=1.20, P=0.237$, respectively) or the difference in the body length of the paired stimulus females (LMMs, $t_{38}=1.05, P=$ $0.301 ; t_{38}=0.52, P=0.603$, respectively).

The mating effort and mating preference of individual males were also both highly repeatable between the paired trials, as measured by sexual acts directed towards the larger female (repeatability estimate, $R=0.628 \pm 0.099,95 \% \mathrm{CI}=0.403-0.786, P<$ 0.0001 ; Figure 4-4A) and by association time with the larger female $(R=0.824 \pm 0.053$, $95 \% \mathrm{CI}=0.703-0.905, P<0.0001$; Figure 4-4B), respectively. That is, there was significant variation in both mating effort and mating preference between subjects, and individual subjects were consistent in these sexual behaviours over time. 

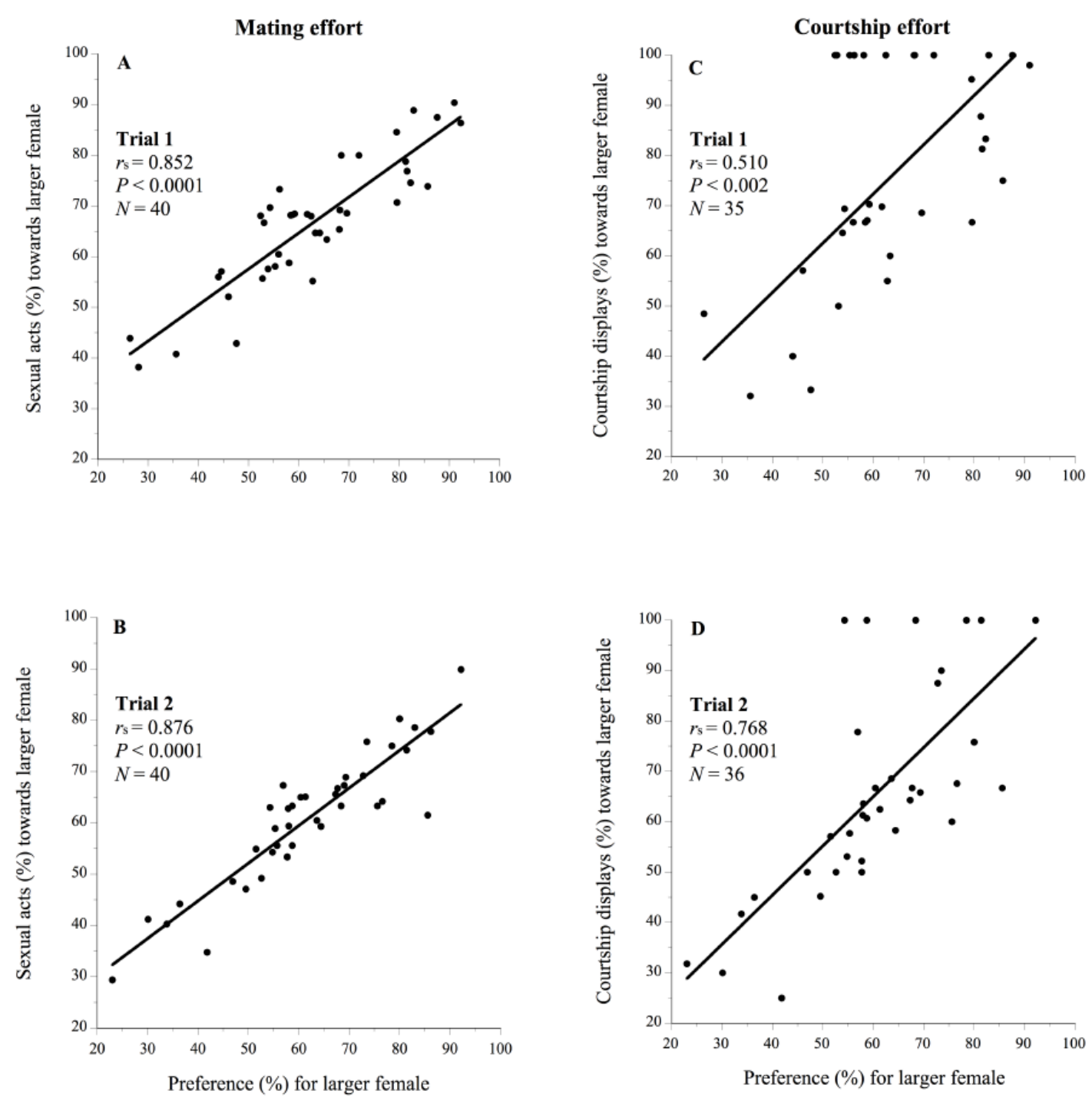

Figure 4-3 Relationships between the mating preference of focal males, based on percent association time with the larger female, and their mating effort (panels A and B) and courtship effort (panels $\mathrm{C}$ and D) for paired repeated trials 1 and 2 . The best-fit lines were obtained using simple linear regression analysis. The correlation coefficient $\left(r_{s}\right)$ and $P$ values shown were obtained using the Spearman rank correlation analysis. 

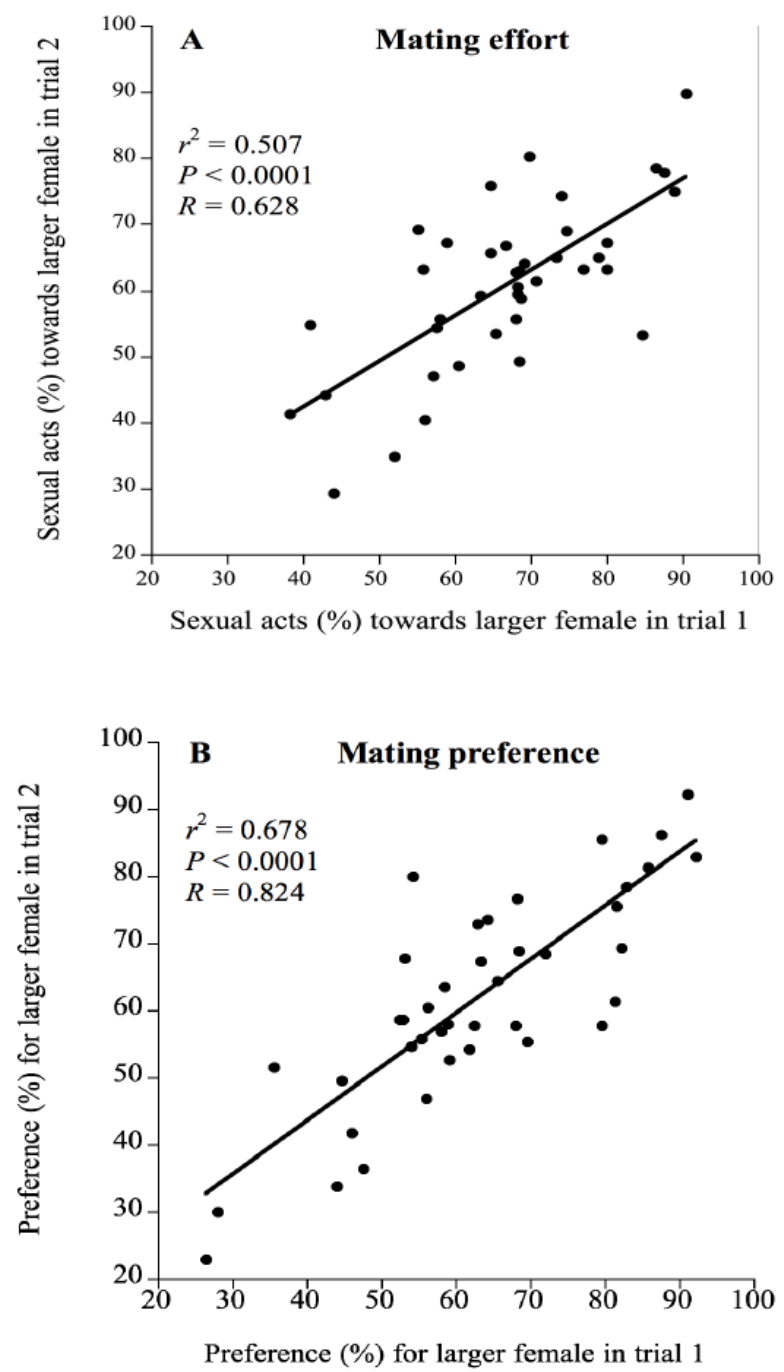

Figure 4-4 Relationships between the mating effort scores of focal males, based on the percent of sexual acts directed towards the larger female (panel A), and their mating preference scores, based on percent association time with the larger female (panel B), during trial 1 and their scores one day later in repeated trial 2. The best-fit lines, and associated $r^{2}$ and $P$ values, were obtained using simple linear regression analysis. Repeatability $(R)$ estimates were obtained using the linear mixed-effects model with the restricted maximum-likelihood (REML) method described in Nakagawa and Schielzeth (2010). 


\subsection{Discussion}

Although male mate choice does occur in nature, it is not as well understood as female mate choice (Andersson 1994; Amundsen 2000; Bonduriansky 2001; Clutton-Brock 2007; Edward and Chapman 2011). Because male mate choice is more likely to evolve when individual variation in mating effort and mating preference exists, and when male courtship effort (a component of mating effort) and mating preference positively covary (Servedio and Lande 2006; Rowell and Servedio 2009; Edward and Chapman 2011; South et al. 2012), understanding such phenotypic variation is critical for understanding sexual selection and the evolution of mate choice (Jennions and Petrie 1997; Widemo and Sæther 1999; Edward and Chapman 2011). In this context, the results of my current study support the assumptions and predictions of recent evolutionary models of male mate choice (Servedio and Lande 2006; Rowell and Servedio 2009; South et al. 2012) and thus advance our understanding of the importance of phenotypic variation (in mating effort and preference) within natural populations in the evolution of male mate choice.

Here, I showed that male Trinidadian guppies on average directed significantly greater mating effort and exhibited a preference for (as measured by association time with) the larger of two females, independent of female identity, when presented concurrently in an open-field arena. The observed preferences cannot be explained by any differential sexual behaviour of the (gravid) stimulus females toward focal males, as they were generally sexually unreceptive to males (cf. Houde 1997), nor by variation in male body length or body length difference between paired stimulus females. These results generally corroborate those of Abrahams (1993), Dosen and Montgomerie (2004a), Herdman et al. (2004), Head et al. (2010) and Jeswiet et al. (2012), on guppies 
of different provenances than mine, and thus collectively demonstrate that male guppies possess a generalized mating preference for large females. Male allocation of greater mating effort towards, and preference for, larger and more fecund females as mates is fairly widespread taxonomically (Andersson 1994; Bonduriansky 2001; Clutton-Brock 2007; Edward and Chapman 2011). Preferring to mate with large females appears to be adaptive, as large female guppies are more fecund (Reznick and Endler 1982; Kelly et al. 1999; Herdman et al. 2004; Ojanguren and Magurran 2004) and thus of potentially greater reproductive value to males than smaller ones (Parker 1983; Andersson 1994; Edward and Chapman 2012), all else being equal.

However, if all males have an equal amount of resources that they can allocate to courtship (or mating effort in general) and similarly bias the distribution of this effort towards preferred females in polygynous or promiscuous systems, then male choice expressed as increased courtship towards preferred females can lead to increased male mating competition for the most attractive females (and potentially increased sperm competition, Wedell et al. 2002) in the population and consequently to a loss of a male preference allele (Servedio and Lande 2006). This cost of increased competition for preferred females, which constrains the evolution of male mate choice, can be offset or mitigated if $(i)$ preferred females have sufficiently higher fecundity or mating success (Parker 1983; Servedio and Lande 2006; Edward and Chapman 2012), (ii) males can avoid or minimize sperm competition by adjusting their mating effort and preference accordingly (Wedell et al. 2002; Rowell and Servedio 2009), and(or) (iii) courtship is costly and males differ in how they distribute their courtship effort among females and 
females prefer males that exhibit high courtship effort (Servedio and Lande 2006; Rowell and Servedio 2009; South et al. 2012).

The latter strategic conditions favouring the evolution and maintenance of male mating preferences in populations exist in the guppy mating system. More specifically, male guppies exhibit on average a mating preference for larger females (current study; Abrahams 1993; Herdman et al. 2004; Head et al. 2010; Ojanguren and Magurran 2004; Jeswiet et al. 2011a), female fecundity is strongly positively correlated with their body size (Reznick and Endler 1982; Kelly et al. 1999; Herdman et al. 2004; Ojanguren and Magurran 2004), and larger multiply-mated females produce larger (and presumably more viable) offspring than smaller females (Ojanguren et al. 2005). Male courtship behaviour is costly (Godin 1995; Head et al. 2010) and an honest, condition-dependent indicator of male quality (Nicoletto 1993; Houde 1997; Matthews et al. 1997; Kolluru et al. 2009), and females prefer males exhibiting with high courtship effort, at least in some populations (Houde 1997; Kodric-Brown and Nicoletto 2001b). Moreover, male guppies are sensitive to the local risk of sperm competition and adaptively reduce their mating preferences for larger, more fecund females and re-direct their mating effort towards smaller, less fecund females in response to a perceived increase in the risk of sperm competition associated with larger, more attractive females (Jeswiet et al. 2011b, 2012), thereby potentially contributing to the maintenance of variation in male mating preferences in the population. Lastly, as I demonstrated in the current study, males vary widely and consistently in their mating effort (including courtship) and mating preference for females based on body size, and individual male mating effort (including courtship effort) and mating preferences are strongly, positively correlated. 
Phenotypic and genetic variation in traits is required for selection (Lynch and Walsh 1998), and variation owing to individual plasticity in mating behaviour may be adaptive under a range of conditions (Qvarnström 2001; Bretman et al. 2011a). Although other investigators have previously observed variation among male guppies in their mating preferences based on female body size (Abrahams 1993; Dosen and Montgomerie 2004a; Jeswiet and Godin 2011; Jeswiet et al. 2011a, 2012), this current study is the first to comprehensively characterize and analyze individual variation in both male mating effort and mating preference and to report on their repeatabilities for any species (cf. Bell et al. 2009) to my knowledge. I showed that mating effort and mating preference in wildcaught male guppies positively covary and are significantly more variable among than within individuals, and that individual males are thus consistent, but not unanimous, in their mate choice (cf. Widemo and Sæther 1999), at least in my study population. The nature of such phenotypic variation in mating effort and preference would maintain male mate choice, once evolved, within the population (cf. Servedio and Lande 1996; Edward and Chapman 2011). To the extent that repeatability of a trait places an upper limit on its heritability (Lynch and Walsh 1998), the observed high repeatabilities for mating effort and mating preference, based on female body size, obtained for Upper Aripo River male guppies here is consistent with the presence of additive genetic variation for both these traits in this population and thus with the opportunity for selection and further evolution of large female body size through male mate choice. There is additionally some limited evidence for a genetic basis to male courtship effort in the guppy (Nicoletto 1995; Mariette et al. 2006). However, the evolutionary exaggeration of female body size in the guppy, under directional male mate choice, is constrained by resource limitation, life- 
history trade-offs and costs associated with large body size in females (Magurran 2005) and by sperm competition (Kelly et al. 1999; Neff et al. 2008; Dosen and Montgomerie 2004b; Jeswiet et al. 2011b, 2012) and polymorphism in the allocation of mating effort and mating preference by males among females in the population (current study).

Given the relatively limited number of studies on variation in male mate choice (Bell et al. 2009; Edward and Chapman 2011), my current study thus represents an important contribution to further characterizing and understanding variation in male mate choice and its evolution within natural populations. Enduring challenges include understanding the genetic and environmental bases of individual variation in male mating effort and mating preference, the relationship between individual variation in mate choice and variance in lifetime reproductive success among males, and the interactions between male and female mate choice on sexual selection in species with mutual mate choice (Edward and Chapman 2011). Because it exhibits mutual mate choice, male reproductive skew (Neff et al. 2008) and within-population variation in repeatable mating preferences in both males (current study) and females (Godin and Dugatkin 1995), the Trinidadian guppy offers a particularly suitable model species for pursuing these lines of investigation in the future.

My novel main findings, of positive covariation and high repeatability in mating effort and mating preference in male guppies, are consistent with the assumptions and predictions of recent models for the evolution of male mate choice (Servedio and Lande 2006; Rowell and Servedio 2009; South et al. 2012) and suggest that such directional mating effort and preference in male guppies select for large body size in females, have a genetic basis, and are potentially responsive to selection and further evolution. 


\subsection{Acknowledgments}

I thank the Director of Fisheries, Ministry of Agriculture, Land and Marine Resources of Trinidad and Tobago for permission to collect guppies from the Upper Aripo River for use in my study, I.W. Ramnarine for providing logistical support at the University of the West Indies, St. Augustine, Trinidad, T.N. Sherratt for advice on statistical analyses, S.M. Bertram and D.T. Gwynne for constructive comments on the manuscript. 


\section{Chapter 5:}

\section{Male Guppies Use Social Information and Copy the Mate Choice of Sexual Rivals}

This chapter is published as: Auld, H.L. and Godin, J.-G.J. 2015. Sexual voyeurs and copiers: social copying and the audience effect on male mate choice in the guppy. Behavioral Ecology and Sociobiology, 69: 1795-1807. It is reproduced here with permission from Springer-Verlag Berlin Heidelberg ${ }^{\complement}$. 


\subsection{Abstract}

In most mating systems, males and females are commonly within signaling and receiving distance of conspecifics during courtship and mating activities. Although it is well known that females who observe sexual interactions between conspecifics will use public information obtained from these interactions when making their own mating decisions, much less is known about whether males use this type of information in making mating decisions. I used the Trinidadian guppy (Poecilia reticulata) to test whether males use public information to copy the apparent mate choice of another male. I show that males use public information to copy the mate choice of other males. These results indicate that males pay attention to their immediate social environment in making mating decisions. My findings highlight the importance of social context and public information in male mate-choice decisions and have implications for the evolution of male mating preferences and of social information use in populations. 


\subsection{Introduction}

In most mating systems, males and females are commonly within signal-receiving distances of each other, thus forming communication networks (McGregor and Peake 2000). In such networks, bystanding or eavesdropping male sexual competitors (the 'audience', McGregor and Peake 2000) can observe other nearby males court, or otherwise sexually interact, with females. When sexually interacting with a particular female, a male may inadvertently reveal his mating preference to nearby eavesdropping male rivals, thereby conveying to them public information (sensu Danchin et al. 2004) about the sexual attractiveness and(or) receptivity of that female. In situations where males face high mating effort costs (Svensson et al. 2004; Hoefler et al. 2008), it may be beneficial for a bystanding male to minimize his individual costs of locating, attracting and assessing sexual partners by using this less costly form of public information (McGregor and Peake 2000; Danchin et al. 2004) and "copy" the observed mating preference (for a given trait) or mate choice (for a specific partner) of another male (reviewed by Witte 2006a; Vakirtzis 2011). If an eavesdropping male copies the mate choice of another male, then both males will be mating with the same female and thereby incur a greater risk of sexual (Witte 2006a) and potentially sperm competition (Parker et al. 1997) than otherwise. However, this cost might be offset by the lower costs of finding and assessing mates for the copier. Mate-choice copying has been documented for females in numerous taxonomically diverse species, including humans (e.g., Dugatkin 1992; Dugatkin and Godin 1992; Galef and White 1998; Munger et al. 2004; Place et al. 2010; Yorzinski and Platt 2010). However, to my knowledge, empirical evidence for mate-choice copying by males is limited to only a handful of species, including the sex- 
role reversed pipefish (Syngnathus typhle, Widemo 2006), sailfin molly (Poecilia latipinna, Schlupp and Ryan 1997; Witte and Ryan 2002), Atlantic molly (Poecilia mexicana, Bierbach et al. 2011b), three-spined stickleback (Gasterosteus aculeatus, Frommen et al. 2009), and humans (Homo sapiens, Place et al. 2010; Yorzinski and Platt 2010).

The opportunity for male mate-choice copying has often been overlooked, as males generally have a much higher reproductive potential than females, are thought to be less discriminatory, and will incur lower fitness costs than females if they make a poor mate choice decision. Although male mate-choice copying seems counter-intuitive, as it potentially increases the risk of sperm competition, it may evolve if it minimizes lost mating opportunity costs. When there is a conflict in mating rates between males and females in a species, females may be unreceptive to male sexual advances, thereby limiting male mating opportunities. In a situation where a receptive mate is difficult to come by, males will incur high trial-and-error costs from sampling females. Public information from other males could reduce the trial and error costs of locating a receptive female. Increased sexual activity of other males may be indicative of female sexual receptivity, and in many species, female receptivity is the main limiting factor in male reproductive success (Andersson 1994). The high cost of mate sampling could lead to male mate-choice copying despite the potential for increasing sperm competition risk.

Here, using the Trinidadian guppy (Poecilia reticulata) as a model study system, I tested the hypothesis that males will use inexpensive social information and copy the choice of sexual rival when choosing a mate. 


\subsection{Methods}

\subsubsection{Study animals}

I used wild-caught Trinidadian guppies as a study species. Paired stimulus females used in the experiment described below were gravid and matched as closely as possible for their degree of abdominal distension (assessed by eye), an indicator of stage of pregnancy (Houde 1997); see Chapter 2 for rationale. However, they were chosen to differ in standard length (measured from tip of snout to caudal peduncle) by $19.3 \pm 0.9 \%$ (mean \pm SE; Table 5-1) to promote male discrimination between the females and to elicit a choice from the male (Dugatkin and Godin 1993; Jeswiet et al. 2011; Godin and Auld 2013; Chapter 4). All males used in any given behavioural trial were matched for standard length (measured with a metric scale) and colour ornamentation as closely as possible (assessed by eye, but later confirmed from analysis of their digital photograph; see Chapter 2 for methodology). 
Table 5-1 Mean \pm SE (range) standard body length $(\mathrm{mm})$ and body ornamentation score $(\%$ of male body covered by orange and black pigmentation) of guppies used in the two treatments of the mate-choice copying experiment. Results of ANOVA tests ( $F$-values) and $t$-tests for homogeneity of body length and colour score among male types, and results of paired $t$-tests comparing the body length of paired stimulus females within and between treatments, are shown.

\begin{tabular}{|c|c|c|c|c|c|c|c|c|}
\hline \multirow[b]{2}{*}{ Treatment } & \multicolumn{3}{|c|}{ Male length $(\mathrm{mm})$} & \multicolumn{3}{|c|}{ Male colour score $(\%)$} & \multicolumn{2}{|c|}{ Stimulus female length (mm) } \\
\hline & Focal & Model & Hidden model & Focal & Model & Hidden model & Large female & Small female \\
\hline $\begin{array}{l}\text { No copying } \\
\text { opportunity }\end{array}$ & $\begin{array}{c}19.1 \pm 0.3 \\
(17.3-21.4)\end{array}$ & - & - & $\begin{array}{c}6.4 \pm 0.5 \\
(2.5-9.3)\end{array}$ & - & - & $\begin{array}{c}26.7 \pm 0.3 \\
(24.2-30.5)\end{array}$ & $\begin{array}{c}22.3 \pm 0.4 \\
(19.0-25.7)\end{array}$ \\
\hline $\begin{array}{c}\text { Within } \\
\text { treatment } \\
\text { comparison }\end{array}$ & \multicolumn{3}{|c|}{-} & \multicolumn{3}{|c|}{ - } & \multicolumn{2}{|c|}{$t_{38}=-8.2, P<0.001$} \\
\hline $\begin{array}{c}\text { Copying } \\
\text { opportunity }\end{array}$ & $\begin{array}{c}18.8 \pm 0.2 \\
(17.4-20.5)\end{array}$ & $\begin{array}{c}18.6 \pm 0.2 \\
(17.3-20.1)\end{array}$ & $\begin{array}{c}18.9 \pm 0.2 \\
(17.5-20.4)\end{array}$ & $\begin{array}{c}6.5 \pm 0.5 \\
(1.6-11.0)\end{array}$ & $\begin{array}{c}5.4 \pm 0.5 \\
(1.2-9.6)\end{array}$ & $\begin{array}{c}6.2 \pm 0.6 \\
(1.8-10.3)\end{array}$ & $\begin{array}{c}27.5 \pm 0.5 \\
(23.1-31.7)\end{array}$ & $\begin{array}{c}23.2 \pm 0.4 \\
(19.8-25.8)\end{array}$ \\
\hline $\begin{array}{c}\text { Within } \\
\text { treatment } \\
\text { comparisons }\end{array}$ & \multicolumn{3}{|c|}{$F_{2,57}=0.41, P=0.66$} & \multicolumn{3}{|c|}{$F_{2,57}=0.97, P=0.39$} & \multicolumn{2}{|c|}{$t_{38}=-6.7, P<0.001$} \\
\hline $\begin{array}{c}\text { Across } \\
\text { treatment } \\
\text { comparisons }\end{array}$ & $\begin{aligned} t_{38} & =0.84 \\
P & =0.40\end{aligned}$ & - & - & $\begin{array}{c}t_{38}=-0.79 \\
P=0.94\end{array}$ & - & & $\begin{array}{l}t_{38}=1.2 \\
P=0.23\end{array}$ & $\begin{array}{l}t_{38}=1.7 \\
P=0.09\end{array}$ \\
\hline
\end{tabular}




\subsubsection{General protocol}

See Chapter 2 for a description of the common general protocol.

\subsubsection{Experimental apparatus}

The apparatus consisted of a clear Plexiglas test aquarium $(40 \times 20 \times 25 \mathrm{~cm}$; L x W x H; Figure 5-1) flanked by a clear Plexiglas container at either end (15 x $20 \times 25 \mathrm{~cm}$; Figure 5-1). The central aquarium served as the mate-choice arena and held the focal male, and the end containers (hereafter 'end compartments') each contained a stimulus female differing in body length. See Chapter 2 for more information on general test aquaria conditions.

\subsubsection{Experimental protocol}

This experiment comprised two treatments and generally followed the protocol used by Dugatkin and Godin (1992) for ascertaining the occurrence of mate-choice copying. The Copying treatment (Figure 5-1) provided individual focal males with the opportunity to copy the experimentally-staged mating preference of another male (the model), whereas no such opportunity was available in the Control treatment. Briefly, each experimental trial consisted of three consecutive 20-min phases (Figure 5-1): (i) an initial mating preference test (Preference Test 1) to establish the baseline preference of the focal male, (ii) a viewing period during which the focal male could observe the stimulus female that he did not prefer in Preference Test 1 either with no model male present near her (Control treatment: no copying opportunity) or with a visible model male exhibiting sexual interest in her (Copying treatment: copying opportunity present), and (iii) a second mating 

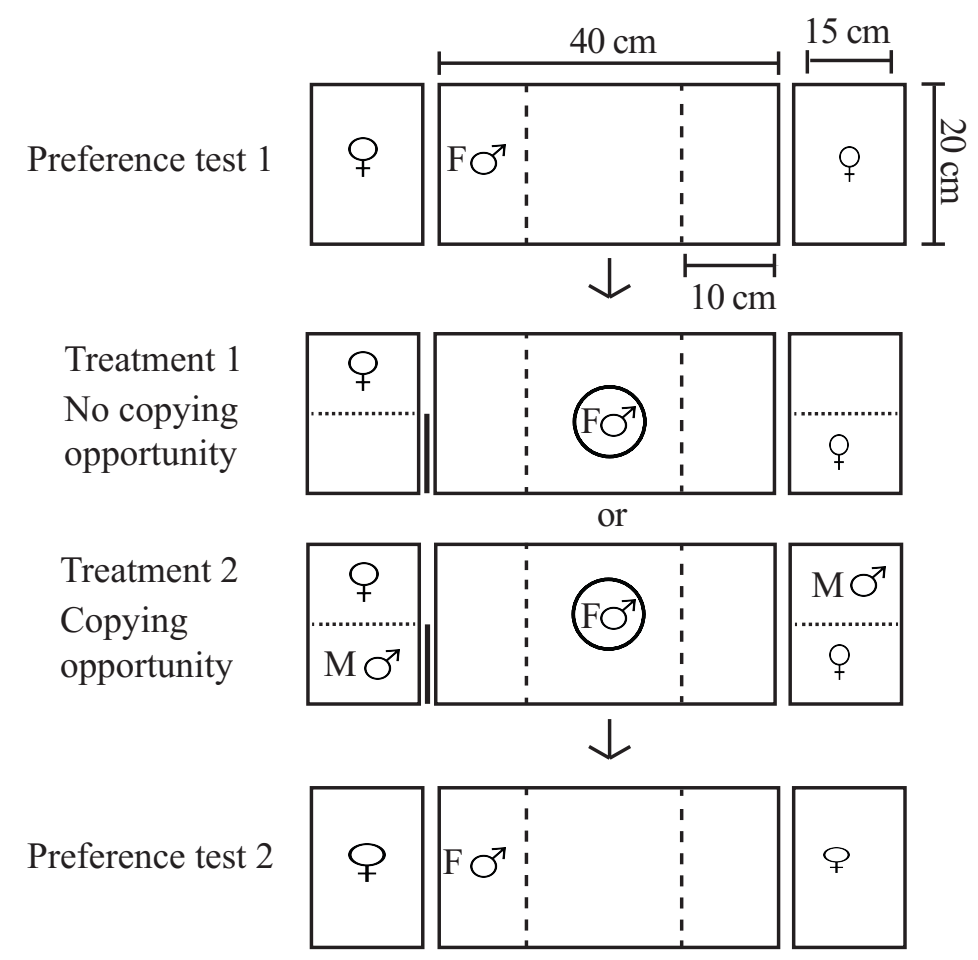

Figure 5-1 Schematic top view of the experimental apparatus used in the mate-choice copying experiment. The central compartment $(40 \times 20 \times 25 \mathrm{~cm} ; \mathrm{L} \times \mathrm{W} \times \mathrm{H})$ held the focal male (F) and the end compartments $(15 \times 20 \times 22 \mathrm{~cm})$ held the model males (M) and (or) stimulus females, depending on the treatment. The circle represents a removable clear Plexiglas cylinder ( $7 \mathrm{~cm}$ diam.), in which the focal male could temporarily be placed. The thick dark line in front of one of the end compartments denotes an opaque Plexiglas screen. Dotted lines represent removable clear Plexiglas partitions that allowed the end compartments to be either divided in half or left open. 
preference test (Preference Test 2) with no model male present. Therefore, each focal male was tested twice for his mating preference and thus acted as his own control. The Control treatment tested for consistency in male mating preference between the two consecutive preference tests, whereas the Copying treatment ascertained whether a focal male's initial mate choice could be altered by an opportunity to copy the apparent mating preference of another male (the model).

A mate-choice trial consisted of initially placing a focal male into a clear Plexiglas cylinder $(7 \mathrm{~cm}$ diam.) located in the centre of the test aquarium and randomly placing one stimulus female into each of the two end compartments. The focal male's initial (baseline) mating preference was ascertained in Preference Test 1 as follows. All fish were left undisturbed in their compartments for a 10-min acclimatization period before the start of the preference test, during which time opaque screens blocked the focal male's view of the stimulus females in the end compartments. After this period, I removed the aforementioned opaque screens allowing the focal male to observe the stimulus females. After 2 min I released the focal male from the Plexiglas cylinder and allowed him to 'choose' between the two stimulus females for $10 \mathrm{~min}$. Immediately following this choice period, I replaced the opaque screens and gently dip-netted the focal male to place him into the central cylinder. I then switched the positions of the two end compartments to control for any potential side bias in the male's preference behaviour (see Chapter 2 for details). I removed the screens, released the male from the cylinder, and scored his mating preference for a second 10-min period. Thus, the mating preference of the focal male was quantified over a total of 20 min (i.e. Preference Test 1). Immediately following Preference Test 1, I replaced the opaque screens and 
placed the focal male in the central cylinder. To start the second (viewing) phase of the trial, each of the two end compartments was temporarily divided in half parallel to the long axis of the test aquarium with a clear Plexiglas partition (Figure 5-1). For the Copying treatment, I introduced a model male next to the female that the focal male had initially not preferred in Preference Test 1; this model male was visible to the focal male held in the cylinder (Figure 5-1). The model male typically moved along the clear Plexiglas partition, whilst following the movements of the female on the other side, and exhibited courtship displays towards her. This staged encounter simulated an apparent (non contact) sexual interaction between the visible model male and the initially nonpreferred female, presumably demonstrating to the observer focal male that this particular female is attractive to another male. To minimize potential differences in the activity level and behaviour of the females (Schlupp et al. 1994; Patriquin Meldrum and Godin 1998; Witte and Ryan 1998), I similarly placed a model male next to the other (initially preferred) female; however, this model male was hidden from the view of the focal male by an opaque screen in front of its compartment (Figure 5-1). I alternated between trials whether the large female was placed in the left or right end compartment and the particular half of the compartments in which the visible and hidden models were placed. After I placed the model males in the end compartments, I removed the opaque screens and allowed the focal male (whilst still in the cylinder) to observe for 20 min the initially non-preferred female socially interact with a visible model male and the other female apparently alone. At the end of this viewing period, I replaced the opaque screens and gently removed the model males and Plexiglas partition from the end compartments (this procedure took less than $1.5 \mathrm{~min}$ ). I then removed the screens and released the focal 
male from the cylinder allowing him to choose between the two stimulus females for a second 20-min period (i.e. Preference Test 2), as for Preference Test 1. I used the same protocol for the Control treatment, except that I did not introduce a model male in either of the two end compartments.

For any preference test, the preferred stimulus female was considered the one with whom the focal male spent more than $50 \%$ of his total association time (summed over the paired females). This criterion, or slight variants of it, for mating preference is commonly used for poeciliid fishes (Bisazza et al. 1989; Plath et al. 2008c; Jeswiet et al. 2011b) and other taxa (Witte 2006b), and is a strong predictor of mating effort and mate choice in guppies (Dugatkin and Godin 1992; Jeswiet and Godin 2011). I carried out the trials for the two treatments in alternating order. A side bias was noted in a total of seven trials (four in Preference Test 1, three in Test 2), which were discarded. I tested 20 focal males (that showed no side bias) for each of the two treatments, and used different model males and stimulus females for each trial. None of the fish were re-used.

If male guppies copy the mating preferences of other males, then I should expect either a significant weakening or a reversal of a focal male's initial preference for a particular female following his observing a model male near the other, initially nonpreferred, female (Copying treatment) compared to his behaviour in the absence of a model male (Control treatment) (Dugatkin 1992; Dugatkin and Godin 1992).

\subsubsection{Controlling for shoaling behaviour}

Because guppies are shoaling fish (Houde 1997; Magurran 2005), I carried out a corollary control treatment testing for the possibility that any mate-copying behaviour 
observed in the aforementioned experiment could alternatively be owing to shoaling behaviour by focal males, expressed as more time spent on the side of the test aquarium where they had previously seen two fish (stimulus female and visible model male) than on the other side where they had seen one fish (stimulus female).

Following Dugatkin and Godin (1992), I tested for this possibility by using an identical protocol to the Copying treatment above, beginning at the viewing phase, except that the stimulus females in the end compartments were replaced with size-matched stimulus males (Table 5-2) to remove the sexual context. I quantified the time spent by the focal male in each of the two preference zones, whilst facing the stimulus male, as a measure of his shoaling preference. I tested 20 focal males, which were not re-used. The width $(10 \mathrm{~cm})$ of the preference zones in the test aquarium corresponds to about five body lengths, which is within the range of inter-individual distances observed in shoaling fish in nature (Pitcher and Parrish 1993).

I compared the time individual focal males spent associating with the stimulus male that was previously observed (during the viewing period) near another male in one of the two end compartments against the time spent associating with the other stimulus male, which was previously observed as being apparently alone. 
Table 5-2 Mean \pm SE (range) standard body length $(\mathrm{mm})$ of male guppies used in the shoaling control treatment of the mate-choice copying experiment. The result of an ANOVA test for homogeneity of body length among male types is shown.

\begin{tabular}{cccccc}
\hline Focal male & Model male & Hidden model male & Stimulus male 1 & Stimulus male 2 & ANOVA result \\
\hline $18.7 \pm 0.2$ & $18.9 \pm 0.2$ & $18.8 \pm 0.2$ & $19.4 \pm 0.2$ & $19.0 \pm 0.2$ & $F_{4,95}=1.76$, \\
$(17.2-21.0)$ & $(17.5-20.5)$ & $(17.1-20.4)$ & $(17.7-20.9)$ & $(17.2-20.4)$ & $P=0.14$ \\
\hline
\end{tabular}




\subsubsection{Statistical analysis}

All $t$-tests used were two-tailed and all statistical analyses were performed using the $\mathrm{R}$ statistical framework (R Core Team 2014).

To ascertain whether focal males modified their initial mating preference after having observed another male (the model) visually interact with the formerly nonpreferred female, I first calculated the proportion of time focal males spent associating with the initially-preferred and initially non-preferred females in Preference Test 1 and Preference Test 2 of each treatment, separately. I then used paired $t$-tests to compare the proportion of time that males spent with their initially non-preferred female in Preference Test 1 to that in Preference Test 2 for both treatments.

To compare between the two treatments, I calculated for each focal male a difference score for any change in mating preference between the matched preference tests $[$ Difference score $=($ Proportion of time near the initially non-preferred female in Preference Test 2 - Proportion of time near the initially non-preferred female in Preference Test 1)], following Jeswiet et al. (2011). A positive value denotes an increase in the proportion of time a focal male spent with the initially non-preferred female in the second preference test (taken as evidence for mate-choice copying, Dugatkin and Godin 1992). I compared the difference score between the two treatments using an independent $t$-test.

To test for the possibility that any observed copying effect could instead be explained by a shoaling response, I compared the time individual focal males spent associating with the stimulus male that was previously observed (during the viewing period) near another male in one of the two end compartments against the time spent 
associating with the other stimulus male, which was previously observed as being apparently alone using a paired $t$-test.

To complement the above analysis, I also compared the number of focal males that reversed their initial mating preference in Preference Test 2 in each treatment separately. As noted above, preference for a particular female was defined as a male spending more than $50 \%$ of his time associating with her. Following Dugatkin and Godin (1992), I compared frequencies of occurrence of mate-choice reversal using the $G$-test with Williams' correction (controlling for the expected false discovery rate (FDR) for multiple comparisons, Benjamini and Hochberg 1995).

Lastly, because any difference in overall sexual motivation by focal males between the paired preference tests may result in a change in preference between the tests, I compared any difference in the overall total time individual focal males spent near either stimulus female (as a proxy measure of their sexual motivation) between paired Preference Test 1 and Test 2 using a paired $t$-test for each treatment separately. Next, I calculated the relative change in the amount of time that the focal males spent near either of the stimulus females in Preference Test 1 compared to Test 2 as [Relative change $=$ (Total time spent near either female in Test 2 - Total time spent near either female in Test 1 /Total time spent near either female in Test 1) x 100] for each treatment separately. I compared the relative change in the time spent near either of the stimulus females between Test 1 and Test 2 between treatments using the Mann-Whitney U test. 


\subsubsection{Ethical standards}

The current study was approved by my institutional Animal Care Committee (protocols 10651 and B10-17), and adheres to the animal use and care guidelines of the Canadian Council on Animal Care and thus the laws of Canada.

\subsection{Results}

Overall, focal male guppies spent more time associating with the larger $($ mean $\pm \mathrm{SE}=$ $693.2 \pm 47.3 \mathrm{~s})$ than the smaller $(308.6 \pm 35.9 \mathrm{~s})$ of two stimulus females (paired $t$-test: $t_{39}$ $=8.15, P<0.001)$ in Preference Test 1 , thereby showing a strong baseline preference for large females. Males were consistent in their mating preference between the two consecutive preference tests when there was no copying opportunity available (paired $t$ test: $t_{19}=0.87, P=0.40$, Figure 5-2; see also Figure 5-3c). In contrast, focal males significantly increased their time spent near their initially non-preferred female, and therefore spent correspondingly less time near the other (initially preferred) female, after a copying opportunity was presented (paired $t$-test: $t_{19}=-5.14, P<0.001$, Figure $5-2 a$; see also Figure 5-3c). This behavioural response was reflected in a significant change in the mating preference of the focal males (i.e. difference score; $t$-test: $t_{38}=-4.91, P<$ 0.001 , Figure $5-2 b$ ), and thus in significantly more males reversing their initial mating preference between the paired preference tests ( $G$-test: $G_{1}=8.72, P=0.003$, Figure 5$2 c$ ), when a copying opportunity was present than when absent. Taken together, these results strongly indicate that focal male guppies 'copied' the apparent mate choice of a demonstrator (model) male. 

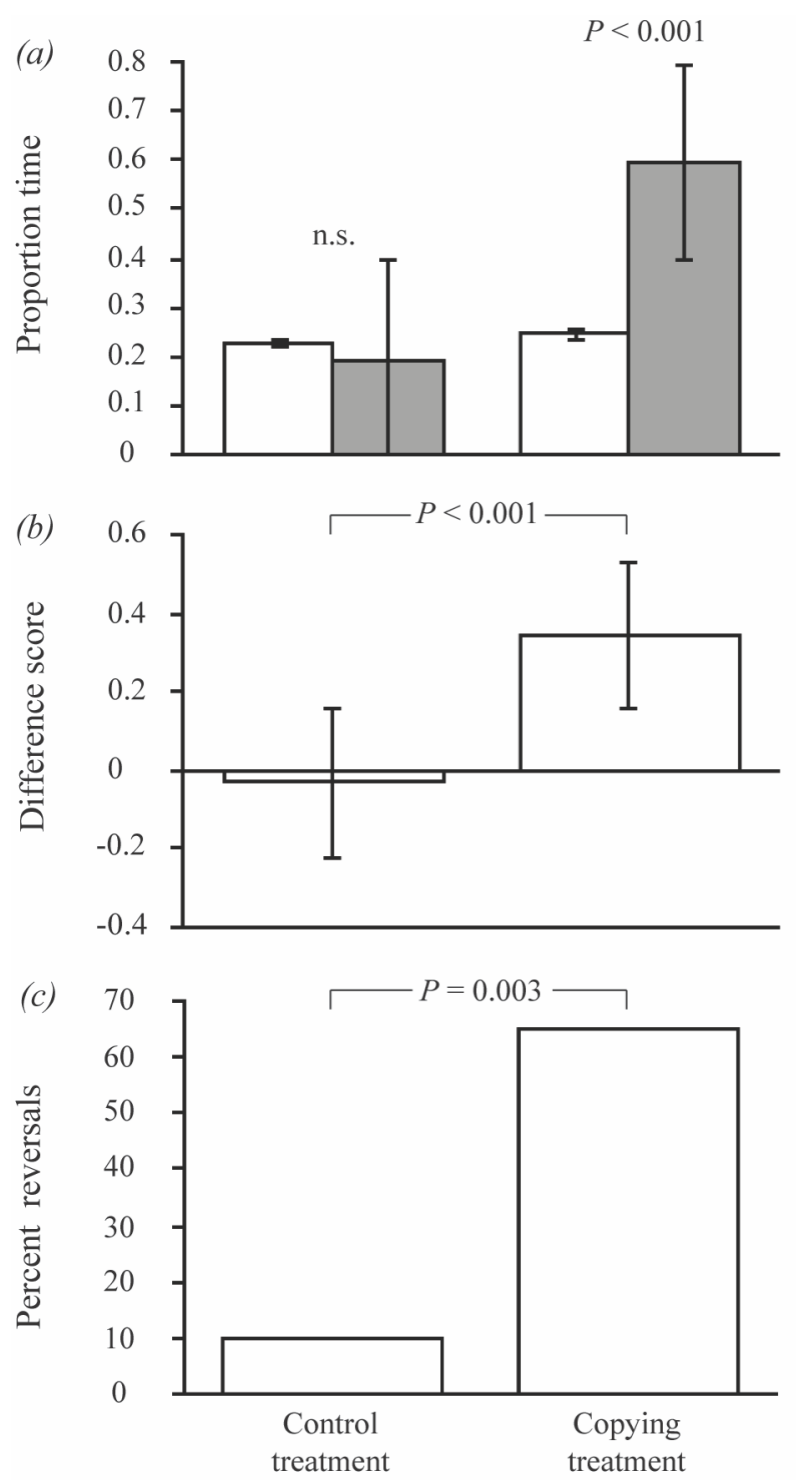

Figure 5-2 Mating preferences of focal males for the female that was initially not preferred in Preference Test 1 after they had observed that same female in either the absence (Control treatment; no copying opportunity) or the presence of a conspecific male model behind a clear Plexiglas partition (Copying treatment; copying opportunity) in Preference Test 2. Mating preferences are shown using three measures: $(a)$ mean $( \pm$ $\mathrm{SE})$ proportion of time that focal males spent associating with the initially non-preferred female in Preference Test 1 (open bar) and in Preference Test 2 (filled bar); $(b)$ mean ( \pm SE) preference difference score between Preference Test 2 and Preference Test 1 (a positive value denotes an increase in the focal male's preference for the initially nonpreferred female in Preference Test 2); and (c) percentage of males that reversed their initial preference (observed in Preference Test 1) in Preference Test 2. Significant $P$ values are shown for paired comparisons; n.s. denotes not significant $(P>0.05)$. 
Males were consistent in the amount of time they spent in the preference zone of either stimulus between the two consecutive preference tests when there was no copying opportunity available (paired $t$-test: $t_{19}=1.61, P=0.12$, Figure $5-3 a$ ). Focal males significantly decreased the time they spent near either stimulus female after a copying opportunity was presented (paired $t$-test: $t_{19}=3.21, P=0.004$, Figure 5-3a). I observed no significant difference in the change in the amount of time that males spent with either stimulus female from Preference Test 1 to Preference Test 2 between treatments (MannWhitney U test: $W=143, P=0.13$, Figure 5-3b), suggesting that the sexual motivation of focal males did not differ significantly between treatments. In the corollary treatment controlling for shoaling behaviour, focal male guppies spent significantly less time (312.6 $\pm 43.4 \mathrm{~s}$ ) associating with the stimulus male they had previously observed near another male (paired $t$-test: $\left.t_{19}=-2.16, P=0.04\right)$, and thus spent more time $(513.6 \pm 59.65 \mathrm{~s})$ with the other stimulus male they had not previously observed near another male. This result is contrary to that expected based on social attraction to an area where more fish had been previously observed. Therefore, my findings above, demonstrating mate-choice copying, cannot be explained by any shoaling behaviour exhibited by the focal males. 

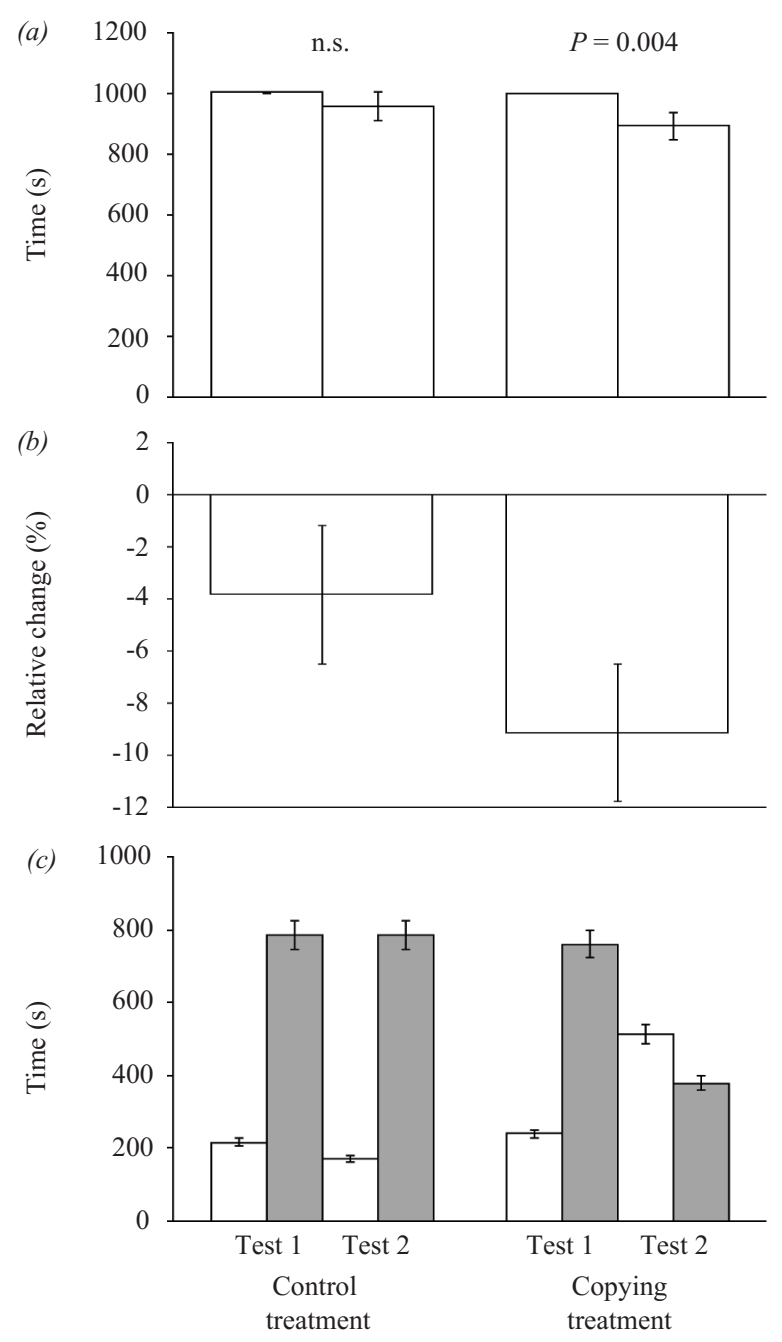

Figure 5-3 Comparison of the mating behaviour of focal males in Preference Test 1 compared to their mating behaviour in Preference Test 2 in either the absence of any copying opportunity or when a copying opportunity was present. Mating behaviour is shown as $(a)$ the mean \pm SE amount of time that the focal males spent associating with either of the stimulus females in Preference Test 1 and Preference Test 2, $(b)$ the mean \pm SE change in the amount of time that focal males spent associating with either stimulus female in Preference Test 2 relative to the amount of time they spent with either stimulus female in Preference Test 1 , and $(c)$ the mean \pm SE amount of time that focal males spent associating with the initially non-preferred female (open bars) and the initially preferred female (grey bars) in Preference Test 1 and Test 2 for each treatment separately. 


\subsection{Discussion}

In the current study, male guppies 'copied' the observed apparent mate choice of a nearby conspecific male (the demonstrator or model), as revealed by a significant shift in their initial preference for a particular female (typically the larger female) towards an increased preference for another female whom they had recently observed receiving sexual attention from a model male rival. In comparison, in the absence of any opportunity to copy, males were consistent over time (between the two consecutive preference tests) in their mating preferences, confirming similar findings in previous studies on the guppy (e.g. Jeswiet et al. 2011b; Godin and Auld 2013). Such mate-choice copying behaviour by male guppies thus cannot be explained by spontaneous temporal changes in their mating preference over time, any changes in sexual motivation, or by simple shoaling behaviour. Nor can it be explained by any differential sexual response or activity level of the stimulus females towards the focal or model males, because paired females in each trial were similarly gravid and thus unreceptive to male courtship (cf. Houde 1997) and both were exposed to a model male (one hidden and the other visible to the focal male in the copying opportunity treatment). My findings therefore strongly suggest that male guppies are sensitive to the presence and sexual behaviour of nearby rival males, and social information gathered resulted in males modifying their own mating preferences by copying the observed apparent mate choice of their rivals.

Although females from a number of Trinidadian guppy populations (e.g. Briggs et al. 1996; Dugatkin et al. 2002; Godin et al. 2005), including the Upper Aripo River (J.G.J. Godin, A. Miller and A.-C. Auge, unpubl. data), are known to mate-choice copy when given the opportunity to do so, my current study is the first to report evidence for 
male mate-choice copying in the Trinidadian guppy to my knowledge. A recent study (Makowicz et al. 2010b) with non-Trinidadian feral guppies did not find a reversal of male mating preference and concluded that mate-choice copying was not responsible for their results. Differences in the result of their study and mine can be attributed to differences in fish provenance, experimental design and measure of mating preference. In the Makowicz et al. (2010b) study, males were allowed to fully interact with both freeswimming stimulus females, which could lead to successful inseminations and thus an increase in actual sperm competition, whereas in my study focal males could only view rival males demonstrating sexual interest towards the initially non-preferred female and were unable to directly interact and copulate with either stimulus female. Additionally, Makowicz et al. measured preference as number of sexual acts directed towards each female, whereas I used time spent in each female's preference zone as a measure of preference (= association time), which is another predictor of actual mate choice in the guppy (Dugatkin and Godin 1992; Jeswiet and Godin 2011). In addition to my findings here, mate-choice copying by males has been previously demonstrated in only a few other species of fish (Schlupp and Ryan 1997; Witte and Ryan 2002; Frommen et al. 2009; Bierbach et al. 2011b) and in humans (Place et al. 2010; Yorzinski and Platt 2010), among species with traditional sex roles. Compared with females (reviewed by Witte 2006a; Vakirtzis 2011; Witte et al. 2015), this relative paucity of evidence for matechoice copying in males is likely owing to a female sex bias in studies of mate-choice copying to date, which perhaps reflects the traditional view that males are generally less choosy than females (Andersson 1994). Male mate-choice copying will undoubtedly be 
discovered in more species in the future, as it is now well established that males from diverse taxa also express mating preferences (Edward and Chapman 2011).

In the absence of nearby sexual rivals and copying opportunities, mating preferences in male Trinidadian guppies are consistent over time (current study; Jeswiet et al. 2011b; Godin and Auld 2013; Chapter 4) and among individuals are highly repeatable (Godin and Auld 2013; Chapter 4), possibly reflecting a heritable component (Boake 1989). Notwithstanding such consistency, their mating preferences can also be plastic and responsive to the ambient socio-sexual environment, as illustrated by the influence of the presence and sexual behaviour of nearby sexual rivals (and potential sexual competitors) on the mating preferences of focal males (current study; Chapters 67; Jeswiet et al. 2011b, 2012).

Copying the mate choice of another male is potentially associated with an increased risk of sexual and sperm competition, a cost which may however be partially offset by the reduced costs of mate sampling and assessment enjoyed by copiers in theory (Witte 2006a; Vakirtzis 2011; Witte et al. 2015). It has been suggested that one of the fitness-related benefits of male mate-choice copying is a reduced cost of finding sexually receptive females (Schlupp and Ryan 1997), which are relatively rare in natural populations of the Trinidadian guppy (Houde 1997). Although stimulus females in my current study were gravid and consequently sexually unreceptive, Trinidadian male guppies nonetheless sexually pursue, court and attempt to mate with previously-mated gravid females (Houde 1997; Guevara-Fiore et al. 2009; Jeswiet and Godin 2011; Godin and Auld 2013; Chapter 4) and can successfully inseminate unreceptive gravid females through forced sneak copulations (Pilastro and Bisazza 1999; Evans et al. 2003a) and sire 
offspring (Kelly et al. 1999; Herdman et al. 2004; Neff at al. 2008). Since male guppies primarily use olfactory cues to assess female reproductive status (Guevara-Fiore 2009), the model male's spatial proximity to and sexual interest in the nearby stimulus female in my behavioural trials likely signaled that female's (apparent) sexual receptivity or 'quality' as a potential mate to the focal male observer.

To my knowledge, this is the first study to demonstrate male mate-choice copying in the Trinidadian guppy. The modification of male mating preferences and the consequent alteration of social information conveyed in the presence of an audience of sexual rivals, as shown in the current study, has important implications for the evolution of social information use and the relative frequency of social copiers and non-copiers in a population (Dubois 2015). This work highlights the importance of the use of public information by males when choosing mates, and thus contributes to our relatively limited knowledge of male mate choice in general (Edward and Chapman 2011). Towards a more comprehensive understanding of the evolution of male mate choice, future empirical and theoretical studies should therefore place greater emphasis on the social environment and the use of public information by males in their mating decisions.

\subsection{Acknowledgments}

I thank the Director of Fisheries, Ministry of Agriculture, Land and Marine Resources of Trinidad and Tobago for permission to collect fish from the Upper Aripo River, G.E. Brown and C. Elvidge for assistance with fish collection, I.W. Ramnarine for logistical support in Trinidad, and C. Gabor, M. Plath and two anonymous reviewers for their constructive comments on the manuscript. 


\section{Chapter 6:}

\section{Do Male Trinidadian Guppies Adjust their Mate Choice in the Presence of a Rival Male Audience?}

This chapter is published as: Auld, H.L. and Godin, J.-G.J. 2015. Sexual voyeurs and copiers: social copying and the audience effect on male mate choice in the guppy. Behavioral Ecology and Sociobiology, 69: 1795-1807. It is reproduced here with permission from Springer-Verlag Berlin Heidelberg ${ }^{\circledR}$. 


\subsection{Abstract}

In most mating systems, males and females are commonly within signaling and receiving distance of conspecifics during courtship and mating activities. Although it is well known that females who observe sexual interactions between conspecifics will use public information obtained from these interactions when making their own mating decisions, much less is known about whether males use this type of information in making mating decisions. I used the Trinidadian guppy (Poecilia reticulata) to test whether males use public information to modify their mating preference for a given female in the presence of one or two sexual rivals (potential copiers). I show that males alter their mating preferences in response to the presence of an audience of sexual rivals, but find no evidence of a stronger audience effect when the number of sexual rivals increases. These results indicate that males pay attention to their immediate social environment in making mating decisions, and suggest that they avoid having another male copy their mate choice by weakening or even reversing their initial mating preference in the presence of eavesdropping male sexual competitors. My findings highlight the importance of social context and public information in male mate-choice decisions and have implications for the evolution of male mating preferences and of social information use in populations. 


\subsection{Introduction}

In most animal mating systems, sexual interactions between males and females commonly occur in the presence of nearby eavesdropping conspecifics (i.e. an 'audience', McGregor and Peake 2000). When sexually interacting with a particular female, a male (the 'demonstrator') may inadvertently reveal his mating preference to a nearby eavesdropping male rival, thereby conveying to the latter public information (sensu Danchin et al. 2004) about the sexual attractiveness and (or) receptivity of that female. If this public information is received by an audience male rival, it may subsequently be used by that male to copy the mate choice of the demonstrator male (Witte 2006a; Vakirtzis 2011; Witte et al. 2015), thereby increasing the risk of sperm competition and reduced share of paternity for the copied male (Wedell et al 2002; Witte 2006a). To date, male mate-choice copying has been demonstrated in only a few species (Schlupp and Ryan 1997; Witte and Ryan 2002; Frommen et al. 2009; Place et al. 2010; Yorzinski and Platt 2010; Bierbach et al. 2011b), including the guppy (Auld and Godin 2015; Chapter 5).

If males are at risk of being copied by sexual rivals and of incurring a resultant increase in their risk of sexual competition, then there should be selection on males to either minimize the inadvertent social transmission of cues about their choice of mates when in the presence of any audience male or to communicate false (deceptive) information about their mating preferences that directs the attention of audience males away from their preferred females (McGregor and Peake 2000; Dubois 2015). A change in the behaviour of a focal individual when in the presence of an audience is referred to as the 'audience effect' (McGregor and Peake 2000; Dablesteen 2005), which has been 
demonstrated in a sexual context in several species of fish (Dzieweczynski and Rowland 2004; Wong 2004; Dzieweczynski et al. 2005; Fisher and Rosenthal 2007; Plath and Schlupp 2008; Plath et al. 2008a, b, c, 2009, 2010; Ziege et al. 2008, 2009; Dzieweczynski and Walsh 2010; Makowicz et al. 2010a; Bierbach et al. 2013; Auld et al. 2015; Chapter 8) and in birds (Dubois and Belzile 2013). A sexual audience effect expressed by males may be a behavioural response to a perceived increase in their risk of sexual competition from sexual rivals (Kelly et al. 1999; Neff et al. 2008), as males usually exhibit a decreased preference for a preferred female in the presence of an audience of competitors (e.g. Plath and Schlupp 2008; Makowicz et al 2010a; Dubois and Belzile 2013; but see Bierbach et al. 2012 for an exception).

In general, it remains unknown whether the characteristics of an audience, such as the number of audience members or their phenotypes, influence the direction and(or) magnitude of the audience effect (see Dzieweczynski and Walsh (2010) and Bierbach et al. (2011a) for exceptions). If an audience comprises several individuals, and therefore many potential 'copiers', males may face an even greater risk of sexual competition than if there was only one audience member (cf. Wedell et al. 2002). A perceived increase in sexual competition risk may therefore result in a greater audience effect.

Here, using the Trinidadian guppy as a model study system, I tested the hypotheses that (i) males should modify their mate-choice behaviour in presence of an audience of sexual competitors (= audience effect), and (ii) the magnitude of their audience response should increase with an increase in the number of potential sexual competitors (audience males). 


\subsection{Methods}

\subsubsection{Study animals}

I used wild-caught Trinidadian guppies as a study species. Paired stimulus females used in the experiment described below were gravid and matched as closely as possible for their degree of abdominal distension (assessed by eye), an indicator of stage of pregnancy (Houde 1997); see Chapter 2 for rationale. However, they were chosen to differ in standard length (measured from tip of snout to caudal peduncle) by $20.8 \pm 1.0 \%$ (Table 6 1) to promote male discrimination between the females and to elicit a choice from the male (Dugatkin and Godin 1993; Jeswiet et al. 2011; Godin and Auld 2013; Chapter 4). All males used in any given behavioural trial were matched for standard length (measured with a metric scale) and colour ornamentation as closely as possible (assessed by eye, but later confirmed from analysis of their digital photograph; see Chapter 2 for methodology). 
Table 6-1 Mean \pm SE (range) standard body length $(\mathrm{mm})$ and body ornamentation score $(\%$ of male body covered by orange and black pigmentation) of guppies used in the three experimental treatments of the audience-effect experiment. Results of the ANOVA ( $F$-values), Kruskal-Wallis ( $\chi$-values), and $t$-tests for homogeneity of body length and colour score among male types, and results of $t$ tests comparing the body length of paired stimulus females between and within treatments, are shown. The Kruskal-Wallis test was used when residuals were not normally distributed.

\begin{tabular}{|c|c|c|c|c|c|c|c|c|}
\hline \multirow[b]{2}{*}{ Treatment } & \multicolumn{3}{|c|}{ Male length (mm) } & \multicolumn{3}{|c|}{ Male colour score $(\%)$} & \multicolumn{2}{|c|}{ Stimulus female length (mm) } \\
\hline & Focal & Audience 1 & Audience 2 & Focal & Audience 1 & Audience 2 & Large female & Small female \\
\hline No audience & $\begin{array}{c}18.5 \pm 0.3 \\
(16.7-21.7)\end{array}$ & - & - & $\begin{array}{c}6.5 \pm 0.5 \\
(2.9-12.5)\end{array}$ & - & - & $\begin{array}{c}25.7 \pm 0.5 \\
(22.5-31.5)\end{array}$ & $\begin{array}{c}21.8 \pm 0.4 \\
(17.0-27.7)\end{array}$ \\
\hline $\begin{array}{c}\text { Within } \\
\text { treatment } \\
\text { comparison }\end{array}$ & \multicolumn{3}{|c|}{-} & \multicolumn{3}{|c|}{-} & \multicolumn{2}{|c|}{$t_{54}=5.76, P<0.001$} \\
\hline $\begin{array}{c}1 \text {-male } \\
\text { audience }\end{array}$ & $\begin{array}{c}18.3 \pm 0.3 \\
(16.3-23.6)\end{array}$ & $\begin{array}{c}18.2 \pm 0.3 \\
(15.5-21.8)\end{array}$ & - & $\begin{array}{c}5.3 \pm 0.4 \\
(1.6-9.1)\end{array}$ & $\begin{array}{c}5.5 \pm 0.5 \\
(1.7-10.6)\end{array}$ & - & $\begin{array}{c}25.6 \pm 0.5 \\
(21.7-29.6)\end{array}$ & $\begin{array}{c}21.3 \pm 0.4 \\
(17.1-25.6)\end{array}$ \\
\hline $\begin{array}{c}\text { Within } \\
\text { treatment } \\
\text { comparisons }\end{array}$ & \multicolumn{3}{|c|}{$t_{56}=0.26, P=0.80$} & \multicolumn{3}{|c|}{$t_{56}=-0.34, P=0.73$} & \multicolumn{2}{|c|}{$t_{56}=7.42, P<0.001$} \\
\hline $\begin{array}{c}\text { 2-male } \\
\text { audience }\end{array}$ & $\begin{array}{c}18.0 \pm 0.2 \\
(15.9-20.5) \\
\end{array}$ & $\begin{array}{c}17.9 \pm 0.2 \\
(15.7-19.7) \\
\end{array}$ & $\begin{array}{c}18.2 \pm 0.3 \\
(15.8-20.9) \\
\end{array}$ & $\begin{array}{c}6.1 \pm 0.5 \\
(2.1-10.6) \\
\end{array}$ & $\begin{array}{c}5.9 \pm 0.7 \\
(0.5-14.3) \\
\end{array}$ & $\begin{array}{c}4.9 \pm 0.6 \\
(0.7-13.0) \\
\end{array}$ & $\begin{array}{c}25.7 \pm 0.6 \\
(22.4-32.5) \\
\end{array}$ & $\begin{array}{c}20.9 \pm 0.5 \\
(15.7-26.7) \\
\end{array}$ \\
\hline $\begin{array}{c}\text { Within } \\
\text { treatment } \\
\text { comparisons }\end{array}$ & \multicolumn{3}{|c|}{$F_{2,72}=0.42, P=0.66$} & \multicolumn{3}{|c|}{$F_{2,72}=1.55, P=0.22$} & \multicolumn{2}{|c|}{$t_{48}=6.4, P<0.001$} \\
\hline $\begin{array}{c}\text { Across } \\
\text { treatment } \\
\text { comparisons }\end{array}$ & $\begin{aligned} \chi_{2} & =1.78 \\
P & =0.41\end{aligned}$ & $\begin{array}{c}F_{2,76}=0.38 \\
P=0.69\end{array}$ & & $\begin{array}{c}F_{2,79}=2.34, \\
P=0.1\end{array}$ & $\begin{array}{c}F_{2,76}=0.07, \\
P=0.94\end{array}$ & & $\begin{aligned} \chi_{2} & =0.01 \\
P & =0.99\end{aligned}$ & $\begin{aligned} F_{2,79} & =0.845, \\
P & =0.04\end{aligned}$ \\
\hline
\end{tabular}




\subsubsection{General protocol}

See Chapter 2 for a description of the common general protocol.

\subsubsection{Experimental apparatus}

The dichotomous-choice experimental apparatus consisted of a clear Plexiglas test aquarium (40 x 20 × $25 \mathrm{~cm}$; L x W x H; Figure 6-1) flanked by two Plexiglas end containers (hereafter termed 'end compartments', 6 × $20 \times 25 \mathrm{~cm}$ ), which held the stimulus females. See Chapter 2 for more information on general test aquarium conditions. 
(a) Preference test 1

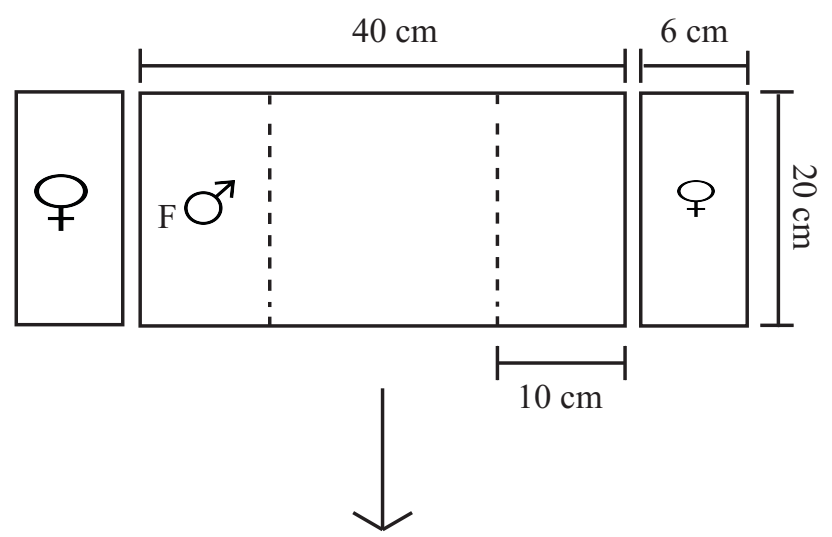

(b) Preference test 2

Treatment 1

No audience
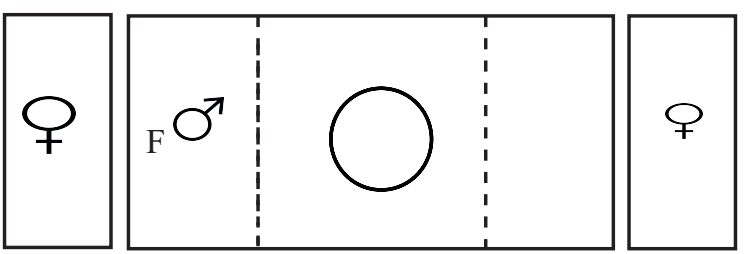

or

Treatment 2

One audience male
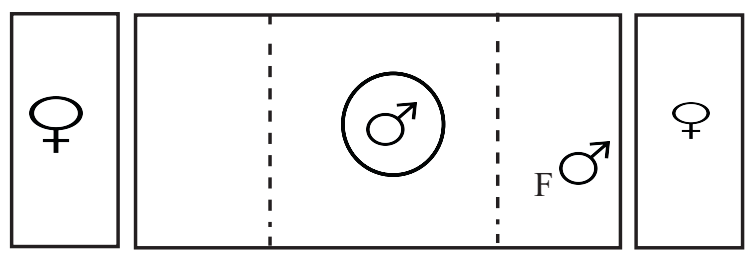

or

Treatment 3

Two audience males

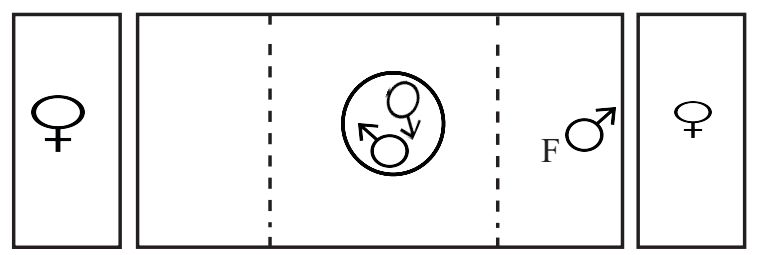

Figure 6-1 Schematic top view of the experimental apparatus. The central compartment held the focal male (F) and the 'audience' fish (constrained in a clear cylinder) and the end compartments held the stimulus females. In panel $(b)$, each circle represents a removable clear Plexiglas cylinder in Preference Test 2; one cylinder (most central - not shown) temporarily held the focal male and the other cylinder was either empty or held the 'audience' fish, depending on the treatment. The dashed lines in the test aquarium demarcate the $10-\mathrm{cm}$ preference zones 


\subsubsection{Experimental protocol}

The experimental protocol generally followed the one used by Plath et al. (2008a). The experiment comprised three treatments, which differed only in the membership size of the audience (either 0,1 or 2 rival male guppies). Briefly, for all treatments, a given trial consisted of two consecutive 20-min phases: $(i)$ an initial mating preference test (Preference Test 1) to establish the baseline preference of the focal male, and (ii) a second preference test (Preference Test 2) with either no audience (control treatment), an audience of one conspecific male or an audience of two conspecific males present (Figure 6-1). The control treatment (no audience) tested for consistency in male mating preference between the two consecutive preference tests in the absence of an audience, the one-member audience treatment determined whether a focal male's initial mate choice (in Preference Test 1) is altered by the presence of an audience of one potential sexual rival (in Preference Test 2), and the two-member audience treatment tested whether increasing the number (from 1 to 2 ) of potential sexual rivals in the audience would increase the magnitude of any observed audience effect on a focal male's mating preference.

All three treatments shared a common procedure as follows. A trial consisted of initially placing a focal male and the stimulus females in their respective compartments of the apparatus (Figure 6-1). The fish were left undisturbed for a 10-min acclimatization period, during which opaque screens prevented visual contact between the focal male and the stimulus females. Following acclimatization, I removed the opaque screens and allowed the focal male (constrained in the clear central cylinder) to observe the stimulus females in their respective end compartments for 5 min (viewing period). After this 
period, I released the focal male from the central cylinder and allowed him to 'choose' between the two stimulus females for $10 \mathrm{~min}$. Immediately following this choice period, I replaced the opaque screens and gently dip-netted the focal male to place him into the central cylinder. I then switched the positions of the two end compartments to control for any potential side bias in the male's preference behaviour. I removed the screens, released the male from the cylinder, and scored his mating preference for a second 10min period. Thus, the mating preference of the focal male was quantified over these two 10-min periods, for a total of 20 min (i.e. Preference Test 1), similar to the procedure used in Chapter 5.

Immediately following Preference Test 1, I replaced the opaque screens and returned the focal male to the central cylinder. For the control treatment (no audience), an empty clear Plexiglas cylinder ( $7 \mathrm{~cm}$ diam.) was gently placed adjacent to the focal male's cylinder in the centre of the test aquarium (Figure 6-1). For the one-member audience treatment, one male sexual rival was added to the cylinder to form an audience. In the two-member audience treatment, the audience comprised two male sexual rivals. Once the audience males were in place, I removed the opaque screens and allowed the focal male (whilst still in the cylinder) to view the stimulus females and, depending on the treatment, the audience male(s) for $10 \mathrm{~min}$. At the end of this viewing period, I released the focal male from his cylinder and recorded his mating preference for another 20-min (i.e. Preference Test 2). Audience male(s) remained in their cylinder throughout this mating preference test. Preference Test 2 was carried out exactly the same as Preference Test 1, except for the inclusion of the aforementioned particular treatments. 
I quantified the mating preference of individual focal males for either of the paired stimulus females as described in Chapters 2 and 5. For any preference test, the preferred stimulus female was considered the one with whom the focal male spent more than $50 \%$ of his total association time (summed over the paired females). This criterion, or slight variants of it, for mating preference is commonly used for poeciliid fishes (Bisazza et al. 1989; Plath et al. 2008c; Jeswiet et al. 2011b) and other taxa (Witte 2006b), and is a strong predictor of mating effort and mate choice in guppies (Dugatkin and Godin 1992; Jeswiet and Godin 2011). Trials for the treatments were carried out in alternating order. A side bias was noted in a total of 18 trials (15 in Preference Test 1, three in Test 2), which were discarded. I tested 28, 29 and 25 focal males (that showed no side bias) in Treatments 1, 2 and 3, respectively. I used different audience males and stimulus females for each trial. None of the fish were re-used.

\subsubsection{Control treatment for potential disturbance effect of an audience}

It is possible that any change in the behaviour of focal males in the presence of an audience could be owing to a general disturbance effect of any audience fish nearby rather than to perceived sexual competition (from rival male conspecifics) per se. To test for this possibility, I repeated the one-member audience treatment using a heterospecific adult zebrafish (Danio rerio), instead of a male guppy, as the audience. Adult zebrafish are similar in body size to adult Trinidadian guppies (Table 6-2), but do not resemble guppies morphologically or behaviourally nor overlap geographically with them. Therefore, male Trinidadian guppies are not likely to perceive adult zebrafish as sexual competitors or potential predators. Because of a limited availability of wild females at the 
Table 6-2 Mean \pm SE (range) standard body length ( $\mathrm{mm}$ ) of focal male guppies, audience zebrafish and paired stimulus female guppies used in the disturbance-effect control treatment. Results of paired $t$-tests comparing the body lengths of the focal male and audience zebrafish and the paired stimulus females, respectively, are shown.

\begin{tabular}{|c|c|c|c|}
\hline $\begin{array}{c}\text { Focal guppy } \\
\text { male }\end{array}$ & $\begin{array}{l}\text { Audience } \\
\text { zebrafish }\end{array}$ & $\begin{array}{l}\text { Large stimulus } \\
\text { female }\end{array}$ & $\begin{array}{l}\text { Small stimulus } \\
\text { female }\end{array}$ \\
\hline $\begin{array}{c}19.4 \pm 0.4 \\
(17.2-23.9)\end{array}$ & $\begin{array}{c}22.3 \pm 0.4 \\
(18.6-25.7)\end{array}$ & $\begin{array}{c}29.1 \pm 0.5 \\
(24.6-33.4)\end{array}$ & $\begin{array}{c}24.3 \pm 0.5 \\
(21.0-30.1)\end{array}$ \\
\hline \multicolumn{2}{|c|}{$t_{44}=-5.27, P<0.001$} & \multicolumn{2}{|c|}{$t_{44}=6.65, P<0.001$} \\
\hline
\end{tabular}


time of this control treatment, some (four) of the stimulus female guppies used were firstgeneration, lab-born offspring of wild-caught Upper Aripo River guppies; otherwise all other guppies used in the treatment were wild-caught. I tested 32 males, 9 of which exhibited side biases, which were discarded. Twenty-three focal males remained, which were not re-used.

\subsubsection{Statistical analysis}

All $t$-tests used were two-tailed and all statistical analyses were performed using the $\mathrm{R}$ statistical framework (R Core Team 2014). To ascertain whether focal males modified their initial mating preference after having observed an audience of rival male(s) nearby (audience effect), I first calculated the proportion of time focal males spent associating with the initially-preferred and initially non-preferred females in Preference Test 1 and Preference Test 2 of each treatment, separately. I then used paired $t$-tests to compare the proportion of time that males spent with spent with the initially-preferred female in Preference Test 1 to that in Preference Test 2 for each treatment separately.

To compare between treatments, I calculated for each focal male a difference score for any change in mating preference between the matched preference tests [Difference score $=($ Proportion of time near the initially-preferred female in Preference Test 2 - Proportion of time near the initially-preferred female in Preference Test 1)]. A negative value here denotes a decrease in the proportion of time a focal male spent with the initially-preferred female, and thus an increased relative preference for the other female, in the second preference test (taken as evidence for an audience effect if a male audience was present during Preference Test 2). I compared difference scores between 
treatments using a one-way ANOVA, followed by a Tukey's test for post-hoc multiple comparison of means.

To complement the above analysis, I also compared the number of focal males that reversed their initial mating preference in Preference Test 2 in each treatment separately. As noted above, preference for a particular female was defined as a male spending more than $50 \%$ of his time associating with her. Following Dugatkin and Godin (1992), I compared frequencies of occurrence of mate-choice reversal using the $G$-test with Williams' correction (controlling for the expected false discovery rate (FDR) for multiple comparisons, Benjamini and Hochberg 1995).

Lastly, because any difference in overall sexual motivation by focal males between the paired preference tests may result in a change in preference between the tests, I compared any difference in the overall total time individual focal males spent near either stimulus female (as a proxy measure of their sexual motivation) between paired Preference Test 1 and Test 2 using a paired $t$-test for each treatment separately. Next, I calculated the relative change in the amount of time that the focal males spent near either of the stimulus females in Preference Test 1 compared to Test 2 as [Relative change $=$ (Total time spent near either female in Test 2 - Total time spent near either female in Test 1 /Total time spent near either female in Test 1) x 100] for each treatment separately. I compared the relative change in the time spent near either of the stimulus females between Test 1 and Test 2 between treatments using a one-way ANOVA, followed by the Tukey test for multiple comparisons. 


\subsubsection{Ethical standards}

The current study was approved by my institutional Animal Care Committee (protocols 10651 and B10-17), and adheres to the animal use and care guidelines of the Canadian Council on Animal Care and thus the laws of Canada. 


\subsection{Results}

Overall, focal male guppies spent more time associating with the larger $(558.0 \pm 22.8 \mathrm{~s})$ than the smaller $(340.4 \pm 19.7 \mathrm{~s})$ of the two stimulus females (paired $t$-test: $t_{104}=5.49, P$ $<0.001)$ in Preference Test 1 , thereby demonstrating a strong baseline preference for large females. Males were consistent in their mating preference between the paired preference tests in the absence of an audience (paired $t$-test: $t_{27}=0.66, P=0.51$, Figure 6$2 a$; see also Figure 6-3a). In comparison, males significantly decreased the time they spent near their initially-preferred female when one audience male (paired $t$-test: $t_{28}=$ 5.86, $P<0.001$, Figure 6-2a; see also Figure 6-3a) or two audience males (paired $t$-test: $t_{24}=5.44, P<0.001$, Figure 6-2a; see also Figure 6-3a) was present. This male response to a conspecific male audience was reflected in a significant change in their preference difference score in the expected direction when an audience was present (one-way ANOVA: $F_{2,79}=8.13, P<0.001$, Figure 6-2b). Although the preference difference scores of focal males in the presence of a 1-male audience $(P=0.001)$ and 2-male audience $(P=$ 0.006 , Figure 6-2b) differed significantly from those in the control treatment (no audience) as expected, they did not differ from each other $(P=0.91)$. Reinforcing the above evidence for an audience effect, the number of males reversing their initial mating preference between the paired preference tests differed between treatments ( $G$-test: $G_{a d j 2}$

$=6.65, P=0.04$, Figure 6-2c). Proportionally more males reversed their mating preference in the presence of a 1-male audience $\left(G_{a d j 1}=5.04, P=0.03\right)$ and a 2 -male audience $\left(G_{a d j 1}=5.42, P=0.02\right)$ than when no audience was present (control), as expected (Figure 6-2c). However, contrary to expectation, there was no difference in mate-choice 
reversal frequencies, and thus the magnitude of the observed audience effect, between when one or two audience males were present $\left(G_{a d j 1}=0.007, P=0.47\right.$, Figure 6-2c).

In the zebrafish control treatment for a general disturbance effect, the proportion of time focal males spent near their initially-preferred female (in Preference Test 1) did not differ significantly from that in the paired Preference Test 2 whilst in the presence of an heterospecific (zebrafish) audience (paired $t$-test: $t_{22}=1.98, P=0.06$; Figure 6-2a). Therefore, the zebrafish audience did not significantly affect the mating preference of focal male guppies. Consequently, focal male mating difference scores and the proportion of male guppies reversing their initial mating preference between the paired preference tests did not differ between the zebrafish audience treatment and the control (no audience) treatment (Tukey HSD: $P=0.94$, Figure $6-2 b ; G_{a d j 1}=0.16, P=0.69$, respectively, Figure 6-2c). However, the responses of focal males [both their difference scores (Figure 6-2b) and proportion of males reversing their initial mating preference (Figure 6-2c)] to the presence of either one (Tukey HSD: $P=0.01$, Figure 6-2b; $G_{a d j 1}=$ $6.69, P=0.01$, Figure 6-2c, respectively) or two (Tukey HSD: $P=0.047$, Figure 6-2b; $G_{a d j 1}=6.91, P=0.009$, respectively, Figure 6-2c) audience male guppies were significantly greater than their responses to the presence of a zebrafish audience. These results suggest that the strength of the above-observed effect of a conspecific male audience on male guppy mating preferences is specific to the nearby presence of conspecific sexual rivals, and cannot be explained by any general disturbance effect of the presence of any fish. 


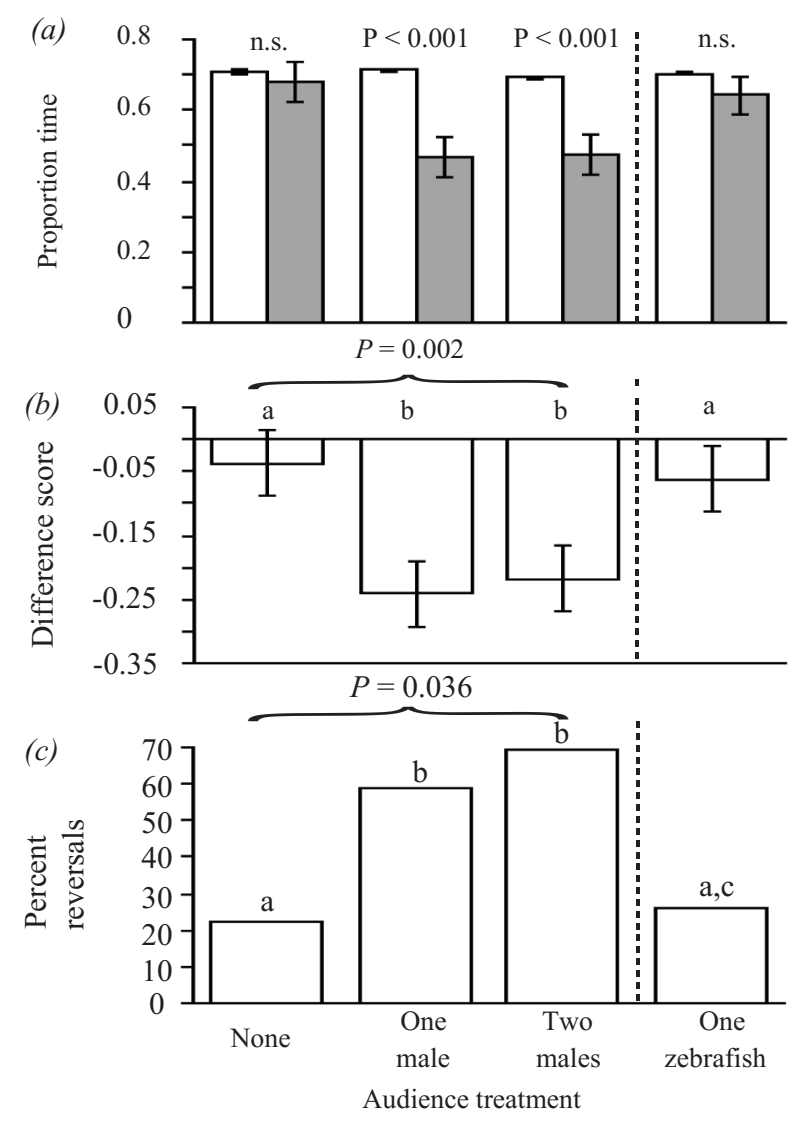

Figure 6-2 Comparison of the mating preferences of focal males for the particular female initially preferred in Preference Test 1 compared to their preferences for the same female in the paired Preference Test 2 when not being observed by an audience conspecific male (no audience) or being observed by either an audience one conspecific male, two conspecific males, or one heterospecific zebrafish (control for a general disturbance effect). The vertical dashed line separates the results obtained from the three treatments of Experiment 2 from the control (zebrafish audience) treatment. The mating preferences of focal males are shown using three measures: $(a)$ mean $( \pm \mathrm{SE})$ proportion of time that focal males spent associating with the initially-preferred female in Preference Test 1 (open bar) and in Preference Test 2 (filled bar); (b) mean ( \pm SE) preference difference score between Preference Test 2 and Preference Test 1 (a negative value denotes a decrease in the focal male's preference for the initially-preferred female in Preference Test 2); and (c) the percentage of males that reversed their initial preference (observed in Preference Test 1$)$ in Preference Test 2 . Only significant comparisons $(P<0.05)$ are shown in panel (a). In the other two panels, histogram bars with dissimilar letters are significantly different $(P<0.05)$ from each other. The multiple paired comparisons in panels $\mathrm{b}$ and c were carried out using paired $t$-tests and the $G$-test, respectively, with the FDR for multiple comparisons controlled for. 
Focal males spent less time with either stimulus female in Preference Test 2 compared to Preference Test 1 for all treatments (paired $t$-tests: all $P<0.02$, Figure 6-3a; see also Figure 6-3c). Overall, the relative change in the total amount of time that males spent with either stimulus female differed across treatments (one-way ANOVA: $F_{3,101}=$ $12.9, P<0.001$, Figure $6-3 b$ ), potentially suggestive of a change in sexual motivation over time that differed in magnitude between treatments. A post-hoc Tukey's HSD test revealed that the decrease in total association time near stimulus females exhibited by focal males when no audience present (control) was significantly less than that for when one $(P<0.001)$ or two $(P<0.001)$ audience male guppies are present and when a heterospecific zebrafish audience is present $(P<0.001)$. Because the observed decrease in the total time focal males spent with either stimulus female between paired preference tests did not differ between the heterospecific zebrafish audience treatment and either the one audience male guppy treatment $(P=0.73)$ or the two audience male guppy treatment $(P=0.98)$, and because the zebrafish audience treatment did not show an audience effect (Figure 6-2a), the aforementioned evidence for a guppy audience effect (Figure 6-2; see also Figure 6-3) cannot be explained simply by any change in male sexual motivation among the audience treatments. 

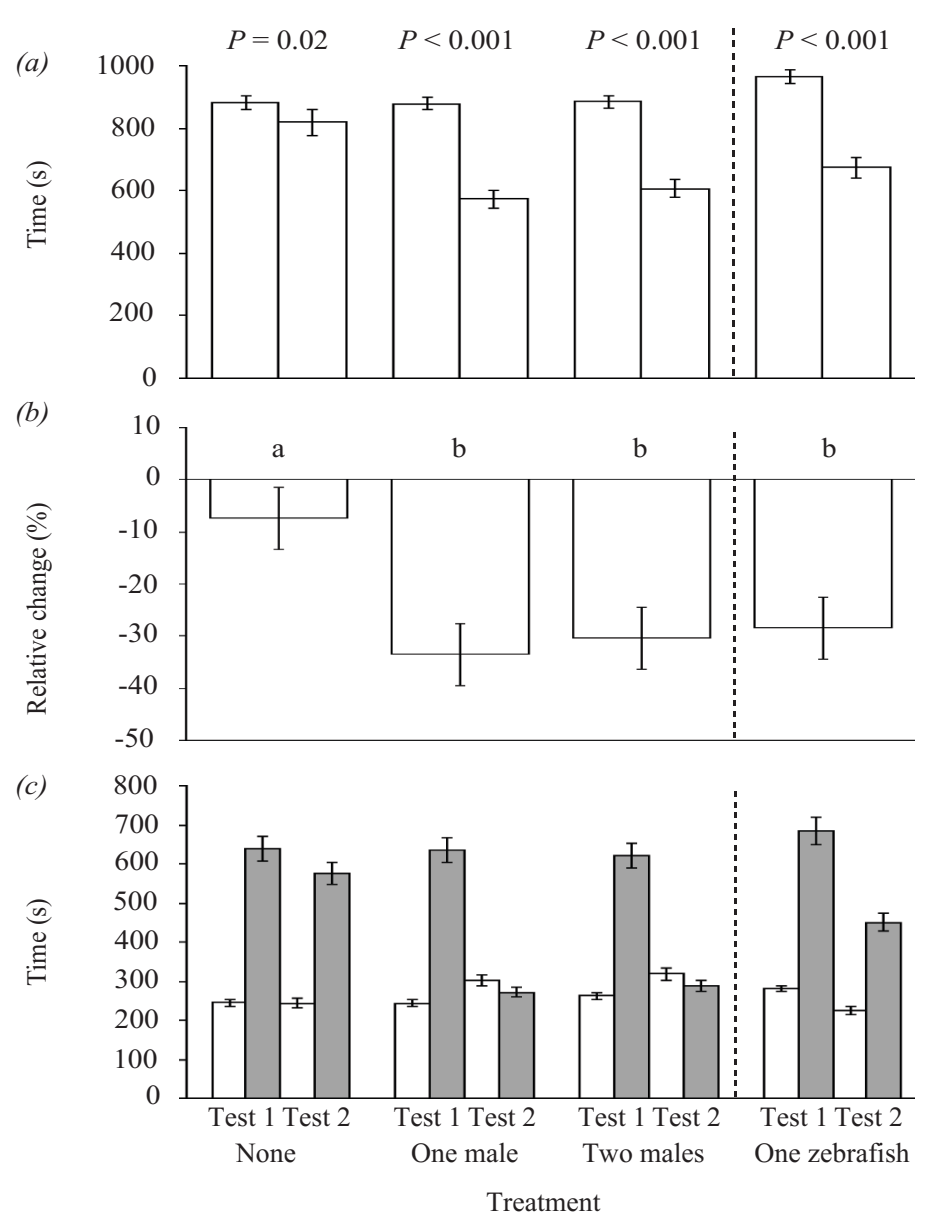

Figure 6-3 Comparison of the mating behaviour of focal males in Preference Test 1 compared to their mating behaviour in Preference Test 2 when either not being observed by an audience conspecific male (no male; Treatment 1 ) or being observed by either an audience one conspecific male (one male; Treatment 2), two conspecific males (two males; Treatment 3), or one heterospecific zebrafish (control for a general disturbance effect). The vertical dashed line separates the results obtained from the three guppy male audience treatments from the control (zebrafish audience) treatment. The mating behaviour of focal males is shown as $(a)$ the mean $\pm \mathrm{SE}$ amount of time that the focal males spent associating with either of the stimulus females in Preference Test 1 and Preference Test 2, $(b)$ the mean \pm SE change in the amount of time that focal males spent associating with either stimulus female in Preference Test 2 relative to the amount of time he spent with either stimulus female in Preference Test 1, and (c) the mean $\pm \mathrm{SE}$ amount of time that focal males spent associating with the initially non-preferred female (open bars) and the initially preferred female (grey bars) in Preference Test 1 and 2 for each treatment separately. Histogram bars with dissimilar letters are significantly different $(P$ $<0.05)$ from each other. The multiple paired comparisons in panel $(a)$ were carried out using the Tukey Test. 


\subsection{Discussion}

In the absence of nearby sexual rivals and copying opportunities, mating preferences in male Trinidadian guppies are consistent over time (current study; Chapter 4; Jeswiet et al. 2011b; Godin and Auld 2013). Notwithstanding such consistency, their mating preferences can also be plastic and responsive to the ambient socio-sexual environment, as illustrated by the influence of the presence and sexual behaviour of nearby sexual rivals (and potential sexual competitors) on the mating preferences of focal males (Chapter 5; Jeswiet et al. 2011b, 2012).

Copying the mate choice of another male (Chapter 5) is potentially associated with an increased risk of sexual and sperm competition, a cost which may however be partially offset by the reduced costs of mate sampling and assessment enjoyed by copiers in theory (Witte 2006a; Vakirtzis 2011; Witte et al. 2015). However, for males being copied, the cost of sexual competition is potentially significant because the level of multiple mating and mixed paternity in wild females is very high in natural Trinidadian guppy populations (Kelly et al. 1999; Neff et al. 2008) and some degree of last-male sperm precedence (Evans and Magurran 2001; Neff and Wahl 2004) would increase sexual competition costs to the male being copied in relation to the copier. Because of this potential high fitness cost of being copied, selection should thus favour males that are able to accurately assess their ambient risk of sexual competition and modify their behaviour to reduce the risk of being copied (Wedell et al. 2002; Dubois 2015).

As expected if being copied by a rival male is associated with a cost of sperm competition, focal males that were observed by either one or two conspecific audience males significantly decreased and reversed their initial preference for a particular female, 
thus shifting their relative preference to another female that they did not prefer initially, compared with focal males in the absence of an audience (whose mating preferences remained consistent over time). These results cannot be explained by any changes in the sexual motivation of focal males. Interestingly, the magnitude of the audience effect was not affected by the number of audience members ( 1 vs. 2 conspecific male rivals), as might be expected if males were sensitive to a perceived increase in sperm competition risk. It is unlikely that cognitive constraints on detecting a small difference in the number of audience males ( 1 vs. 2) can explain the observed lack of response to an increase in audience membership size, because guppies possess an innate ability to discriminate between small numbers (Piffer et al. 2013). More likely, the increase in audience membership size from one to two rival males represents too small of an increase in sperm competition risk or intensity to influence the focal male's mating behaviour (cf. Jeswiet et al. 2012). It is also possible that sperm competition is not a factor driving the audience effect.

The observed audience effect on male guppy mating preferences appears to be specific to an audience of conspecific rival males (that is, potential sexual competitors) and cannot be explained as a simple response to any type of audience fish (i.e. not a disturbance effect), because an audience of unfamiliar heterospecific zebrafish did not elicit a significant change in focal male guppy mating preferences. Alternatively, it could be argued that a perceived interest in one female over another by a conspecific male could lead to either copying behaviour, as shown in Chapter 5, or avoidance behaviour (Bierbach et al. 2011b; Jeswiet et al. 2011b). However, this explanation is unlikely because the audience male(s) was(were) constrained within a Plexiglas cylinder 
equidistant between the paired stimulus females and not sexually interacting with them, thus minimizing the chance that the focal male's behaviour was influenced by any perceived mate preference in the audience male(s).

One functional hypothesis (Plath and Schlupp 2008) proposed to explain an audience effect on male mate choice is that it can be attributed to males avoiding aggressive encounters with each other; this hypothesis assumes shared mating preferences between the audience and focal males. However, wild male guppies differ consistently in their individual mating preferences (Godin and Auld 2013; Chapter 4) and a recent comparative study across poeciliid fish species (Bierbach et al. 2013) did not reveal a correlation between aggressiveness and audience effects, suggesting that aggression avoidance is not the cause of observed audience effects. An alternative hypothesis to the one above proposes that males reduce their risk of being copied, and thereby reduce their risk of sexual competition, by shifting their sexual attention away from a preferred female in the presence of a rival male audience (Plath et al. 2008c). Overall, my findings for male mate-choice copying (Chapter 5) and an audience effect (current study) are consistent with this latter hypothesis. Dubois and Belzile (2012) also proposed that monogamous males might shift their preference away from a preferred female in the presence of a sexual competitor to reduce the risk of being rejected by the female; however, this hypothesis does not apply to male guppies as they are not monogamous.

The modification of male mating preferences and the consequent alteration of social information conveyed in the presence of an audience of sexual rivals, as shown in the current study, has important implications for the evolution of social information use 
and the relative frequency of social copiers and non-copiers in a population (Dubois 2015). My work highlights the importance of the use of public information by males when choosing mates, and thus contributes to our relatively limited knowledge of male mate choice in general (Edward and Chapman 2011). Towards a more comprehensive understanding of the evolution of male mate choice, future empirical and theoretical studies should therefore place greater emphasis on the social environment and the use of public information by males in their mating decisions.

\subsection{Acknowledgments}

I thank the Director of Fisheries of Trinidad and Tobago for permission to collect fish from the Upper Aripo River, G.E. Brown, C. Elvidge, B. Daniel, R. Mahibir and K. Deonarnsingh for assistance with fish collection, and A. Miller and C. Elvidge for assistance with data collection. 


\section{Chapter 7:}

\section{Male Mate Choice is Influenced by the Phenotype of Sexual \\ Rivals}

This chapter has been provisionally accepted, conditional upon satisfactory completion of minor revisions, in the journal Behavioral Ecology. 


\subsection{Abstract}

In populations with male mate-choice copying, males may mitigate their risk of sexual competition by reducing their preference for a particular female in the presence of sexual rivals (audience). Because of the cost of missed mating opportunities from such an audience effect, males should reduce their mating preference to a greater extent in the presence of more sexually competitive rival compared with less competitive ones. I tested this hypothesis using the Trinidadian guppy (Poecilia reticulata). I compared a focal male's baseline mating preference for either of two stimulus females, which differed in overall body size, in the absence of any sexual rival to his preference for the same females in the presence of a sexual rival using dichotomous-choice tests. Focal and audience males differed in body length and proportion of their body covered in orange and black pigmentation, two sexually-selected traits in the guppy. In the presence of a larger rival, focal males exhibited a greater reduction in preference for their initially preferred female compared to focal males in the presence of a smaller rival, irrespective of whether the latter male was more or less colour ornamented than the focal. The strength of the initial mating preference of focal males and the magnitude of the audience effect were significantly positively correlated when the audience male was larger than the focal male. Male guppies are thus sensitive to the phenotype of nearby males and alter their preference for a particular mate to a greater extent in the presence of relatively larger, and more sexually attractive and competitive, males compared to smaller ones. 


\subsection{Introduction}

The social environment can shape the behaviour of animals, with important implications for their fitness, sexual selection and social evolution (e.g. Wolf et al. 1999; McGlothlin et al. 2010; Oh and Badyaev 2010; Mautz and Jennions 2011; Callander et al. 2013; Gasparini et al. 2013). In such environments, individuals may supplement their own personal information about local ecological conditions with public information (sensu Danchin et al. 2004) by eavesdropping on social interactions between other animals and their behaviour (Valone 2007). In doing so, bystanding eavesdroppers are thus able to acquire relatively inexpensive public information, which they may later use in making their own behavioural decisions (Valone 2007). For example, with public information acquired through eavesdropping, an individual may decide to copy the behaviour of other individuals or to behave independently (Valone 2007; Nordell and Valone 1998; Danchin et al. 2004; Witte et al. 2015; Chapter 5). While copying the behaviour of another individual (i.e. the demonstrator) can provide benefits to the copier (e.g. reduced sampling costs), there can also be associated costs with copying for both the demonstrator and copier, such as increased resource competition and risk of sperm competition (Nöbel and Witte 2013; Dubois 2015; Witte et al. 2015; Castellano et al. 2016). When demonstrators and eavesdroppers compete for the same limited resources (e.g. mates, food), demonstrators could benefit from withholding or reducing social information, generated either inadvertently or intentionally through their actions, so as to limit or prevent the use of social information by eavesdroppers who could otherwise use such information to copy the demonstrators' behavioural decisions (Dubois 2015). Therefore, in the presence of eavesdropping competitors, demonstrator individuals should be 
expected to adjust their behaviour in a manner that minimizes potential costs associated with being copied (Nordell and Valone 1998; Dubois 2015; Castellano et al. 2016). A change in the behaviour of a focal animal in response to the presence of non-interacting bystanders is referred to as an 'audience effect' (Dablesteen 2005).

Audience effects have been documented for a variety of contexts, including foraging, predator avoidance and mate choice (e.g. Evans and Marler 1994; Pollick et al. 2005; Le Roux et al. 2008; Dubois and Belzile 2013; Dzieweczynski et al. 2005; Chapter 6). Audience effects are relatively common in polygamous or promiscuous mating systems species wherein individuals have opportunities to observe the mating behaviour of others and to copy their mate choice under certain conditions (e.g. Dugatkin 1992; Dugatkin et al. 1992; Schlupp and Ryan 1997; Schlupp et al. 1997; Witte et al. 2002, 2006a; Auld and Godin 2015; Chapter 5). An increasing body of work, mainly on polygamous fishes, has demonstrated that the mere presence of an audience of one or more sexual competitors can influence a male's mating preference and mating tactics (e.g. Dzieweczynski et al. 2005; Plath et al. 2008a,b, 2009; Ziege et al. 2008, 2009; Dzieweczynski and Walsh 2010; Mautz and Jennions 2011; Auld et al. 2015; Auld and Godin 2015; Chapters 6 and 8) and sperm allocation strategy (Nöbel and Witte 2013).

Despite growing interest in the effects of a rival male audience on male mating preferences, relatively little is known about the potential influence of differences in phenotype between eavesdropping bystanders (the audience) and demonstrators or actors (individuals at risk of being copied) on the magnitude of such audience effects (but see Dzieweczynski and Walsh 2010, Bierbach et al. 2011a, 2015). Of particular interest in this context is how the perceived sexual attractiveness and(or) sexual competitive ability 
of a rival audience male influences the extent to which a focal male (the demonstrator) alters his mating preference. For example, male Atlantic mollies (Poecilia mexicana) reduce their initial mating preference for a particular female to a greater extent when in the presence of a familiar, sexually-active and larger conspecific male (Bierbach et al. 2011b), and bolder males exhibit a greater audience effect than shyer ones (Bierbach et al. 2015). However, altering one's mating preference for a particular female in response to an audience can potentially be costly if it results in an increased likelihood of being subsequently rejected as a mate by that same female (Castellano et al. 2016). Therefore, if males flexibly adjust their mating behaviour in response to a perceived risk of pre- or post-copulatory sexual competition, then they should do so in a cautious, graded manner that reflects an optimal trade-off between the cost of being copied by an audience (i.e. increased risk of sperm competition) and the cost of being subsequently rejected as a mate by one's initially-preferred female (Dubois 2015; Castellano et al. 2016). I hypothesize that selection should thus favour males who are sensitive to the phenotypes of nearby audience male rivals (the audience) and who strategically modify their mating preference for an initially-preferred female depending on the relative sexual attractiveness and(or) sexual competitiveness of any nearby sexual male rival. Here, I used the Trinidadian guppy (Poecilia reticulata) as a model species to experimentally test this hypothesis.

In the presence of an audience of either one or two potential sexual competitors, male guppies reduce their preference for an initially-preferred female (Makowicz et al. 2010b; Bierbach et al. 2013; Auld and Godin 2015; Chapter 6), presumably to minimize the risk of another male copying their mate choice (Auld and Godin 2015; Chapter 5) 
which in turn would potentially elevate the risk of sperm competition and decrease their share of paternity (Wedell et al. 2002; Witte 2006a). I previously demonstrated that this audience effect on male mating preferences in the guppy is not simply the result of any temporal spontaneous changes in male mating preference, but rather is specific to an audience of conspecific male and not to a general disturbance effect of any fish being present near the focal male (Auld and Godin 2015; Chapter 6). Male guppies vary in the extent to which they alter their mating preference in the presence of a sexual rival (e.g. Makowicz et al. 2010b; Auld and Godin 2015; Chapter 6). Sexual rivals also vary both in their ability to attract and mate with females and the ability of their sperm to fertilize eggs (Brooks and Endler 2001; Brooks 2002; Pilastro et al. 2002; Locatello et al. 2006; Auld et al. 2016; Chapter 3).

In my study population in Trinidad, large and(or) more colour ornamented males are expected to be more sexually attractive and competitive than smaller and(or) drabber males because females prefer larger and more ornamented males as mates (Magellan et al. 2005; Auld et al. 2016; Chapter 3; but see Schwartz and Hendry 2007) and more ornamented males (possessing more orange and black colour on their body) produce faster and more viable sperm than drabber males (Locatello et al. 2006), suggesting that more ornamented males have a competitive advantage in sperm competition over less ornamented ones. Irrespective of body ornamentation, large male guppies might also outcompete smaller rival males for access to females through sneak copulation, because burst swimming and body rotational velocities are positively correlated with body size in this species (Ghalambor et al. 2004). 
In the current study, I therefore used the differences in these two sexually-selected traits (body length and body ornamentation) between focal and audience males as proxy measures of their relative sexual attractiveness to females and their sexual competitiveness. I used the sequential dichotomous-choice test paradigm (Chapter 2) to test for an effect of an audience (one rival conspecific male) on the mating preference of focal male guppies for either a large or a small stimulus female. Dichotomous-choice tests are commonly used to assess mate choice in animals (e.g. Witte 1996b; Lehtonen and Lindström 1998; Walling et al. 2010; Jeswiet et al. 2011a,b; Jeswiet and Godin 2011). For each mate choice trial, I experimentally varied the perceived sexual attractiveness and(or) sexual competitiveness of the audience male, as measured by his body length and body ornamentation (amount of orange and black colour on his body), relative to that of the focal male to ascertain whether the magnitude of any observed audience effect is dependent on the relative difference in the sexual phenotype of focal and audience males. I predicted that (i) focal males should on average exhibit an initial preference for the larger (i.e. potentially more fecund, Reznick and Endler 1982; Kelly et al. 1999; Herdman et al. 2004; Chapters 5 and 6) of two available stimulus females, (ii) focal males should reduce their initial preference for a particular female in the presence of an audience rival male compared with its absence (i.e. exhibit an audience effect), and (iii) the magnitude of any observed audience effect should increase as a function of both the differences in the body length and(or) ornamentation between the audience and focal males and the strength of the focal male's initial preference for a particular stimulus female, which I assume is indicative of his assessment of that female's reproductive value and the potential cost of lost paternity if his mate choice is copied by an eavesdropping 
rival male (Dosen and Montgomerie 2004b; Jeswiet et al. 2012; Godin and Auld 2013; Chapter 5).

\subsection{Methods}

\subsubsection{Study animals}

I used laboratory-reared Trinidadian guppies as a study species. See Chapter 2 for a rationale.

\subsubsection{General protocol}

See Chapter 2 for a description of the common general protocol.

\subsubsection{Experimental apparatus}

My dichotomous-choice experimental apparatus consisted of a clear Plexiglas test aquarium ( $40 \times 20 \times 25 \mathrm{~cm}$; L x W x H) flanked by two Plexiglas end containers (hereafter termed 'end compartments', 6 × $20 \times 25 \mathrm{~cm}$ ), which held the stimulus females (Figure 7-1). See Chapter 2 for more information on general test aquaria conditions.

\subsubsection{Experimental protocol}

The experiment comprised four treatments, which differed only in the body length and(or) body ornamentation of the audience male relative to the body length and ornamentation of the focal male (Table 7-1). I manipulated the sexual attractiveness and(or) competitiveness of the audience male in any given mate choice trial by preselecting the audience male to be either smaller and less ornamented than the focal male 
(Treatment 1), smaller but more ornamented than the focal male (Treatment 2), larger but less ornamented than the focal male (Treatment 3), or larger and more ornamented than the focal male (Treatment 4). Males were initially measured for their standard body length using a metric scale and their body ornamentation assessed as closely as possible (by eye, but later confirmed from analysis of their digital photograph; see Chapter 2) and categorized into one of the four treatments (Table 7-1). The above treatment combinations of body length and ornamentation for the audience males reflect their occurrence in nature, as these two phenotypic traits in wild male guppies in my study population are highly variable, normally distributed and covary only weakly $(r=0.152, N$ =672; Auge et al. 2016). The four experimental treatments are thus designed to test for an audience effect on the mating preference of focal males and whether the magnitude of any observed audience effect is influenced by the relative differences in sexual attractiveness and(or) competitiveness of the focal and audience males, as measured by differences in their body length and body ornamentation (cf. Introduction). 
(a) Preference test 1

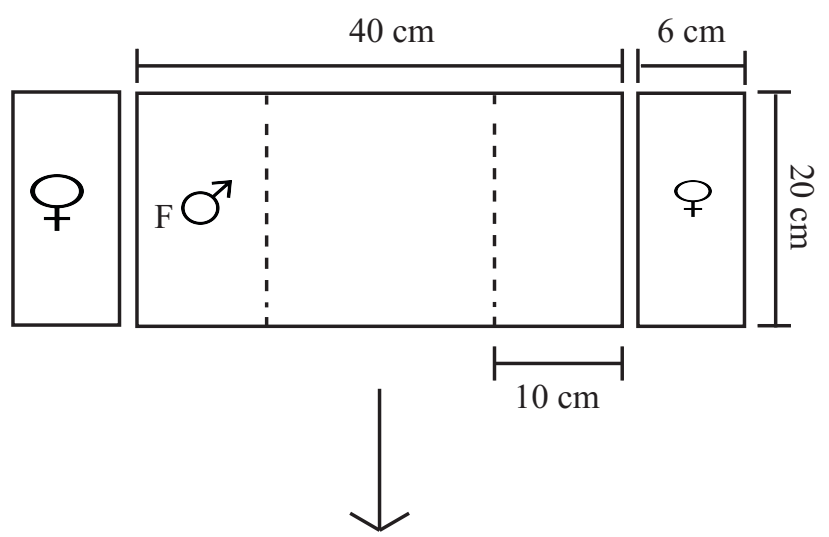

(b) Preference test 2

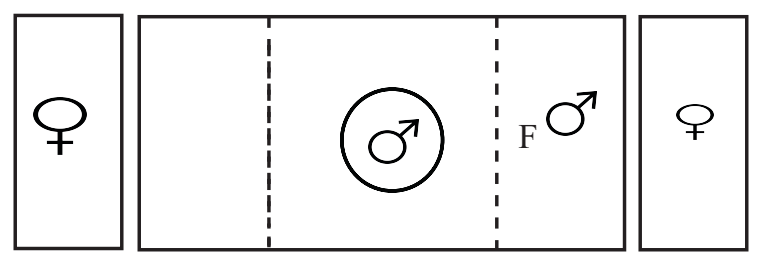

Figure 7-1 Schematic top view of the experimental apparatuses used. The central compartment $(40 \times 20 \times 25 \mathrm{~cm} ; \mathrm{L} \times \mathrm{W} \times \mathrm{H})$ held the focal male (F) and the audience male (depending on the preference test) and the end compartments $(6 \times 20 \times 22 \mathrm{~cm})$ held the stimulus females. In panel $(b)$, the central compartment concurrently held the freeswimming focal male and the 'audience' male (constrained in a clear Plexiglas cylinder, $7 \mathrm{~cm}$ diam.) during Preference Test 2. 
Table 7-1 Mean \pm SE (range) standard body length $(\mathrm{mm})$ and body ornamentation score $(\%$ of male body covered by orange and black) of guppies used in each treatment separately. Results of paired $t$-tests for homogeneity of body length and colour score among male types, and results of paired $t$-tests comparing the body length of paired stimulus females within and between treatments, are shown.

\begin{tabular}{|c|c|c|c|c|c|c|}
\hline \multirow[b]{2}{*}{ Treatment } & \multicolumn{2}{|c|}{ Male length (mm) } & \multicolumn{2}{|c|}{ Male colour score $(\%)$} & \multicolumn{2}{|c|}{ Stimulus female length (mm) } \\
\hline & Focal & Audience & Focal & Audience & Large female & Small female \\
\hline \multirow{2}{*}{$\begin{array}{c}1 \\
\text { Audience male } \\
\text { smaller and less } \\
\text { ornamented }\end{array}$} & $\begin{array}{c}19.24 \pm 0.18 \\
(16.62-21.71)\end{array}$ & $\begin{array}{c}17.61 \pm 0.14 \\
(15.47-20.12)\end{array}$ & $\begin{array}{c}7.78 \pm 0.31 \\
(4.3-13.89)\end{array}$ & $\begin{array}{c}3.93 \pm 0.36 \\
(0-9.08)\end{array}$ & $\begin{array}{c}26.72 \pm 0.32 \\
(22.55-34.07)\end{array}$ & $\begin{array}{c}21.8 \pm 0.29 \\
(17.55-26.42)\end{array}$ \\
\hline & \multicolumn{2}{|c|}{$t_{46}=9.95, P<0.001$} & \multicolumn{2}{|c|}{$t_{46}=10.37, P<0.001$} & \multicolumn{2}{|c|}{$t_{46}=19.18, P<0.001$} \\
\hline \multirow{2}{*}{$\begin{array}{c}2 \\
\text { Audience male } \\
\text { smaller and more } \\
\text { ornamented }\end{array}$} & $\begin{array}{c}19.51 \pm 0.31 \\
(16.77-23.89)\end{array}$ & $\begin{array}{c}17.41 \pm 0.24 \\
(15.06-19.36)\end{array}$ & $\begin{array}{l}4.94 \pm 0.37 \\
(1.38-7.5)\end{array}$ & $\begin{array}{c}7.08 \pm 0.41 \\
(2.26-10.12)\end{array}$ & $\begin{array}{c}27.40 \pm 0.64 \\
(22.98-36.16)\end{array}$ & $\begin{array}{c}22.64 \pm 0.61 \\
(16.9-32.18)\end{array}$ \\
\hline & \multicolumn{2}{|c|}{$t_{26}=7.97, P<0.001$} & \multicolumn{2}{|c|}{$t_{26}=-7.13, P<0.001$} & \multicolumn{2}{|c|}{$t_{26}=13.04, P<0.001$} \\
\hline \multirow{2}{*}{$\begin{array}{c}3 \\
\text { Audience male } \\
\text { larger and less } \\
\text { ornamented } \\
\end{array}$} & $\begin{array}{c}17.61 \pm 0.09 \\
(16.24-18.88)\end{array}$ & $\begin{array}{c}19.12 \pm 0.15 \\
(17.51-22.99)\end{array}$ & $\begin{array}{c}7.87 \pm 0.32 \\
(3.79-15.09)\end{array}$ & $\begin{array}{c}4.8 \pm 0.3 \\
(0.99-11.66)\end{array}$ & $\begin{array}{c}26.47 \pm 0.32 \\
(22.51-34.03)\end{array}$ & $\begin{array}{c}22.16 \pm 0.32 \\
(18.62-32.31)\end{array}$ \\
\hline & \multicolumn{2}{|c|}{$t_{49}=-9.79, P<0.001$} & \multicolumn{2}{|c|}{$t_{49}=9.04, P<0.001$} & \multicolumn{2}{|c|}{$t_{49}=17.67, P<0.001$} \\
\hline \multirow{2}{*}{$\begin{array}{c}4 \\
\text { Audience male } \\
\text { larger and more } \\
\text { ornamented }\end{array}$} & $\begin{array}{c}17.7 \pm 0.15 \\
(15.86-19.95)\end{array}$ & $\begin{array}{c}19.06 \pm 0.16 \\
(17.50-23.55)\end{array}$ & $\begin{array}{c}4.47 \pm 0.3 \\
(0.59-8.1)\end{array}$ & $\begin{array}{c}7.26 \pm 0.32 \\
(2.33-11.46)\end{array}$ & $\begin{array}{c}26.76 \pm 0.37 \\
(18.94-32.83)\end{array}$ & $\begin{array}{c}22.5 \pm 0.29 \\
(17.11-26.77)\end{array}$ \\
\hline & \multicolumn{2}{|c|}{$t_{40}=-8.25, P<0.001$} & \multicolumn{2}{|c|}{$t_{40}=-7.53, P<0.001$} & \multicolumn{2}{|c|}{$t_{40}=11.98, P<0.001$} \\
\hline
\end{tabular}


My protocol testing for an audience effect generally followed that of Auld and Godin (2015) and Chapter 6. Briefly, for all treatments, a given trial consisted of two consecutive 20-min testing phases: (i) an initial mating preference test (Preference Test 1) to establish the baseline preference of the focal male in the absence of any audience, and (ii) a second preference test (Preference Test 2) with an audience male present (Figure 71) to test for an audience effect. Therefore, each focal male was tested twice for his mating preference (i.e. in absence vs. presence of one audience male). I did not include a no-audience treatment in this experimental design, because I had previously used such a control treatment in a related experiment with similar apparatus and procedures (Auld and Godin 2015; Chapter 6) to demonstrate that wild-caught Trinidadian male guppies from my current study population are consistent (over time) in their mating preferences between two consecutive preference tests in the absence of an audience male. Similarly, in the absence of sexual competitors, I previously showed that the mating preferences of individual male guppies for larger females in this same population are consistent and highly repeatable (Godin and Auld 2013; Chapter 4).

All four treatments shared a common procedure as follows. A mate choice trial consisted of initially placing a focal male into a clear Plexiglas cylinder ( $7 \mathrm{~cm}$ diam.) located in the centre of the test aquarium and randomly placing one stimulus female into each of the two end compartments of the apparatus (Figure 7-1a). Paired stimulus females were chosen to differ in standard body length by $17.2 \pm 0.005 \%$ overall (mean \pm SE, see Table 7-1) to promote male discrimination between the females and to elicit a choice from the male (Dugatkin and Godin 1993; Jeswiet et al. 2011b; Godin and Auld 2013; Chapter 4), but matched as closely as possible for their degree of abdominal 
distension (assessed by eye) to control for stage of pregnancy (Houde 1997). I alternated between trials whether the large female was initially placed in the left or right end compartment.

The fish were left undisturbed for a 10-min acclimatization period, during which opaque screens prevented visual contact between the focal male and the stimulus females. Following acclimatization, I removed the opaque screens and allowed the focal male (constrained in the clear central cylinder) to observe the stimulus females in their respective end compartments for $10 \mathrm{~min}$ (viewing period). After this period, I released the focal male from the central cylinder and allowed him to 'choose' between the two stimulus females for 10 min. Following Herdman et al. (2004), immediately following this first 10-min test, I replaced the opaque screens, gently dipnetted the focal male and placed him into the central cylinder. I then switched the positions of the two end compartments to control for any potential side bias in the male's preference behaviour. After 5 min of acclimatization, I removed the screens, released the male from the cylinder, and scored his mating preference for a second 10-min period. Thus, the mating preference of the focal male was quantified over a total of $20 \min$ (i.e. Preference Test 1) and expressed as the proportion of his total association time (summed over the paired stimulus females) that he spent near either of the females. Trials in which focal males exhibited a side bias were discarded (see Chapter 2 for details on side biases). The preferred stimulus female was considered the one with whom the focal male spent more than $50 \%$ of his total association time. This criterion, or slight variants of it, for mating preference is commonly used for poeciliid fishes (e.g. Bisazza et al. 1989; Plath et al. 2008c; Jeswiet et al. 2011b) and is a strong predictor of mating effort and mate choice in 
the guppy (Dugatkin and Godin 1992; Jeswiet and Godin 2011) and other species (e.g. Witte 1996b, Lehtonen and Lindström 2008; Walling et al. 2010).

Immediately following Preference Test 1, I replaced the opaque screens and placed the focal male in the central cylinder. I then introduced an audience male of a known body length and colour phenotype combination (depending on the treatment, Table 7-1) into another clear Plexiglas cylinder (7 $\mathrm{cm}$ diam.) adjacent to the focal male cylinder in the centre of the test aquarium and equidistant between the two stimulus females (Figure 7-1b). Following a 5-min acclimatization period, I removed the opaque screens and allowed the focal male (whilst still in the cylinder) to view the stimulus females and the audience male for $5 \mathrm{~min}$. At the end of this viewing period, I released the focal male from his cylinder and recorded his mating preference for another $20 \mathrm{~min}$ in the presence of an audience of one rival male (i.e. Preference Test 2). The audience male remained in his cylinder throughout this mating preference test. Preference Test 2 was carried out exactly the same as Preference Test 1, except for the inclusion an audience male. I quantified the mating preference of the focal male for either of the paired stimulus females as described for Preference Test 1.

Trials for the four audience treatments were carried out in random order. I tested 47, 27, 50 and 41 focal males (that showed no side bias) in Treatments 1, 2, 3 and 4, respectively, for a total of 165 trials (Table 7-1). I used different audience males and stimulus females for each trial. None of the fish were re-used. 


\subsubsection{Statistical analysis}

All $t$-tests used were two-tailed and all statistical analyses were carried out in the $\mathrm{R}$ statistical framework (R Core Team 2014).

I first tested whether focal males exhibited an overall initial mating preference for the larger stimulus female in Preference Test 1 across all four treatments by comparing the observed proportion of their total association time spent near the larger female against that expected by chance (i.e. 50\%) using a one-sample $t$-test, and complementarily comparing the number of males categorized as preferring the large females versus that preferring the smaller female using the $G$-test (Signorell et al. 2015). Second, to ascertain whether males modified their initial mating preference in the presence of an audience, I compared the proportion of total association time individual focal males spent with their initially-preferred female in Preference Test 1 (no audience) to that in Preference Test 2 (audience present) using the paired $t$-test for each treatment separately. Third, to compare the magnitude of any observed audience effect across treatments, I calculated for each focal male a difference score for any change in mating preference between the matched preference tests $[$ Difference score $=($ Proportion of time near the initially-preferred female in Preference Test 2 - Proportion of time near the initially non-preferred female in Preference Test 1)], following Auld and Godin (2015) and Chapter 6. A negative value here denotes a decrease in the proportion of time a focal male spent with the initiallypreferred female, and thus an increased relative preference for the other female, in the second preference test during the presence of an audience male (taken as evidence for an audience effect). 
I then used a linear model to test for potential effects of treatment and the strength of the mating preference of focal males for their initially-preferred female (in Preference Test 1), and their interaction, on the magnitude of the audience effect (as measured by the difference score of individual focal males). I also included in the model the standard body length and colour score of focal males as covariates. In a post-hoc analysis of the model's fitted values, I compared mean difference scores among treatments using a one-way ANOVA to control for differences attributable to an interaction effect between treatment and the proportion of time males spent with their initially-preferred female, followed by paired $t$-tests to test for pair-wise differences in the magnitude of the audience effect between treatments. I used the Benjamini-Hochberg method (Benjamini and Hochberg 1995) to control for false discovery rate related to the multiple paired $t$-tests. Lastly, I correlated each focal male's difference score with his initial mating preference score (for Preference Test 1), and calculated confidence intervals for the correlation coefficient, using the R package 'compute.es' (Del Re 2013) for each treatment separately. Residuals from the models were normally distributed, as determined from Q-Q plots.

\subsubsection{Ethical standards}

The current study was approved by the institutional Animal Care Committee at Carleton University (protocol \#12157) and thus meets the guidelines for the care and use of research animals of the Animal Behavior Society and the Canadian Council on Animal Care and the laws of Canada. 


\subsection{Results}

Male guppies exhibited strong initial mating preferences for one or the other of the paired stimulus females in Preference Test 1 (Figure 7-2a). Overall across all treatments, focal males spent a greater proportion of their total association time with the larger of the paired females (mean $\pm \mathrm{SE}=0.55 \pm 0.02)$ in Preference Test 1 than would be expected by chance (one-sample $t$-test: $t_{164}=2.67, P<0.008$ ), and correspondingly significantly more males (95 out of 165) categorically preferred the large over the smaller female than expected by chance ( $G$-test: $\left.G_{a d j 1}=3.80, P=0.05\right)$, thereby demonstrating an overall initial baseline male mating preference for the larger female as expected a priori.

Males reduced, but not significantly so (paired $t$-test: $t_{26}=1.83, P=0.08$; Figure 7-2a), their initial mating preference for a particular female while being observed by a sexual rival who was smaller and more ornamented than themselves (Treatment 2). For the other three treatments in comparison, males did significantly reduce their preference for their initially-preferred female in the presence of an audience male who was either smaller and less ornamented $\left(t_{46}=2.95, P=0.005\right)$, larger and more ornamented $\left(t_{40}=\right.$ $3.17, P=0.003)$, or larger and less ornamented $\left(t_{49}=6.15, P<0.001\right)$ than themselves. So a strong audience effect was observed in three of the four treatments (Figure 7-2).

As expected, the magnitude of any observed change in a male's preference for a given female in Preference Test 2 compared to his preference for that same female in Preference Test 1 (= difference score) varied among audience treatments (linear model: $F_{3,161}=2.92, P=0.04$; Figure $7-2 b$ ). There was no significant effect of the body length (linear model: $F_{3,161}=0.46, P=0.50$ ) or colour score (linear model: $F_{3,161}=0.37, P=$ 0.84) of focal males on their difference scores. However, there was a significant effect of 
the initial mating preference score of focal males (linear model: $F_{3,161}=21.11, P<$ 0.001), and a significant interaction between the latter variable and the phenotype of the audience male (linear model: $F_{3,161}=3.37, P=0.02$ ), on observed difference scores. Because of this statistical interaction, I controlled for differences in the initial strength of male mating preferences among treatments by running an ANOVA on the linear model's fitted values, which confirmed that observed difference scores (= magnitude of audience effect) varied significantly among treatments (one-way ANOVA: $F_{3,161}=14.45, P<$ 0.001; Figure 7-2b). Post-hoc pairwise comparisons of mean difference scores (Figure 7$2 b$ ) revealed that focal males reduced their initial preference for a given female to a significantly greater degree when the audience male was relatively larger than themselves (Treatments 3 and 4) compared to when the audience male was smaller (Treatments 1 and 2), irrespective of whether the audience was more or less colour ornamented.

Surprisingly, the magnitude of the audience effect was greater $(P=0.03)$ when the audience male was smaller and less colour ornamented than the focal male (Treatment 1) compared to when the audience was smaller and more colour ornamented (Treatment 2). There was no significant difference $(P=0.08)$ in the magnitude of the audience effect between Treatments 3 and 4 (Figure 7-2b).

The magnitude of the audience effect (i.e. difference scores) did not depend on whether the focal male initially preferred the larger or smaller of the paired stimulus females $\left(t\right.$-test: $\left.t_{1,163}=0.09, P=0.76\right)$. However, the difference scores of individual focal males depended on the strength of their initial mating preference for a particular female in Preference Test 1 (Figure 7-3). When the audience was relatively larger [Treatment 3 (correlation: $r=-0.41, P=0.003$ ) and Treatment 4 (correlation: $r=-0.53, P<0.001$ )], 
this relationship was significantly negative (Figure 7-3); that is, the stronger the initial mating preference of a focal male for a particular female (in Preference Test 1), the more he subsequently reduced his preference for that same female in the presence of an audience male (i.e. the greater the difference score). There was no significant relationship when the audience male was relatively smaller than the focal [Treatment 1 (correlation: $r$ $=-0.1, P=0.49$ ) and Treatment 2 (correlation: $r=-0.34, P=0.08)]$. The slope of the relationship was greatest when the audience males were larger than the focal males, irrespective of the relative differences in their colour scores (Treatments 3 and 4). 


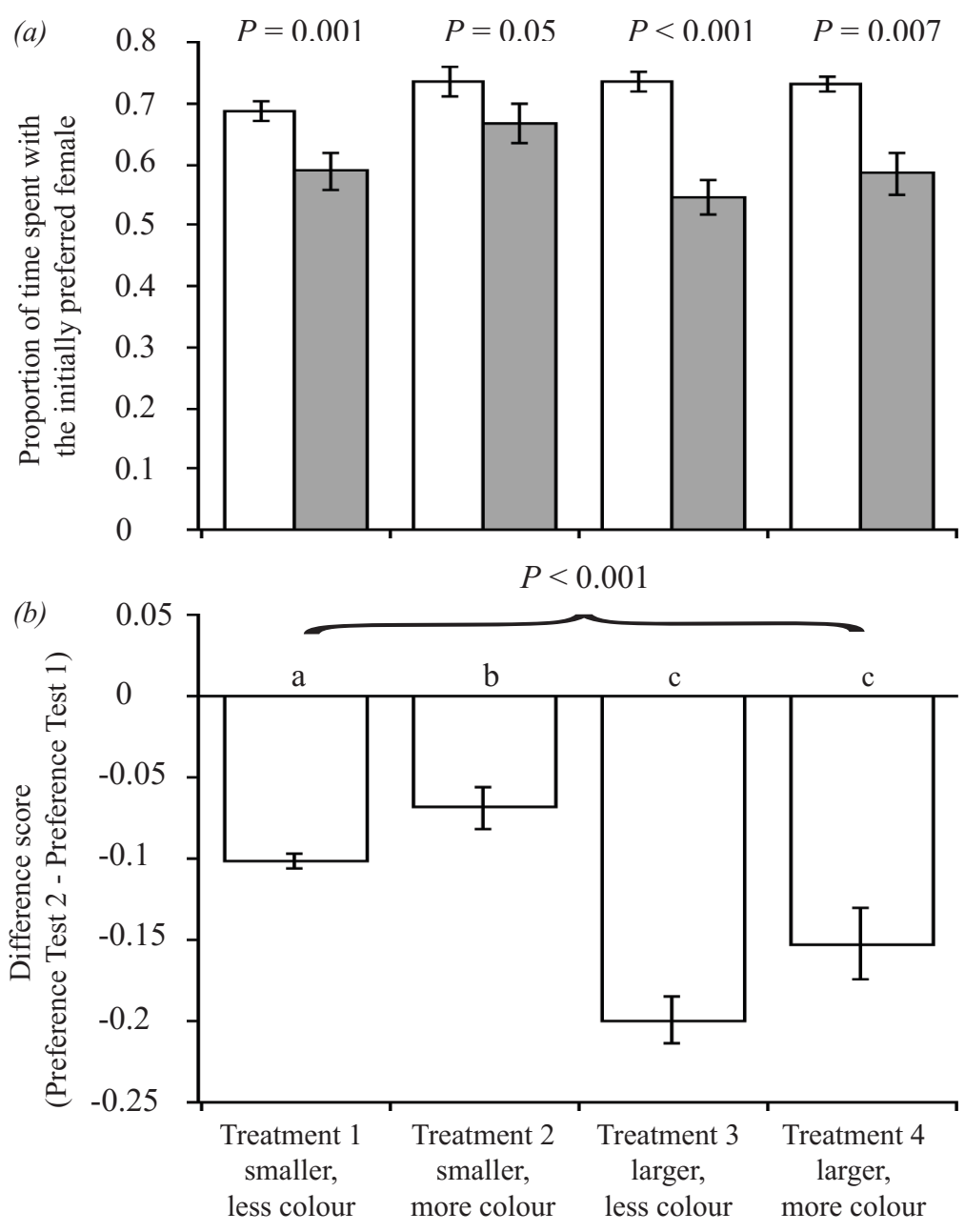

Figure 7-2 Comparison of the initial mating preference of focal males for one of the two stimulus females presented in the absence of an audience male (Preference Test 1) compared to their preference for the same female in the presence of an eavesdropping audience male (Preference Test 2), who was either smaller and less colour ornamented (Treatment 1), smaller and more ornamented (Treatment 2), larger and less ornamented (Treatment 3) or larger and more ornamented (Treatment 4 ) than the focal male. The mating preferences of focal males are shown using two measures: $(a)$ mean $\pm \mathrm{SE}$ proportion of time that focal males spent associating with the initially-preferred female in Preference Test 1 (open bar) and Preference Test 2 (filled bar); $(b)$ mean $( \pm \mathrm{SE}$ ) preference difference score between Preference Test 2 and Preference Test 1 (a negative value denotes a decrease in the focal male's preference for the initially-preferred female in Preference Test 2 calculated from linear model fitted values). Histogram bars with dissimilar letters are significantly different $(P<0.05)$ from each other. 


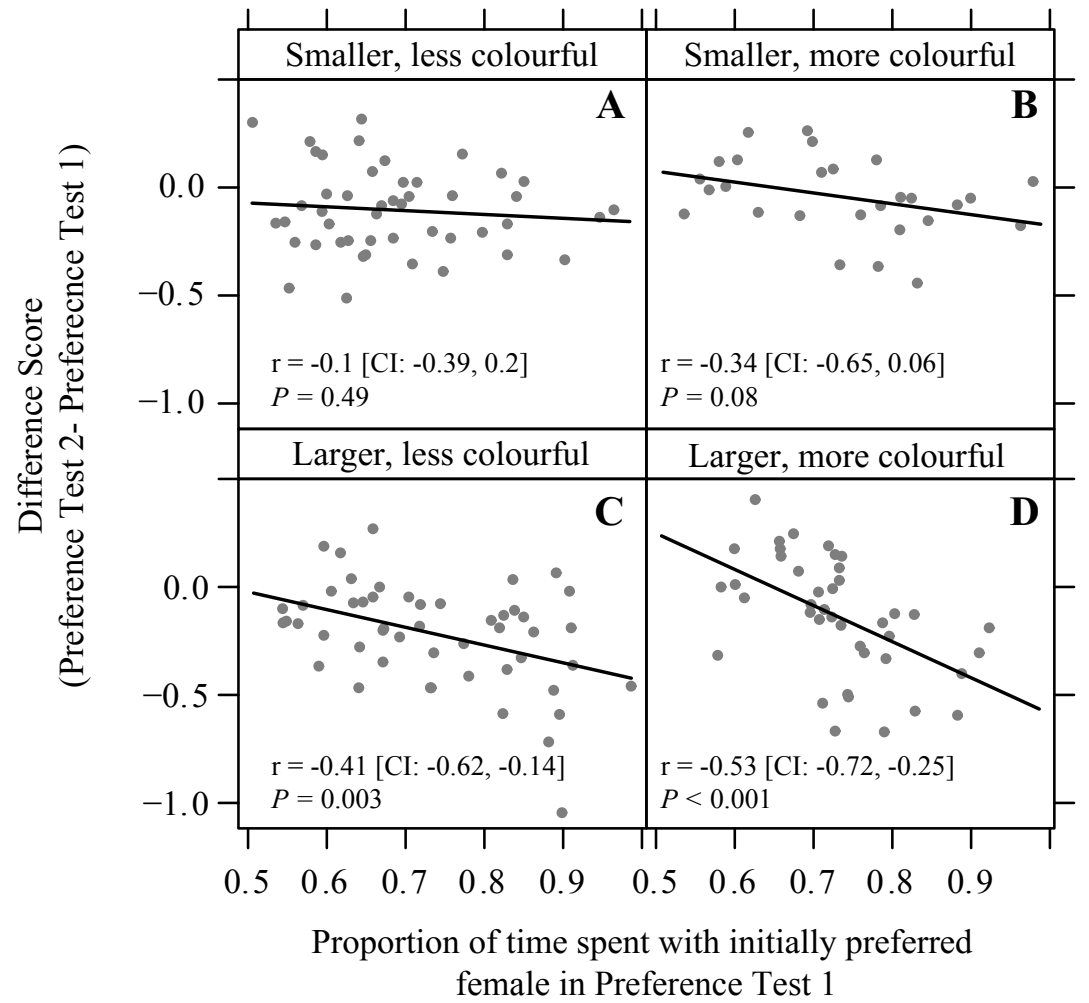

Figure 7-3 Relationship between the initial mating preference of focal males for a particular female (in Preference Test 1) and the change in their mating preference for the initially-preferred female in the presence of an audience male (Preference Test 2) who was either smaller and less colour ornamented (Treatment 1), smaller and more ornamented (Treatment 2), larger and less ornamented (Treatment 3 ) or larger and more ornamented (Treatment 4) than themselves. The arcsine-transformed partial residuals from the linear model are plotted. In each panel, the best-fit regression line and the correlation coefficient (with confidence intervals) of the relationship are shown. 


\subsection{Discussion}

In mating systems wherein males compete for mating access to a limited number of sexually-receptive females that vary widely in reproductive value and can copy the observed mating preferences of other males (i.e. demonstrators), and some degree of lastmale sperm precedence and sperm competition exist, as is the case for the Trinidadian guppy (Houde 1997; Kelly et al. 1999; Evans and Magurran 2001; Neff and Wahl 2004; Neff et al. 2008; Jeswiet et al. 2011b; Auld and Godin 2015; Chapter 5), demonstrators could benefit from flexibly adjusting their mating behaviour so as to withhold or reduce social information about their mating preferences or mate choice in the presence of an audience of one or more eavesdropping sexual rivals (Nordell and Valone 1998; Dubois 2015; Castellano et al. 2016). A known audience-mediated evasive tactic is a reduction in the initial mating preference of a focal (demonstrator) male for a particular female, and an increase in his sexual attention towards another previously non-preferred female, in the presence of an eavesdropping audience male (e.g. Dzieweczynski et al. 2005; Plath et al. 2008a,b, 2009; Ziege et al. 2008, 2009; Dzieweczynski and Walsh 2010; Mautz and

Jennions 2011; Bierbach et al. 2013; Auld et al. 2015; Auld and Godin 2015; Chapters 6 and 8).

Here, I showed that wild-caught male guppies from a low-predation population (Upper Aripo River) in Trinidad exhibited non-random preferences for either of two potential female partners that varied in body size (and thus potential fecundity) and expressed an overall initial mating preference for the larger female, even though considerable inter-individual differences in preference were observed. These results are as expected and corroborate previous studies on male mate choice in Trinidadian guppies 
originating from different natural populations, including my current study population (Dosen and Montgomerie 2004a; Herdman et al. 2004; Jeswiet et al. 2012; Godin and Auld 2013; Auld and Godin 2015; Chapters 4 - 6). In the mere presence of an audience sexual rival, male guppies on average reduced their initial preference for a particular female, and correspondingly increased their association time spend near the other previously non-preferred female, as expected; that is, they exhibited an overall audience effect on mating preferences. Similar audience-mediated adjustment in male mating preferences have been previously observed in the Trinidadian guppy (Makowicz et al. 2010b; Auld and Godin 2015; Chapter 6) and other poeciliid fishes (e.g. Plath et al. 2008a, b, c, 2009; Makowicz et al. 2010a; Bierbach et al. 2011a; but see Bierbach et al. 2012 for an exception).

Notwithstanding the above validating findings, the most novel results of my current study are that $(i)$ focal male guppies responded to the presence of a nearby audience male by reducing their initial mating preference for a particular female in a graded manner, which depended on the relative differences in the expected sexual attractiveness and competitiveness of the audience and focal males (as measured by their respective body length and body ornamentation), and (ii) the magnitude of a focal male's response to an audience sexual rival (i.e. the audience effect) was positively correlated with the strength of his initial mating preference. Male guppies were therefore sensitive to the phenotype of a nearby audience male and reduced their initial preference for a particular mate to a greater degree in the presence of a relatively larger audience male compared to a relatively smaller one, irrespective of differences in their body ornamentation. With the exception of Bierbach et al.'s (2011b) recent study and my 
current study, such phenotype-dependent audience effects on male mating preferences have hitherto not been investigated empirically. Therefore, my results above are particularly novel, important and of broad theoretical interest (Dubois 2015; Costellano et al. 2016).

In species with male mate-choice copying, intense sperm competition and some degree of last-male sperm precedence, such as the guppy (refs. loc. cited; Chapter 5), an audience-mediated attenuation of the initial mating preferences of males for one of two potential mates (and a corresponding increase in mating effort directed towards a previously non-preferred female partner; Chapter 6 and current study) could be adaptive if it either mitigates the costs associated with direct, pre-copulatory male-male sexual competition for a preferred female ("flexible decision hypothesis", Mautz and Jennions 2011; Costellano et al. 2016) or prevents eavesdropping audience males from copying the mate choice of focal males, thereby minimizing their risk of sperm competition, by deceptively redirecting the attention of audience male rivals away from the focal males' preferred female by reducing the amount of inadvertent public information about the latter males' true mating preference that is available to eavesdroppers (“deception hypothesis", Plath et al. 2008c; Bierbach et al. 2013; Dubois 2015; Castellano et al. 2016). These two functional hypotheses are not necessarily mutually exclusive in my opinion. Of these hypotheses, I argue below that the deception hypothesis better explains my results overall.

Focal male guppies in the current study reduced their initial mating preference to a greater extent when the audience male was relatively larger than themselves compared to when the audience male was relatively smaller, irrespective of relative differences in 
their body ornamentation. Because of the potential fitness costs associated with being copied (i.e. elevated risk of sperm competition) and the risk of being subsequently rejected by their initially-preferred females, selection should favour males who strategically adjust their mating preference in the presence of more sexually attractive and competitive audience rivals. Larger audience males are likely to be perceived as stronger sexual and sperm competitors by focal males because they are more attractive to females (Auld et al. 2016; Chapter 3), produce larger quantities of sperm (Skinner and Watt 2007) and are better burst accelerators and swimmers (Odell et al. 2003), and thus perhaps better at sneak copulations, than smaller males. If focal males were responding to a perceived risk of pre-copulatory sexual competition (flexible decision hypothesis), then I would expect a stronger audience effect when focal males initially preferred the larger (and potentially more fecund) of the two stimulus females, a preference which most males have in my study population (Godin and Auld 2013; Auld and Godin 2015; Chapters 4 - 6; current study). On the contrary, the magnitude of the audience effect did not depend on whether focal males initially preferred the larger or smaller of the paired stimulus females. Moreover, direct and actual pre-copulatory male-male sexual competition was not possible in my study because the audience male remained constrained in a clear cylinder in the centre of the test aquarium, and thus could not physically interact with the focal male, throughout the mate choice trial. Direct physical aggression among male guppies in the wild is relatively rare and does not appear to be a significant determinant of male mating success (Houde 1997).

Similar to my evidence for a phenotype-dependent audience effect, Bierbach et al. (2011b) found that, in the presence of an audience of potential sexual rivals, male 
Atlantic mollies (Poecilia mexicana) exhibited a greater reduction in their initial mating preference when the audience male was larger and unfamiliar, or larger and known to be sexually active. Because focal and audience male guppies used in my current study were selected from different aquaria and were presumably unfamiliar with each other (see Chapter 2 for details), it is unlikely that either social familiarity or a prior knowledge of the sexual experience of rival audience males could have influenced the magnitude of the audience effect observed in my study.

Contrary to my a priori prediction, focal males unexpectedly decreased their preference for their initially-preferred female to a greater extent when the audience male was relatively less colour ornamented than the focal male, irrespective of the relative differences in their body length. Female guppies prefer males with a greater proportion of their body covered with orange and black pigmentation (Auld et al 2016; Chapter 3; but see Schwartz and Hendry 2010) and males with more orange coloration produce faster and more viable sperm (Locatello et al. 2007) in my study population, and male guppies with more orange colour in general produce more sperm (Pitcher and Evans 2001; Pitcher et al. 2007) and have greater insemination success (Pilastro et al. 2002) than less ornamented males, suggesting that males with greater colour ornamentation should be perceived as stronger sexual and sperm competitors.

Interestingly, individual males that had an initially strong initial mating preference for a particular female exhibited a stronger audience effect (i.e. decreased their initial preference for the initially preferred female to a greater extent) than males with weaker initial mating preferences. This result is consistent with the deception hypothesis as it suggests that the more highly a focal male values a particular female partner, the 
more willing he is to invest in deceptive social information that redirects the attention of a nearby audience male away from his preferred mate, so as to minimize the risks of being copied and incurring sperm competition, but at the potential cost of being subsequently ignored by his initially preferred female (lost mating opportunity cost) once the deceived audience male is no longer an immediate threat. In support of this proposition, Bierbach et al. (2013) showed that the audience effect on male mating preferences is greatest in poeciliid fish species in which males exhibit the highest level of sexual activity, suggesting that sperm competition likely drives such audience effect. Contrary to my recent study (Auld and Godin 2015; Chapter 5) demonstrating matechoice copying in wild-caught Trinidadian male guppies, Makowicz et al. (2010b) found that male guppies decreased their mating preference for a given female in a no-choice test in the presence of an audience compared to when no audience was present, but found no evidence that audience males subsequently copied the mate choice of the (demonstrator) male they had previously viewed mate with a female. They concluded that the risk of mate-choice copying could not be responsible for the observed audience effect in their study. Differences between their experimental design and fish provenance and mine (see details in Auld and Godin 2015; Chapter 5) likely account for differences in my respective findings on mate-choice copying and conclusions on the importance of sperm competition risk in the evolution of male evasive responses to a rival male audience.

Residual variation in the direction and strength of change in a male's initial mating preference in the presence of a rival male audience not explained by relative differences in the phenotypes of focal and audience males may be attributable to interindividual differences in their personality and(or) past experiences. For example, in 
another poeciliid species (the Atlantic molly), Bierbach et al. (2015) found that under high sexual competition bolder males reduced their initial mating preference for a particular female to a greater extent than did shy males. I did not take into account potential differences in personality between male guppies in my study. Whether audience-mediated changes in the mating preferences of male guppies depend partly on their personality, as measured for example by their boldness level, is unknown and should be tested in future, given that boldness and colour ornamentation are positively correlated in male guppies originating from a high-predation population in Trinidad and that females prefer bold males as mates (Godin and Dugatkin 1996).

Overall, my results add to a growing body of evidence showing that males are sensitive to their social environment when making mating decisions and respond in a manner which presumably increases their individual reproductive success. Here, I showed that male Trinidadian guppies strategically respond to the mere presence of eavesdropping rival males based on relative differences in phenotypic traits (body length and ornamentation) that are known indicators of sexual attractiveness and competitiveness. My results further highlight the importance of social information in mediating the mating decisions of males, and thus sexual selection, in polygamous species.

\subsection{Acknowledgments}

I thank the Director of Fisheries of Trinidad and Tobago for permission to collect fish from the Upper Aripo River, G.E. Brown, C. Elvidge, B. Daniel, R. Mahibir and K. 
Deonaransingh for assistance with fish collection, and A. Miller and C. Elvidge for assistance with data collection. 


\section{Chapter 8:}

\section{Do Male Trinidadian Guppies Adjust their Alternative Mating Tactics in the Presence of a Rival Male Audience?}

This chapter is published as: Auld, H.L., Jeswiet, S.B. and Godin, J.-G.J. 2015. Do male Trinidadian guppies adjust their alternative mating tactics in the presence of a rival male audience? Behavioral Ecology and Sociobiology, 69: 1191-1199. It is reproduced here with permission from Springer-Verlag Berlin Heidelberg ${ }^{\mathcal{O}}$. 


\subsection{Abstract}

Males in polygamous mating systems may inadvertently transmit information regarding their mating preferences to bystanding sexual competitors, thereby permitting bystanders to use this information to enhance their own mating success by copying the mate choice of signallers. If males are at risk of having their mate choice copied and consequently face a higher risk of sexual competition, then selection should favour males that reduce conspicuous mating behaviours in the presence of an audience of sexual competitors. In the current study, I used the Trinidadian guppy (Poecilia reticulata), a species that exhibits alternative male mating tactics, to test this sexual competition avoidance hypothesis and the predictions that males would decrease their overall mating effort and exhibit fewer conspicuous courtship displays relative to more inconspicuous sneak mating attempts in the presence of either one or two sexual rivals compared to the absence of any audience. Male guppies significantly reduced their overall mating effort in the presence of increasing numbers of rival audience males. This was reflected in similar monotonic decreases in the frequencies of courtship displays and sneak mating attempts and in the proportional use of courtship displays (relative to sneak mating attempts) across treatments. These findings are consistent with the sexual competition avoidance hypothesis. My novel results contribute to an increasing body of knowledge showing that the social environment can influence the mating effort and mating decisions of individuals and thus have important implications for sexual selection and evolution. 


\subsection{Introduction}

The social environment can importantly influence the behaviour of socially-interacting animals and thereby selection and social evolution (Moore et al. 1997; Westneat et al. 2000; McGlothlin et al. 2010). In polygamous mating systems in particular, sexuallyinteracting individuals are commonly within view and signal-receiving distances of other conspecifics, thus forming communication networks (McGregor and Peake 2000). Whilst interacting with potential mates and sexual rivals in such a social context, a male may inadvertently or purposefully transmit to other males social information (sensu Danchin et al. 2004) about his mating preference and (or) his own quality. Nearby bystanding or eavesdropping males (= the 'audience', sensu McGregor and Peake 2000) observing such interactions would receive information about the quality of potential mates and (or) the competitiveness of rivals and subsequently might use this information in making their own mating decisions (Westneat et al. 2000; Danchin et al. 2004; Dabelsteen 2005; Valone 2007). Such social eavesdropping can be beneficial for the bystander as it provides a relatively low-cost, low-risk alternative to gathering the same socio-sexual information acquired through direct interaction with the individual being observed (Dabelsteen 2005). For the individual being eavesdropped upon, however, the opposite may be true (Dabelsteen 2005). For example, female fighting fish (Betta splendens), which had previously witnessed a male-male aggressive interaction, subsequently preferred as a mate the winner of the interaction over the loser, whereas females that did not witness the aggressive interaction showed no bias towards either the winner or loser male (Herb et al. 2003). In this case, the presence of a female audience was beneficial for the winner of the fight, but detrimental for the loser. 
Under certain circumstances, males will alter their aggressive behaviour and (or) mating preference in the mere presence of an audience, a response referred to as the audience effect (sensu Dabelsteen 2005). Such audience-mediated behavioural plasticity has been reported for several vertebrate species, including fishes (Dzieweczynski et al. 2005; Plath et al. 2008a,b,c, 2009; Ziege et al. 2008; Makowicz et al. 2010a, b; Chapters 6-7), birds (Evans and Marler 1994; Striedter et al. 2003; Dubois and Belzile 2012), and mammals (Pollick et al. 2005).

In recent years, increasing attention has been directed towards the audience effect within the sexual context. For instance, males in some fish species (e.g. Atlantic molly, Poecilia mexicana (Ziege et al. 2009), Grijalva mosquitofish, Heterophallus milleri (Ziege et al. 2008), threespine stickleback, Gasterosteus aculeatus (Dzieweczynski et al. 2005), guppy (Chapters 6 - 7) exhibit weaker mating preferences in the presence of a conspecific male audience. Similarily, male pacific blue eye (Pseudomugil signifier) reduce their courtship behaviour when visually interacting with another conspecific male (Wong 2004). If a male is seen courting and successfully attracting a female, then this may reveal to a competing (audience) male information about the female's receptivity and willingness to mate (Schlupp and Ryan 1997) and thereby potentially increase the level of sexual competition and risk of sperm competition for the signalling focal male (Jeswiet et al. 2011b). In reducing his courtship behaviour towards, and sexual preference for, a particular preferred female in response to a rival male audience, a focal male may be either concealing his true sexual interest in that female or deceiving his sexual rivals by diverting their sexual attention away from his preferred female (Plath et al. 2008b, c), thereby potentially minimizing his risk of sexual competition (Plath and Schlupp 2008; 
Ziege et al. 2009; Jeswiet et al. 2011b, 2012) and possibly avoiding rejection by the preferred female (Plath and Schlupp 2008; Dubois and Belzile 2012).

In addition to flexibly adjusting their courtship behaviour and mating preferences (refs. loc. cited), males may also flexibly modify their relative use of alternative mating tactics (Gross 1996; Brockmann 2001) in response to an audience of sexual competitors. Alternative male mating tactics are common in highly competitive sexual environments in nature (Gross 1996; Brockmann 2001), and their relative frequencies of use can be plastic and dependent on an individual male's phenotype and social status (Howard 1984; Jirotkul 2000b; Ortega and Arita 2002), ecological factors (Godin 1995; Rodd and Sokolowski 1995), and social factors such as conspecific density (Jirotkul 1999b) and operational sex ratio (Jirotkul 1999a, 2000a), for example. Two of the most common male mating tactics in animals are courtship displays, used to attract and influence females to mate consensually, and sneak or coerced copulations/matings, used to circumvent dominant males and (or) pre-copulatory female choice (Andersson 1994; Gross 1996; Brockmann 2001). Courtship displays are usually more prolonged, energetically costly and conspicuous compared with sneak mating attempts (Houde 1997). If a courtship display reveals more inadvertent social information about a focal male's sexual interest in a particular female than a sneak mating attempt would, then selection should favour a context-dependent shift in the relative frequency of use of these two alternative mating tactics, from predominantly courting to sneak mating, by a focal male in the presence of an audience of sexual competitors. Such an audience-mediated shift in the use of alternative mating tactics would presumably minimize a focal male's likelihood of attracting the interest of rivals towards his preferred female, whilst 
concurrently still pursuing mating opportunities via less conspicuous sneaking behaviour. To date, there has been limited empirical research on the potential effect of an audience on the use of alternative mating tactics in males (see Nöbel and Witte 2013).

Here, using the Trinidadian guppy (Poecilia reticulata) as a model system, I experimentally tested the hypothesis of a context-dependent shift in the use of alternative mating tactics by males in response to the mere presence of a rival-male audience (hereafter referred to as the sexual competition avoidance hypothesis). Adult male guppies are plastic in their mating behaviour and are individually capable of exhibiting two alternative mating tactics (Houde 1997), either courtship displays or sneak (coerced) copulation attempts, which are the principle components of their mating effort (Godin and Auld 2013; Chapter 4).

Assuming that selection favours males which behave in ways that avoid attracting the attention of sexual rivals and minimize their risk of sexual competition, I predicted that (i) male guppies should perform proportionally fewer courtship displays (relative to sneak copulation attempts), and (ii) the magnitude of any audience effect should increase with increasing membership size of the audience.

\subsection{Methods}

\subsubsection{Study animals}

I used wild-caught Trinidadian guppies as my experimental subjects. Please refer to Chapter 2 for a rationale underlying my use of the guppy as a model study system and for a more detailed description of its mating system and reproductive behaviours. 


\subsubsection{General protocol}

See Chapter 2 for a description of the common general protocol.

\subsubsection{Experimental apparatus}

The experimental apparatus consisted of a clear Plexiglas test aquarium $(40 \times 20 \times 25 \mathrm{~cm}$;

L x W x H; Figure 8-1). See Chapter 2 for more information on general test aquaria conditions.

\subsubsection{Experimental protocol}

The experimental design consisted of three audience Treatments, namely, an audience of either 0,1 or 2 conspecific males presented to a focal male, and each treatment comprised two consecutive Test periods (i.e. absence vs. presence of an audience). Each focal male subject was tested repeatedly in these two (paired) tests for his mating behaviour directed towards a stimulus female, with Test 1 carried out in the absence of an audience and Test 2 carried out in the presence of a rival-male audience. Individual male subjects were assigned at random to one of the three treatments.

For any given trial, a stimulus female was randomly selected from the pool of isolated females and gently dip-netted into the test aquarium. A focal male was then selected from the pool of isolated males and temporarily placed into a clear Plexiglas cylinder $(7 \mathrm{~cm}$ diam.) located in the centre of the test aquarium. The fish were allowed to view each other for $10 \mathrm{~min}$. Following this viewing/acclimatization period, the cylinder was gently removed, allowing the male and female to interact freely with each other for $20 \min (=$ Test 1$)$. During this test period, I recorded the number of courtship displays and sneak copulation attempts directed by the male towards the female. At the end of 
Test 1, the male was dip-netted, the cylinder returned to the centre of the aquarium and the male placed into the cylinder; the female was allowed to continue freely swimming about in the experimental aquarium. After an additional 5 min acclimatization period, paired Test 2 was carried out as follows.

For Test 2, a second clear Plexiglas cylinder (= the audience cylinder) was placed in the centre of the test aquarium for the entire duration $(20 \mathrm{~min})$ of the test. For Treatment 1 , the audience cylinder remained empty as a control for any potential effect of the mere presence of the audience cylinder on focal male behaviour. For Treatment 2, the audience cylinder contained one audience male that was selected from the pool of isolated male guppies. In Treatment 3, the audience cylinder contained two audience males. It is important to note that the constrained audience males were only bystanders and could not physically interact with the focal male and stimulus female. Because female guppies generally prefer larger and (or) more colour ornamented males (Houde 1997; Herdman et al. 2004; Pilastro et al. 2004; Chapter 3) and larger and (or) more ornamented males may be better sperm competitors (Locatello et al. 2006, but see Evans et al. 2003b), the audience male(s) and the focal male were matched for standard body length (measured with a metric scale) and ornamentation as closely as possible (initially assessed by eye, and later measured from digital photographs; see Chapter 2 and Table 81). After the audience male(s) was (were) gently placed in the audience cylinder, the focal male (still in his cylinder), the audience male(s) and the free-swimming stimulus female were allowed to view one another for $10 \mathrm{~min}$. Following this viewing period, the focal male was released by gently raising his Plexiglas cylinder out of the aquarium and a second 20-min observation period (Test 2) was initiated, during which the same focal 
male behaviours were recorded as for Test 1 . Each male's baseline mating effort was always determined prior to testing his mating effort in the presence of an audience, instead of alternating the order of Test 1 and Test 2, to control for perceived sperm competition and so as to not confound baseline behaviour. I similarly tested 27, 26, and 22 different focal males in Treatments 1,2 and 3, respectively, who demonstrated sexual interest in the female in both Test 1 and Test 2 and performed both courtship displays and sneak mating attempts. 
(a) Test 1

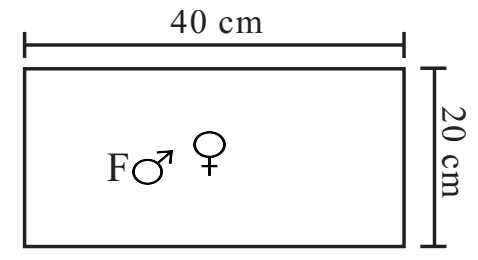

(b) Test 2

Treatment 1

No audience

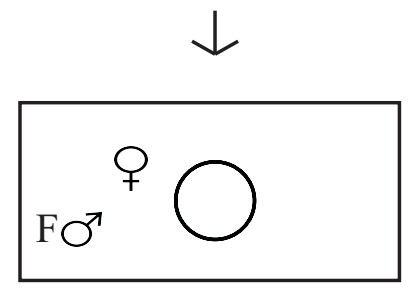

or

Treatment 2

One male

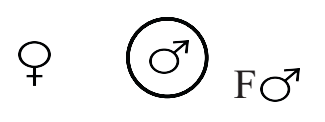

or

Treatment 3

Two males

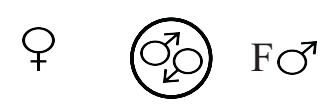

Figure 8-1 Schematic top view of the experimental apparatus. In Test 1, the test aquarium ( $40 \times 20 \times 25 \mathrm{~cm}$; L x W x H) held the focal male and stimulus female. The circle represents a removable clear Plexiglas cylinder (7 cm diam.), in which the focal male could temporarily be placed. In Test 2 , the aquarium held the focal male, the stimulus female and the 'audience' fish (constrained in a separate clear cylinder (not shown) in the centre of the aquarium throughout the test). The audience cylinder was either empty or held one or two conspecific audience males, depending on the treatment. 


\subsubsection{Statistical Analysis}

To control for differences among focal males in their initial baseline behaviour (in Test 1), I first calculated the relative change in the number of courtship displays exhibited by a focal male between Test 1 and Test 2 as [Change in display rate $=($ Frequency of courtship displays in Test 2 - Frequency of courtship displays in Test 1)/(Frequency of courtship displays in Test $1+$ Frequency of courtship displays in Test 2)]. I similarly calculated the relative change in the number of sneak mating attempts exhibited by a focal male between Test 1 and Test 2 . I then calculated the proportion of all mating attempts exhibited by a focal male that constituted courtship displays as [i.e. proportion of courtship displays $=\#$ of courtship displays $/$ (\# of courtship displays $+\#$ of sneak attempts)] in Test 1 and Test 2 for each treatment separately. Finally, I calculated the change in their proportional use of courtship displays between Test 1 and Test 2 as [change in proportional use of courtship displays $=$ (proportion of courtship displays in Test 2 - proportion of courtship displays in Test 1)/proportion of courtship displays in Test 1].

To test for an audience treatment effect on the mating effort of focal males, I used a multivariate analysis of variance (MANOVA) with relative changes in the frequencies of courtship displays and sneak mating attempts and in the proportional use of courtship displays as the dependent response variables and the audience treatment levels as the independent variable. Because the residuals of the MANOVA were not multivariate normally distributed (Royston's test, $H=14.81, P<0.001$ ), they violated one of the assumptions of the parametric MANOVA. Consequently, I necessarily carried out a nonparametric, permutational MANOVA (Anderson 2001) using the 'vegan' package 
(Oksanen et al. 2010) in the R statistical environment ( $\mathrm{R}$ Core Team 2014), with the same dependent and independent variables as mentioned above. Following this global permutational MANOVA testing for an overall audience treatment effect on male mating effort, I compared post-hoc mating effort across different pairs of treatment levels using individual MANOVAs with Bonferroni correction for multiple comparison.

\subsubsection{Ethical standards}

The current study was approved by my institutional Animal Care Committee (protocol 8557), and adheres to the animal use and care guidelines of the Canadian Council on Animal Care and thus the laws of Canada. 
Table 8-1 Mean \pm SE (range) standard body length $(\mathrm{mm})$ and body ornamentation score $(\%$ of male body covered by orange

pigmentation) of guppies used in the three audience treatments. Results of ANOVA tests ( $F$-values), Kruskal-Wallis $(\chi$-values) and $t$ tests for homogeneity of body length and colour score between focal and audience males are indicated. Kruskal-Wallis tests were used when residuals did not meet assumptions of normality.

\begin{tabular}{|c|c|c|c|c|c|c|c|}
\hline \multirow{2}{*}{ Treatment } & \multicolumn{3}{|c|}{ Male length (mm) } & \multicolumn{3}{|c|}{ Male colour score $(\%)$} & \multirow{2}{*}{$\begin{array}{l}\text { Stimulus female } \\
\text { length (mm) }\end{array}$} \\
\hline & Focal & Audience 1 & Audience 2 & Focal & Audience 1 & Audience 2 & \\
\hline No audience & $\begin{array}{c}19.28 \pm 0.28 \\
(16.88-22.51)\end{array}$ & - & - & $\begin{array}{c}4.01 \pm 0.37 \\
(0-7.57)\end{array}$ & - & - & $\begin{array}{c}26.61 \pm 0.65 \\
(18.44-31.95)\end{array}$ \\
\hline 1-male audience & $\begin{array}{c}19.44 \pm 0.31 \\
(16.74-21.89)\end{array}$ & $\begin{array}{c}19.46 \pm 0.3 \\
(15.81-22.28)\end{array}$ & - & $\begin{array}{c}3.99 \pm 0.38 \\
(0.74-8.83)\end{array}$ & $\begin{array}{c}3.72 \pm 0.44 \\
(0.34-9.64)\end{array}$ & - & $\begin{array}{c}27.67 \pm 0.52 \\
(22.25-32.75)\end{array}$ \\
\hline $\begin{array}{l}\text { Within treatment } \\
\text { comparisons }\end{array}$ & \multicolumn{3}{|c|}{$t_{50}=0.05, P=0.96$} & \multicolumn{3}{|c|}{$t_{50}=0.45, P=0.65$} & \\
\hline 2-male audience & $\begin{array}{c}19.71 \pm 0.29 \\
(17.87-22.56)\end{array}$ & $\begin{array}{c}19.06 \pm 0.3 \\
(17.08-21.57)\end{array}$ & $\begin{array}{c}19.41 \pm 0.33 \\
(17.13-22.78)\end{array}$ & $\begin{array}{c}4.5 \pm 0.56 \\
(1.24-12.63)\end{array}$ & $\begin{array}{c}3.55 \pm 0.35 \\
(0.56-5.92)\end{array}$ & $\begin{array}{c}3.42 \pm 0.33 \\
(0-9.18)\end{array}$ & $\begin{array}{c}27.89 \pm 0.58 \\
(23.55-34.82)\end{array}$ \\
\hline $\begin{array}{l}\text { Within treatment } \\
\text { comparisons }\end{array}$ & \multicolumn{3}{|c|}{$F_{2,63}=1.14, P=0.33$} & \multicolumn{3}{|c|}{$\chi_{2}=2.65, P=0.27$} & \\
\hline $\begin{array}{l}\text { Across treatment } \\
\text { comparisons }\end{array}$ & $\begin{array}{c}\mathrm{F}_{2,72}=0.52 \\
P=0.60\end{array}$ & \multicolumn{2}{|c|}{$F_{2,67}=0.47, P=0.63$} & $\begin{array}{l}\chi \chi=0.28 \\
P=0.87\end{array}$ & \multicolumn{2}{|c|}{$\chi_{2}=0.57, P=0.75$} & $\begin{array}{c}F_{2,72}=1.39, \\
P=0.26\end{array}$ \\
\hline
\end{tabular}




\subsection{Results}

Male guppies significantly reduced their overall mating effort in the presence of increasing numbers of rival audience males (non-parametric MANOVA, $F_{2,72}=2.317, P$ $=0.048$; Figure 8-2). This audience effect was reflected in similar monotonic decreases in the frequencies of courtship displays and sneak mating attempts and in the proportional use of courtship displays (relative to sneak mating attempts) across treatments, and was largely attributable to the significant difference in mating effort between the 0 and 2 audience male treatment levels (post-hoc MANOVA, $F_{1,47}=5.070, P=0.009$; Figure 82). In comparison, overall mating effort did not differ between the 0 vs. 1 audience male treatments $(P=0.275)$ nor between the 1 vs. 2 audience male treatments $(P=0.285)$.

Focal males and audience males did not differ in either their body length or colour ornamentation within and between treatments, and the body length of stimulus females did not vary across treatments (Table 8-1). Therefore, the observed differences in the mating behavioural tactics of focal males across the three audience treatment levels (Figure 8-2) cannot be explained by any variation in the body lengths and ornamentation of focal and stimulus males and in the body lengths of females. 

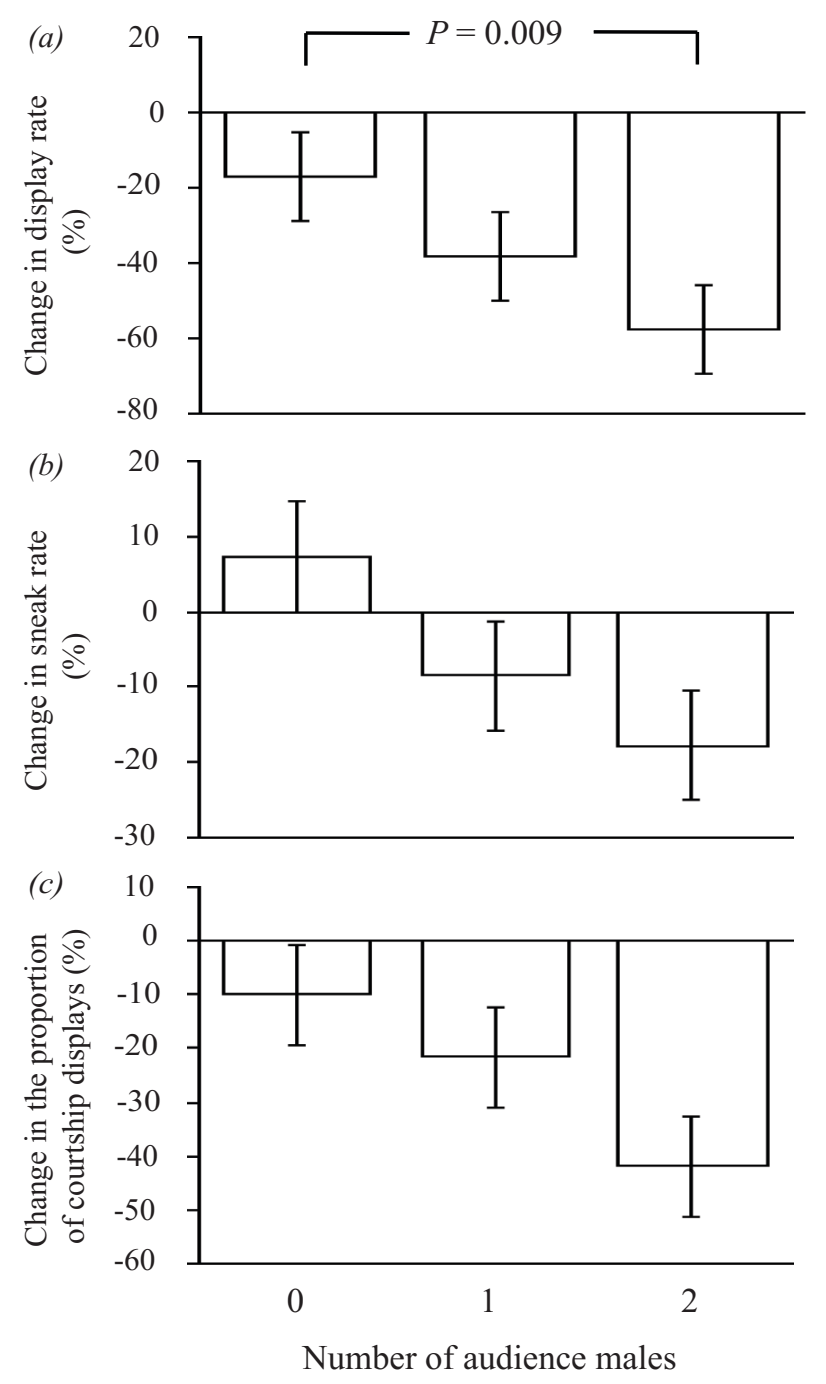

Figure 8-2 Comparison of the mating effort of focal males between two paired 20-min tests across three audience treatments: $(i)$ not being observed by a conspecific audience male ( 0 audience male present; Treatment 1$)$, or ( $i$ i) being observed by either an audience of one conspecific male (Treatment 2) or (iii) two conspecific males (Treatment 3). The mating behaviour of focal males is shown as the mean $( \pm \mathrm{SE})$ change in the frequencies of $(a)$ courtship displays and (b) sneak copulation attempts, and (c) the proportion of total mating attempts that are courtship displays between the paired tests. Only statistically significant paired comparisons across treatments (using permutational MANOVAs) are shown. 


\subsection{Discussion}

Males in some fish species (e.g. Dzieweczynski et al. 2005; Ziege et al. 2008, 2009), including the Trinidadian guppy (Auld and Godin 2015; Chapter 6), exhibit weaker mating preferences in the presence of a conspecific male audience compared to when an audience is not present. In species with alternative mating tactics, it remains unknown whether males also modify the relative use of their mating tactics in response to an audience of male sexual rivals nearby. Here, I report an audience effect on the mating effort of wild-caught male guppies from the Upper Aripo River. Males significantly reduced their overall mating effort, as measured by the rates of courtship displays and sneak mating attempts and the proportional use of courtship displays, with an increasing number of conspecific audience males present. The greatest effect was expressed in the presence of an audience of two sexual competitors. A reduction in costly courtship displays in particular was expected given that male Trinidadian guppies are sensitive to the ambient risk of sperm competition from sexual rivals and modify their mate choice behaviour in a manner than potentially minimizes this risk (Dosen and Montgomerie 2004b; Jeswiet et al. 2011b, 2012) and their alternative mating tactics are plastic in the face of varying ecological factors, such as predation (Godin 1995), ambient light levels (Chapman et al. 2009), and food availability and parasitism (Kolluru and Grether 2005; Kolluru et al. 2009).

The observed differences in the decrease in the frequency of courtship displays across my three experimental treatments suggests that focal males were aware of the nearby audience and adjusted their courting behaviour downwards most pronouncedly in the presence of an audience of two sexual rivals compared with the absence of an 
audience or the presence of only one sexual competitor. In doing so, focal males could have redirected the attention of the rival audience male(s) away from the female of interest, which could in turn potentially minimize their risk of sexual competition, consistent with the sexual competition avoidance hypothesis. Other studies on poeciliid fish have suggested that males strategically conceal their mating preferences from sexual (audience) rivals, or that they deceive rivals, by shifting their mating preference to another female nearby (Plath et al. 2008b; Bierbach et al. 2013), presumably to minimize sperm competition whilst pursuing a preferred female; a strategy that male guppies appear to exhibit as well (Chapters 6 - 7). Because of increased life-time fitness costs associated with increased sexual activity, including courtship (Miller and Brooks 2005; Jordan and Brooks 2010), selection should favour males who reduce overt courtship displays, and thereby overall energy expenditure, directed towards females who are not sexually receptive to their displays (Guevara-Fiore et al. 2010a) and thus unlikely to accept mating consensually (Houde 1997). In the current study, I purposely used wildcaught gravid females, who should therefore be unreceptive to male sexual advances, including courtship displays (Houde 1997), to minimize any confounding effect of female preference on male mating effort and to represent the most common reproductive state observed in wild female Trinidadian guppies, which is pregnancy and concurrent multiple paternity (Houde 1997; Kelly et al. 1999; Neff et al. 2008). I also controlled for variation in baseline mating effort between individual males by comparing a focal male's mating effort (courtship displays + sneak copulation attempts) towards a stimulus female whilst in the presence of an audience with his own mating effort towards that same female in the absence of an audience. However, so exposing individual focal males to sexually non- 
receptive females over a relatively lengthy trial period (i.e. over the course of paired Tests 1 and 2) could have partly contributed to the observed proportional decrease in male mating effort between the paired tests, but does not by itself explain the significant difference in the change in courtship display rate between Treatment 3 ( 2 audience males) and control Treatment 1 (no audience male).

My current results on male guppy courtship behaviour differ from those of a recent study by Nöbel and Witte (2013), who compared the courtship behaviour of male sailfin mollies in the presence or absence of a conspecific audience. They found that male mollies spent less time following a stimulus female, but did not alter the frequency of their courtship displays, when a male audience was present compared to when either no audience was present or when the audience was comprised of three sexually mature females. The latter study differed from ours in that the courtship behaviour of focal males whilst in the presence of an audience was not compared to their prior baseline courtship behaviour and the stimulus females used were sexually mature and receptive virgins. In a recent study on the guppy, Makowicz et al. (2010b) reported that, in the presence of a conspecific audience, focal males concurrently decreased the proportion of gonopodial thrusts (sneak mating attempts), but increased the proportion of courtship displays, directed towards the larger of two stimulus females presented, but neither change was statistically significant. Their results differ from ours, which could be owing to differences in fish provenance and experimental design between the two studies. Importantly, focal males in the Makowicz (2010b) study had simultaneous access to two stimulus females differing in body size (and potential fecundity), whereas focal males in my study had access to only one (sexually unreceptive) stimulus female to direct their 
mating effort towards. The strategic response of male guppies to the presence of an audience of sexual rivals may vary depending on the number of females available to them, as the latter would influence the benefits and costs of alternative male mating tactics.

Lastly, although male Upper Aripo guppies reduced the frequencies of their courtship displays and sneak mating attempts and their proportional use of courtship displays with an increase in the membership size of the audience (from one to two audience males), this increased response was not significant. Similarly, the magnitude of the decrease in the mating preferences of male guppies (from another Trinidadian population) observed in response to apparent competition from nearby sexual competitors did not vary significantly with an increase in the number (from one to two) of rival males present when paired stimulus females were matched in body length (Jeswiet et al. 2012). Competition from only one sexual rival may not be sufficient to select for male guppies to alter their mating behaviour so as to minimize their perceived risk of sexual competition, or an audience effect may not have been detectable in the presence of only one sexual rival due to uncontrolled variables, such as individual differences in prior mating experience (Jordan and Brooks 2012) and sperm production (Bretman et al. 2011b) among wild-caught males, in the current study.

Models of sperm competition (Parker et al. 1996) predict a biphasic male response to a perceived increase in their risk and intensity of sperm competition, such that males should increase their mating effort (and ejaculate investment) in the presence of one sexual competitor but decrease them in the presence of two or more sexual rivals. Contrary to sperm competition theory (Parker et al. 1996), male guppies in the current 
study showed a monotonic decrease in mating effort with increasing number (from 0 to 2) of potential sexual rivals nearby. Therefore, Upper Aripo male guppies were more likely adjusting their mating effort and alternative mating tactics in response to a perceived increased in pre-copulatory sexual competition (from the audience males) than to a perceived increase in their post-copulatory intensity of sperm competition in the current study. In the presence of rival males, a male faces increased pre-copulatory competition for physical access to females, as well as increased competition with respect to female choice (Andersson 1994; Houde 1997). A third potential alternative explanation for my results is that focal males (in the presence of other audience males nearby) could attract the attention of predators and therefore could be selected to reduce their mating effort, in particular their conspicuous courtship displays, when they perceive their risk of predation to have so increased (e.g. Godin 1995; Godin and Briggs 1996). However, this hypothesis does not explain my results because my source population (the Upper Aripo River) does not contain major fish predators and is thus considered a low predation population (Houde 1997; Magurran 2005).

In summary, I did obtain evidence for an audience effect on overall mating effort, in wild-caught male Trinidadian guppies. My results suggest that male guppies may minimize their risk of sexual competition in the presence of an audience of one or more rival males by reducing overall mating effort, as seen by a concordant decrease in the rates of both their courtship displays and sneak mating attempts and in the proportional use of courtship displays, rather than by only reducing their relative use of conspicuous courtship displays in favour of less conspicuous sneak mating attempts. However, I cannot rule out the possibility of an audience-mediated shift in the relative use of 
courtship displays and sneak copulation attempts in circumstances when males are courting sexually receptive females.

An animal's social environment, including an audience of sexual rivals, can influence their mating decisions and have important implications for sexual selection and thus for both direct and indirect genetic effects in a population (Moore et al. 1997). In this context, my finding of an audience effect on male mating effort is important. Building on my novel results with the Trinidadian guppy reported here, I encourage others to further investigate audience-mediated plasticity in alternative male mating tactics in the other species, a phenomenon that has surprisingly not been considered hitherto given that alternative mating strategies and tactics in animals has received considerable theoretical and empirical interest in the past (Gross 1996; Brockmann 2001).

\subsection{Acknowledgments}

I thank the Director of Fisheries, Ministry of Agriculture, Land and Marine Resources of Trinidad and Tobago for permission to collect fish from the Upper Aripo River, and G.E. Brown and C. Elvidge for assistance with fish collection, and I.W. Ramnarine for logistical support in Trinidad. 


\title{
Chapter 9:
}

Courtship Behaviour Attracts Male Sexual Rivals in the

\author{
Guppy
}




\subsection{Abstract}

Based on sexual and social selection theories, individual males are expected to socially associate with each other in ways that increase their chances of survival and(or) reproductive success. Because males can use available social information when making mating decisions, they should strategically associate with other males in a manner that provides them access to social information about potential mating opportunities.

Courtship displays directed by a male towards a female might provide inadvertent social information to nearby eavesdropping males about the perceived quality and(or) sexual receptivity of that female. Using the dichotomous choice paradigm, I tested whether eavesdropper male Trinidadian guppies (Poecilia reticulata) would preferentially associate with a demonstrator male (deemed the 'winner') who they had previously seen performing courtship displays towards a sexually-receptive female over another demonstrator male (deemed the 'loser') they had seen in close proximity to a female, but not performing any courtship displays. As predicted, following their observations of paired demonstrator males interacting with stimulus females, eavesdropping focal male guppies preferred to socially associate with the 'winner' demonstrator male over the paired 'loser' demonstrator male. However, when the winner male had visual access to the female, but did not perform courtship displays towards her, then focal males showed a strong social association preference for the loser male. These results suggest that it is the courtship displays per se of demonstrator males, and not simply their general behavioural interaction with females, that attracts other males as potential social partners. My findings highlight the importance of social behavioural cues and sexual signaling in the structuring of male-male social associations. 


\subsection{Introduction}

In social species, both males and females should socially associate non-randomly in a manner that increases their overall likelihood of survival and reproductive success. Studies have shown that multiple interacting variables, including genetic relatedness, physical characteristics (e.g., body size, sex) and(or) behavioural characteristics of potential social partners, can affect with whom individuals associate socially and the frequency and strength of those associations (e.g. Dugatkin and Sih 1995; Krause and Ruxton 2005; Croft et al. 2008). Important ecological drivers of sociality and social associations are those associated with survival benefits of group living, such as reduced individual risk of mortality from predators and enhanced foraging rates (Krause and Ruxton 2002). These fitness-related benefits can be further enhanced when associating with kin. Kin-based associations are common in many species of fishes and confer antipredator benefits (Ward and Hart 2003), physiological stress reduction and increased juvenile growth (Gerlach et al. 2007). Individuals also form social associations based on body size and coloration (e.g. Ward and Krause 2001; Krause and Ruxton 2002; Croft et al. 2005; Rodgers et al. 2011). Associating with individuals who are similar to oneself in either body size and(or) colour is hypothesized to reduce the 'oddity effect' and thus detection and attack by predators (Krause 1994; Krause and Ruxton 2002). There is also evidence that there are foraging benefits accrued by individuals when associating with phenotypically-similar conspecifics (Croft et al. 2009).

Sex and(or) reproductive status can also drive social associations between individuals (e.g. Snekser et al. 2006; Mourier et al. 2012). For example, in blacktip reef shark (Carcharhinus melanopterus) populations, individuals associate assortatively by 
both body length and sex (Mourier et al. 2012). In other species, there is evidence of females selectively associating with other females within their social network based on perceived sexual attractiveness or reproductive state. For example, female guppies (Poecilia reticulata) preferentially associate with other female guppies who are more sexually attractive to males, presumably to minimize their own sexual harassment from males (Brask et al. 2012). Female zebras assort by reproductive status (Sundaresan et al. 2007) and lactating female bats preferentially associate with other lactating females (Kerth and Konig 1999). There is also evidence of male-male associations being driven by mating opportunities or reproductive status. For example, bachelor zebras establish stronger social bonds with other bachelors than they do with stallions (Fischhoff et al. 2009). Male elephants preferentially associate with similarly-aged kin, which could benefit males through sparring play activities, which would later prepare them for malemale competition (Chiyo et al. 2011). Some male associations in dolphins are similarly thought to be driven by male-male competition (Lusseau 2007).

Based on sexual and social selection theories (Andersson 1994; Bateson and Healy 2005; McGlothin et al. 2010), males are expected to socially associate with each other in a manner which increases their individual chances of attracting females. To date, studies testing whether or not males associate with each other based on relative sexual attractiveness or sexual competitiveness in the guppy have used physical characteristics, such as body length and(or) colour ornamentation (Gasparini et al. 2013; Auge et al. 2016; S.J. Potter, H.L. Auld, T.N. Sherratt and J.-G.J. Godin, unpubl. data), as target traits. Together, the results of these studies suggest that in some populations male guppies will associate with other conspecific males based on their phenotypes, but in different 
ways depending on the circumstances (e.g. presence or absence of females). Using a population of feral guppies from Australia, Gasparini et al. (2013) found that males preferentially associated with males who were less sexually attractive than themselves when in the presence of females. In contrast, another study using wild-caught Trinidadian guppies from three different populations in Trinidad that vary in predation pressure found that, in the absence of females, males prefer to associate with males who are more attractive than themselves (Potter, Auld, Sherratt and Godin, unpubl. data). Moreover, a study using wild Upper Aripo River guppies (same as the current study’s guppy population) did not find any evidence for assortative or dissassortative male social partner choice based on either body length or colour ornamentation, in either the laboratory or the field (Auge et al. 2016).

In addition to physical traits, certain behavioural characteristics of males, such as propensity to perform courtship displays, can affect their sexual attractiveness to females (Andersson 1994). While females of numerous species use courtship displays to discriminate among males as potential mates (Andersson 1994), such displays are typically visually conspicuous and can reveal inadvertent social information (sensu Danchin et al. 2004) about the quality and(or) sexual receptivity of both the displaying males and the females being courted to male eavesdroppers and potential 'copiers' (see Chapter 5). When in the presence of an audience of either one or two sexual rivals, male guppies reduce their overall mating effort, as well as the proportion of their mating attempts that are courtship displays, compared to less conspicuous sneak mating attempts (see Chapter 8). By reducing their overall mating effort and the number of courtship displays, males minimize the overall amount of social information they inadvertently 
transmit through their sexual activities to eavesdropping male rivals, thereby minimizing their risk of their mate choice being later copied by eavesdroppers.

If male guppies are using social information to maximize their own reproductive success, then they should socially associate with other males who provide quality social information about their mating preferences and the quality of nearby females. If overt courtship displays are indicative of a male's sexual interest in a female and thus reveal information about the quality of that female as a mate, then males should seek to associate with other males who inadvertently provide social information about the quality of females by performing overt courtship displays. Additionally, males who perform courtship displays are likely to be sexually attractive to females (Andersson 1994; Houde 1997), which in turn could attract other males to their vicinity and thereby increase their encounter rate with sexually-receptive females than otherwise.

Here, using the dichotomous choice paradigm (e.g. Jeswiet and Godin 2011; Auge et al. 2016), I tested whether eavesdropping male Trinidadian guppies (Poecilia reticulata) would preferentially associate with a demonstrator male (deemed the 'winner') who they had previously seen performing courtship displays towards a sexually-receptive female over another demonstrator male (deemed the 'loser') they had seen in close proximity to a female, but not performing any courtship displays. I predicted that focal males would preferentially socially associate with the winner male, who he had previously observed courting a female. 


\subsection{Methods}

\subsubsection{Study animals}

I used laboratory-reared Trinidadian guppies as a study species. See Chapter 2 for a rationale.

\subsubsection{General protocol}

See Chapter 2 for a description of the common general protocol.

\subsubsection{Experimental apparatus}

For the first phase (viewing phase) of the experiment, the experimental apparatus consisted of a clear Plexiglas test aquarium (40 x 20 x $25 \mathrm{~cm}$; L x W x H; Figure 9-1) placed inside a warm water bath $(100 \times 100 \times 30 \mathrm{~cm}$; Figure 9-1). Water was $15 \mathrm{~cm}$ deep and maintained at a temperature of $24-26^{\circ} \mathrm{C}$. using two submersible Tetra ${ }^{\circledR} 100 \mathrm{~W}$ heaters. Five smaller, similar-sized and clear Plexiglas compartments (each 10 x 13 x $20 \mathrm{~cm}$ ) were placed adjacent to one another in a single row along one of the sides of the water bath and facing the larger viewing aquarium at a distance of $20 \mathrm{~cm}$ (Figure 9-1). A single narrow and perforated Plexiglas compartment (4 x 4 x $20 \mathrm{~cm}$; Figure 9-1) was placed inside the small compartment located at each end of the row. An opaque Plexiglas screen in front of each of the latter two end compartments obstructed the focal male's view into these compartments. A larger, removable opaque Plexiglas could be placed in front of the test aquarium, temporarily blocking the focal male's view of all the small compartments (Figure 9-1). The entire apparatus was placed behind a blind and illuminated overhead by three Phillips ${ }^{\circledR} 30 \mathrm{~W}$ fluorescent light tubes that were affixed above three sides of the 
water bath. The water bath, the viewing aquarium and all end compartments all contained a thin layer (approximate $1 \mathrm{~cm}$ ) of light-coloured gravel on the bottom.

In the second phase (social partner-choice phase) of the experiment, the experimental apparatus (Figure 9-2) consisted of a clear Plexiglas test aquarium $(40 \times 20 \times 25 \mathrm{~cm} ; \mathrm{L} \times \mathrm{W} \times \mathrm{H})$ flanked by a clear Plexiglas container $(6 \times 20 \times 25 \mathrm{~cm}$; hereafter termed compartment) at either end (see Chapter 2 for more details). The central aquarium served as the partner-choice arena and held the focal male, and the end compartments held the winner and loser demonstrator (stimulus) males. Removable opaque Plexiglas screens could temporarily block the focal male's view of both stimulus males. Since chemical cues can influence mate choice in animals (Candolin 2003), including the guppy (Shohet and Watt 2004), I prevented any exchange of chemical cues between the focal and stimulus males by placing the stimulus males in the end compartments outside of the central test aquarium, which also ensured that males could only use visual cues when assessing the stimulus males as potential social partners. See Chapter 2 for more information on general test aquaria conditions.

\subsubsection{Experimental protocol}

\subsubsection{Viewing phase}

First, a sexually-receptive, virgin stimulus female was haphazardly selected from a holding aquarium of virgin females and placed in the central stimulus compartment. Next, a focal male was selected from a separate all-male holding aquarium and placed in the larger viewing aquarium that faced the stimulus fish (Figure 9-1). From a different holding aquarium than the focal male, two other males that were matched in standard 
body length and ornamentation as closely as possible were then selected (see Chapter 2 for details on length and colour measurements, and Table 9-1) to serve as stimulus males. One of the paired stimulus males was designated as the 'winner' male and placed in one of the two compartments adjacent to the stimulus female (Figure 9-1); this male could view and court the stimulus female in the central compartment. The other paired stimulus male was designated as the 'loser' male and gently placed in the adjacent stimulus compartment on the other side of stimulus female, but could not view and court the stimulus female because of an opaque screen blocking his view into the central female compartment (Figure 9-1). The sides on which the winner and loser male were placed was alternated between trials. To control for any effect of potential differences in overall activity between the paired stimulus males, which could otherwise result from the winner male being able to see another fish (the stimulus female) while the loser male could not, a third male (a so-called 'dither' fish, Barlow 1968) was selected and placed into the far corner of the small compartment at the end of the row of compartments and adjacent to the loser male's compartment. This dither male was not visible to the focal male at anytime, nor to the stimulus female or winner male owing to opaque Plexiglas screens placed in front of the dither male's end compartment and between the loser male's compartment and the central female compartment (Figure 9-1). This set-up was counterbalanced by placing an empty dither male compartment inside the other far-end compartment adjacent to the winner male.

Once all fish were placed in their respective compartments, they were allowed to acclimatize for 5 min with opaque screens in place between all compartments and in front of the viewing aquarium, thus effectively preventing any visual contact between them. 
Following this acclimatization period, the opaque screens between the stimulus female and the winner male and between the loser male and the dither stimulus male were removed, and these stimulus fish were allowed to view each other for 5 min. During this period, the opaque screen between the focal male and the stimulus fish remained in place. After this brief viewing period for the stimulus fish, the focal male was given visual access to the stimulus fish and their behavioural interactions (through the clear walls of their respective compartments) by slowly removing the opaque screen in front of his viewing tank. The opaque screen between the stimulus female compartment and the compartment holding the loser male, as well as the opaque screen in front of the two dither male compartments at each end of the row of compartments, remained in place for the duration of the trial (Figure 9-1).

Immediately after the removal of the opaque screen in front of the focal male's viewing aquarium and once he resumed normal swimming behaviour, a 10-min viewing phase commenced. During this phase, the winner male had the opportunity to visually interact with the stimulus female and to perform courtships displays towards her. The loser male did not have visual access to the stimulus female and thus could not interact with her in any way or perform courtship displays towards her. However, the loser male did have visual access to a stimulus (dither) male in the far-end compartment adjacent to his compartment that none of the other fish could see. During this viewing phase, the amount of time that the focal male spent inside either of the viewing zones of his aquarium and directly facing either the distant winner or loser male compartment was recorded using stop watches. This viewing time was regarded as the time that the focal male spent visually 'assessing' the two stimulus males from a distance. Concurrently, the 
behaviour of the stimulus fish was recorded using a Canon VIXIA HF M500 digital video camera, positioned directly above the row of small compartments, for later analysis. Upon completion of the viewing phase, the focal male and the loser and winner stimulus males were gently dip-netted out of their respective aquarium/compartment and transferred to the partner-choice test aquarium (Figure 9-2). The stimulus female and the dither stimulus male were left in their respective compartments for later photography (see Chapter 2 for methodology).

\subsubsection{Social-partner choice phase}

Immediately following his removal from the viewing aquarium (Figure 9-1), the focal male was gently placed in a clear Plexiglas cylinder in the centre of the social partnerchoice test aquarium (Figure 9-2). The winner and loser stimulus males were then each introduced into one or the other of the two end compartments (Figure 9-2). The particular side (left or right) of the apparatus into which the winner and loser males were individually placed was the same side they were on during the viewing phase (Figure 91). See Chapter 2 for details on general protocol.

The fish were given a 5-min acclimatization period during which they did not have visual access with one other. After this period, I gently removed both opaque screens to allow visual contact between the focal male and both stimulus males. I then gently lifted the clear Plexiglas cylinder, allowing the focal male to swim freely in the test aquarium and choose to socialize with either stimulus males. During the next $10 \mathrm{~min}$, I observed the behaviour of the fish through a viewing window in the blind and recorded using stopwatches the amount of time that the focal male spent in the $10-\mathrm{cm}$ preference 
zone associated with each of the two stimulus males. At the end of this first 10-min period, I gently replaced the opaque screens (Figure 9-2) and gently dip-netted the focal male and returned him to the central clear Plexiglas cylinder in the middle of the test aquarium. To control for the possibility that the focal male might exhibit a preference (bias) for one side of the aquarium over the other rather than a preference for a particular stimulus male, I switched the sides of the end compartments holding the stimulus males (e.g. if the winner male was initially on the right-hand side of the test aquarium, then his end compartment was moved to the left-hand side of the aquarium, and vice-versa). Focal males exhibited a side bias (see Chapter 2 for details) in seven trials, which were discarded.

Once the the positions of the end compartments had been switched to control for any potential side bias of the focal male, I removed the opaque screens and lifted the Plexiglas cylinder to release the focal male and begin a second 10-min preference test (Preference test 2). This second preference test was identical to the first preference test (Preference test 1) described above. To calculate an overall preference score, I summed the amount of time that the focal male spent with the loser and winner male separately in Preference tests 1 and 2. The social preference score of the focal male for the winner stimulus male was expressed as the proportion of his total association time spent near $(<$ $10 \mathrm{~cm}$ ) that stimulus male (i.e. Sum of total association time spent near winner male / (Sum of total association time near the winner male + total association time near loser male)). At the end of second preference test, all fish were removed from the apparatus, lightly anesthetized and digitally photographed for later measurement of body length and quantification of male body ornamentation. See Chapter 2 for methodological details. 
After the social-partner choice phase was completed, I observed on a video monitor the behaviour of the fish during the 10-min viewing phase of the experiment using video playback. For each trial, I recorded the number of courtship displays exhibited by the winner male during this phase. Whilst observing the videos of stimulus fish behaviour, I realized that just less than half of all winner males courted the stimulus female. Because of this finding, two categories of stimulus 'winner' males were generated and regarded as separate treatments for the purpose of analysis, namely, winner males who courted the stimulus female $(N=15$, Table 9-1), and winner males who did not exhibit any courtship displays ( $N=18$, Table $9-1)$, despite visually interacting with the stimulus females, during the viewing period. Such a categorization of the winner stimulus males allowed me to discern whether focal males preferentially associated with winner males or not based on their previous observation of courtship displays exhibited or not by the winner males, and not simply because the latter males were seen interacting with a female.

To control for the possibility that males were associating with sexual rivals based on their courtship displays and interactions with females and not based simply on having been seen interacting with another fish, I carried a third treatment $(N=18$, Table 9-1) following the same protocol described above (viewing phase followed by social partnerchoice phase), except that in this control treatment the stimulus female in the central small compartment was replaced by a stimulus male to remove any possibility of courtship activity between either of the winner or loser males and the stimulus fish in the central compartment. Trials for the three treatments were carried out in random sequence. 


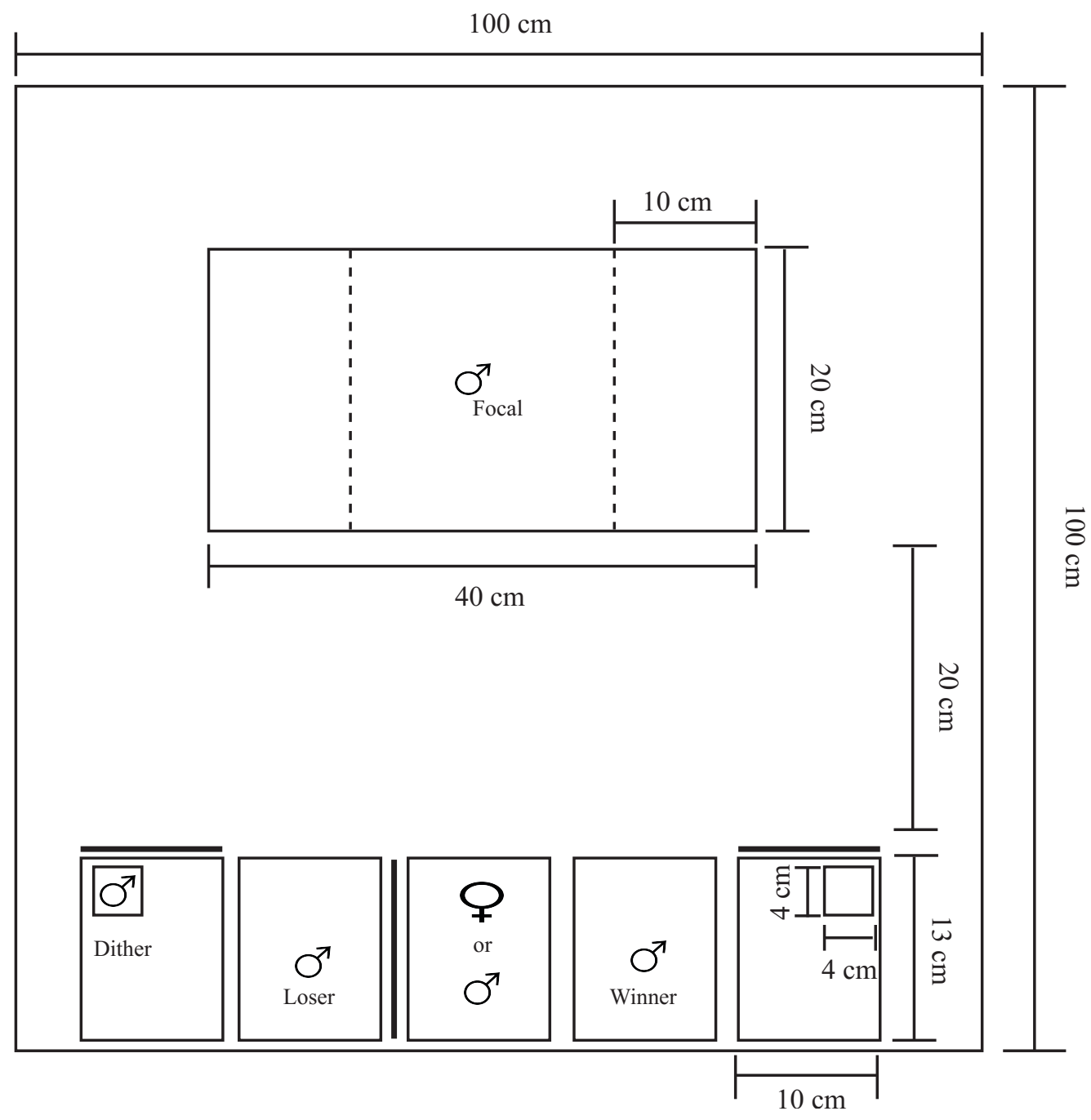

Figure 9 -1 Schematic top view of the experimental apparatus used in the viewing phase of the experiment. The larger viewing aquarium ( $40 \times 20 \times 25 \mathrm{~cm}$; L x W x H), which held the focal male, and the five smaller compartments $(6 \times 20 \times 25 \mathrm{~cm})$, which held the stimulus fish, were placed in a warm water bath $(100 \times 100 \times 30 \mathrm{~cm})$ as shown. The dashed lines represent $10-\mathrm{cm}$ viewing zones directly in line with the end compartments containing the stimulus males, and the thick solid lines represent removable opaque Plexiglas screens. The central small compartment held a stimulus female or male depending on the treatment (see Table 9-1). 


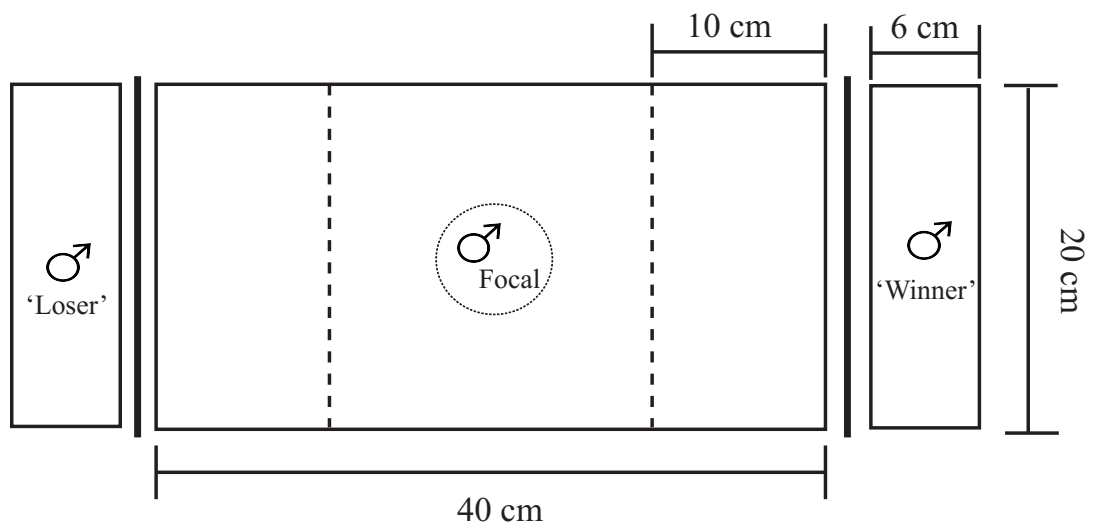

Figure 9-2 Schematic top view of the experimental apparatus used in the partner-choice phase of the experiment. The central compartment $(40 \times 20 \times 25 \mathrm{~cm}$; L x W x H) held the focal male and the end compartments $(6 \times 20 \times 22 \mathrm{~cm})$ held the 'winner' and 'loser' demonstrator stimulus males. The dashed lines represent 10-cm (social association) preference zones, and the thick solid lines represent removable opaque Plexiglas screens. The dotted circle denotes the removable clear Plexiglas cylinder $(7 \mathrm{~cm} \mathrm{diam}$.) that temporarily held the focal male. 
Table 9-1 Mean \pm SE (range) standard body length $(\mathrm{mm})$ and body ornamentation score (proportion of male body covered by orange and black pigmentation) of guppies used in the three treatments. Results of the ANOVA ( $F$-values), Kruskal-Wallis ( $\chi$-values), and $t$ tests for homogeneity of body length and colour score among male types, and results of $t$-tests comparing the body length of stimulus females between treatments, are shown. The Kruskal-Wallis test was used when residuals were not normally distributed.

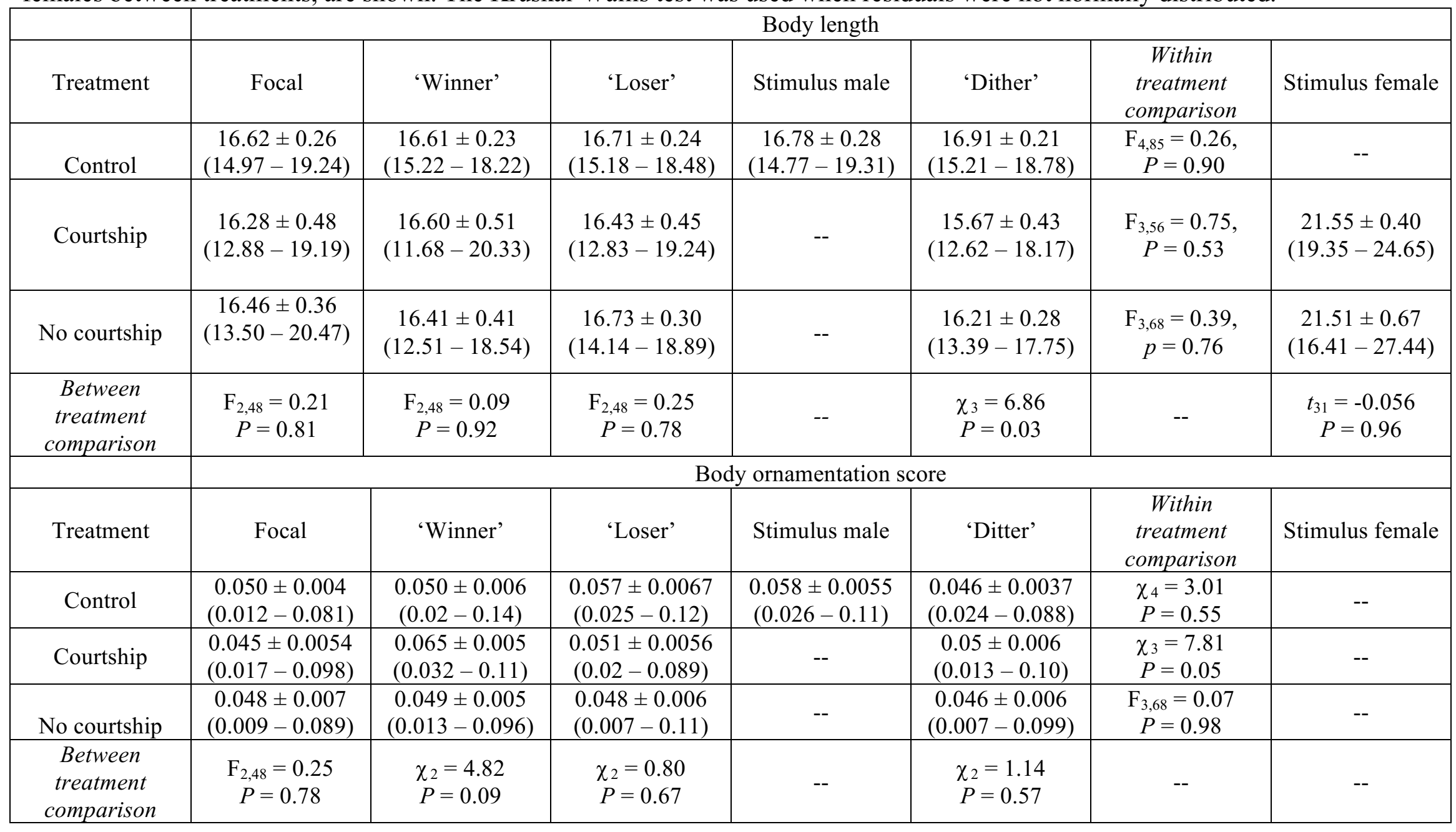




\subsubsection{Statistical analysis}

I first calculated the proportion of time that focal males spent in the viewing zone aligned in front of the winner male compartment (out of the total time spent in either viewing zone) during the viewing phase of the experiment, and then tested whether this proportion of viewing time in front of the winner male differed from that expected by chance (i,e. proportion $=0.50$ ) using a one-sample $t$-test for each of the three treatments separately.

To determine whether males make social partner-choice decisions based on the courtship rate of other males, I calculated the proportion of total social association time that focal males spent in the preference zone of the winner male for each treatment separately during the social partner-choice phase of the experiment. I then tested whether the proportion of total association time that the focal male spent in the preference zone associated with the winner male differed from that expected by chance (i.e. proportion $=$ $0.5)$ using the one-sample $t$-test. To compare the proportion of time that focal males spent associating with the winner stimulus male between the three treatments, I used oneway ANOVA, followed by a Tukey's test for post-hoc multiple comparison of means. I then used a linear model to compare the number of courtship displays performed by the winner stimulus male during the viewing phase and the proportion of time that the focal male subsequently spent socially associating with that stimulus male. Finally, I compared the proportion of time each focal male spent in the viewing zone in front of the winner male's compartment during the viewing phase and the proportion of time the focal male spent socially associating with the winner male in the social partner-choice test for each treatment separately using an ANOVA. 
When appropriate, proportional data were arcsine transformed. All $t$-tests used were two-tailed and all statistical analyses were performed using the R statistical framework (R Core Team 2014).

\subsubsection{Ethical standards}

This study was approved by the institutional Animal Care Committee at Carleton University (protocol \#13281) and thus meets the guidelines for the care and use of research animals of the Animal Behavior Society and the Canadian Council on Animal Care and the laws of Canada.

\subsection{Results}

There was no significant difference in the proportion of time that focal males spent in the viewing zone aligned in front of the winner stimulus male's compartment compared to that expected by chance when the latter male did not court the stimulus female (onesample $t$-test: $t_{17}=-0.22, P=0.83$; Figure 9-3a) or when the winner male courted the stimulus female (one-sample $t$-test: $t_{14}=0.90, P=0.38$; Figure 9-3a). However, focal males did spend significantly more time in the viewing zone aligned in front of the winner male's compartment in the control treatment, wherein the stimulus female was replaced by a stimulus male (one-sample $t$-test: $t_{17}=2.29, P=0.03$; Figure 9-3a).

After having observed the winner stimulus male court a stimulus female, focal males spent a greater, although marginally insignificant, proportion of their total association time in the preference zone of that male compared to that expected by chance (one-sample $t$-test: $t_{14}=1.98, P=0.07$; Figure 9-3b). In contrast, when the winner male 
did not court the stimulus female, focal males spent proportionally more time socially associating with the loser stimulus male than expected by chance (one-sample $t$-test: $t_{17}=$ $-4.07, P<0.001$; Figure 9-3b). Similarly, in the control treatment, focal males spent more time socially associating with the loser male than expected by chance (one-sample $t$-test: $t_{17}=-2.67, P=0.02$; Figure 9-3b). There was a significant difference in the proportion of social association time that focal males spent in the preference zone of the winner male between treatments (one-way ANOVA: $F_{2,51}=8.36, P<0.001$; Figure 9-3b). Post-hoc analysis revealed that focal males spent proportionately more time socially associating with the winner male when the latter had been previously observed to court a female during the viewing phase compared to when the winner male did not previously court the stimulus female $(P=0.008)$ or when a conspecific stimulus male was present in the central compartment rather than a virgin stimulus female $(P=0.008)$.

When the winner male courted the stimulus female during the viewing phase, the frequency of courtship displays performed by that male was positively (but marginally non-significant) related to the proportion of time the focal male subsequently spent social associating with the winner male during the social partner-choice phase (linear model: $t_{13}$ $\left.=2.13, R^{2}=0.26, P=0.053\right)$. Across all three treatments, the proportion of time that focal males spent in the viewing zone aligned with the compartment of the winner male during the viewing phase did not strongly predict the time they subsequently spent associating with the winner male during social partner-choice test (linear model: $t_{1,49}=$ $\left.2.07, R^{2}=0.06, P=0.04\right)$. 

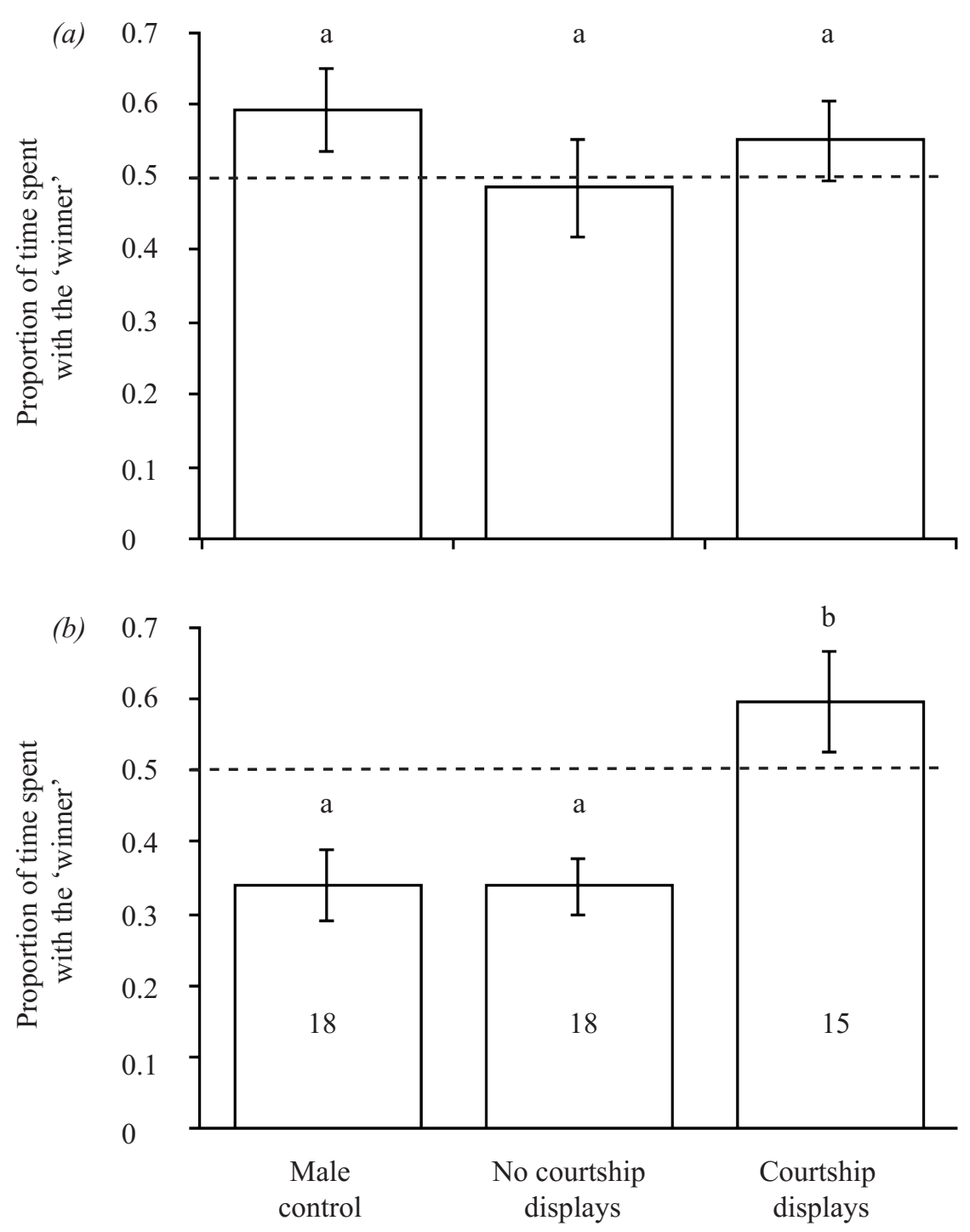

Figure 9-3 Mean \pm SE proportion of total association time the focal male spent near the winner stimulus male in the three treatments during $(a)$ the viewing phase and $(b)$ the social partner-choice phase of the experiment. The horizontal stippled lines at proportion 0.5 denote no choice (i.e. chance level). Histogram bars with dissimilar letters are significantly different $(P<0.05)$ from each other. In the male control treatment, the stimulus female in the central compartment of the viewing phase was replaced by a stimulus male. The number of replicate trials (with no side bias) for each treatment is shown inside inside the histogram bars. 


\subsection{Discussion}

In the current study, eavesdropping focal male guppies preferred to socially associate with a conspecific stimulus male (deemed the 'winner') who they had previously seen performing courtship displays towards a female over another nearby stimulus male (deemed the 'loser') who they had concurrently seen in proximity of the same stimulus female, but had not exhibited any courtship displays. There was a strong trend for a positive relationship between the courtship display rate of the winner stimulus males during the viewing phase and the strength of subsequent social association of the focal males for them. However, when the winner male did not perform any courtship displays towards the stimulus female or when the stimulus female was replaced with another stimulus male, focal males demonstrated a strong social association preference for the other (loser) stimulus male. These novel results suggest that male guppies who eavesdrop on the sexual interactions of other males are able to remember the identity of these males and to subsequently use social information gleaned from their past courtship behaviour, or lack thereof, to make decisions about which male conspecifics to socially associate with.

During the viewing phase of the experiment, focal males 'sampled' the aquarium zone in front of the winner and the loser male compartments equally when the stimulus fish in the central compartment was female. This is likely the result of the focal male being more interested in the stimulus female than either the adjacent winner or loser males and consequently spent most of his time in front of the stimulus female's central compartment during the viewing phase. When the stimulus fish in the central compartment was a male (control treatment), then the focal male spent significantly more 
time in front of the winner male's compartment than expected by chance, suggesting that the focal male was paying attention to the interaction between the winner male and the stimulus male.

Past studies (Dugatkin and Sargent 1994; Gasparini et al. 2013), which used different measures of male attractiveness, have found that male guppies prefer to associate with males who they perceive to be less attractive than themselves. Dugatkin and Sargent (1994) used male proximity to females as a proxy measure of their sexual attractiveness and found that eavesdropping males later showed a social association preference for the stimulus male that was observed further away from a female (i.e. 'loser') compared to another stimulus male that was similarly matched for body size and colour, but observed in closer proximity to a female. Stimulus males did not apparently court females in their study. Their result is consistent with my finding that males prefer to associate with the loser male in cases where neither the loser or winner stimulus males courted the stimulus female. While the compartments holding the paired winner and loser males were placed equidistant from the central stimulus female, the winner male had visual access to the female and thus spent more time interacting with her through the clear Plexiglas walls of their respective compartment, whereas the loser male did not. Because of this asymmetry, the eavesdropping focal male would have observed the winner stimulus male in close proximity (i.e. within three body lengths) of the stimulus female more often than the loser male.

When the winner stimulus male in this experiment performed courtship displays towards a sexually-receptive stimulus female, the eavesdropping focal male subsequently spent more time associating with the winner than the loser male, despite the winner male 
potentially appearing more sexually attractive to the female owing to his courtship displays directed towards her. The difference in the observed social association preferences of the focal male between the two treatments (i.e. winner male courting vs. not courting during the viewing phase) indicates that it is the courtship displays themselves that are socially attracting the focal male towards the winner stimulus male and not simply the general behavioural interactions between the winner male and stimulus female.

It is possible that the social attraction of eavesdropping focal male guppies towards the winner stimulus male, who they had previously seen courting a female, is the result of a sensory bias towards males actively performing courtship displays (Endler 1992). This proposition is supported by the finding of a positive, although marginally non-significant, relationship between the number of courtship displays performed by the winner male and the strength of the eavesdropping focal male's social association preference for the winner male. Furthermore, parasite-infected male guppies have lower courtship display rates than healthy males who are not infected by ectoparasites (Kolluru et al. 2009). Male guppies that are faced with a perceived increase in their ambient predation risk are more fearful and, in response, reduce their conspicuous courtship rate compared to males that are less threatened (Godin 1995). While it is unlikely that any males in the current experiment were parasitized since they were laboratory born and reared and showed no obvious sign of parasite infection, and there was no threat of predation during the experiment, eavesdropping focal males could have assessed the observed difference in courtship activity between the paired stimulus males as differential parasite load and(or) fear and subsequently avoided the male with the lower courtship 
rate and presumably associated higher parasite load and(or) fearfulness. The social attraction to the stimulus male who was observed displaying higher levels of activity may override any association preference for males who are perceived to be less sexually attractive as a result of differential proximity to a female. In the presence of sexual rivals, male guppies decrease their overall mating effort by decreasing both sneak mating attempts and courtship displays with a further decrease in the proportion of courtship displays relative to sneak mating attempts (Chapter 8). Additionally, a reduction in overall mating effort and a further decrease in courtship displays reduces the amount of social information available to eavesdropping rivals. Males may be more attracted to male social partners who continue to be active sexually and to thereby provide inadvertent social information than to other males who reduce or shut down their sexual behaviour and associated social information in the presence of sexual rivals.

Even though past studies using different measures of male sexual attractiveness and different populations have found that male guppies prefer to associate with less attractive males (Dugatkin and Sargent 1994; Gasparini et al. 2013), it is possible that eavesdropping focal male guppies in the current study preferred to socially associate with males who they had previously observed courting a female because they perceived these males as being more likely to attract other sexually-receptive females. By associating with such apparently attractive males, eavesdropping males might enhance their reproductive success through higher encounter rates with receptive females and(or) greater opportunities to sneak copulate females that are courted and attracted by their more attractive social partners. This result is consistent with the findings of a recent study (Potter, Auld, Sherratt and Godin, unpubl. data) that showed, in absence of females, male 
guppies from three different natural populations in Trinidad preferring to socially associated with rival males who were larger and more colour ornamented than themselves and thus more likely to be perceived as more sexually attractive and competitive. However, using the same study population (Upper Aripo River, Naranjo tributary) as I did in this experiment, Auge et al. (2016) found no male-male association preference based on either body size or colour ornamentation, both signals of sexual attractiveness and competitiveness in this population, in either the presence or absence of females. The difference in results between the latter study and my own suggest that either male guppies use behavioural cues over physical traits, such as body size and colour ornamentation, as signals of sexual attractiveness when assessing potential social partners or that males are increasing their association preference for males who they have previously seen courting females for other reasons, such as a sensory bias for males with increased activity levels, or an attraction to males who transmit social information regarding their perceived quality of nearby females.

The evolution of signals, such as courtship displays, in the guppy are affected by multiple factors including social structure, predation level, and ambient light (Endler 1992, 1995). In the current study, I used a single population of laboratory-reared, sexually inexperienced fish from a low-predation population and so my results may not be representative of how male guppies use behavioural cues when associating with social partners in other environments. For example, in high-predation environments, predation pressure could change the ways in which males use courtship displays when choosing social partners. 
The results from my study show that male guppies use behavioural cues from sexual rivals when making social partner-choice decisions. This highlights the importance of sexual behaviour in influencing male-male social associations, and thereby social networks, in nature. Future studies should investigate the mechanism underlying the observed preference of males to socially associate with males who they have previously observed courting females, while avoiding males who interact with females, but who do not court them.

\subsection{Acknowledgments}

I thank A.-C. Auge, D. Choudhury, R. Pusiak, V. Scarponi, G. Mazué, and S. Potter for assistance with fish care, and R. Pusiak for assistance with the analysis of fish photographs. 


\section{Chapter 10:}

General Thesis Conclusions 


\subsection{Salient findings and conclusions}

In this thesis, I have shown that male Trinidadian guppies, like their female counterparts, from the Upper Aripo River population in Trinidad have repeatable and consistent mating preferences in the absence of available social information (Chapter 4), but when social information is available (e.g. through observing the behavioural interactions of others), males can flexibly adjust their mating behaviour. Similar to males in many animal species, male guppies from my study population exhibited an overall preference for larger, and presumably more fecund, females in all experiments that measured male mate choice in the absence of available social information (Chapters 4 - 7). Even though males generally had a preference for larger females, there was nonetheless considerable variation among individuals in the strength and direction of their mating preferences (Chapter 4). Expression of male mating preferences could change over the course of a male's lifetime and is likely to be constrained by female preference to some degree.

As expected, female guppies in my study population demonstrated strong mating preferences for larger males over smaller ones, but a surprisingly weaker (but nonetheless significant) preference for colour ornamentation given that they originated from a population with low predation pressure (Chapter 3). Larger, and presumably more desirable females, showed stronger mating preferences for larger males than did smaller females, suggesting at least some degree of assortative mating. These results thus contribute further to our understanding of both intra- and inter-population variation in mating preferences in the Trinidadian guppy, a long-standing important model species for the study of sexual selection and evolution, and should encourage further investigations 
into size-assortative mating and potential interactions between male body length and ornamentation on female mate choice in this model species and others.

When provided with the opportunity to supplement their own privately acquired information regarding the quality of nearby potential mates, males copied the mate choice of rival males who they had observed near an initially non-preferred female (Chapter 5). Copying the mate choice of another male could reduce the costs and associated with searching for a high-quality and sexually-receptive mate. In species with some degree of last male sperm precedence, such as the guppy, copying the mate choice of another male would result in asymmetrical levels of sperm competition with the first male to mate suffering a greater cost (lost of paternity) than the 'copier'.

As would be predicted if males were responding to an increased risk of sexual competition in the presence of an audience male, males decreased their overall mating effort by decreasing their total number of courtship displays and total number of sneak mating attempts. Furthermore, they decreased their relative use of conspicuous courtship displays compared to less conspicuous sneaky mating attempts in the presence of either one or two nearby sexual rivals (Chapter 8). Males also decreased their preference for an initially-preferred female and consequently increased their preference for an initially nonpreferred female in the presence of one or two sexual rivals (Chapter 6). Whether or not a male changed his mate preference in the presence of an audience was contingent on the strength of his preference for his initially-preferred female and the phenotype of the audience male (Chapter 7). Males who initially had a very strong preference for one female over the other exhibited a greater change in their preference irrespective of whether or not the initially-preferred female was the larger, and presumably more 
desirable, of the two females (Chapter 7). Males decreased their initial mating preference for a particular female when the audience male was larger than himself, but not when the audience male was smaller than himself. This increased reduction in preference for an initially preferred female in the presence of a larger rival audience male is consistent with males avoiding sexual competition in the presence of a more competitive sexual rival. Larger males are likely to be better sexual competitors as females prefer larger males as mates (Chapter 3), they produce more sperm, and they are likely to be able to physically outcompete smaller males for access to females. Altogether, these results demonstrate plasticity in male mating behaviour, including mate choice, and highlight the importance of social information and the social environment in determining male mating behaviour.

Because a male's social environment and by extension his social partners can affect his mating behaviour and thus reproductive success, males should associate with other males in a way which enhances their individual reproductive success. As would be expected if males were associating with other males to increase their own reproductive success, male guppies preferred to associate with conspecific males who they had previously seen courting sexually-receptive virgin females, suggesting that males will preferentially associate with other males based upon the latter's sexual attractiveness and(or) their propensity to provide social information whilst interacting with females.

Altogether, the experimental results from my thesis importantly demonstrate that although male guppies have repeatable and inherent mating preferences, they are also highly sensitive to their social environment and are able to use public information to flexibly adjust their mating preferences, mating effort and alternative mating tactics in ways that appear to be adaptive. These socially-mediated changes in the mating 
behaviour of males modify the expression of inherent mating preferences and potentially plays an important role in influencing the direction and strength of sexual selection and maintenance of variation in populations, which is critical for the operation of selection and further adaptive evolution.

\subsection{Future studies}

The results from my thesis research generated more questions about how males use social information when making mate-choice decisions, how they respond to the presence of an audience of sexual rivals, and how their socially-mediated mating decisions might affect male-male associations (i.e. social partner choices) in nature.

In Chapter 5 of my thesis, I demonstrated male mate choice copying in the guppy when the focal male (copier) and model male (demonstrator) were similarly matched for body length and colour ornamentation. However, males may use available social information discriminately. For example, when female guppies copy the mate choice of other females, they do so selectively by only copying the mate choice of older, more experienced females who would presumably be able to transmit higher quality information (Dugatkin and Godin 1993; Vukomanovic and Rodd 2007). Similar to their female counterparts, male guppies should selectively copy the mate choice of other males in order to maximize their reproductive success. Future research should include behavioural studies that test whether or not males are selective when copying another male's mate choice. Similarly, future research should test whether certain types of males, for example younger ones, are more likely to use available social information when making mating decisions. For example, there is some evidence that personality type 
affects an individual's propensity to use social information (e.g. Kurvers et al. 2010) and how they respond to an audience of sexual rivals (Bierbach et al. 2015), which in turn may influence his/her likelihood of copying the mate choice of other individuals. In Chapters 6 and 8, I demonstrated that male guppies will respond to the presence of an audience of sexual rivals, and thus to the risk of being copied and the subsequent increase in sexual competition, by altering their apparent mate choice or decreasing their sexual activity. More work is needed to understand how male mate choice copying and the audience effect co-exist in a population, as the existence of the audience effect should decrease the likelihood of male mate choice copying. In Chapter 7, I showed that males will respond the presence of an audience by modifying their inherent mating preference to a greater extent in the presence of larger males, but not more ornamented, audience males. Future work should seek to determine why males respond differently to the presence of larger males compared to more colour ornamented males, given that both are likely to be perceived as strong sexual competitors in my study population. Building on my novel results on mate choice copying and the audience effect with the Trinidadian guppy reported here (Chapters 5 - 8), I encourage others to further investigate audiencemediated plasticity in alternative male mating tactics in other species, a phenomenon that has surprisingly not been considered before now given that alternative mating strategies and tactics in animals has received considerable theoretical and empirical interest in the past (Gross 1996; Brockmann 2001).

In Chapter 9, I showed that eavesdropping male guppies prefer to socially associate with male conspecifics who they have recently seen courting a sexuallyreceptive female over other males that were observed interacting with, but not courting, a 
female. However, my experiment was unable to tease apart whether males were attracted to males who performed courtship displays because those males would be viewed as more sexually attractive to females, because they provide social information regarding the quality of nearby females, or simply because they demonstrate higher overall activity levels. Future studies should address the question of why male guppies prefer to socially associate with other males who they have seen perform courtship displays towards females.

The fish used in my experimental studies originated from a natural low-predation population (Upper Aripo River) in Trinidad. Predation level is known to influence mate choice in the guppy (Godin and Briggs 1996) and in other species (e.g. Teyssier et al. 2014) and could similarly influence how males use social information when making mating decisions. It would be interesting to study the effects of predation risk on whether and how males use social information when making decisions, particularly as they relate to mating effort and mate choice because of their central importance to sexual selection.

Lastly, future research into the effects of social information on male mate choice should incorporate genetic work to test whether males gain any fitness benefits, such as an increase in offspring number and(or) quality, when copying the mate choice of other males or flexibly adjusting their mating preference, mating effort or mating tactics in the presence of an audience of sexual rivals. 


\section{References Cited}

Abrahams, M.V. 1993. The trade-off between foraging and courting in male guppies. Animal Behaviour, 45: 673-681.

Alonzo, S. H. (2008). Female mate choice copying affects sexual selection in wild populations of the ocellated wrasse. Animal Behaviour, 75(5), 1715-1723.

Amundsen, T. 2000. Why are female birds ornamented? Trends in Ecology and Evolution, 15: 149-155.

Andersson, M.B. 1994. Sexual Selection. Princeton University Press, Princeton.

Andersson, M.B. and Simmons, L.W. 2006. Sexual selection and mate choice. Trends in Ecology and Evolution, 21: 296-302.

Anderson, M.J. 2001. A new method for non-parametric multivariate analysis of variance. Austral Ecology, 26:32-46.

Auge, A.-C., Auld, H.L., Sherratt, T.N. and Godin, J.-G.J. 2016. Do males form social associations based on sexual attractiveness in a fission-fussion fish society? PLoS ONE, 11(3): e0151243. 
Auld, H.L. and Godin, J.-G.J. 2015. Sexual voyeurs and copiers: social copying and the audience effect on male mate choice in the guppy. Behavioral Ecology and Sociobiology, 69: $1795-1807$.

Auld, H.L., Jeswiet, S.B. and Godin, J.-G.J. 2015. Do male Trinidadian guppies adjust their alternative mating tactics in the presence of a rival male audience? Behavioral Ecology and Sociobiology, 69: 1191-1199.

Auld, H.L., Punzalan, D., Godin, J.-G.J. and Rundle, H.D. 2009. Do female fruit flies (Drosophila serrata) copy the mate choice of others? Behavioral Processes, 82: 78-80.

Auld, H.L., Pusiak, R.J.P. and Godin, J.-G.J. 2016. Independent mating preferences for male body size and coloration in female Trinidadian guppies. Ethology, 122: 597-608.

Bakker, T.C.M. and Pomiankowski, A. 1995. The genetic basis of female mate preferences. Journal of Evolutionary Biology, 8: 129-171.

Baldauf, S.A., Kullmann, H., Schroth, S.H., Thünken, T. and Bakker, T.C. 2009. You can't always get what you want: size assortative mating by mutual mate choice as a resolution of sexual conflict. BMC Evolutionary Biology, 9: 129.

Barlow, G.W. 1968. Dither - a way to reduce undesirable fright behavior in ethological studies. Zeitschrift für Tierpsychologie. 25: 315-318. 
Bateson, M. and Healy, S.D. 2005. Comparative evaluation and its implications for mate choice. Trends in Ecology and Evolution. 20: 659-664.

Bell, A.M., Hankinson, S.J. and Laskowski, K.L. 2009. The repeatability of behaviour: a meta-analysis. Animal Behaviour, 77: 771-783.

Bel-Venner, M.C., Dray, S., Allaine, D., Menu, F. and Venner, S. 2008. Unexpected male choosiness for mates in a spider. Proceedings of the Royal Society B, 275: 77-82.

Benjamini, Y. and Hochberg, Y. 1995. Controlling the false discovery rate: a practical and powerful approach to multiple testing. Journal of the Royal Statistical Society B, 57: 289-300.

Bierbach, D., Girndt, A., Hamfler, S., Klein, M., Müksch, F., Penshorn, M., Schwinn, M., Zimmer, C., Schlupp, I., Streit, B., Plath, M. 2011a. Male fish use prior knowledge about rivals to adjust their mate choice. Biology Letters, 7: 349-351.

Bierbach, D., Kronmarck, C., Hennige-Schulz, C., Stadler, S. and Plath, M. 2011 b. Sperm competition risk affects male mate choice copying. Behavioral Ecology and Sociobiology, 65: 1699-1707. 
Bierbach, D., Madlen, Z., Zimmer, C., Riesch, R., Schlupp, I. and Plath, M. 2012. Male Grijalva mosquitofish (Heterophallus milleri) increase mating preferences in front of an audience. Aqua, 18: 198-208.

Bierbach, D., Makowicz, A.M., Schlupp, I., Geupel, H., Streit, B. and Plath, M. 2013. Casanovas are liars: behavioral syndromes, sperm competition risk, and the evolution of deceptive male mating behavior in live-bearing fishes. F1000 Research 2013, 2: 75.

Bierbach, D., Sommer-Trembo, C., Hanisch, J., Wolf, M. and Plath, M. 2015. Personality affects mate choice: bolder males show stronger audience effects under high competition. Behavioural Ecology, 26: 1314-1325.

Bisazza, A., Marconato, A. and Marin, G. 1989. Male mate preferences in the mosquitofish Gambusia holbrooki. Ethology, 83: 335-343.

Bischoff, R.J., Gould, J.L. and Rubenstein, D.I. 1985. Tail size and female choice in the guppy (Poecilia reticulata). Behavioral Ecology and Sociobiology, 17: 253-255.

Boake, C.R.B. 1989. Repeatability: its role in evolutionary studies of mating behavior. Evolutionary Ecology, 3: 173-182.

Bonduriansky, R. 2001. The evolution of male mate choice in insects: a synthesis of ideas and evidence. Biology Reviews, 76: 305-339. 
Bradbury, J.W. and Vehrencamp, S.L. 1998. Signals and communication. In: Principles of Animal Communication, Second edition. Sinauer Associates, Sunderland, MA.

Brask, J.B., Croft, D.P., Thompson, K., Dabelsteen, T. and Darden, S.K. 2012. Social preferences based on sexual attractiveness: a female strategy to reduce male sexual attention. Proceedings of the Royal Society B, 279: 1748-1753.

Breden, F. and Stoner, G. 1987. Male predation risk determines female preference in the Trinidad guppy. Nature, 329: 831-833.

Bretman, A., Gage, M.J.G. and Chapman, T. 2011a. Quick-change artists: male plastic behavioural responses to rivals. Trends in Ecology and Evolution, 26: 467-473.

Bretman, A., Westmancoat, J.D., Gage, M.J.G. and Chapman, T. 2011b. Males use multiple, redundant cues to detect mating rivals. Current Biology, 21: 617-622.

Briggs, S.E., Godin, J.-G.J. and Dugatkin, L.A. 1996. Mate-choice copying under predation risk in the Trinidadian guppy (Poecilia reticulata). Behavioral Ecology, 7: $151-157$.

Brockmann, H.J. 2001. The evolution of alternative strategies and tactics. Advances in the Study of Behavior, 30: 1-51. 
Brooks, R. 2002. Variation in female mate choice within guppy populations: population divergence, multiple ornaments and the maintenance of polymorphism. Genetica, 116: $343-358$.

Brooks, R. and Endler, J.A. 2001. Female guppies agree to differ: phenotypic and genetic variation in mate-choice behavior and the consequences for sexual selection. Evolution, 55: $1644-1655$.

Brown, G.R. and Fawcett, T.W. 2005. Sexual selection: copycat mating in birds. Current Biology, 15: R626-R628.

Brown, W.D. 1990. Size-assortative mating in the blister beetle Lytta magister (Coleoptera: Meloidae) is due to male and female preference for larger mates. Animal Behaviour, 40: 901-909.

Burns, J.G. and Rodd, F.H. 2008. Hastiness, brain size and predation regime affect the performance of wild guppies in a spatial memory task. Animal Behaviour, 76: 911-922.

Callander, S., Hayes, C.L., Jennions, M.D. and Backwell, P.R.Y. 2013. Experimental evidence that immediate neighbors affect male attractiveness. Behavioral Ecology, 24: $730-733$. 
Candolin, U. 2003. The use of multiple cues in mate choice. Biological Reviews, 78: 575595.

Castellano, S., Friard, O. and Pilastro, A. 2016. The audience effect and the role of deception in the expression of male mating preferences. Animal Behaviour, 115: 273282.

Chapman, B.B., Morrell, L.J. and Krause, J. 2009. Plasticity in male courtship behaviour as a function of light intensity in guppies. Behavioral Ecology Sociobiology, 63: 17571763.

Chenoweth, S.F. and Blows, M.W. 2006. Dissecting the complex genetic basis of mate choice. Nature Reviews Genetics, 7: 681-692.

Chiyo, P.I., Archie, E.A., Hollister-Smith, J.A., Lee, P.C., Poole, J.H., Moss, C.J. and Alberts, S.C. 2011. Association patterns of African elephants in all-male groups: the role of age and genetic relatedness. Animal Behaviour, 81: 1093-1099.

Clutton-Brock, T.H. 2007. Sexual selection in males and females. Science, 318: 18821885.

Clutton-Brock, T.H. and Vincent, A.C.J. 1991. Sexual selection and the potential reproductive rates of males and females. Nature, 351: 58-60. 
Cotton, S., Small, J. and Pomiankowski, A. 2006. Sexual selection and conditiondependent mate preferences. Current Biology, 16: R755-R765.

Crawley, M.J. 2007. The R Book. John Wiley and Sons Ltd, Chichester, UK.

Croft, D.P., Arrowsmith, B.J., Bielby, J., Skinner, K., White, E., Couzin, I.D., Magurran, A.E., Ramnarine, I. and Krause, J. 2003. Mechanisms underlying shoal composition in the Trinidadian guppy, Poecilia reticulata. Oikos, 100: 429-438.

Croft, D.P., James, R. and Krause, J. 2008. Exploring Animal Social Networks. Princeton University Press, Princeton.

Croft, D.P., James, R., Ward, A.J.W., Botham, M.S., Mawdsley, D. and Krause, J. 2005. Assortative interactions and social networks in fish. Oecologia, 143: 211-219.

Croft, D.P., Krause, J., Darden, S.K., Ramnarine, I.W., Faria, J.J. and James, R. 2009. Behavioural trait assortment in a social network: patterns and implications. Behavioral Ecology and Sociobiology, 63: 1495-1503.

Croft, D.P., Morrell, L.J., Wade, A.S., Piyapong, C., Ioannou, C.C., Dyer, J.R.G., Chapman, B.B., Wong, Y. and Krause, J. 2006. Predation risk as a driving force for sexual segregation: a cross-population comparison. American Naturalist, 167: 867-878. 
Cummings, M.E. 2015. The mate choice mind: studying mate preference, aversion and social cognition in the female poeciliid brain. Animal Behaviour, 103: 249-258.

Dabelsteen, T. 2005. Public, private or anonymous? Facilitating and countering eavesdropping. In: McGregor, P.K. (ed), Animal Communication Networks. Cambridge University Press, Cambridge, pp 38-62.

Dall, S. R., Giraldeau, L.A., Olsson, O., McNamara, J.M. and Stephens, D.W. 2005. Information and its use by animals in evolutionary ecology. Trends in ecology \& evolution, 20: 187-193.

Danchin, E., Giraldeau, L.-A., Valone, T.J. and Wagner, R.H. 2004. Public information: from nosy neighbors to cultural evolution. Science, 305: 487-491.

Del Re, A.C. 2013. compute.es: Compute Effect Sizes. R package version 0.2-2. URL http://cran.r-project.org/web/packages/compute.es

Dohm, M.R. 2002. Repeatability measures do not always set an upper limit to heritability. Functional Biology, 16: 273-280.

Dosen, L.D. and Montgomerie, R. 2004a. Female size influences mate preferences of male guppies. Ethology, 110: 245-255. 
Dosen, L.D. and Montgomerie, R. 2004b. Mate preferences by male guppies (Poecilia reticulata) in relation to the risk of sperm competition. Behavioral Ecology and Sociobiology, 55: 266-271.

Dougherty, L.R. and Shuker, D.M. 2015. The effect of experimental design on the measurement of mate choice: a meta-analysis. Behavioral Ecology, 26: 311-319.

Dubois, F. 2015. When being the centre of the attention is detrimental: copier may favour the use of evasive tactics. Behavioral Ecology and Sociobiology, 69: 183-191.

Dubois, F. and Belzile, A. 2013. Audience effect alters male mating preferences in zebra finches (Taeniopygia guttata). PLoS ONE, 7: e43697.

Dugatkin, L.A. 1992. Sexual selection and imitation: Females copy the mate choice of others. American Naturalist, 199: 1384-1389.

Dugatkin, L.A. and Godin, J.-G.J. 1992. Reversal of female mate choice by copying in the guppy (Poecilia reticulata). Proceedings of the Royal Society B, 249: 179-184.

Dugatkin, L.A. and Godin, J.-G.J. 1993. Female mate copying in the guppy (Poecilia reticulata): age-dependent effects. Behavioral Ecology and Sociobiology, 4: 289-292. 
Dugatkin, L.A., Lucas, J.S. and Godin, J.-G.J. 2002. Serial effects of mate-choice copying in the guppy (Poecilia reticulata). Ethology Ecology and Evolution, 14: 45-52.

Dugatkin, L.A. and Sargent R.C. 1994. Male-male association patterns and female proximity in the guppy, Poecilia reticulata. Behavioral Ecology and Sociobiology, 35: 141-145.

Dugatkin, L.A. and Sih, A. 1995. Behavioral ecology and the study of partner choice. Ethology. 99: 265-277.

Dzieweczynski, T.L., Earley, R.L., Green, T.M. and Rowland, W.J. 2005. Audience effect is context dependent in Siamese fighting fish, Betta splendens. Behavioral Ecology and Sociobiology, 16: 1025-1030.

Dzieweczynski, T.L. and Walsh, M.M. 2010. Audience type and receptivity affect malefemale interactions in Siamese fighting fish. Ethology, 117: 10-18.

Edward, D.A. and Chapman, T. 2011. The evolution and significance of male mate choice. Trends in Ecology and Evolution, 26: 647-654.

Edward, D.A. and Chapman, T. 2012. Measuring the fitness benefits of male mate choice in Drosophila melanogaster. Evolution, 66: 2646-2653. 
Endler, J.A. 1980. Natural selection on color patterns in Poecilia reticulata. Evolution, 34: 76-91.

Endler, J.A. 1987. Predation, light intensity and courtship behaviour in Poecilia reticulata (Pisces, Poeciliidae). Animal Behaviour, 35: 1376-1385.

Endler, J.A. 1991. Variation in the appearance of guppy color patterns to guppies and their predators under different visual conditions. Vision Research, 31: 587-5608.

Endler, J.A. 1992. Signals, signal conditions, and the direction of evolution. American Naturalist, S125-S153.

Endler, J.A. 1995. Multiple-trait coevolution and environmental gradients in guppies. Trends in Ecology and Evolution, 10: 22-29.

Endler, J.A. and Houde, A.E. 1995. Geographic variation in female preferences for male traits in Poecilia reticulata. Evolution, 49: 456-468.

Evans, C. and Marler, P. 1994. Food calling and audience effects in male chickens, Gallus gallus: Their relationships to food availability, courtship and social facilitation. Animal Behaviour, 47: 1159-1170. 
Evans, J.P., Bisazza, A. and Pilastro, A. 2004. Female mating preferences for colourful males in a population of guppies subject to high predation. Journal of Fish Biology, 65: 1154-1159.

Evans, J.P. and Magurran, A.E. 2001. Patterns of sperm precedence and predictors of paternity in the Trinidadian guppy. Proceedings of the Royal Society B, 268: 719-724.

Evans, J.P., Pilastro, A. and Ramnarine, I.W. 2003a. Sperm transfer through forced matings and its evolutionary implications in natural guppy (Poecilia reticulata) populations. Biological Journal of the Linnean Society, 78: 605-612.

Evans, J.P., Zane, L., Francescato, S. and Pilastro, A. 2003b. Directional postcopulatory sexual selection revealed by artificial insemination. Nature, 421: 360-363.

Field, A., Miles, J. and Field, Z. 2012. Discovering Statistics Using R. Sage Publications Ltd., London, UK.

Fischhoff, I.R., Dushoff, J., Sundaresan, S.R., Cordingley, J.E. and Rubenstein, D.I. 2009. Reproductive status influences group size and persistence of bonds in male plains zebra (Equus burchelli). Behavioral Ecology and Sociobiology, 63: 1035-1043.

Fisher, H.S. and Rosenthal, G.G. 2007. Male swordtails court with an audience in mind. Biology Letters, 3: 5-7. 
Fisher, R.A. 1930. The Genetical Theory of Natural Selection. Oxford University Press, Oxford.

Frommen, J.G., Rahn, A.K., Schroth, S.H., Waltschyk, N. and Bakker, T.C.M. 2009. Mate-choice copying when both sexes face high costs of reproduction. Evolutionary Ecology, 23: 435-446.

Galef, B.G. and White, D.J. 1998. Mate-choice copying in Japanese quail, Coturnix coturnix japonica. Animal Behaviour, 55: 545-552.

Gasparini, C., Serena, G. and Pilastro, A. 2013. Do unattractive friends make you look better? Context-dependent male mating preferences in the guppy. Proceedings of the Royal Society B, 280: 20123072.

Gerlach, G., Hodgins-Davis, A., MacDonald, B. and Hannah, R.C. 2007. Benefits of kin association: related and familiar zebrafish larvae (Danio rerio) show improved growth. Behavioral Ecology and Sociobiology, 61: 1765-1770.

Ghalambor, C.K., Reznick, D.N. and Walker, J.A. 2004. Constraints on adaptive evolution: the functional trade-off between reproduction and fast-start swimming performance in the Trinidadian guppy (Poecilia reticulata). American Naturalist, 164: $38-50$. 
Gilburn, A.S., Foster, S.P. and Day, T.H. 1992. Female mating preference for large size in Coelopa frigida (seaweed fly). Heredity, 69: 209-216.

Godin, J.-G.J. 1995. Predation risk and alternative mating tactics in male Trinidadian guppies (Poecilia reticulata). Oecologia, 103: 224-229.

Godin, J.-G.J. and Auld, H.L. 2013. Covariation and repeatability of male mating effort and mating preferences in a promiscuous fish. Ecology and Evolution, 3: 2020-2029.

Godin, J.-G.J. and Briggs, S.E. 1996. Female mate choice under predation risk in the guppy. Animal Behaviour, 51: 14-130.

Godin, J.-G.J. and Dugatkin, L.A. 1995. Variablility and repeatability of female mating preference in the guppy. Animal Behaviour, 49: 1427-1433.

Godin, J.-G.J. and Dugatkin, L.A. 1996. Female mate preference for bold males in the guppy, Poecilia reticulata. Proceedings of the National Academy of Sciences USA, 93: $10262-10267$.

Godin, J.-G.J., Herdman, E. and Dugatkin, L.A. 2005. Social influences on female mate choice in the guppy, Poecilia reticulata: generalized and repeatable trait-copying behaviour. Animal Behaviour, 69: 999-1005. 
Grether, G.F. 2000. Carotenoid limitation and mate preference evolution: A test of the indicator hypothesis in guppies (Poecilia reticulata). Evolution, 54: 1712-1724.

Grether, G.F., Millie, D.F., Bryant, M.J., Reznick, D.N. and Mayea, W. 2001. Rain forest canopy cover, resource availability, and life history evolution in guppies. Ecology, 82: $1546-1559$.

Grether, G.F., Kolluru, G.R., Rodd, F.H., de la Cerda, J. and Shimazaki, K. 2005. Carotenoid availability affects the development of a colour-based mate preference and the sensory bias to which it is genetically linked. Proceedings of the Royal Society B, 272: $2181-2188$.

Griffiths, S.W. and Magurran, A.E. 1997. Familiarity in schooling fish: how long does it take to acquire? Animal Behaviour, 53: 945-949.

Gross, M.R. 1996. Alternative reproductive strategies and tactics: diversity within sexes. Trends in Ecology and Evolution, 11: 92-98.

Guevara-Fiore, P., Skinner, A. and Watt, P.J. 2009. Do male guppies distinguish virgin females from recently mated ones? Animal Behaviour, 77: 425-431. 
Guevara-Fiore, P., Stapley, J., Krause, J., Ramnarine, I.W. and Watt, P.J. 2010a. Male mate-searching strategies and female cues: how do male guppies find receptive females? Animal Behaviour, 79: 1191-1197.

Guevara-Fiore, P., Stapley, J., and Watt, P.J. 2010b. Mating effort and female receptivity: how do male guppies decide when to invest in sex? Behavioral Ecology and Sociobiology, 64: 1665-1672.

Head, M.L., Wong, B.B.M. and Brooks, R. 2010. Sexual display and mate choice in an energetically costly environment. PLoS ONE, 5: e15279.

Herb, B.M., Biron, S.A. and Kidd, M.R. 2003. Courtship by subordinate male Siamese fighting fish, Betta splendens: their response to eavesdropping and naïve females. Behaviour, 140: 71-78.

Herdman, E.J.E., Kelly, C.D. and Godin, J.-G.J. 2004. Male mate choice in the guppy (Poecilia reticulata): Do males prefer larger females as mates? Ethology, 110: 97-111.

Heuschele, J., Mannerla, M., Gienapp, P. and Candolin, U. 2009. Environment-dependent use of mate choice cues in sticklebacks. Behavioral Ecology, 20: 1223-1227. 
Hibler, T. and Houde, A.E. 2006. The effect of visual obstructions on courtship interference by male guppies: The importance of privacy. Animal Behaviour, 72: 959964.

Hill, G.E. 2006. Female mate choice for ornamental coloration. In: Hill GE and McGraw KJ (eds), Bird Coloration, Volume II. Harvard University Press, Cambridge, MA, pp $137-200$.

Hoefler, C.D., Persons, M.H. and Rypstra, A.L. 2008. Evolutionarily costly courtship displays in a wolf spider: a test of viability indicator theory. Behavioral Ecology and Sociobiology, 19: 974-979.

Holveck, M.-J. and Riebel, K. 2010. Low-quality females prefer low-quality males when choosing a mate. Proceedings of the Royal Society B, 277: 153-60.

Houde, A.E. 1988a. Genetic difference in female choice between two guppy populations. Animal Behaviour, 36: 510-516.

Houde, A.E. 1988b. The effects of female choice and male-male competition on the mating success of male guppies. Animal Behaviour, 36: 888-896.

Houde, A.E. 1992. Sex-linked heritability of a sexually selected character in a natural population of Poecilia reticulata (Pisces: Poeciliidae) (guppies). Heredity, 69: 229-235. 
Houde, A.E. 1994. Effect of artificial selection on male color patterns on mating preference of female guppies. Proceedings of the Royal Society B, 256: 125-130.

Houde, A.E. 1997. Sex, Color, and Mate Choice in Guppies. Princeton University Press, Princeton, NJ.

Houde, A.E. and Endler, J.A. 1990. Correlated evolution of female mating preferences and male color patterns in the guppy Poecilia reticulata. Science, 248: 1405-1408.

Houde, A.E. and Hankes, M.A. 1997. Evolutionary mismatch of mating preferences and male colour patterns in guppies. Animal Behaviour, 53: 343-351.

Houde, A.E. and Torio, A.J. 1992. Effect of parasitic infection on male color pattern and female choice in guppies. Behavioral Ecology, 3: 346-351.

Howard, R.D. 1984. Alternative mating behaviors of young male bullfrogs. American Zoologist, 24: 397-406.

Hoysak, D.J. and Godin, J.-G.J. 2007. Repeatability of male mate choice in the mosquitofish, Gambusia holbrooki. Ethology, 113: 1007-1018. 
Hughes, K.A., Du, L., Rodd, F.H. and Reznick, D.N. 1999. Familiarity leads to female mate preference for novel males in the guppy, Poecilia reticulata. Animal Behaviour, 58: 907-916.

Hunt, J., Brooks, R. and Jennions, M.D. 2005. Female mate choice as a conditiondependent life-history trait. American Naturalist, 166: 79-92.

Jeswiet, S.B. and Godin, J.-G.J. 2011a. Validation of a method for quantifying male mating preferences in the guppy (Poecilia reticulata). Ethology, 117: 422-429.

Jeswiet, S.B., Lee-Jenkins, S.S.Y. and Godin, J.-G.J. 2012. Concurrent effects of sperm competition and female quality on male mate choice in the Trinidadian guppy (Poecilia reticulata). Behavioral Ecology and Sociobiology, 23: 195-200.

Jeswiet, S.B., Lee-Jenkins, S.S.Y., Ramnarine, I.W. and Godin, J.-G.J. 2011 b. Sperm competition risk and mate choice in male Trinidadian guppies, Poecilia reticulata. Animal Behaviour, 81: 639-644.

Ingram, T., Jiang, Y., Rangel, R. and Bolnick, D.I. 2015. Widespread positive but weak assortative mating by diet within stickleback populations. Ecology and Evolution, 5: 3352-3363. 
Jennions, M.D. and Petrie, M. 1997. Variation in mate choice and mating preferences: a review of causes and consequences. Biological Reviews, 72: 283-327.

Jiang, Y., Daniel, I.B. and Kirkpatrick, M. 2013. Assortative mating in animals. American Naturalist, 181: E125-E138.

Jirotkul, M. 1999a. Operational sex ratio influences female preference and male-male competition in guppies. Animal Behaviour, 58: 287-294.

Jirotkul, M. 1999b. Population density influences male-male competition in guppies. Animal Behaviour, 58: 1169-1175.

Jirotkul, M. 2000a. Operational sex ratio influences the opportunity for sexual selection in guppies. Journal of Fish Biology, 56: 739-741.

Jirotkul, M. 2000b. Male trait distribution determined alternative mating tactics in guppies. Journal of Fish Biology, 56: 1427-1434.

Jordan, L.A. and Brooks, R.C. 2010. The lifetime costs of increased male reproductive effort: courtship, copulation and the Coolidge effect. Journal of Evolutionary Biology, 23: $2403-2409$. 
Jordan, L.A. and Brooks, R.C. 2012. Recent social history alters male courtship preferences. Evolution, 66: 280-287.

Karino, K. and Haijima, Y. 2001 Heritability of male secondary sexual traits in feral guppies in Japan. Journal of Ethology, 19: 33-37.

Kelley, J.L., Graves, J.A. and Magurran, A.E. 1999. Familiarity breeds contempt in guppies. Nature, 401: 661-662.

Kelley, K. 2007a. Confidence intervals for standardized effect sizes: Theory, application, and implementation. Journal of Statistical Software, 20: 1-24.

Kelley, K. 2007b. Methods for the behavioral, educational, and social sciences: An R package. Behavior Research Methods, 39: 979-984.

Kelley, K. and Lai, K. 2012. MBESS. R package version 3.3.3, http://CRAN.Rproject.org/package $=$ MBESS

Kelly, C.D, Godin, J.-G.J. and Wright, J.M. 1999. Geographic variation in multiple paternity within natural populations of the guppy (Poecilia reticulata). Proceedings of the Royal Society B, 266: 2403-2408. 
Kendal, R.L., Coolen, I., Van Bergen, Y. and Laland, K.N. 2005. Trade-offs in the adaptive use of social and asocial learning. Advances in the Study of Behavior, 35: 333379.

Kerth, G. and Konig, B. 1999. Fission, fusion and nonrandom associations in female Bechstein's bats (Myotis bechsteinii). Behaviour, 136: 1187-1202.

Kirkpatrick, M. and Dugatkin, L.A. 1994. Sexual selection and the evolutionary effects of copying mate choice. Behavioral Ecology and Sociobiology, 34:443-449.

Kodric-Brown, A. 1993. Female choice of multiple male criteria in guppies: interacting effects of dominance, coloration and courtship. Behavioral Ecology and Sociobiology, 32: 415-420.

Kodric-Brown, A. and Nicoletto, P.F. 2001a. Age and experience affect female choice in the guppy (Poecilia reticulata). American Naturalist, 157: 316-323.

Kodric-Brown, A. and Nicoletto, P.F. 2001b. Female choice in the guppy (Poecilia reticulata): the interaction between male color and display. Behavioral Ecology and Sociobiology, 50: 346-351. 
Kolluru, G.R. and Grether, G.F. 2005. The effects of resource availability on alternate mating tactics in guppies (Poecilia reticulata). Behavioral Ecology and Sociobiology, 16: 294-300.

Kolluru, G.R., Grether, G.F., Dunlop, E. and South, S.H. 2009. Food availability and parasite infection influence mating tactics in guppies (Poecilia reticulata). Behavioral Ecology, 20: 131-137.

Kotrschal, A., Rogell, B., Bundsen, A., Svensson, B., Zajitschek, S., Brännström, I., Immler, S., Maklakov, A.A. and Kolm, N. 2013. Artificial selection on relative brain size in the guppy reveals costs and benefits of evolving a larger brain. Current Biology, 23: $168-171$.

Kurvers, R.H., Van Oers, K., Nolet, B.A., Jonker, R.M., Van Wieren, S.E., Prins, H.H. and Ydenberg, R.C. 2010. Personality predicts the use of social information. Ecology Letters, 13: 829-837.

Krause, J. 1994. The Influence of Food Competition and Prédation Risk on Sizeassortative Shoaling in Juvenile Chub (Leuciscus cephalus). Ethology, 96(2), 105-116.

Lachlan, R.F., Crooks, L. and Laland, K.L. 2008. Who follows whom? Shoaling preferences and social learning learning of foraging information in guppies. Animal Behaviour, 56: 181-190. 
Lehtonen, T.K. and Lindström, K. 2008. Repeatability of mating preferences in the sand goby. Animal Behaviour, 75: 55-61.

Le Roux, A., Cherry, M.I. and Manser, M.B. 2008. The audience effect in a facultatively social mammal, the yellow mongoose, Cynictis penicillata. Animal Behaviour, 75: 943949.

Locatello, L., Rasotto, M.B., Evans, J.P. and Pilastro, A. 2006. Colourful males produce faster and more viable sperm. Journal of Evolutionary Biology, 19: 1595-1602.

Long, K.D. and Houde, A.E. 1989. Orange spots as a visual cue for female mate choice in the guppy (Poecilia reticulata). Ethology, 82: 316-324.

Lusseau, D. 2007. Evidence for social role in a dolphin social network. Evolutionary ecology, 21: 357-366.

Lynch, K.S., Rand, A.S., Ryan, M.J. and Wilczynski, W. 2005. Plasticity in female mate choice associated with changing reproductive states. Animal Behaviour, 69: 689-699.

Lynch, M. and Walsh, B. 1998. Genetics and Analysis of Quantitative Traits. Sinauer Associates, Inc., Sunderland, MA. 
Magellan, K., Pettersson, L.B. and Magurran, A.E. 2005. Quantifying male attractiveness and mating behaviour through phenotypic size manipulation in the Trinidadian guppy, Poecilia reticulata. Behavioral Ecology and Sociobiology, 58: 366-374.

Magurran, A.E. 2005. Evolutionary Ecology: The Trinidadian Guppy. Oxford University Press, Oxford.

Makowicz, A.M., Plath, M. and Schlupp, I. 2010a. Using video playback to study the effect of an audience on male mating behavior in the sailfin molly (Poecilia latipinna). Behavioral Processes, 85: 36-41.

Makowicz, A.M., Plath, M. and Schlupp, I. 2010b. Male guppies (Poecilia reticulata) adjust their mate choice behaviour to the presence of an audience. Behaviour, 147: 16571674.

Mariette, M., Kelley, J.L., Brooks, R. and Evans, J.P. 2006. The effects of inbreeding on male courtship behaviour and coloration in guppies. Ethology, 112: 807-814.

Matos, R.J. and McGregor, P.K. 2002. The effect of the sex of an audience on male-male displays of Siamese fighting fish (Betta splendens). Behaviour, 139: 1211-1221. 
Matos, R. and Schlupp, I. 2005. Performing in front of an audience - signallers and the social environment. In: McGergor, P.K. (ed), Animal Communication Networks. Cambridge University Press, Cambridge, MA, pp 63-83.

Matthews, I.M., Evans, J.P. and Magurran, A.E. 1997. Male display rate reveals ejaculate characteristics in the Trinidadian guppy (Poecilia reticulata). Proceedings of the Royal Society B, 264: 695-700.

Mautz, B.S. and Jennions, M.D. 2011. The effect of competitor presence and relative competitive ability on male mate choice. Behavioral Ecology and Sociobiology, 22: 769775.

McGlothlin, J.W., Moore, A.J., Wolf, J.B., Brodie, E.D. 2010. Interacting phenotypes and the evolutionary process. III. Social evolution. Evolution, 64: 2558-2574.

McGregor, P.K. and Peake, T.M. 2000. Communication networks: social environments for receiving and signalling behaviour. Acta Ethologica, 2: 71-81.

Mery, F., Varela, S.A., Danchin, É., Blanchet, S., Parejo, D., Coolen, I. and Wagner, R.H. 2009. Public versus personal information for mate copying in an invertebrate. Current Biology, 19: 730-734. 
Miller, L.K. and Brooks, R. 2005. The effects of genotype, age, and social environment on male ornamentation, mating behavior, and attractiveness. Evolution, 59: 2414-2425.

Miller, R.G. 1988. Beyond ANOVA: Basics of Applied Statistics. Chapman and Hall CRC Texts in Statistical Science, London.

Moore, A.J., Brodie, E.D. III and Wolf, J.B. 1997. Interacting phenotypes and the evolutionary process: I. Direct and indirect genetic effects of social interactions. Evolution, 51: 1352-1362.

Mourier, J., Vercelloni, J. and Planes, S. 2012. Evidence of social communities in a spatially structured network of a free-ranging shark species. Animal Behaviour, 83: 389401.

Munger, L., Cruz, A. and Applebaum, S. 2004. Mate choice copying in female humpback Limia (Limia nigrofasciata, Family Poeciliidae). Ethology, 110: 563-573.

Nakagawa, S. and Schielzeth, H. 2010. Repeatability for Gaussian and non-Gaussian data: a practical guide for biologists. Biology Reviews, 85: 935-956.

Neff, B.D., Pitcher, T.E. and Ramnarine, I.W. 2008. Inter-population variation in multiple paternity and reproductive skew in the guppy. Molecular Ecology, 17: 29752984. 
Neff, B.D. and Wahl, L.M. 2004. Mechanisms of sperm competition: testing the fair raffle. Evolution, 58: 1846-1851.

Nicoletto, P.F. 1993. Female sexual response to condition-dependent ornaments in the guppy, Poecilia reticulata. Animal Behaviour, 46: 441-450.

Nicoletto, P.F. 1995. Offspring quality and female choice in the guppy, Poecilia reticulata. Animal Behaviour, 49: 377-387.

Nöbel, S. and Witte, K. 2013. Public information influences sperm transfer to females in sailfin molly males. PLoS ONE, 8: e53865.

Nordell, S.E. and Valone, T.J. 1998. Mate choice copying as public information. Ecology Letters, 1: 74-76.

Odell, J.P., Chappell, M.A. and Dickson, K.A. 2003. Morphological and enzymatic correlates of aerobic and burst performance in different populations of Trinidadian guppies, Poecilia reticulata. Journal of Experimental Biology, 206: 3707-3718.

Oh, K.P. and Badyaev, A.V. 2010. Structure of social networks in a passerine bird: Consequences for sexual selection and the evolution of mating strategies. American Naturalist, 176: E80-E89. 
Ojanguren, A.F. and Magurran, A.E. 2004. Uncoupling the links between male mating tactics and female attractiveness. Proceedings of the Royal Society B (Suppl.), 271: S427S429.

Ojanguren, A.F., Evans, J.P. and Magurran, A.E. 2005. Multiple mating influences offspring size in guppies. Journal of Fish Biology, 67: 1184-1188.

Oksanen, J., Blanchet, F.G., Kindt, R., Legendre, P., O'Hara, R.G., Simpson, G.L., Solymos, P., Stevens, M.H.H. and Wagner, H. 2010. vegan: Community Ecology Package. https://cran.r-project.org/web/packages/vegan/index.html

Olsson, M. 1993: Male preference for large females and assortative mating for body size in the sand lizard (Lacerta agilis). Behavioral Ecology and Sociobiology, 32: 337-341.

Ortega, J. and Arita, H.T. 2002. Subordinate males in harem groups of Jamaican fruiteating bats (Artibeus jamaicensis): satellites or sneaks? Ethology, 108: 1077-1091.

Padur, L., Wedekind, J., Öztürk, Ö., Streit, B., Tiedemann, R. and Plath, M. 2009. Do audience effects lead to relaxed male sexual harassment? Behaviour, 146: 1739-1758.

Parker, G.A. 1983. Mate quality and mating decisions. In: Bateson, P. (ed), Mate Choice. Cambridge University Press, Cambridge, UK, pp 141-166. 
Parker, G.A., Ball, M.A., Stockley, P. and Gage, M.J.G. 1996. Sperm competition games: assessment of sperm competition intensity by group spawners. Proceedings of the Royal Society B, 263: 1291-1297.

Parker, G.A., Ball, M.A., Stockley, P. and Gage, M.J. 1997. Sperm competition games: a prospective analysis of risk assessment. Proceedings of the Royal Society B, 264: 17931802.

Patriquin-Meldrum, K.J. and Godin, J.-G.J. 1998. Do female three-spined sticklebacks copy the mate choice of others? American Naturalist, 151: 570-577.

Peake, T.M. 2005. Eavesdropping in communication networks. In: McGregor, P.K. (ed), Animal Communication Networks. Cambridge University Press, Cambridge, UK, pp.1337.

Pettersson, L.B., Ramnarine, I.W., Becher, S.A., Mahabir, R. and Magurran, A.E. 2004. Sex ratio dynamics and fluctuating selection pressures in natural populations of the Trinidadian guppy, Poecilia reticulata. Behavioral Ecology and Sociobiology, 55: 461468.

Piffer, L., Miletto Petrazzini, M.E. and Agrillo, C. 2013. Large number discrimination in newborn fish. PLoS ONE, 8: e62466. 
Pilastro, A. and Bissaza, A. 1999. Insemination efficiency of two alternative male mating tactics in the guppy (Poecilia reticulata). Proceedings of the Royal Society B, 266: 18871891.

Pilastro, A., Evans, J.P., Satorelli, S. and Bisazza, A. 2002. Male phenotype predicts insemination success in guppies. Proceedings of the Royal Society B, 269: 1325-1330.

Pilastro, A., Simonato, M., Bisazza, A. and Evans, J.P. 2004. Cryptic female preference for colorful males in guppies. Evolution, 58: 665-669.

Pilastro, A., Gasparini, C., Boschetto, C. and Evans, J.P. 2008. Colorful male guppies do not provide females with fecundity benefits. Behavioral Ecology, 19: 374-381.

Pitcher, T.E., Neff, B.D., Rodd, F.H. and Rowe, L. 2003. Multiple mating and sequential mate choice in guppies: females trade up. Proceedings of the Royal Society B, 270: 16231629.

Pitcher, T.E., Rodd, F.H. and Rowe, L. 2007. Sexual colouration and sperm traits in guppies. Journal of Fish Biology, 70: 165-177. 
Pitcher, T.E., Rodd, F.H. and Rowe, L. 2008. Female choice and the relatedness of mates in the guppy (Poecilia reticulata): mate choice and inbreeding depression. Genetica, 134: $137-146$.

Pitcher, T.J. and Parrish, J.K. 1993. Functions of shoaling behaviour in teleosts. In: Pitcher, T.J. (ed), Behaviour of Teleost Fishes. Chapman and Hall, London, pp 363-439.

Place, S.S., Todd, P.M., Penke, L. and Asendorpf, J.B. 2010. Humans show mate copying after observing real mate choices. Evolution and Human Behavior, 31: 320-325.

Plath, M. and Bierbach, D. 2011. Sex and the public: Social eavesdropping, sperm competition risk and male mate choice. Communicative and Integrative Biology, 4: 276280.

Plath, M., Blum, D., Schlupp, I. and Tiedemann, R. 2008a. Audience effect alters mating preferences in a livebearing fish, the Atlantic molly, Poecilia mexicana. Animal Behaviour, 75: 21-29.

Plath, M., Blum, D., Tiedemann, R. and Schlupp, I. 2008b. A visual audience effect in a cavefish. Behaviour, 145: 931-947.

Plath, M., Kromuszczynski, K. and Tiedemann, R. 2009. Audience effect alters male but not female mating preferences. Behavioral Ecology and Sociobiology, 63: 381-390. 
Plath, M., Richter, S., Schlupp, I. and Tiedemann, R. 2010. Misleading mollies: surfacebut not cave-dwelling Poecilia mexicana males deceive competitors about mating preferences. Acta Ethology, 13: 49-56.

Plath, M., Richter, S., Tiedemann, R. and Schlupp, I. 2008c. Male fish deceive competitors about mating preferences. Current Biology, 18: 1138-1141.

Plath, M. and Schlupp, I. 2008. Misleading mollies: The effect of an audience on the expression of mating preferences. Communicative and Integrative Biology, 1: 199-203.

Pollick, A.S., Gouzoules, H. and De Waal, F.B.M. 2005. Audience effects on food calls in captive brown capuchin monkeys, Cebus paella. Animal Behaviour, 70: 1273-1281.

Pyron, M. 2003. Female preferences and male-male interactions in zebrafish (Danio rerio). Canadian Journal of Zoology, 81: 122-125.

Qvarnström, A. 2001. Context-dependent genetic benefits from mate choice. Trends in Ecology and Evolution, 16: 5-7.

Reznick, D. and Endler, J.A. 1982. The impact of predation on life history evolution in Trinidadian guppies (Poecilia reticulata). Evolution, 36: 160-177. 
Reznick, D.N., Shaw, F.H., Rodd, F.H. and Shaw, R.G. 1997. Evaluation of the rate of evolution in natural populations of guppies (Poecilia reticulata). Science, 275: 19341937.

Reynolds, J.D., Gross, M.R. 1992. Female mate preference enhances offspring growth and reproduction in a fish, Poecilia reticulata. Proceedings of the Royal Society B. 250: $57-62$.

R Development Core Team. 2012: R: A language and environment for statistical computing, version 2.15.1. R Foundation for Statistical Computing, Vienna, Austria.

R Development Core Team. 2014. R: A Language and Environment for Statistical Computing, version version 3.0.3. R Foundation for Statistical Computing, Vienna, Austria.

Rodd, H.F., Sokolowski, M.B. 1995. Complex origins of variation in the sexual behaviour of male Trinidadian guppies, Poecilia reticulata: interactions between social environment, heredity, body size and age. Animal Behaviour, 49: 1139-1159.

Rodgers, G.M., Ward, J.R., Askwith, B., Morrell, L.J. 2011. Balancing the dilution and oddity effects: decisions depend on body size. PLOS ONE 6: e14819. 
Rosenqvist, G. and Houde, A. 1997: Prior exposure to male phenotypes influences mate choice in the guppy, Poecilia reticulata. Behavioral Ecology, 8: 194-198.

Rowell, J.T. and Servedio, M.R. 2009. Gentlemen prefer blondes: the evolution of mate preference among strategically allocated males. American Naturalist, 173: 12-25.

Striedter, G.F., Freibott, L., Hile, A.G. and Burley, N.T. 2003. For whom the male calls: an effect of audience on contact call rate and repertoire in budgerigars, Melopsittacus undulatus. Animal Behaviour, 65: 875-882.

Rundle, H.D. and Boughman, J.W. 2010. Behavioral ecology and speciation. In: Westneat, D.F. and Fox, C.W. (eds), Evolutionary Behavioral Ecology. Oxford University Press, Oxford, pp 471-487.

Schlupp, I., Marler, C. and Ryan, M.J. 1994. Benefit to male sailfin mollies of mating with heterospecific females. Science, 263: 373-374.

Schlupp, I. and Ryan, M.J. 1997. Male sailfin mollies (Poecilia latipinna) copy the mate choice of other males. Behavioral Ecology and Sociobiology, 8: 104-107.

Schwartz, A.K. and Hendry, A.P. 2007. A test for the parallel co-evolution of male colour and female preference in Trinidadian guppies (Poecilia reticulata). Evolutionary Ecology Research, 9: 71-90. 
Servedio, M.R. and Lande, R. 2006. Population genetic models of male and mutual mate choice. Evolution, 60: 674-685.

Sheridan, L. and Pomiankowski, A. 1997. Female choice for spot asymmetry in the Trinidadian guppy. Animal Behaviour, 54: 1523-1530.

Shohet, A.J. and Watt, P.J. 2004. Female association preference based on olfactory cues in the guppy, Poecilia reticulata. Behavioral Ecology and Sociobiology. 55: 363-369.

Skinner, A.M.J. and Watt, P.J. 2007. Phenotypic correlates of spermatozoon quality in the guppy, Poecilia reticulata. Behavioral Ecology and Sociobiology, 18: 47-52.

Signorell, A. 2015. DescTools: Tools for descriptive statistics. R package version 0.99.15.

Snekser, J.L., McRobert, S.P. and Clotfelter, E.D. 2006. Social partner preferences of male and female fighting fish (Betta splendens). Behavioral Processes, 72: 381-41.

South, S.H., Arnqvist, G. and Servedio, M.R. 2012. Female preference for male courtship effort can drive the evolution of male mate choice. Evolution, 66: 3722-3735.

Stearns, S.C. 1992. The Evolution of Life Histories. Oxford University Press, Oxford. 
Sundaresan, S.R., Fischhoff, I.R., Dushoff, J. and Rubenstein, D.I. 2007. Network metrics reveal differences in social organization between two fission-fusion species, Grevy's zebra and onager. Oecologia, 151: 140-149.

Svensson, O., Nyman, A. and Kvarnemo, C. 2004. Costly courtship or dishonest display? Intensely displaying sand goby males have lower lipid content. Journal of Fish Biology, 64: $1425-1429$.

Syriatowicz, A. and Brooks, R. 2004. Sexual responsiveness is condition-dependent in female guppies, but preference functions are not. BMC Ecology, 4: 5.

Teyssier, A., Bestion, E., Richard, M. and Cote, J. 2014. Partners' personality types and mate preferences: predation risk matters. Behavioral Ecology, 25: 723-733.

Vakirtzis, A. 2011. Mate choice copying and nonindependent mate choice: a critical review. Annales Zoologici Fennici, 48: 91-107.

Valone, T.J. 2007. From eavesdropping on performance to copying the behavior of others: a review of public information use. Behavioral Ecology and Sociobiology, 62: 114. 
Vukomanovic, J. and Rodd, F.H. 2007. Size-dependent female mate copying in the guppy (Poecilia reticulata): large females are role models but small ones are not. Ethology, 113: $579-586$.

Wade, M.J. and Pruett-Jones, S.G. 1990. Female copying increases the variance in male mating success. Proceedings of the National Academy of Sciences, 87: 5749-5753.

Walling, C.A., Royle, N.J., Lindström, J. and Metcalfe, N.B. 2010. Do female association preferences predict the likelihood of reproduction? Behavioral Ecology and Sociobiology, 64: $541-548$.

Ward, A.J.W. and Hart, P.J. 2003. The effects of kin and familiarity on interactions between fish. Fish and Fisheries, 4: 348-358.

Ward, A.J.W., Krause, J. 2001. Body length assortative shoaling in the European minnow, Phoxinus phoxinus. Animal Behaviour, 62: 617-621.

Watt, P.J., Shohet, A.J. and Renshaw, K. 2001. Female choice for good genes and sexbiased broods in guppies. Journal of Fish Biology, 59: 843-850.

Westneat, D., Walters, A., McCarthy, T., Hatch, M. and Hein, W. 2000. Alternative mechanisms of nonindependent mate choice. Animal Behaviour, 59: 467-476. 
Wedell, N., Gage, M.J.G. and Parker, G.A. 2002. Sperm competition, male prudence and sperm-limited females. Trends in Ecology and Evolution, 17: 313-320.

Widemo, M.S. 2005. Male but not female pipefish copy mate choice. Behavioral Ecology and Sociobiology, 17: 255-259.

Widemo, F. and Sæther, S.A. 1999. Beauty is in the eye of the beholder: causes and consequences of variation in mating preferences. Trends in Ecology and Evolution, 14: 26-31.

Witte, K. 2006a. Learning and mate choice. In: Brown, C., Laland, K., Krause, J. (eds), Fish Cognition and Behaviour. Blackwell Publishing Ltd, Oxford, pp 70-95.

Witte, K. 2006b. Time spent with a male is a good indicator of mate preference in female zebra finches. Ethology, Ecology and Evolution, 18: 195-204.

Witte, K., Kniel, N. and Kureck, I M. 2015. Mate-choice copying: Status quo and where to go. Current Zoology, 61: 1073-1081.

Witte, K. and Ryan, M.J. 2002. Mate choice copying in the sailfin molly, Poecilia latipinna, in the wild. Animal Behaviour, 63: 943-949. 
Witte, K. and Ryan, M.J. 1998. Male body length influences mate-choice copying in the sailfin molly Poecilia latipinna. Behavioral Ecology and Sociobiology, 9: 534-539.

Witte, K., Kniel, N. and Kureck, I.M. 2015. Mate-choice copying: status quo and where to go. Current Zoology, 61: 1073-1081.

Wolf, J.B., Brodie, E.D. III and Moore, A.J. 1999. Interacting phenotypes and the evolutionary process. II. Selection resulting from social interactions. American Naturalist, 153: 254-266.

Wong, B.B.M. 2004. Male competition is disruptive to courtship in the Pacific blue-eye. Journal of Fish Biology, 65: 333-341.

Yorzinski, J.L. and Platt, M.L. 2010. Same-sex gaze attraction influences mate-choice copying in humans. PLOS ONE, 5: e9115.

Zajitschek, S.R.K., Evans, J.P. and Brooks, R. 2006. Independent effects of familiarity and mating preferences for ornamental traits in mating decisions in guppies. Behavioral Ecology, 17: 911-916.

Ziege, M., Mahlow, K., Hennige-Schulz, C., Kronmarck, C., Tiedemann, R., Streit, B. and Plath, M. 2009. Audience effects in the Atlantic molly (Poecilia mexicana) - prudent 
male mate choice in response to perceived sperm competition risk? Frontiers in Zoology, 6: $17-24$.

Ziege, M., Padur, L., Duwe, V. and Ramm, A. 2008. Audience effect alters mate choice in male Heterophallus milleri (Poeciliidae). Bulletin of Fish Biology, 10: 87-92.

Zimmer, C., Gavalas, A.S., Kunkel, B., Hanisch, J., Martin, S., Biscoff, S., Plath, M. and Bierbach, D. 2013. Mate choice copying in both sexes of the guppy-the role of sperm competition risk and sexual harassment. In: Geldani, L.M., Davin, M.A. (eds), Sexual Selection: Evolutionary Perspectives, Mating Strategies and Long-term Effects of on Genetic Variation. NOVA Science Publishers, Hauppauge, NY, pp 69-92. 
Appendices 


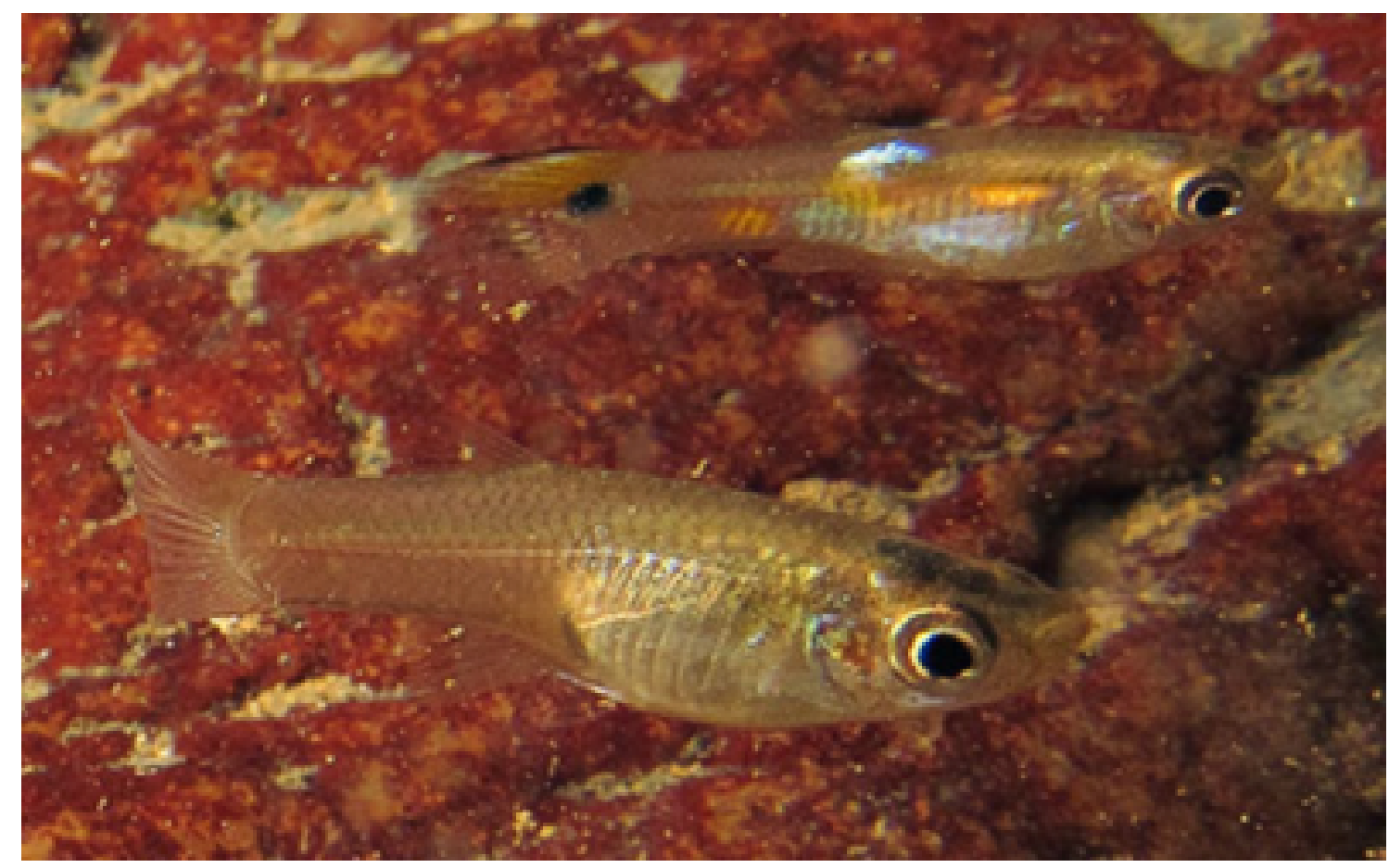

Appendix Figure 1. Photograph of free-ranging adult male (top) and female (bottom) guppies in the Naranjo tributary of the Upper Aripo River, Trinidad. Photo credit: P. Bentzen. 


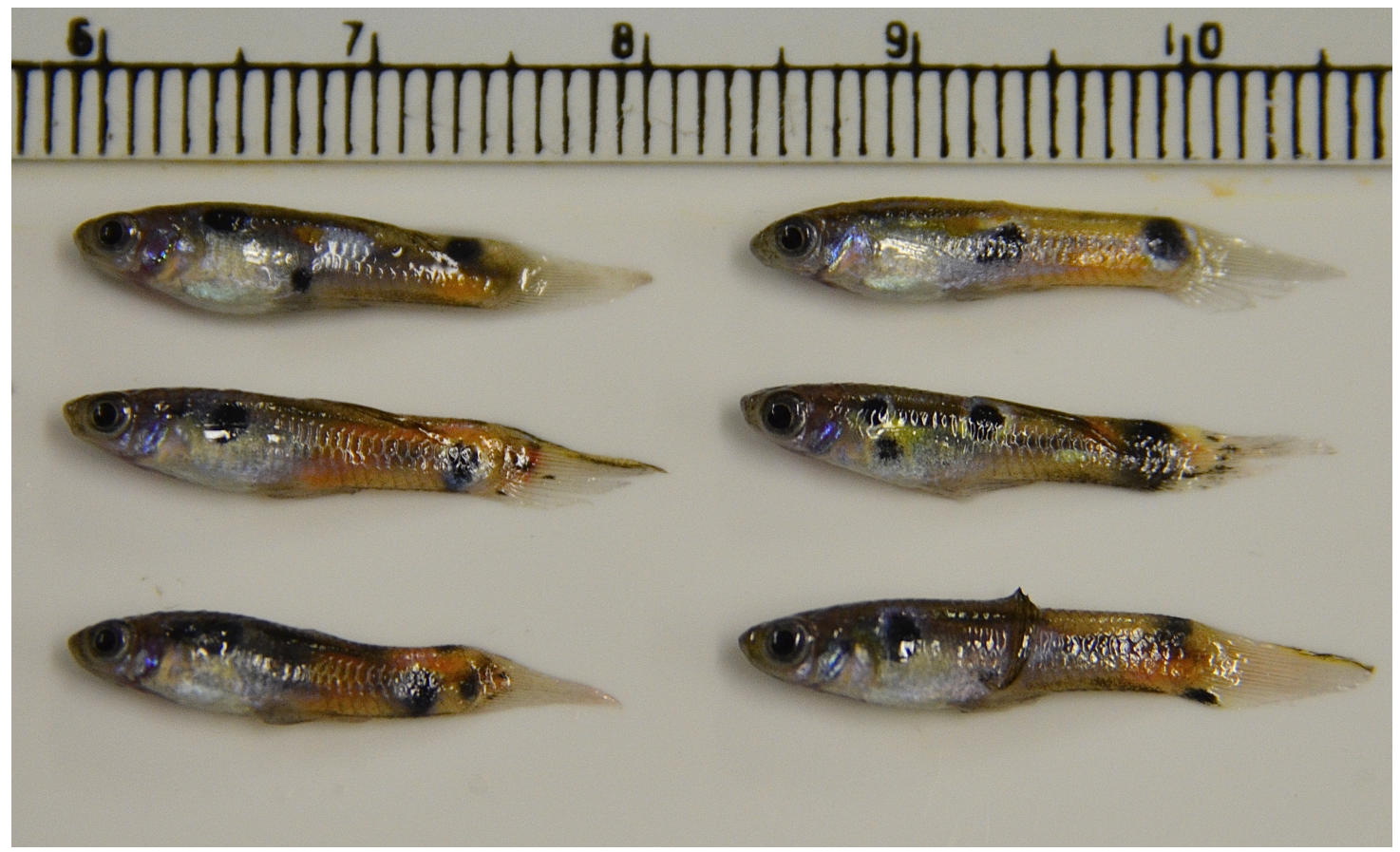

Appendix Figure 2. Examples of individually unique body colour ornamentation pattern in adult male guppies originating from the Upper Aripo River (Naranjo tributary), Trinidad. Photo credit: J.-G.J. Godin. 

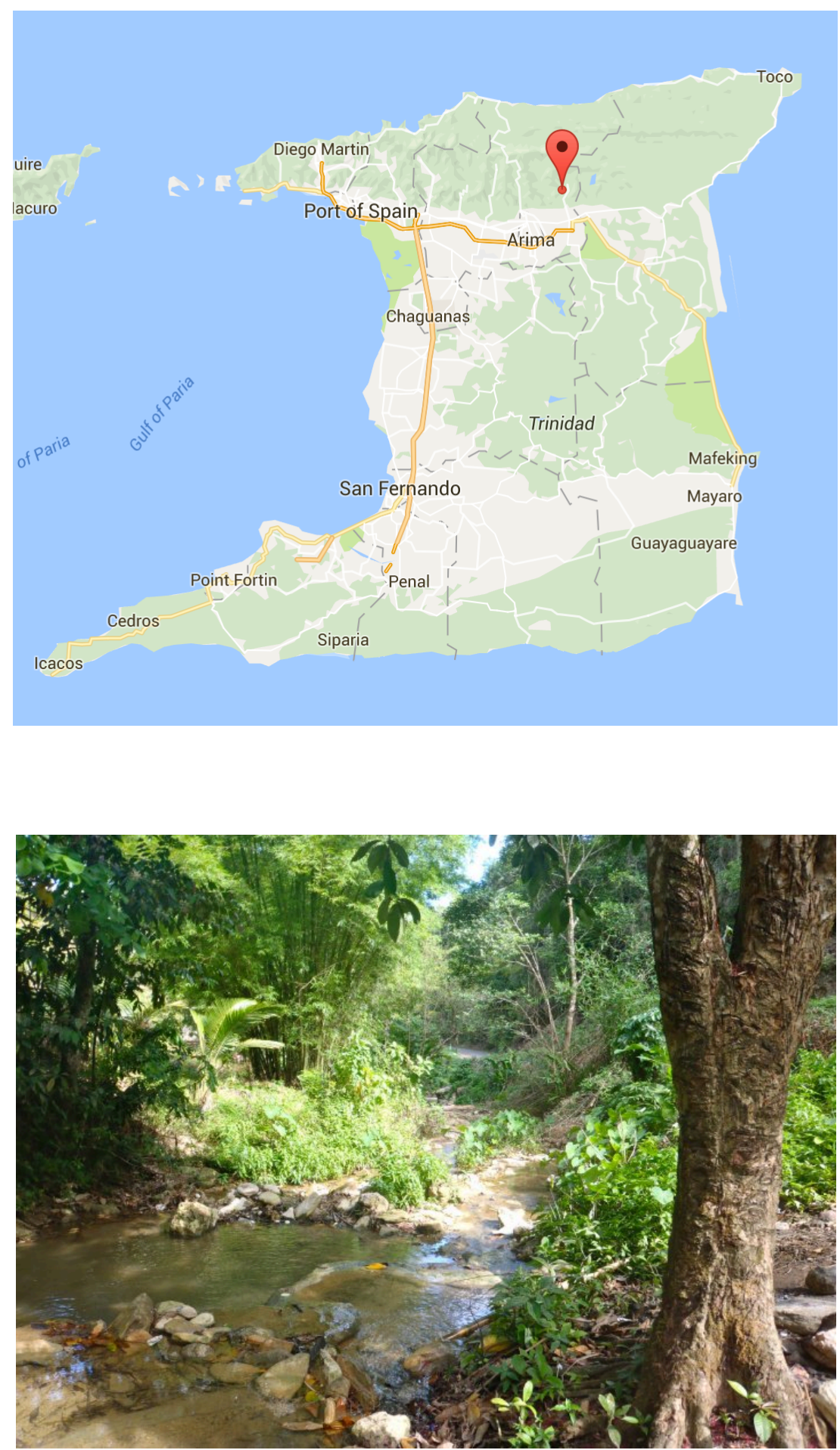

Appendix Figure 3. Google map of Trinidad and a photograph of the Upper Aripo River

(Naranjo Tributary). The red dot in the upper part of the map denotes the general location of the study population. 